Supporting Information

\title{
Regiospecificity in Ligand-Free Pd-Catalyzed C-H Arylation of Indoles: LiHMDS as Base and Transient Directing Group
}

Yorck Mohr, ${ }^{[a]}$ Marc Renom-Carrasco, ${ }^{[b]}$ Clément Demarcy, ${ }^{[b]}$ Elsje Alessandra Quadrelli,,${ }^{[b]}$ Clément Camp, ${ }^{[\mathrm{b}]}$ Florian M. Wisser, ${ }^{[\mathrm{a}]}$ Eric Clot, ${ }^{[\mathrm{c}]}$ Chloé Thieuleux ${ }^{*[\mathrm{~b}]}$ and Jérôme Canivet ${ }^{*[\mathrm{a}]}$ [a]Univ. Lyon, Université Claude Bernard Lyon 1, CNRS, IRCELYON - UMR 5256, 2 Av. Albert Einstein, 69626 Villeurbanne, France

[b]Univ. Lyon, Université Claude Bernard Lyon 1, CPE Lyon, CNRS, C2P2 - UMR 5265, 43 Bvd. du 11 Novembre 1918, 69616 Villeurbanne, France

[c]Institut Charles Gerhardt Montpellier, Université de Montpellier, UMR 5253 CNRS, ENSCM, 8 rue de l'Ecole Normale, Montpellier 34296, France

*jerome.canivet@ircelyon.univ-lyon1.fr *thieuleux@cpe.fr

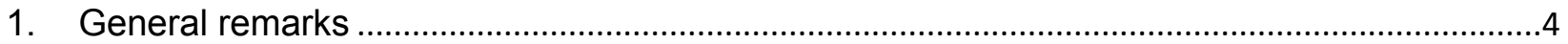

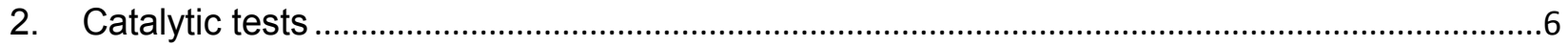

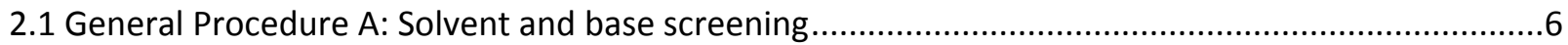

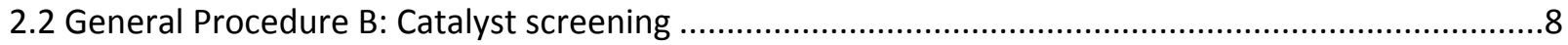

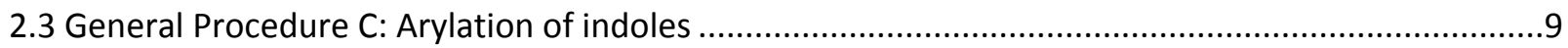

2.4 General Procedure D: Base scope for optimized conditions ........................................................13

2.5 General Procedure E: Solvent \& temperature scope for optimized conditions .............................14

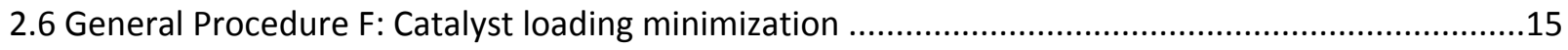

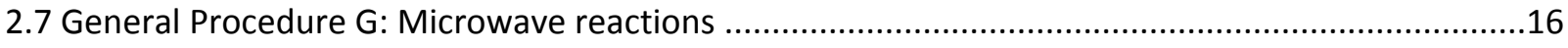

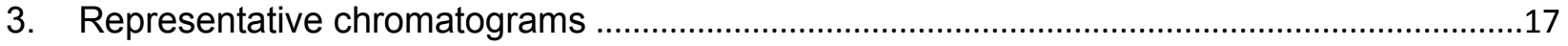

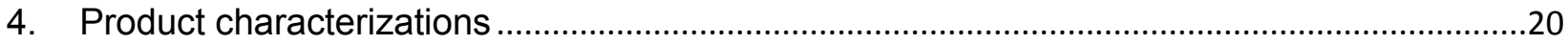

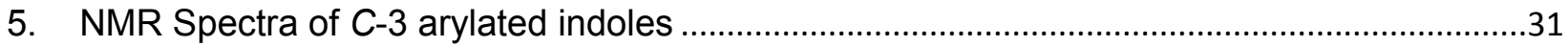

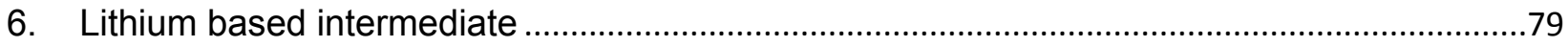

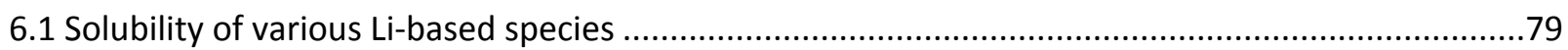




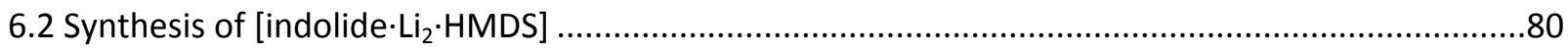

6.3 In-situ formation and solubilization of [indolide $\left.\mathrm{Li}_{2} \cdot \mathrm{HMDS}\right]$......................................................8

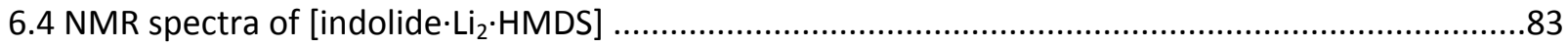

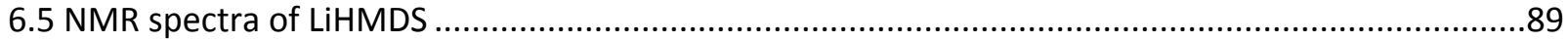

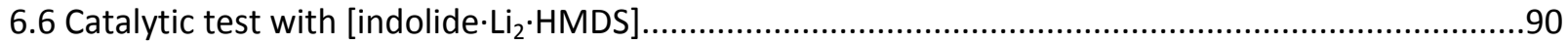

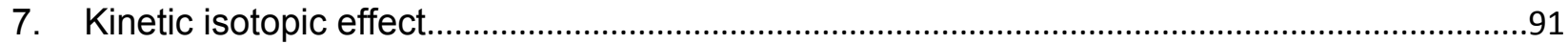

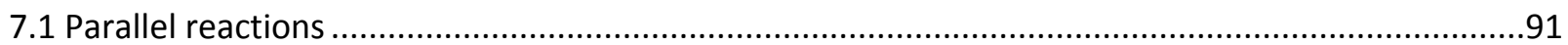

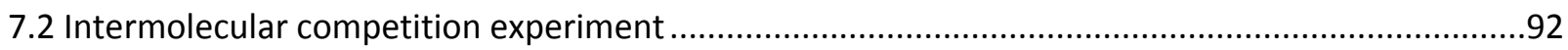

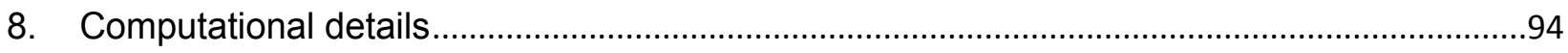

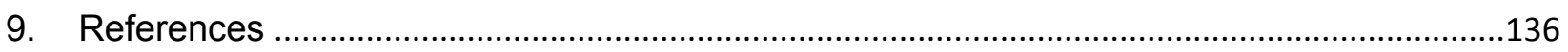




\title{
Abbreviations
}

\author{
Chemicals \& Solvents \\ 2-Me-THF \\ ACN \\ bpy \\ C-2 \\ C-3 \\ DABCO \\ DBU \\ DMAc \\ DMF \\ DMI \\ DMSO \\ KHMDS \\ $\mathrm{KO}{ }^{\mathrm{t}} \mathrm{Bu}$ \\ LDA \\ LiHMDS \\ $\mathrm{LiOAC}$ \\ $\mathrm{LiO}{ }^{t} \mathrm{Bu}$ \\ $\mathrm{Mg}(\mathrm{HMDS})_{2}$ \\ N-1 \\ $\mathrm{N}\left({ }^{(} \mathrm{Pr}\right)_{2} \mathrm{Et}$ \\ NaHMDS \\ $\mathrm{NEt}_{3}$ \\ NMP \\ THF

\section{Other} \\ DFT \\ COSY \\ CPMAS \\ ESI \\ FID \\ GC \\ HMBC \\ HSQC \\ ICP-OES \\ $\mathrm{KIE}$ \\ (HR)MS \\ MAS \\ NMR \\ NOESY \\ 2-methyltetrahydrofuran \\ acetonitrile \\ 2,2'-bipyridine \\ $C-2$ arylation product \\ C-3 arylation product \\ 1,4-diazabicyclo[2.2.2]octane \\ 1,8-diazabicyclo[5.4.0]undec-7-ene \\ $\mathrm{N}, \mathrm{N}$-dimethylacetamide \\ $\mathrm{N}, \mathrm{N}$-dimethylformamide \\ 1,3-dimethyl-2-imidazolidinone \\ dimethyl sulfoxide \\ potassium bis(trimethylsilyl)amide \\ potassium tert-butoxide \\ lithium diisopropylamide \\ lithium bis(trimethylsilyl)amide \\ lithium acetate \\ lithium tert-butoxide \\ magnesium di(bis(trimethylsilyl)amide) \\ $N-1$ arylation product \\ $\mathrm{N}, \mathrm{N}$-diisopropylethylamine \\ sodium bis(trimethylsilyl)amide \\ triethylamine \\ $N$-methyl-2-pyrrolidone \\ tetrahydrofuran \\ density functional theory \\ correlated spectroscopy \\ cross-polarization magic angle spinning \\ electrospray ionization \\ flame ionization detector \\ gas chromatography \\ heteronuclear multiple bond correlation \\ heteronuclear single quantum correlation \\ inductively coupled plasma - optical emission spectroscopy \\ kinetic isotopic effect \\ (high resolution) mass spectrometry \\ magic angle spinning \\ nuclear magnetic resonance \\ nuclear Overhauser effect spectroscopy
}




\section{General remarks}

Liquid nuclear magnetic resonance (NMR) spectra were recorded either on a Bruker Ascend 400 $\left({ }^{1} \mathrm{H}\right.$ at $400.13 \mathrm{MHz},{ }^{13} \mathrm{C}$ at $\left.100.61 \mathrm{MHz}\right)$ or on a Bruker DRX $300\left({ }^{1} \mathrm{H}\right.$ at $300.13 \mathrm{MHz},{ }^{7} \mathrm{Li}$ at 116.64 $\mathrm{MHz},{ }^{13} \mathrm{C}$ at $75.47 \mathrm{MHz}$ ) spectrometer using $\mathrm{CDCl}_{3}, \mathrm{CD}_{2} \mathrm{Cl}_{2}$ or toluene- $d_{8}$ as solvent. Chemical shifts for ${ }^{1} \mathrm{H}$ NMR and for ${ }^{13} \mathrm{C}$ NMR spectra are expressed in ppm relative to the residual solvent signal, ${ }^{7} \mathrm{Li}$ NMR spectra are referenced against $1 \mathrm{M} \mathrm{LiCl}$ as external standard. Data are reported as follows: chemical shift, integration multiplicity $(s=$ singlet, $d=$ doublet, $d d=$ doublet of doublets, $\mathrm{dt}=$ doublet of triplets, $\mathrm{t}=$ triplet, $\mathrm{q}=$ quartet, $\mathrm{m}=$ multiplet, $\mathrm{br}=$ broad signal) and coupling constant ( $\mathrm{J}$ in $\mathrm{Hz}$ ). Solid-state NMR spectra at room temperature were recorded on a Bruker AVANCE III 500 WB spectrometer $\left(11.7 \mathrm{~T},{ }^{1} \mathrm{H}\right.$ at $500.04 \mathrm{MHz},{ }^{7} \mathrm{Li}$ at $194.33 \mathrm{MHz}$ and ${ }^{13} \mathrm{C}$ at $125.73 \mathrm{MHz}$ ) equipped with a $2.5 \mathrm{~mm}$ MAS (magic angle spinning) probe. ${ }^{13} \mathrm{C}$ cross polarization (CP) MAS spectra were referenced against glycine, ${ }^{7} \mathrm{Li}$ direct excitation spectra were referenced against $1 \mathrm{M}$ LiCl solution. Gas Chromatography (GC) FID analysis was conducted on an Agilent Technologies $7890 \mathrm{~A}$ instrument equipped with a HP-5 column (30 $\mathrm{m} \times 0.320 \mathrm{~mm} \times 0.25 \mu \mathrm{m}$, Agilent). GC-MS analysis was conducted on a Shimadzu GCMS-QP-2010 instrument equipped with a HP-5 column (30 $\mathrm{m} \times 0.320 \mathrm{~mm} \times 0.25 \mu \mathrm{m}$, Agilent). High Resolution Mass Spectra (HRMS) were recorded on a Bruker QTOF Impact II using electrospray ionization or an Agilent 7200 GC/Q-TOF using electronic ionization (5 ppm error, Centre Commun de Spectrométrie de Masse, Université Lyon 1). Elemental analyses were performed under inert atmosphere at the Mikroanalytisches Labor Pascher (Remagen, Germany), ICP-OES analysis were done using an Activa from Jobin Yvon. Liquid size-exclusion chromatography was performed using a LC-20AR Semi-Preparative Recycle System (Manual Injector) (Shimadzu) with two size-exclusion PLGel columns (10 $\mu \mathrm{m}, 50 \AA$, Agilent) in series and $\mathrm{CHCl}_{3}$ (Merck, HPLC grade) as eluent.

Unless otherwise stated, all materials were obtained from commercial suppliers and used as received. All reaction solvents were purchased from Sigma Aldrich as anhydrous quality. If this quality was not available reagent grade purity was purchased and the solvent was degassed by three cycles of freeze-pump-thaw and then dried over molecular sieve ( $3 \AA$ ) prior to use. The following compounds were purchased from Sigma Aldrich: 1-iodo-2-methylbenzene (98\%), 1iodo-4-nitrobenzene (98\%), 1-methyl-1H-indole (97\%), 1-iodonaphthalene (97\%), 2,2'-bipyridine (99\%), 2-iodopyridine (98\%), 2-methyl-1H-indole (98\%), 2-phenyl-1H-indole (95\%), 3-iodoaniline (98\%), 3-iodophenol (98\%), 4-bromo-N,N-bis(trimethylsilyl)aniline (97\%), 4-iodophenol (99\%), 4iodo- $\alpha, \alpha, \alpha$-trifluorotoluene (97\%), 5-(trifluoromethyl)- $1 H$-indole (97\%), 5-bromo-1H-indole (99\%), 
5-chloro-1H-indole $\quad(98 \%), \quad 5$-methoxy-1H-indole $\quad(99 \%), \quad 5$-nitro-1H-indole $\quad(98 \%), \quad 6$ bromobenzo[b]thiophene (97\%), $\mathrm{Ag}_{2} \mathrm{CO}_{3}$ (99\%), bis(acetonitrile)dichloropalladium(II) (99.99\%), bis(triphenylphosphine)palladium(II) diacetate (98\%), bromobenzene (99.5\%), bromoferrocene, $\mathrm{CDCl}_{3}$ (99\%), $\mathrm{CD}_{2} \mathrm{Cl}_{2}$ (99\%), chlorobenzene (99.8\%), $\mathrm{Cs}_{2} \mathrm{CO}_{3}$ (99.9\%), Cul (99.5\%), CsOAc (99.99\%), $\mathrm{D}_{2} \mathrm{O}$ (99.99\%), Dabco (99\%), DBU (99\%), DCl (35 wt.\% in $\left.\mathrm{D}_{2} \mathrm{O}\right)$, dodecane (99\%), $1 \mathrm{H}-$ indole $(99 \%)$, iodobenzene (98\%), $\mathrm{K}_{2} \mathrm{CO}_{3}(99 \%)$, KHMDS (95\%), KOtBu (99.99\%), LDA (97\%), $\mathrm{Li}_{2} \mathrm{CO}_{3}(99.997 \%)$, LiHMDS (97\%), LiHMDS (1 M in toluene), LiOAc (99.95\%), LiOtBu (97\%), LProline (99\%), Mg(HMDS) $)_{2}(97 \%), \mathrm{Mg}\left(\mathrm{O}^{t} \mathrm{Bu}\right)_{2}(90 \%), \mathrm{N}\left({ }^{\mathrm{P} r}\right)_{2} \mathrm{Et}(99.5 \%)$, NaHMDS (95\%), $n$-BuLi (2.5 $\mathrm{M}$ in hexane), $\mathrm{NEt}_{3}(99.5 \%)$, palladium(II) acetate (99.9\%), tetrakis(triphenylphosphine)palladium(0) (99\%), THF- $d_{8}(99 \%)$, toluene- $d_{8}(99 \%)$. The following compounds were purchased from TCl Europe: 1-bromo-4-chlorobenzene (99\%), 1-iodo-3-methylbenzene (98\%), 1-iodo-4methoxybenzene (99\%), 1-iodo-4-methylbenzene (99\%), 3-methyl-1H-indole (98\%), 4-bromo$\mathrm{N}, \mathrm{N}$-dimethylaniline (98\%), 5-(4,4,5,5-tetramethyl-1,3,2-dioxaborolan-2-yl)-1H-indole (98\%), 5fluoro- $1 H$-indole (98\%), 5-methyl-1H-indole (99\%), 7-bromo-1H-indole (98\%), 7-methyl- $1 H$-indole (98\%), potassium phenyltrifluoroborate (98\%), p-toluenesulfonyl chloride (99\%).

$1 H$-indole and 2-methyl- $1 H$-indole were sublimed at $10^{-3} \mathrm{mbar}\left(50^{\circ} \mathrm{C}\right)$ prior to use. $\left(2,2^{\prime}-\right.$ Bipyridine)dichloropalladium(II) was synthesized according to literature using bis(acetonitrile)dichloropalladium(II) as Pd precursor. ${ }^{1}$ (2,2'-Bipyridine)palladium(II) diacetate was synthesized according to literature. ${ }^{2}\left(2-{ }^{2} \mathrm{H}\right)-1 H$-indole was synthesized according to literature ${ }^{3}$ using $p$-toluenesulfonyl chloride as sulfonamide precursor and a sublimation as the final purification step $\left(10^{-3} \mathrm{mbar}, 50^{\circ} \mathrm{C}\right)$; $93 \%$ deuterium incorporation (NMR). $\left(3{ }^{2} \mathrm{H}\right)-1 \mathrm{H}$-indole was synthesized according to literature ${ }^{4}$ using a sublimation as the final purification step $\left(10^{-3} \mathrm{mbar}\right.$, $50^{\circ} \mathrm{C}$ ); $96 \%$ deuterium incorporation (NMR). 1-Phenyl-1H-indole was synthesized according to literature. ${ }^{5}$ 7-Phenyl-1H-indole was synthesized according to literature. ${ }^{6}$

Unless otherwise stated, all coupling reactions were prepared in a glove box and conducted under argon atmosphere using $50 \mathrm{~mL}$ glass vessel tubes equipped with a Schlenk tap and heated in a Radleys ${ }^{\circledR}$ Carousel 12 Plus Reaction Station. All work-up and purification procedures were carried out with reagent grade solvents in air. Microwave reactions were conducted using a CEM Discover SP Microwave Synthesizer system $(P=300 \mathrm{~W})$.

Stock solutions of palladium were prepared in flame-dried 8-mL glass vials under argon atmosphere. For stock solution 1 (c $=0.005 \mathrm{~mol} / \mathrm{L}) \mathrm{Pd}(\mathrm{OAc})_{2}(6.74 \mathrm{mg}, 0.03 \mathrm{mmol})$ was dissolved in $6 \mathrm{~mL}$ dry toluene. Stock solution 2 ( $\mathrm{c}=0.00005 \mathrm{~mol} / \mathrm{L}$ ) was prepared by diluting $50 \mu \mathrm{L}$ of stock solution 1 with $5 \mathrm{~mL}$ of dry toluene. 


\section{Catalytic tests}

\subsection{General Procedure A: Solvent and base screening}<smiles>c1ccc2[nH]ccc2c1</smiles>

$0.5 \mathrm{mmol}$<smiles>Ic1ccccc1</smiles>

1.2 equiv.

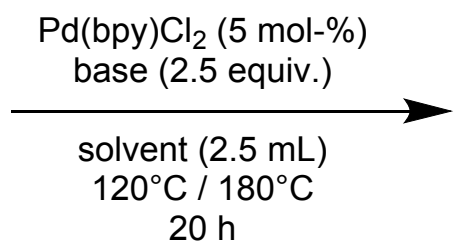

$20 \mathrm{~h}$

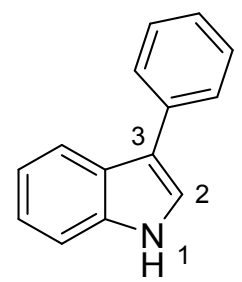

To a flame-dried Schlenk tube equipped with a magnetic stir bar was added indole $(0.5 \mathrm{mmol}$, $59 \mathrm{mg}$ ), base (2.5 equiv.) and (2,2'-bipyridine)dichloropalladium(II) ( $\left.\mathrm{Pd}(\mathrm{bpy}) \mathrm{Cl}_{2}\right)(0.025 \mathrm{mmol}$, $5 \mathrm{~mol}-\%, 8.4 \mathrm{mg}$ ) in a glove box. The flask was sealed with a silicon septum and transferred out of the glove box. Dry solvent $(2.5 \mathrm{~mL})$ was injected through the septum and the mixture was stirred for two minutes. If the base is a liquid it was injected outside of the glovebox through the septum after adding the solvent. Then iodobenzene $(0.6 \mathrm{mmol}, 69 \mu \mathrm{L}, 1.2$ equiv.) was injected through the septum. The reaction was stirred and heated to the desired temperature. For solvents with a boiling point below $120^{\circ} \mathrm{C}$ (toluene, THF, 2-Me-THF, dioxane, monoglyme, ACN), the temperature was set to $120^{\circ} \mathrm{C}$. For solvents with a boiling point equal to or above $120^{\circ} \mathrm{C}$ (mesitylene, diglyme, DMF, NMP, DMI, DMSO) the temperature was set to $180^{\circ} \mathrm{C}$. After $20 \mathrm{~h}$ the reaction mixture was quenched with $1 \mathrm{~mL}$ of deionized water. Then dodecane $(40 \mu \mathrm{L}, 0.174 \mathrm{mmol})$ was added. The aqueous phase was extracted twice with $4 \mathrm{~mL}$ of ethyl acetate, the combined organic phases were washed with $2 \mathrm{~mL}$ of brine and dried over $\mathrm{MgSO}_{4}$. The yield was determined using GC-FID analysis against dodecane as internal standard. 
Table S1: GC-FID yields ${ }^{[a]}$ of 3-phenyl-1H-indole under various conditions following General Procedure A.

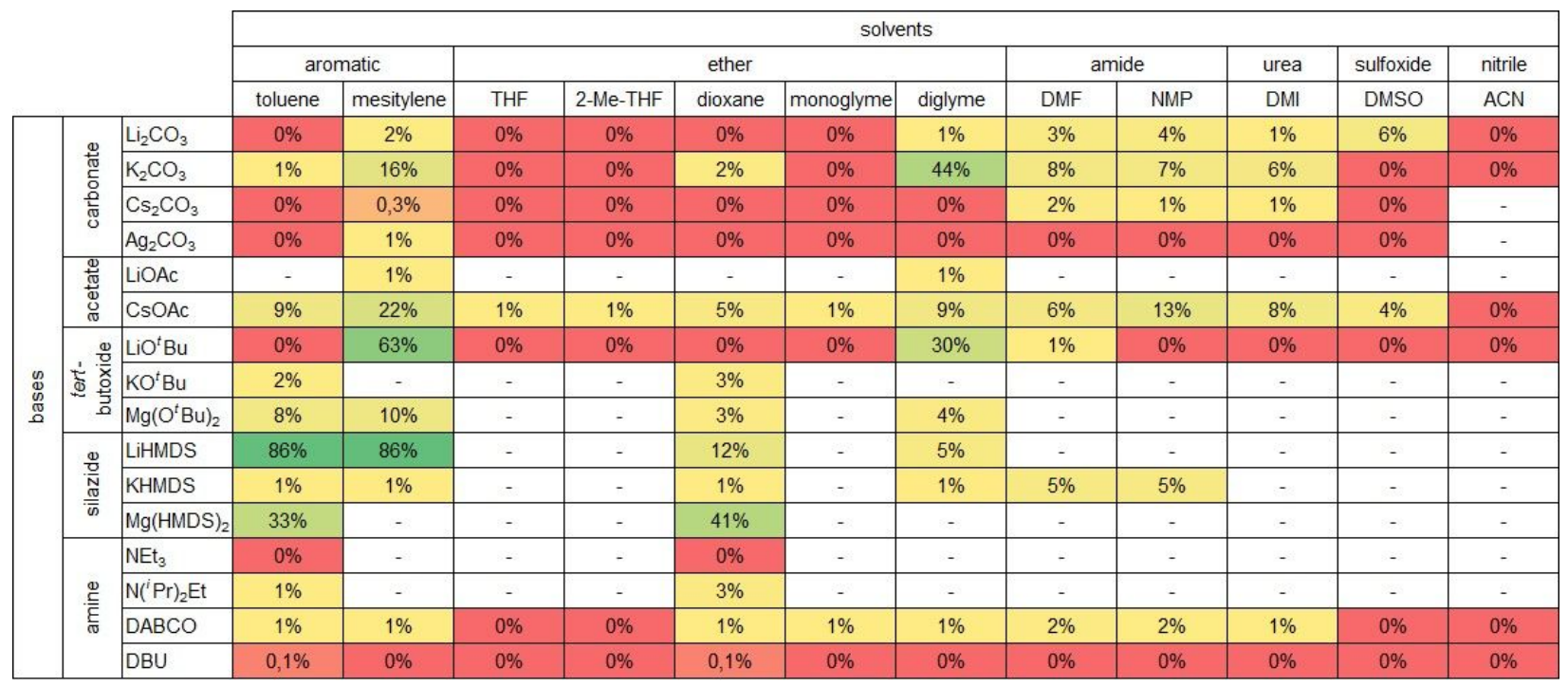

[a] reactions where no yield (-) is indicated have not been conducted.

Table S2: Selectivity[a] for 3-phenyl-1H-indole over N-1 and C-2 arylation products for reactions displayed in Table S1, determined by GC-FID analysis.

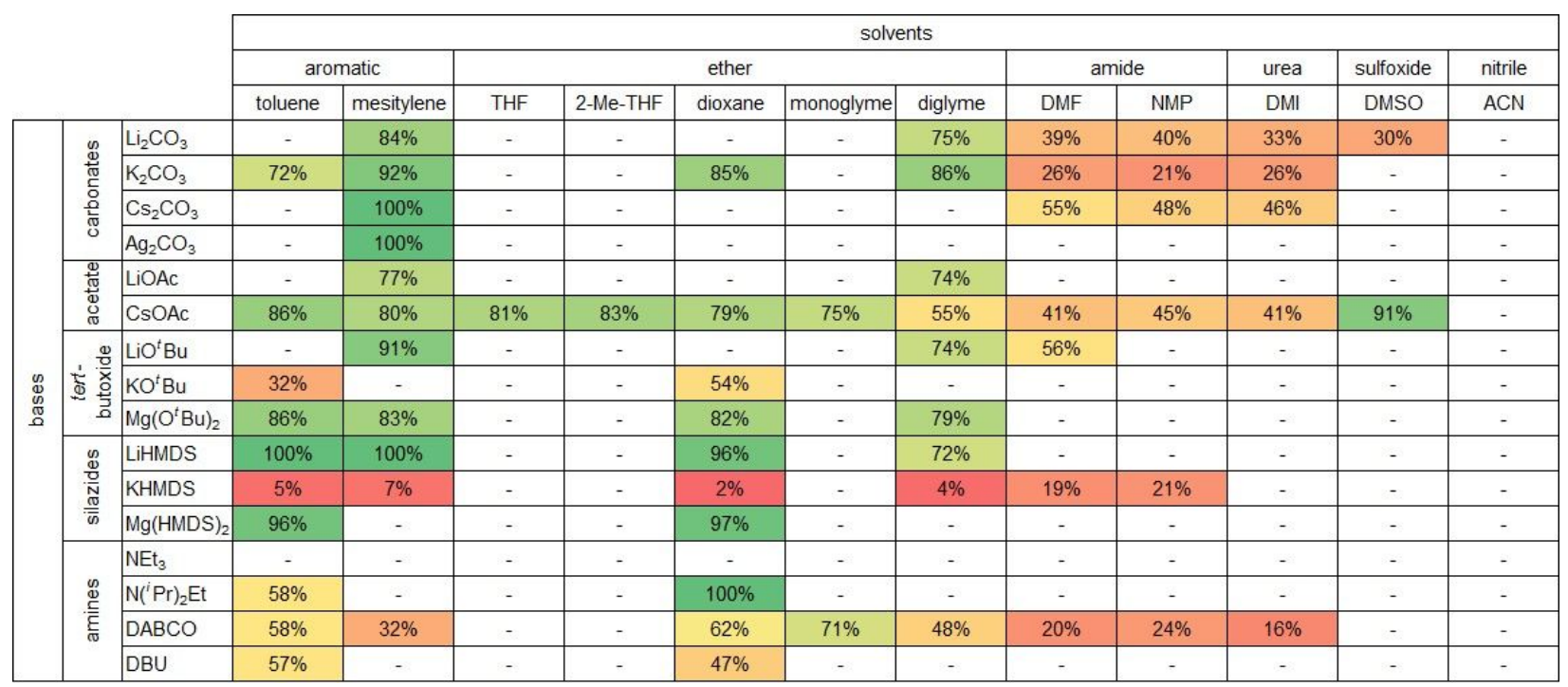

[a] reactions where no selectivity (-) is indicated have either not been conducted or had a GC-FID yield of for 3-phenyl-1H-indole equal to $0 \%$. 


\subsection{General Procedure B: Catalyst screening}

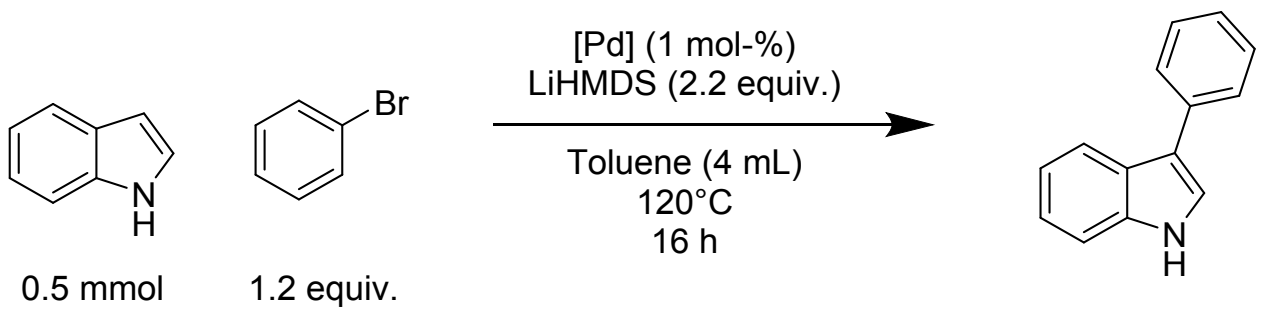

To a flame-dried Schlenk tube equipped with a magnetic stir bar was added indole $(0.5 \mathrm{mmol}$, $59 \mathrm{mg}$ ), LiHMDS (1.1 mmol, $189 \mathrm{mg}, 2.2$ equiv.) and a palladium catalyst in a glove box. The flask was sealed with a silicon septum and transferred out of the glove box. Dry toluene $(4 \mathrm{~mL})$ was injected through the septum and the mixture was stirred for two minutes. Then bromobenzene (0.6 mmol, $64 \mu \mathrm{L}, 1.2$ equiv.) was injected through the septum. The reaction was stirred at $120^{\circ} \mathrm{C}$. After $16 \mathrm{~h}$ the reaction mixture was quenched with $1 \mathrm{~mL}$ of deionized water. Then dodecane (40 $\mu \mathrm{L}, 0.174 \mathrm{mmol}$ ) was added. The aqueous phase was extracted twice with $4 \mathrm{~mL}$ of ethyl acetate, the combined organic phases were washed with $2 \mathrm{~mL}$ of brine and dried over $\mathrm{MgSO}_{4}$. The yield of 3-phenyl-1 $\mathrm{H}$-indole $(C-3)$ was determined using GC-FID analysis against dodecane as internal standard.

Remark: LiHMDS can be indifferently used as solid or as solution (1 $\mathrm{M}$ in toluene).

Table S3: Screening of different palladium sources at 5 and 1 mol-\% catalyst loading following General Procedure B.

\begin{tabular}{llll} 
entry & catalyst $(\mathrm{mol}-\%)$ & $\mathrm{C}-3 \mathrm{GC}$ yield $(\%)$ & comment \\
\hline 1 & $\mathrm{Pd}(\mathrm{bpy}) \mathrm{Cl}_{2}(5)$ & 86 & $\begin{array}{l}1.2 \text { equiv. iodobenzene, } 2.5 \mathrm{~mL} \\
\text { toluene, } 2.5 \text { equiv. LiHMDS, } 20 \mathrm{~h}\end{array}$ \\
2 & $\mathrm{Pd}(\mathrm{bpy}) \mathrm{Cl}_{2}(1)$ & 86 & - \\
3 & $\mathrm{Pd}(\mathrm{bpy})(\mathrm{OAc})_{2}(1)$ & 86 & - \\
4 & $\mathrm{Pd}(\mathrm{OAc})_{2}(1)$ & 87 & - \\
5 & $\mathrm{Pd}\left(\mathrm{PPh}_{3}\right)_{2}(\mathrm{OAc})_{2}(1)$ & 21 & - \\
6 & $\mathrm{Pd}\left(\mathrm{PPh}_{3}\right)_{4}(1)$ & 20 & - \\
7 & - & 0 & no catalyst used
\end{tabular}




\subsection{General Procedure C: Arylation of indoles}

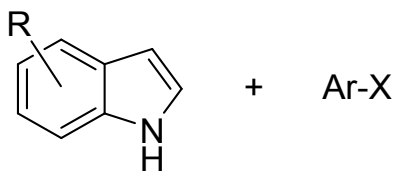

$0.5 \mathrm{mmol}$

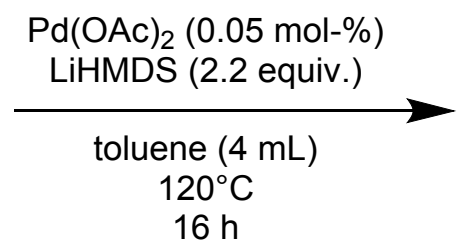

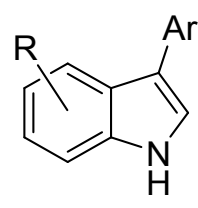

To a flame-dried Schlenk tube equipped with a magnetic stir bar was added indole or an indole derivative $(0.5 \mathrm{mmol})$ and LiHMDS $(1.1 \mathrm{mmol}, 189 \mathrm{mg}, 2.2$ equiv.) in a glove box. The flask was sealed with a silicon septum and transferred out of the glove box. Dry toluene $(4 \mathrm{~mL})$ was injected through the septum and the mixture was stirred for two minutes. Then $50 \mu \mathrm{L}$ of $\mathrm{Pd}(\mathrm{OAc})_{2}$ stock solution $1(0.25 \mu \mathrm{mol}, 0.05 \mathrm{~mol}-\%)$ were injected through the septum along with the corresponding aryl halide ( $0.6 \mathrm{mmol}, 1.2$ equiv.). If the aryl halide is a solid, it was added alongside indole and LiHMDS in the glovebox. The reaction was stirred at $120^{\circ} \mathrm{C}$. After $16 \mathrm{~h}$ the reaction mixture was quenched with $1 \mathrm{~mL}$ of deionized water. Then dodecane $(40 \mu \mathrm{L}, 0.174 \mathrm{mmol})$ as an internal standard for GC-FID analysis was added. The aqueous phase was extracted twice with $4 \mathrm{~mL}$ of ethyl acetate, the combined organic phases were washed with $2 \mathrm{~mL}$ of brine and dried over $\mathrm{MgSO}_{4}$. After removal of the solvent by rotary evaporation, unless otherwise stated, the crude product was purified by liquid size exclusion chromatography.

See Section 4 for product characterizations.

Remark: LiHMDS can be indifferently used as solid or as solution (1 M in toluene).

\section{Note:}

- The reaction conditions described in General Procedure C using indole and bromobenzene as reaction partners will be called "optimized conditions" on the following pages.

- For substrates that were obtained with a yield below $50 \%$, General Procedure C was repeated using $500 \mu \mathrm{L}$ of $\mathrm{Pd}(\mathrm{OAc})_{2}$ stock solution $1(2.5 \mu \mathrm{mol}, 0.5 \mathrm{~mol}-\%)$. The total volume of toluene was kept at $4 \mathrm{~mL}$. 
Table S4: Overview of successful reactions between indole and various aryl halides following General Procedure C.

\begin{tabular}{|c|c|c|c|c|c|c|}
\hline product & aryl halide used & $x$ & entry & $\begin{array}{l}\text { yield } \\
(\%)^{[\mathrm{a}]}\end{array}$ & TON[a,b] & $\begin{array}{c}\text { yield } \\
(\%)^{[c]}\end{array}$ \\
\hline 3-phenyl-1H-indole & & $\begin{array}{l}\mathrm{I} \\
\mathrm{Br} \\
\mathrm{Cl}\end{array}$ & $\begin{array}{l}1 \mathrm{a} \\
1 \mathrm{~b} \\
1 \mathrm{c}\end{array}$ & $\begin{array}{l}95 \\
90 \\
19\end{array}$ & $\begin{array}{r}1900 \\
1800 \\
380\end{array}$ & n.c. \\
\hline 3-(p-tolyl)-1H-indole & & I & 2 & 84 & 1680 & n.c. \\
\hline 3-(m-tolyl $)-1 H$-indole & & I & 3 & 85 & 1700 & n.c. \\
\hline 3-(o-tolyl)-1H-indole & & I & 4 & 50 & 1000 & n.c. \\
\hline 3-(4-methoxyphenyl)-1H-indole & & 1 & 5 & 92 & 1840 & n.c. \\
\hline $\begin{array}{l}\text { 4-(1H-indol-3-yl)- } N, N \text { - } \\
\text { dimethylaniline }\end{array}$ & & $\mathrm{Br}$ & 6 & 61 & 1220 & n.c. \\
\hline 3-(4-chlorophenyl)-1H-indole & & $\mathrm{Br}$ & $7^{[\mathrm{d}]}$ & 35 & 700 & 92 \\
\hline $\begin{array}{l}N \text {-(4-(1H-indol-3-yl)phenyl)- } \\
1,1,1 \text {-trimethyl- } N \text { - } \\
\text { (trimethylsilyl)silanamine }\end{array}$ & & $\mathrm{Br}$ & 8 & 40 & 800 & 73 \\
\hline 3-(naphthalen-1-yl)-1H-indole & & 1 & 9 & 89 & 1780 & n.c. \\
\hline $\begin{array}{l}\text { 3-(benzo }[b] \text { thiophen-6-yl)-1H- } \\
\text { indole }\end{array}$ & & $\mathrm{Br}$ & 10 & 26 & 520 & 55 \\
\hline 3-ferrocenyl-1H-indole & & $\mathrm{Br}$ & 11 & $8^{[\mathrm{e}]}$ & 150 & 26 \\
\hline
\end{tabular}

[a] using 0.05 mol-\% Pd-catalyst; [b] Turnover number defined as (moles of $C-3$ arylation product)/(moles of palladium); [c] using 0.5 mol-\% Pd-catalyst; [d] 3 equiv. of aryl halide used; [e] GC-FID yield; n.c. $=$ not conducted. 
Table S5: Overview of successful reactions between indoles and phenyl halides $(\mathrm{Ph}-\mathrm{X})$ following General Procedure C.

\begin{tabular}{|c|c|c|c|c|c|c|}
\hline product & indole derivative used & $x$ & entry & $\begin{array}{l}\text { yield } \\
(\%)^{[a]}\end{array}$ & $\mathrm{TON}^{[\mathrm{a}]}$ & $\begin{array}{l}\text { yield } \\
(\%)^{[c]}\end{array}$ \\
\hline 5-methyl-3-phenyl-1 $\mathrm{H}$-indole & & $\begin{array}{l}\mathrm{I} \\
\mathrm{Br}\end{array}$ & $\begin{array}{l}1 \mathrm{a} \\
1 \mathrm{~b}\end{array}$ & $\begin{array}{l}90 \\
88\end{array}$ & $\begin{array}{l}1800 \\
1760\end{array}$ & $\begin{array}{l}\text { n.c. } \\
\text { n.c. }\end{array}$ \\
\hline 7-methyl-3-phenyl-1 $\mathrm{H}$-indole & & $\begin{array}{l}\mathrm{I} \\
\mathrm{Br}\end{array}$ & $\begin{array}{l}2 a \\
2 b\end{array}$ & $\begin{array}{l}60 \\
23\end{array}$ & $\begin{array}{r}1200 \\
460\end{array}$ & $\begin{array}{r}\text { n.c. } \\
71\end{array}$ \\
\hline 3,7-diphenyl-1H-indole & & $\begin{array}{l}\mathrm{I} \\
\mathrm{Br}\end{array}$ & $\begin{array}{l}3 a \\
3 b\end{array}$ & $\begin{array}{l}72 \\
66\end{array}$ & $\begin{array}{l}1440 \\
1320\end{array}$ & $\begin{array}{l}\text { n.c. } \\
\text { n.c. }\end{array}$ \\
\hline 5-fluoro-3-phenyl- $1 \mathrm{H}$-indole & & $\begin{array}{l}\mathrm{I} \\
\mathrm{Br}\end{array}$ & $\begin{array}{l}4 a \\
4 b\end{array}$ & $\begin{array}{l}56 \\
25\end{array}$ & $\begin{array}{r}1120 \\
500\end{array}$ & $\begin{array}{r}\text { n.c. } \\
50\end{array}$ \\
\hline 5-chloro-3-phenyl-1H-indole & & $\begin{array}{l}\mathrm{I} \\
\mathrm{Br}\end{array}$ & $\begin{array}{l}5 a \\
5 b\end{array}$ & $\begin{array}{l}78 \\
43\end{array}$ & $\begin{array}{r}1560 \\
860\end{array}$ & $\begin{aligned} & \text { n.c. } \\
& 62\end{aligned}$ \\
\hline 5-bromo-3-phenyl-1H-indole & & I & 6 & 44 & 880 & 55 \\
\hline $\begin{array}{l}\text { 5-methoxy-3-phenyl-1H- } \\
\text { indole }\end{array}$ & & $\begin{array}{l}\mathrm{I} \\
\mathrm{Br}\end{array}$ & $\begin{array}{l}7 \mathrm{a} \\
7 \mathrm{~b}\end{array}$ & $\begin{array}{r}38 \\
\left.14^{[\mathrm{d}]}\right]\end{array}$ & $\begin{array}{l}760 \\
280\end{array}$ & $\begin{array}{l}51 \\
46\end{array}$ \\
\hline
\end{tabular}

3-phenyl-5-(4,4,5,5-

tetramethyl-1,3,2-

dioxaborolan-2-yl)-1H-indole

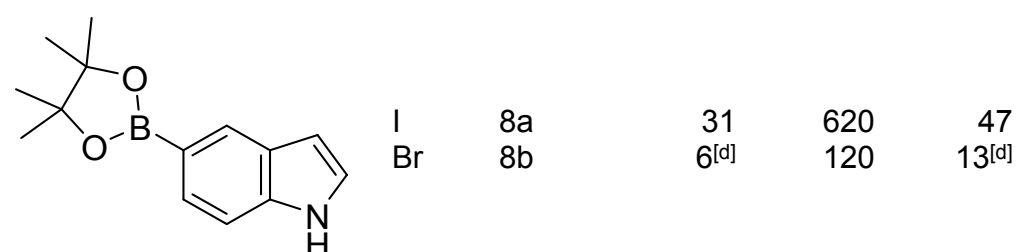

2-methyl-3-phenyl-1H-indole

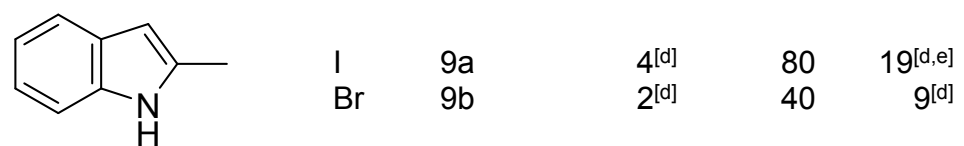

3-phenyl-(2-2H)-1H-indole

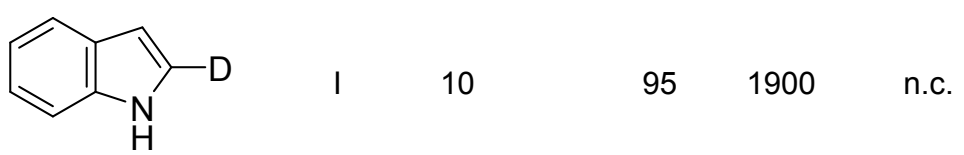

[a] using 0.05 mol-\% Pd-catalyst; [b] Turnover number defined as (moles of C-3 arylation product)/(moles of palladium); [c] using 0.5 mol-\% Pd-catalyst; [d] GC-FID yield; [e] with the use of 2 mol- $\%$ Pd-catalyst the product could be isolated in $80 \%$ yield; n.c. $=$ not conducted. 


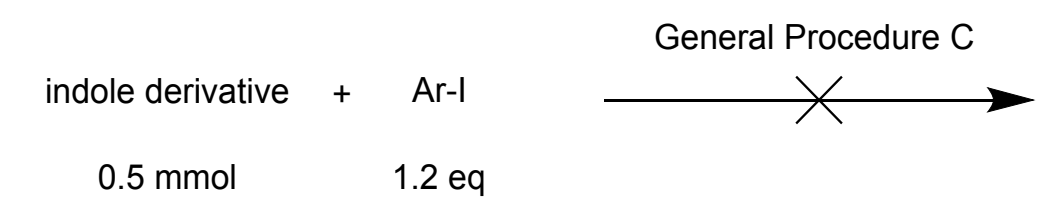

combinations tested

Group 1<smiles>O=[N+]([O-])c1ccc(I)cc1</smiles><smiles>O=[N+]([O-])c1ccc2[nH]ccc2c1</smiles><smiles>FC(F)(F)c1ccc(I)cc1</smiles><smiles>FC(F)(F)c1ccc2[nH]ccc2c1</smiles>

Group 2<smiles>Oc1ccc(I)cc1</smiles><smiles>Oc1cccc(I)c1</smiles><smiles>Nc1cccc(I)c1</smiles><smiles>Cc1c[nH]c2ccccc12</smiles><smiles>Cn1ccc2ccccc21</smiles><smiles>Ic1ccccn1</smiles><smiles>Ic1cc2ccccc2[nH]1</smiles>

Scheme S1: Overview of unsuccessful reactions between indole derivatives and iodoaryls following General Procedure $\mathrm{C}$. For substrates with free hydroxy or amino groups, the reactions were additionally conducted with 4 equiv. of LiHMDS leading to no change in the reaction outcome as compared to when 2.2 equiv. of LiHMDS are used. Group 1: decomposition of the starting materials was observed; Group 2: the starting materials were quantitatively recovered after the reaction. 


\subsection{General Procedure D: Base scope for optimized conditions}<smiles>c1ccc2[nH]ccc2c1</smiles>

$0.5 \mathrm{mmol}$<smiles>Brc1ccccc1</smiles>

1.2 equiv.

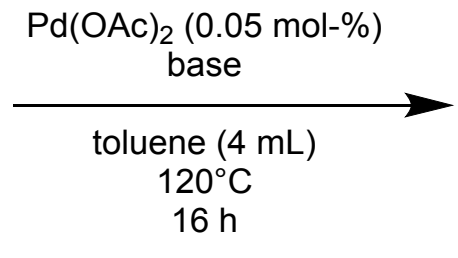

To a flame-dried Schlenk tube equipped with a magnetic stir bar was added indole $(0.5 \mathrm{mmol}$, $59 \mathrm{mg}$ ) and base in a glove box. The flask was sealed with a silicon septum and transferred out of the glove box. In cases where $n$-BuLi was used a solution of that base in hexanes (c = $2.5 \mathrm{~mol} / \mathrm{L}$ ), it was injected outside of the glove box through the septum. Dry toluene $(4 \mathrm{~mL})$ was injected through the septum and the mixture was stirred for two minutes (in the case of combination of bases: 15 minutes). Then $50 \mu \mathrm{L}$ of $\mathrm{Pd}(\mathrm{OAc})_{2}$ stock solution $1(0.25 \mu \mathrm{mol}, 0.05 \mathrm{~mol}-\%)$ were injected through the septum along with bromobenzene $(0.6 \mathrm{mmol}, 64 \mu \mathrm{L}, 1.2$ equiv.). The reaction was stirred at $120^{\circ} \mathrm{C}$. After $16 \mathrm{~h}$ the reaction mixture was quenched with $1 \mathrm{~mL}$ of deionized water. Then dodecane ( $40 \mu \mathrm{L}, 0.174 \mathrm{mmol}$ ) was added. The aqueous phase was extracted twice with 4 $\mathrm{mL}$ of ethyl acetate, the combined organic phases were washed with $2 \mathrm{~mL}$ of brine and dried over $\mathrm{MgSO}_{4}$. The yield of 3-phenyl-1H-indole was determined using GC-FID analysis with dodecane as internal standard.

Table S6: Screening of different bases at a catalyst loading of 0.05 mol-\% $\mathrm{Pd}(\mathrm{OAc})_{2}$ following General Procedure D.

\begin{tabular}{|c|c|c|c|}
\hline entry & base (equiv.) & C-3 GC yield (\%) & comment \\
\hline 1 & LiHMDS (1.0) & 0.6 & - \\
\hline 2 & LiHMDS (1.5) & 38 & - \\
\hline 3 & LiHMDS (2.2) & 90 & - \\
\hline 4 & $\mathrm{LiOtBu}(2.2)$ & 0.1 & - \\
\hline 5 & KO'Bu (2.2) & 1.6 & main product: $N-1(\mathrm{GC}$ yield $=3 \%)$ \\
\hline 6 & NaHMDS (2.2) & 0.4 & main product: $N-1(G C$ yield $=1 \%)$ \\
\hline 7 & KHMDS (2.2) & 0.2 & main product: $N-1($ GC yield $=8 \%)$ \\
\hline 8 & $\mathrm{Mg}(\mathrm{HMDS})_{2}(2.2)$ & 1.5 & - \\
\hline 9 & $\operatorname{LDA}(2.2)$ & 0.3 & main product: $N-1(G C$ yield $=1 \%)$ \\
\hline 10 & BuLi (2.2) & 0 & - \\
\hline 11 & BuLi (0.9) + LiHMDS (1.3) & 84 & - \\
\hline 12 & $\operatorname{BuLi}(0.9)+\mathrm{NaHMDS}(1.3)$ & 0.3 & - \\
\hline 13 & LiHMDS (1.1) + NaHMDS (1.1) & 1.3 & - \\
\hline
\end{tabular}




\subsection{General Procedure E: Solvent \& temperature scope for optimized conditions}

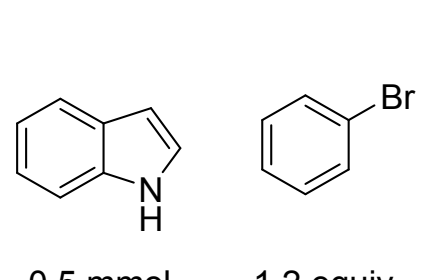

$0.5 \mathrm{mmol}$

1.2 equiv.

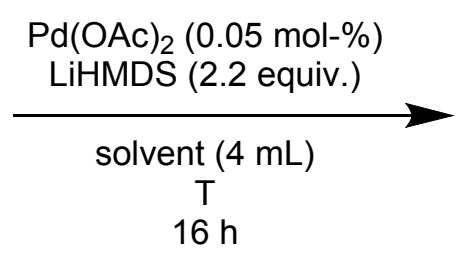

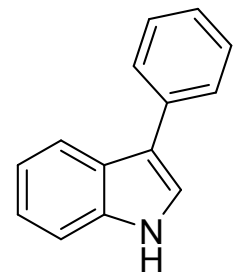

To a flame-dried Schlenk tube equipped with a magnetic stir bar was added indole $(0.5 \mathrm{mmol}$, $59 \mathrm{mg}$ ) and LiHMDS (lithium bis(trimethylsilyl)amide) (1.1 mmol, $189 \mathrm{mg}, 2.2$ equiv.) in a glove box. The flask was sealed with a silicon septum and transferred out of the glove box. Dry solvent $(4 \mathrm{~mL})$ was injected through the septum and the mixture was stirred for two minutes. Then $50 \mu \mathrm{L}$ of $\mathrm{Pd}(\mathrm{OAc})_{2}$ stock solution $1(0.25 \mu \mathrm{mol}, 0.05 \mathrm{~mol}-\%)$ were injected through the septum along with bromobenzene $(0.6 \mathrm{mmol}, 64 \mu \mathrm{L}, 1.2$ equiv.). The reaction was stirred and heated. After $16 \mathrm{~h}$ the reaction mixture was quenched with $1 \mathrm{~mL}$ of deionized water. Then dodecane ( $40 \mu \mathrm{L}, 0.174 \mathrm{mmol}$ ) was added. The aqueous phase was extracted twice with $4 \mathrm{~mL}$ of ethyl acetate and the combined organic phases were washed with $2 \mathrm{~mL}$ of brine and dried over $\mathrm{MgSO}_{4}$. The yield of 3-phenyl-1Hindole was determined using GC-FID analysis against dodecane as internal standard.

Table S7: Screening of different solvents and reaction temperatures at a catalyst loading of $0.05 \mathrm{~mol}-\% \mathrm{Pd}(\mathrm{OAc})_{2}$ following General Procedure E.

\begin{tabular}{llll} 
entry & solvent (temperature) & C-3 GC yield $(\%)$ & comment \\
\hline 1 & toluene $\left(80^{\circ} \mathrm{C}\right)$ & 16 & - \\
2 & toluene $\left(100^{\circ} \mathrm{C}\right)$ & 59 & - \\
3 & toluene $\left(120^{\circ} \mathrm{C}\right)$ & 90 & - \\
4 & toluene $\left(160^{\circ} \mathrm{C}\right)$ & 93 & VWR chemicals, technical $\geq 98 \%$ purity; \\
5 & technical toluene $\left(120^{\circ} \mathrm{C}\right)$ & 89 & contains $\mathrm{H}_{2} \mathrm{O}(\leq 0.05 \%)$ and $\mathrm{O}_{2}(\leq 0.03 \%)^{[a]}$ \\
6 & dioxane $\left(120^{\circ} \mathrm{C}\right)$ & 0 & - \\
7 & THF $\left(80^{\circ} \mathrm{C}\right)$ & 0 & - \\
8 & $2-m e t h y l-\mathrm{THF}\left(80^{\circ} \mathrm{C}\right)$ & 0 & - \\
9 & DMF $\left(120^{\circ} \mathrm{C}\right)$ & 0 & main product: $N-1(\mathrm{GC}$ yield $=4 \%)$ \\
10 & DMAc $\left(120^{\circ} \mathrm{C}\right)$ & 2 & main product: $N-1(\mathrm{GC}$ yield $=16 \%)$ \\
11 & DMSO $\left(120^{\circ} \mathrm{C}\right)$ & 8 & no Pd; main product: $N-1(\mathrm{GC}$ yield $=16 \%)$ \\
12 & DMSO $\left(120^{\circ} \mathrm{C}\right)$ & 8 &
\end{tabular}

[a] for oxygen solubility in toluene see A. Li et al., J. Chem. Eng. Data, 2007, 52, 2339-2344; X. Wu et al., Ind. Eng. Chem. Res. 2014, 53, 9932-9937. 


\subsection{General Procedure F: Catalyst loading minimization}

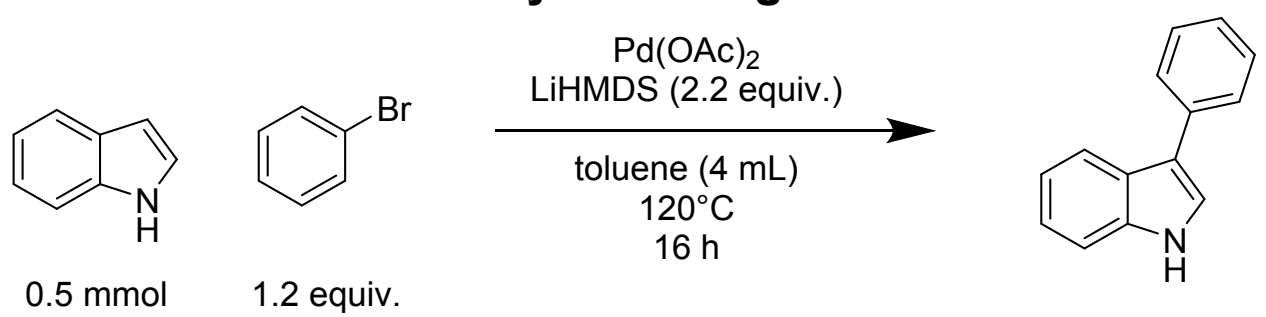

To a flame-dried Schlenk tube equipped with a magnetic stir bar was added indole $(0.5 \mathrm{mmol}$, $59 \mathrm{mg}$ ) and LiHMDS (lithium bis(trimethylsilyl)amide) (1.1 mmol, $189 \mathrm{mg}, 2.2$ equiv.) in a glove box. The flask was sealed with a silicon septum and transferred out of the glove box. Dry toluene $(4 \mathrm{~mL})$ was injected through the septum and the mixture was stirred for two minutes. Then a solution of $\mathrm{Pd}(\mathrm{OAc})_{2}$ in toluene (for $500 \mathrm{ppm}: 50 \mu \mathrm{L}$ of stock solution 1; $100 \mathrm{ppm}: 10 \mu \mathrm{L}$ of stock solution 1; 20 ppm: $200 \mu \mathrm{L}$ of stock solution 2; 5 ppm: $50 \mu \mathrm{L}$ of stock solution 2) was injected through the septum along with bromobenzene $(0.6 \mathrm{mmol}, 64 \mu \mathrm{L}, 1.2$ equiv.). The reaction was stirred at $120^{\circ} \mathrm{C}$. After $16 \mathrm{~h}$ the reaction mixture was quenched with $1 \mathrm{~mL}$ of deionized water. Then dodecane $(40 \mu \mathrm{L}, 0.174 \mathrm{mmol})$ as an internal standard for GC-FID analysis was added. The aqueous phase was extracted twice with $4 \mathrm{~mL}$ of ethyl acetate. The combined organic phases were washed with $2 \mathrm{~mL}$ of brine and dried over $\mathrm{MgSO}_{4}$. After removal of the solvent by rotary evaporation, the crude product was purified by liquid size exclusion chromatography to afford 3phenyl- $1 H$-indole as a white solid.

Remark: LiHMDS can be indifferently used as solid or as solution (1 M in toluene).

Table S8: Screening of different catalyst loadings of $\mathrm{Pd}(\mathrm{OAc})_{2}$ following General Procedure F.

\begin{tabular}{llrr} 
entry & $\mathrm{n}\left(\mathrm{Pd}(\mathrm{OAc})_{2}\right)$ & $C-3$ yield $(\%)$ & TON $^{[\mathrm{a}]}$ \\
\hline 1 & $0.25 \mu \mathrm{mol}(0.05 \mathrm{~mol} \%=500 \mathrm{ppm})$ & 90 & 1800 \\
2 & $0.05 \mu \mathrm{mol}(0.01 \mathrm{~mol}-\%=100 \mathrm{ppm})$ & 46 & 4600 \\
3 & $0.01 \mu \mathrm{mol}(0.002 \mathrm{~mol}-\%=20 \mathrm{ppm})$ & $13^{[b]}$ & 6300 \\
4 & $0.01 \mu \mathrm{mol}(0.002 \mathrm{~mol} \%=20 \mathrm{ppm})^{[\mathrm{cc}]}$ & $13^{[\mathrm{b}]}$ & 6600 \\
5 & $2.5 \mathrm{nmol}(0.0005 \mathrm{~mol}-\%=5 \mathrm{ppm})$ & $2^{[b]}$ & 4200 \\
6 & $2.5 \mathrm{nmol}(0.0005 \mathrm{~mol}-\%=5 \mathrm{ppm})^{[\mathrm{cc}]}$ & $8^{[\mathrm{b}]}$ & 15700 \\
7 & no Pd & 0 & -
\end{tabular}

[a] Turnover number defined as (moles of 3-phenyl-1H-indole)/(moles of palladium); [b] GC-FID yield; [c] reaction performed for 112 hours. 


\subsection{General Procedure G: Microwave reactions}

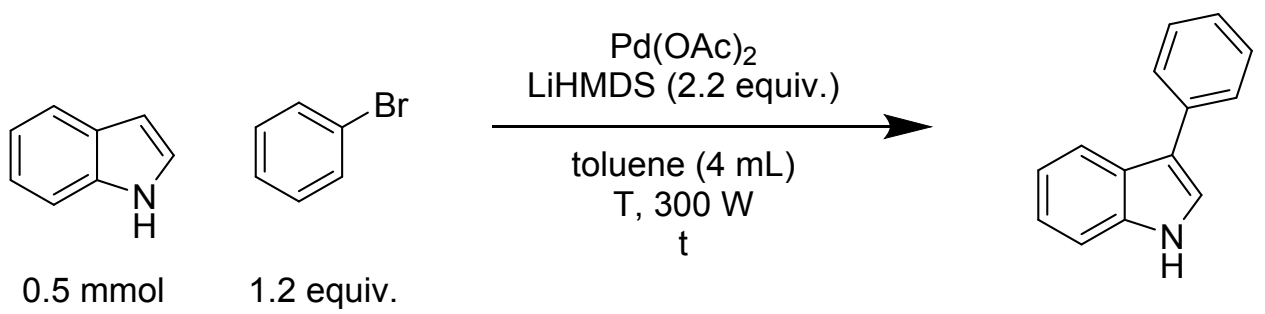

In a glove box, indole $(0.5 \mathrm{mmol}, 59 \mathrm{mg})$ and LiHMDS (lithium bis(trimethylsilyl)amide) $(1.1 \mathrm{mmol}$, $189 \mathrm{mg}, 2.2$ equiv.) were added to a flame-dried $10 \mathrm{~mL}$ microwave glass tube equipped with a magnetic stirring bar. The flask was sealed with a silicon septum and transferred out from the glove box. Dry toluene $(4 \mathrm{~mL})$ was injected through the septum and the mixture was stirred for two minutes. Then, a solution of $\mathrm{Pd}(\mathrm{OAc})_{2}$ in toluene (for $500 \mathrm{ppm}: 50 \mu \mathrm{L}$ of stock solution 1; $20 \mathrm{ppm}$ : $200 \mu \mathrm{L}$ of stock solution 2; $5 \mathrm{ppm}$ : $50 \mu \mathrm{L}$ of stock solution 2) was injected through the septum along with bromobenzene $(0.6 \mathrm{mmol}, 64 \mu \mathrm{L}, 1.2$ equiv.). The septum was exchanged with a pressure control cap. The reaction mixture was further stirred and heated to a given temperature (see Table S9) using microwave irradiation $(P=300 \mathrm{~W})$. After a given time (see Table S9), the reaction mixture was quenched with $1 \mathrm{~mL}$ of deionized water. Then, dodecane $(40 \mu \mathrm{L}, 0.174$ $\mathrm{mmol}$ ) was added as an internal standard for GC-FID analysis. The aqueous phase was extracted twice with $4 \mathrm{~mL}$ of ethyl acetate. The combined organic phases were washed with $2 \mathrm{~mL}$ of brine and dried over $\mathrm{MgSO}_{4}$. After removal of the solvent by rotary evaporation, the crude product was purified by liquid size exclusion chromatography to afford 3-phenyl-1H-indole as a white solid.

Remark: LiHMDS can be indifferently used as solid or as solution (1 M in toluene).

Table S9: Screening of different microwave reaction conditions following General Procedure G.

\begin{tabular}{llrrrrr} 
entry & $\mathrm{n}\left(\mathrm{Pd}(\mathrm{OAc})_{2}\right)(\mathrm{ppm})$ & $\mathrm{T}\left({ }^{\circ} \mathrm{C}\right)$ & $\mathrm{t}(\mathrm{h})$ & $\mathrm{C}-3$ yield $(\%)$ & $\mathrm{TON}^{[\mathrm{a}]}$ & $\mathrm{TOF}^{[\mathrm{b}]}\left(\mathrm{h}^{-1}\right)$ \\
\hline 1 & 500 & 120 & 1 & 29 & 570 & 570 \\
2 & 500 & 120 & 2 & 58 & 1150 & 580 \\
3 & 500 & 150 & 1 & 81 & 1600 & 1600 \\
4 & 20 & 150 & 16 & 23 & 11400 & 710 \\
5 & 20 & 150 & 8 & 15 & 7500 & 940 \\
6 & 5 & 150 & 8 & $6[\mathrm{cc}]$ & 11600 & 1450 \\
7 & no Pd & 150 & 16 & $<1^{[\mathrm{cc}] \mathrm{dd}]}$ & - & -
\end{tabular}

[a] Turnover number defined as (moles of 3-phenylindole)/(moles of palladium) [b] Turnover frequency defined as TON/t; [c] GC-FID yield [d] traces of $N$ arylation product found. 


\section{Representative chromatograms}

All chromatograms shown were recorded using the same temperature program (initial temp. = $55^{\circ} \mathrm{C}$, hold for $1 \mathrm{~min}$; ramp rate $=15^{\circ} \mathrm{C} / \mathrm{min}$ until $250^{\circ} \mathrm{C}$; final temp. $=250^{\circ} \mathrm{C}$ hold for $20 \mathrm{~min}$ ).

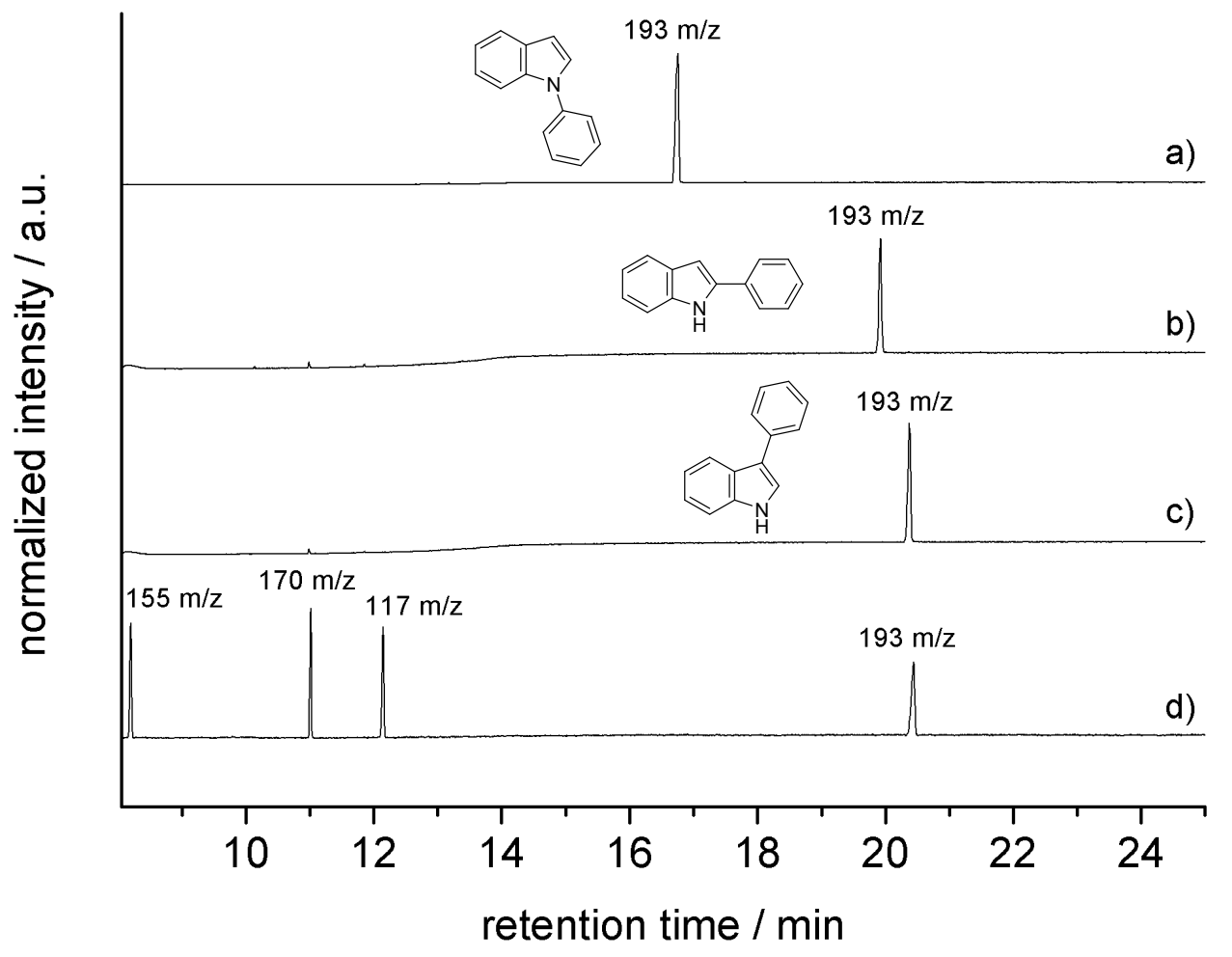

Figure S1: a) GC-MS trace of pure 1-phenyl-1 $H$-indole $\left(193 \mathrm{~m} / \mathrm{z}, t_{\mathrm{R}}=16.7 \mathrm{~min}\right)$; b) GC-MS trace of pure 2-phenyl-1Hindole $\left.\left(193 \mathrm{~m} / \mathrm{z}, t_{R}=19.9 \mathrm{~min}\right) ; \mathbf{c}\right) \mathrm{GC}-\mathrm{MS}$ trace of pure 3-phenyl-1H-indole $\left(193 \mathrm{~m} / \mathrm{z}, t_{\mathrm{R}}=20.4 \mathrm{~min}\right)$; d) GC-MS trace showing the results of a catalytic run (table $S 8$, entry 2 ) with the following signals: $t_{R}=8.2 \mathrm{~min}(155 \mathrm{~m} / \mathrm{z}$, bromobenzene), $t_{R}=11.0 \mathrm{~min}(170 \mathrm{~m} / \mathrm{z}$, dodecane $), t_{R}=12.1 \mathrm{~min}(117 \mathrm{~m} / \mathrm{z}$, indole $), t_{R}=20.4 \mathrm{~min}(193 \mathrm{~m} / \mathrm{z}, 3-$ phenyl-1H-indole $)$. 


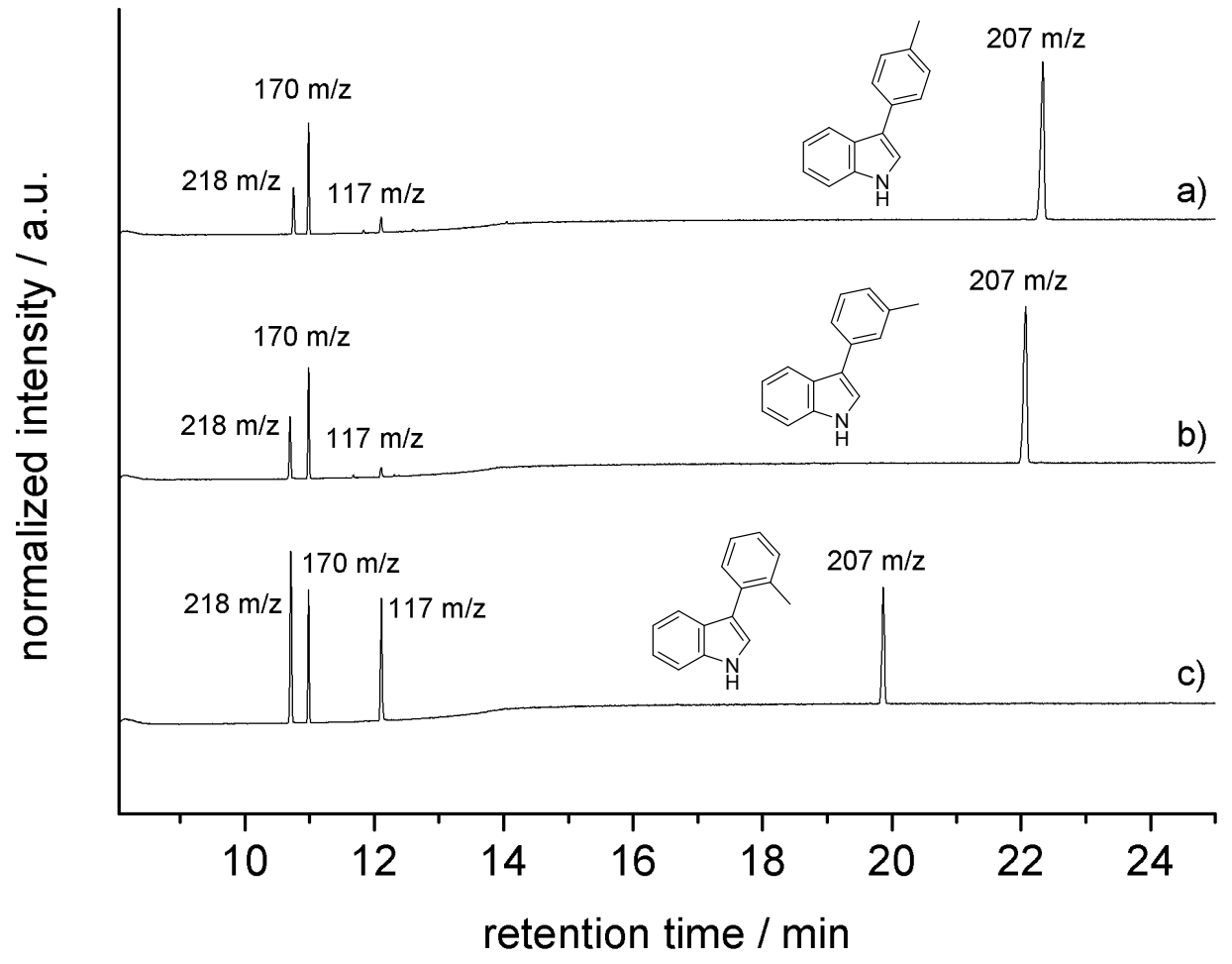

Figure S2: GC-MS traces of the crude reaction mixtures of a) 3-(p-tolyl)-1H-indole (table S4, entry 2$)$ showing signals at $t_{\mathrm{R}}=10.8 \mathrm{~min}(218 \mathrm{~m} / \mathrm{z}, 1$-iodo-4-methylbenzene $), t_{\mathrm{R}}=11.0 \mathrm{~min}(170 \mathrm{~m} / \mathrm{z}$, dodecane $), t_{\mathrm{R}}=12.1 \mathrm{~min}(117 \mathrm{~m} / \mathrm{z}$, indole $)$ and $t_{\mathrm{R}}=22.3 \mathrm{~min}(207 \mathrm{~m} / \mathrm{z}, 3$-(p-tolyl)-1H-indole $) ;$ b) 3 - $(m$-tolyl $)-1 H$-indole (table $\mathrm{S} 4$, entry 3$)$ showing signals at $t_{\mathrm{R}}=$ $10.7 \mathrm{~min}(218 \mathrm{~m} / \mathrm{z}, 1$-iodo-3-methylbenzene $), t_{R}=11.0 \mathrm{~min}(170 \mathrm{~m} / \mathrm{z}$, dodecane $), t_{R}=12.1 \mathrm{~min}(117 \mathrm{~m} / \mathrm{z}$, indole $)$ and $t_{R}$ $=22.1 \mathrm{~min}(207 \mathrm{~m} / \mathrm{z}, 3-(\mathrm{m}$-tolyl)-1H-indole $) ;$ c) 3 -(o-tolyl)- $1 H$-indole (table S4, entry 4$)$ showing signals at $t_{\mathrm{R}}=10.7 \mathrm{~min}$ $\left(218 \mathrm{~m} / \mathrm{z}, 1\right.$-iodo-2-methylbenzene), $t_{\mathrm{R}}=11.0 \mathrm{~min}(170 \mathrm{~m} / \mathrm{z}$, dodecane $), t_{\mathrm{R}}=12.1 \mathrm{~min}(117 \mathrm{~m} / \mathrm{z}$, indole $)$ and $t_{\mathrm{R}}=19.9$ $\min (207 \mathrm{~m} / \mathrm{z}, 3-(o-$ tolyl)- $1 \mathrm{H}$-indole). 


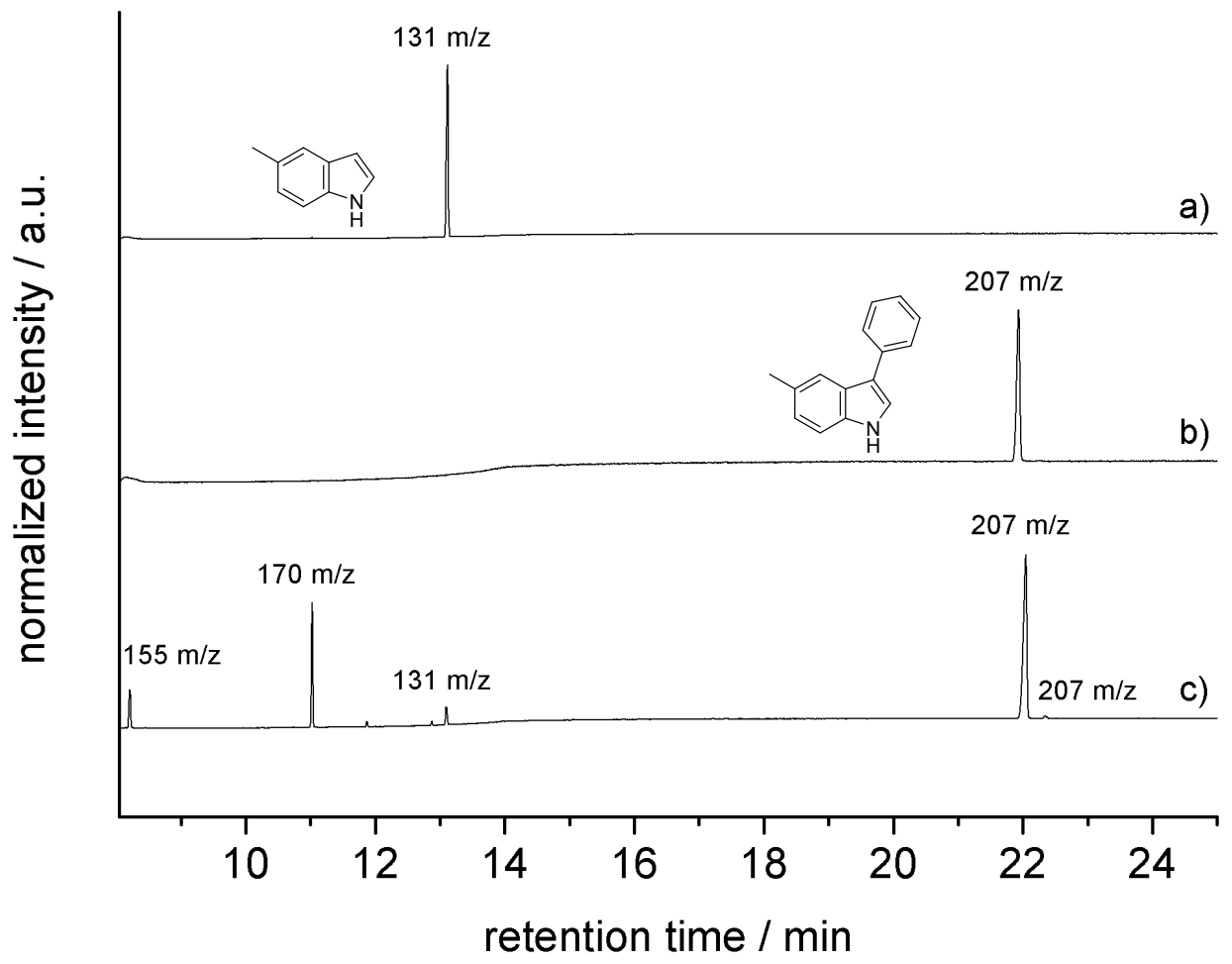

Figure S3: a) GC-MS trace of pure 5-methyl-1H-indole (131 m/z, $\left.t_{\mathrm{R}}=13.1 \mathrm{~min}\right)$; b) GC-MS trace of pure 5-methyl-3phenyl-1 $\mathrm{H}$-indole $\left(207 \mathrm{~m} / \mathrm{z}, t_{\mathrm{R}}=21.9 \mathrm{~min}\right)$; c) GC-MS trace of the crude reaction mixture of 5-methyl-3-phenyl-1H-indole (table S5, entry $1 \mathrm{~b}$ ) showing signals at $t_{\mathrm{R}}=8.2 \mathrm{~min}\left(155 \mathrm{~m} / \mathrm{z}\right.$, bromobenzene), $t_{\mathrm{R}}=11.0 \mathrm{~min}\left(170 \mathrm{~m} / \mathrm{z}\right.$, dodecane), $t_{\mathrm{R}}=$ $13.1 \mathrm{~min}\left(131 \mathrm{~m} / \mathrm{z}, 5\right.$-methyl-1H-indole), $t_{\mathrm{R}}=22.0 \mathrm{~min}(207 \mathrm{~m} / \mathrm{z}, 5$-methyl-3-phenyl-1H-indole $)$. The signal observed at $t_{\mathrm{R}}=22.3 \mathrm{~min}(207 \mathrm{~m} / \mathrm{z})$ might be assigned to traces of 5-methyl-2-phenyl-1H-indole or 5-methyl-1-phenyl- $1 \mathrm{H}$-indole. 


\section{Product characterizations}

The following compounds were synthesized according to General Procedure C (section 2.3).

3-phenyl- $1 H$-indole is obtained as a white solid in $95 \%$ isolated yield (92 $\mathrm{mg}$ ) from iodobenzene, $90 \%$ isolated yield $(87 \mathrm{mg}$ ) from bromobenzene and $19 \%$ isolated yield $(18 \mathrm{mg})$ from chlorobenzene using $0.05 \mathrm{~mol}-\%$ of $\mathrm{Pd}(\mathrm{OAc})_{2}$.

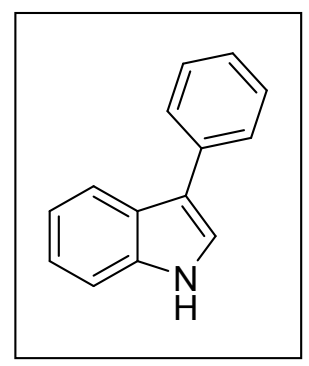

${ }^{1} \mathrm{H}$ NMR $\left(400.13 \mathrm{MHz}, \mathrm{CDCl}_{3}\right) \delta: 8.23(1 \mathrm{H}, \mathrm{br} \mathrm{s}), 7.95(1 \mathrm{H}, \mathrm{d}, J=7.9 \mathrm{~Hz}), 7.68(2 \mathrm{H}, \mathrm{d}, J=7.5 \mathrm{~Hz})$, 7.49 - $7.41(3 \mathrm{H}, \mathrm{m}), 7.38(1 \mathrm{H}, \mathrm{d}, J=2.5 \mathrm{~Hz}), 7.33-7.17(3 \mathrm{H}, \mathrm{m})$.

${ }^{13} \mathrm{C}\left\{{ }^{1} \mathrm{H}\right\}$ NMR $\left(100.61 \mathrm{MHz}, \mathrm{CDCl}_{3}\right) \delta: 136.8,135.7,128.9,127.7,126.1,125.9,122.6,121.9$, $120.5,120.0,118.6,111.5$.

HRMS (ESI): calcd. for $\mathrm{C}_{14} \mathrm{H}_{11} \mathrm{~N}: 192.0813\left(\mathrm{~m} / \mathrm{z}[\mathrm{M}-\mathrm{H}]^{-}\right)$, found $192.0811\left(\mathrm{~m} / \mathrm{z}[\mathrm{M}-\mathrm{H}]^{-}\right)$.

3-phenyl- $\left(2-{ }^{2} \mathrm{H}\right)-1 \mathrm{H}$-indole is obtained as a white solid in $95 \%$ isolated yield $(92 \mathrm{mg})$ and a deuterium incorporation of $93 \%$ (NMR) from iodobenzene and $\left(2-{ }^{2} \mathrm{H}\right)$ - $1 \mathrm{H}$-indole $(93 \%$ initial deuterium incorporation, NMR) using 0.05 mol- $\%$ of $\mathrm{Pd}(\mathrm{OAc})_{2}$.

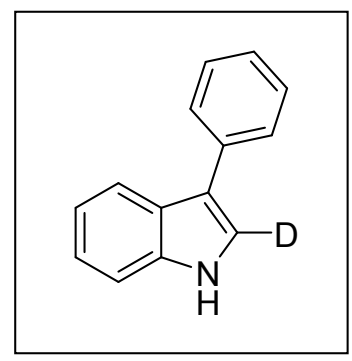

${ }^{1} \mathrm{H}$ NMR $\left(400.13 \mathrm{MHz}, \mathrm{CDCl}_{3}\right) \delta: 8.24(1 \mathrm{H}, \mathrm{br} \mathrm{s}), 7.96(1 \mathrm{H}, \mathrm{d}, J=7.9 \mathrm{~Hz}), 7.68(2 \mathrm{H}, \mathrm{d}, J=7.5 \mathrm{~Hz})$, $7.49-7.41(3 \mathrm{H}, \mathrm{m}), 7.33-7.17(3 \mathrm{H}, \mathrm{m})$.

${ }^{13} \mathrm{C}\left\{{ }^{1} \mathrm{H}\right\}$ NMR $\left(100.61 \mathrm{MHz}, \mathrm{CDCl}_{3}\right) \delta: 136.8,135.7,128.9,127.6,126.1,125.9,122.6,121.7(\mathrm{t}, \mathrm{J}$ $=27.8 \mathrm{~Hz}), 120.5,120.0,118.4,111.5$. 
HRMS (ESI): calcd. for $\left.\mathrm{C}_{14} \mathrm{H}_{10} \mathrm{DN}: 193.0876(\mathrm{~m} / \mathrm{z}[\mathrm{M}-\mathrm{H}]]^{-}\right)$, found $\left.193.0878(\mathrm{~m} / \mathrm{z}[\mathrm{M}-\mathrm{H}]]^{-}\right)$.

3-(p-tolyl)- $1 H$-indole is obtained as an orange solid in $84 \%$ isolated yield $(87 \mathrm{mg})$ from 1 -iodo-4methylbenzene using $0.05 \mathrm{~mol}-\%$ of $\mathrm{Pd}(\mathrm{OAc})_{2}$.

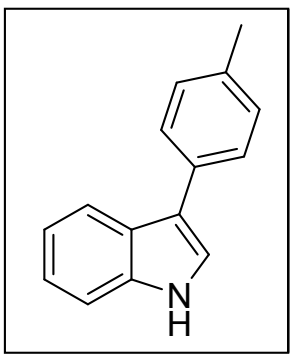

${ }^{1} \mathrm{H}$ NMR $\left(300.13 \mathrm{MHz}, \mathrm{CDCl}_{3}\right) \delta: 8.05(1 \mathrm{H}, \mathrm{br} \mathrm{s}), 7.92(1 \mathrm{H}, \mathrm{d}, J=7.9 \mathrm{~Hz}), 7.55(2 \mathrm{H}, \mathrm{d}, J=8.0 \mathrm{~Hz})$, $7.36(1 \mathrm{H}, \mathrm{d}, J=7.1 \mathrm{~Hz}), 7.28-7.06(5 \mathrm{H}, \mathrm{m}), 2.39(3 \mathrm{H}, \mathrm{s})$.

${ }^{13} \mathrm{C}\left\{{ }^{1} \mathrm{H}\right\}$ NMR $\left(75.47 \mathrm{MHz}, \mathrm{CDCl}_{3}\right) \delta: 136.7,135.7,132.6,129.6,127.5,125.9,122.4,121.6,120.3$, $119.9,118.3,111.5,21.3$.

HRMS (ESI): calcd. for $\mathrm{C}_{15} \mathrm{H}_{14} \mathrm{~N}: 208.1121\left(\mathrm{~m} / \mathrm{z}[\mathrm{M}+\mathrm{H}]^{+}\right)$, found $208.1113\left(\mathrm{~m} / \mathrm{z}[\mathrm{M}+\mathrm{H}]^{+}\right)$.

3-( $m$-tolyl)- $1 H$-indole is obtained as an orange solid in $85 \%$ isolated yield $(88 \mathrm{mg})$ from 1 -iodo-3methylbenzene using $0.05 \mathrm{~mol}-\%$ of $\mathrm{Pd}(\mathrm{OAc})_{2}$.

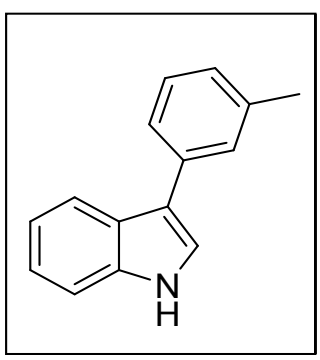

${ }^{1} \mathrm{H}$ NMR $\left(300.13 \mathrm{MHz}, \mathrm{CDCl}_{3}\right)$ ס: $8.11(1 \mathrm{H}, \mathrm{br} \mathrm{s}), 7.94(1 \mathrm{H}, \mathrm{d}, J=7.6 \mathrm{~Hz}), 7.51-7.41(2 \mathrm{H}, \mathrm{m}), 7.41$ - $7.13(5 \mathrm{H}, \mathrm{m}), 7.10(1 \mathrm{H}, \mathrm{d}, J=7.4 \mathrm{~Hz}), 2.41(3 \mathrm{H}, \mathrm{s})$.

${ }^{13} \mathrm{C}\left\{{ }^{1} \mathrm{H}\right\}$ NMR $\left(75.47 \mathrm{MHz}, \mathrm{CDCl}_{3}\right)$ ס: 138.3, 136.6, 135.4, 128.7, 128.3, 126.8, 125.8, 124.6, 122.4 121.8, 120.3, 119.9, 118.4, 111.4, 21.6.

HRMS (ESI): calcd. for $\mathrm{C}_{15} \mathrm{H}_{14} \mathrm{~N}: 208.1121\left(\mathrm{~m} / \mathrm{z}[\mathrm{M}+\mathrm{H}]^{+}\right)$, found $208.1130\left(\mathrm{~m} / \mathrm{z}[\mathrm{M}+\mathrm{H}]^{+}\right)$. 
3-(o-tolyl)- $1 \mathrm{H}$-indole is obtained as an orange solid in $50 \%$ isolated yield (52 mg) from 1-iodo-2methylbenzene using $0.05 \mathrm{~mol}-\%$ of $\mathrm{Pd}(\mathrm{OAc})_{2}$.

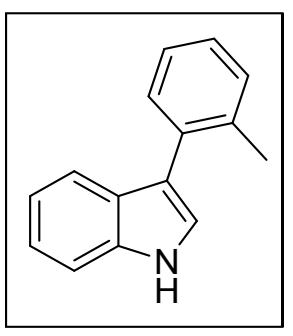

${ }^{1} \mathrm{H}$ NMR $\left(300.13 \mathrm{MHz}, \mathrm{CDCl}_{3}\right)$ ס: $8.15(1 \mathrm{H}, \mathrm{br} \mathrm{s}), 7.52(1 \mathrm{H}, \mathrm{d}, \mathrm{J}=8.1 \mathrm{~Hz}), 7.46-7.34(2 \mathrm{H}, \mathrm{m}), 7.35$ - $7.18(4 \mathrm{H}, \mathrm{m}), 7.18-7.06(2 \mathrm{H}, \mathrm{m}), 2.32(3 \mathrm{H}, \mathrm{s})$.

${ }^{13} \mathrm{C}\left\{{ }^{1} \mathrm{H}\right\}$ NMR $\left(75.47 \mathrm{MHz}, \mathrm{CDCl}_{3}\right) \delta: 137.0,136.0,134.6,131.0,130.5,127.3,126.9,125.8,122.9$, 122.3, 120.3, 120.0, 117.6, 111.3, 20.9.

HRMS (ESI): calcd. for $\mathrm{C}_{15} \mathrm{H}_{14} \mathrm{~N}: 208.1121\left(\mathrm{~m} / \mathrm{z}[\mathrm{M}+\mathrm{H}]^{+}\right)$, found $208.1127\left(\mathrm{~m} / \mathrm{z}[\mathrm{M}+\mathrm{H}]^{+}\right)$.

3-(4-methoxyphenyl)-1H-indole is obtained as an orange solid in $92 \%$ isolated yield $(103 \mathrm{mg})$ from 1-iodo-4-methoxybenzene using 0.05 mol- $\%$ of $\mathrm{Pd}(\mathrm{OAc})_{2}$.

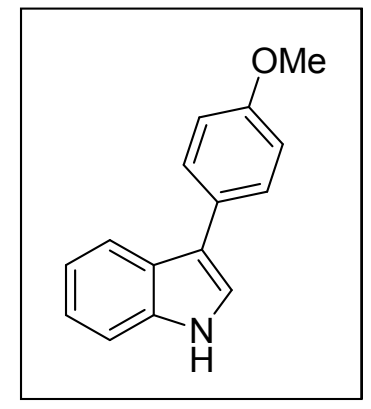

${ }^{1} \mathrm{H}$ NMR $\left(300.13 \mathrm{MHz}, \mathrm{CDCl}_{3}\right) \delta: 8.13(1 \mathrm{H}, \mathrm{br} \mathrm{s}), 7.87(1 \mathrm{H}, \mathrm{d}, J=7.3 \mathrm{~Hz}), 7.56(2 \mathrm{H}, \mathrm{d}, J=8.7 \mathrm{~Hz})$, $7.38(1 \mathrm{H}, \mathrm{d}, J=7.6 \mathrm{~Hz}), 7.28-7.10(3 \mathrm{H}, \mathrm{m}), 6.98(2 \mathrm{H}, \mathrm{d}, J=8.7 \mathrm{~Hz}), 3.83(3 \mathrm{H}, \mathrm{s})$.

${ }^{13} \mathrm{C}\left\{{ }^{1} \mathrm{H}\right\}$ NMR $\left(75.47 \mathrm{MHz}, \mathrm{CDCl}_{3}\right) \delta: 158.2,136.7,128.7,128.2,126.0,122.4,121.3,120.3,119.9$, 118.1, 114.4, 111.5, 55.5.

HRMS (ESI): calcd. for $\mathrm{C}_{15} \mathrm{H}_{14} \mathrm{NO}: 224.1070\left(\mathrm{~m} / \mathrm{z}[\mathrm{M}+\mathrm{H}]^{+}\right)$, found $224.1068\left(\mathrm{~m} / \mathrm{z}[\mathrm{M}+\mathrm{H}]^{+}\right)$.

4-(1H-indol-3-yl)- $N, N$-dimethylaniline was purified by flash column chromatography using a mixture of petroleum ether and ethyl acetate (7:3), plus $0.5 \%$ of $\mathrm{NEt}_{3}$. The product is obtained as 
a colorless oil in $61 \%$ isolated yield $(72 \mathrm{mg}$ ) from 4-bromo- $N, N$-dimethylaniline using 0.05 mol- $\%$ of $\mathrm{Pd}(\mathrm{OAc})_{2}$.

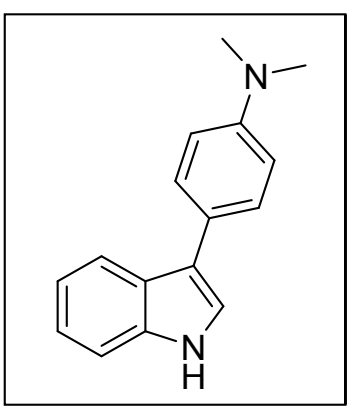

${ }^{1} \mathrm{H}$ NMR $\left(300.13 \mathrm{MHz}, \mathrm{CD}_{2} \mathrm{Cl}_{2}\right)$ ס: $8.30(1 \mathrm{H}, \mathrm{br} \mathrm{s}), 7.94(1 \mathrm{H}, \mathrm{d}, J=7.8 \mathrm{~Hz}), 7.58(2 \mathrm{H}, \mathrm{d}, J=8.6$ $\mathrm{Hz}), 7.42(1 \mathrm{H}, \mathrm{d}, J=7.9 \mathrm{~Hz}), 7.27-7.14(3 \mathrm{H}, \mathrm{m}), 6.88(2 \mathrm{H}, \mathrm{d}, J=8.4 \mathrm{~Hz}), 3.01(6 \mathrm{H}, \mathrm{s})$.

${ }^{13} \mathrm{C}\left\{{ }^{1} \mathrm{H}\right\}$ NMR $\left(75.47 \mathrm{MHz}, \mathrm{CD}_{2} \mathrm{Cl}_{2}\right) \delta: 149.8,137.2,128.6,126.4,124.5,122.5,121.4,120.4$, 120.2, 118.5, 113.7, 111.9, 41.1.

HRMS (ESI): calcd. for $\mathrm{C}_{16} \mathrm{H}_{17} \mathrm{~N}_{2}: 237.1386\left(\mathrm{~m} / \mathrm{z}[\mathrm{M}+\mathrm{H}]^{+}\right)$, found $237.1378\left(\mathrm{~m} / \mathrm{z}[\mathrm{M}+\mathrm{H}]^{+}\right)$.

3-(4-chlorophenyl)-1H-indole was purified by flash column chromatography using a mixture of petroleum ether and ethyl acetate (7:3). The product is obtained as an orange solid in $35 \%$ isolated yield $\left(40 \mathrm{mg}\right.$ ) using $0.05 \mathrm{~mol}-\%$ of $\mathrm{Pd}(\mathrm{OAc})_{2}$ and three equivalents of 1-chloro-4-bromobenzene. With $0.5 \mathrm{~mol}-\%$ of $\mathrm{Pd}(\mathrm{OAc})_{2}$ and three equivalents of 1-chloro-4-bromobenzene, the title compound is obtained in $92 \%$ isolated yield $(105 \mathrm{mg})$.

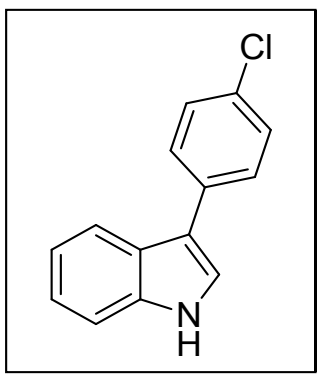

${ }^{1} \mathrm{H}$ NMR $\left(300.13 \mathrm{MHz}, \mathrm{CDCl}_{3}\right) \delta: 8.28(1 \mathrm{H}, \mathrm{br} \mathrm{s}), 7.91(1 \mathrm{H}, \mathrm{d}, J=7.5 \mathrm{~Hz}), 7.62(2 \mathrm{H}, \mathrm{d}, J=8.5 \mathrm{~Hz})$, 7.51 - $7.34(4 \mathrm{H}, \mathrm{m}), 7.35-7.15(2 \mathrm{H}, \mathrm{m})$.

${ }^{13} \mathrm{C}\left\{{ }^{1} \mathrm{H}\right\}$ NMR $\left(75.47 \mathrm{MHz}, \mathrm{CDCl}_{3}\right) \delta: 136.7,134.1,131.7,129.0,128.7,125.6,122.7,122.0$, 120.64, 119.7, 117.3, 111.7.

HRMS (ESI): calcd. for $\mathrm{C}_{14} \mathrm{H}_{11} \mathrm{CIN}: 228.0575\left(\mathrm{~m} / \mathrm{z}[\mathrm{M}+\mathrm{H}]^{+}\right)$, found $228.0572\left(\mathrm{~m} / \mathrm{z}[\mathrm{M}+\mathrm{H}]^{+}\right)$. 
$\mathrm{N}$-(4-(1H-indol-3-yl)phenyl)-1,1,1-trimethyl- $N$-(trimethylsilyl)silanamine is obtained as an orange oil in $40 \%$ isolated yield $(70 \mathrm{mg}$ ) from 4-bromo- $N, N$-bis(trimethylsilyl)aniline using $0.05 \mathrm{~mol}-\%$ of $\mathrm{Pd}(\mathrm{OAc})_{2}$. With $0.5 \mathrm{~mol}-\%$ of $\mathrm{Pd}(\mathrm{OAc})_{2}$, the title compound is obtained in $73 \%$ isolated yield (129 mg).

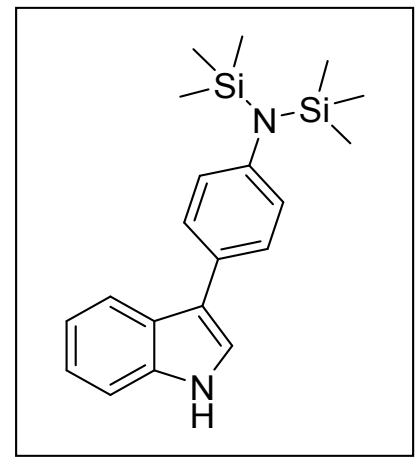

${ }^{1} \mathrm{H}$ NMR $\left(400.13 \mathrm{MHz}, \mathrm{CDCl}_{3}\right)$ ס: $8.17(1 \mathrm{H}, \mathrm{br} \mathrm{s}), 7.95(1 \mathrm{H}, \mathrm{d}, \mathrm{J}=7.8 \mathrm{~Hz}), 7.54-7.48(2 \mathrm{H}, \mathrm{m}), 7.42$ $(1 \mathrm{H}, \mathrm{d}, J=7.9 \mathrm{~Hz}), 7.34(1 \mathrm{H}, \mathrm{d}, J=2.5 \mathrm{~Hz}), 7.27-7.16(2 \mathrm{H}, \mathrm{m}), 6.98-6.93(2 \mathrm{H}, \mathrm{m}), 0.11(18 \mathrm{H}, \mathrm{s})$. ${ }^{13} \mathrm{C}\left\{{ }^{1} \mathrm{H}\right\}$ NMR $\left(100.61 \mathrm{MHz}, \mathrm{CDCl}_{3}\right) \delta: 146.0,136.8,130.8,130.6,127.5,126.0,122.4,121.4$, 120.3, 120.1, 118.4, 111.4, 2.3.

HRMS (EI): calcd. for $\mathrm{C}_{20} \mathrm{H}_{28} \mathrm{~N}_{2} \mathrm{Si}_{2}{ }^{+}: 352.1786\left(\mathrm{~m} / \mathrm{z}[\mathrm{M}]^{+}\right)$, found $352.1790\left(\mathrm{~m} / \mathrm{z}[\mathrm{M}]^{+}\right)$.

3-(naphthalen-1-yl)-1H-indole is obtained as white solid in $89 \%$ isolated yield $(108 \mathrm{mg})$ from 1iodonaphthalene using $0.05 \mathrm{~mol}-\%$ of $\mathrm{Pd}(\mathrm{OAc})_{2}$.

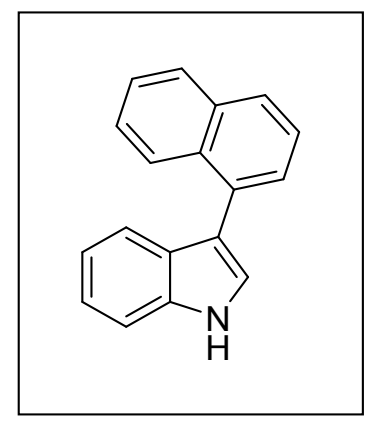

${ }^{1} \mathrm{H}$ NMR $\left(400.13 \mathrm{MHz}, \mathrm{CDCl}_{3}\right) \delta: 8.32(1 \mathrm{H}, \mathrm{br} \mathrm{s}), 8.08(1 \mathrm{H}, \mathrm{d}, J=8.4 \mathrm{~Hz}), 7.93(1 \mathrm{H}, \mathrm{d}, J=8.1 \mathrm{~Hz})$, $7.88(1 \mathrm{H}, \mathrm{d}, J=8.0 \mathrm{~Hz}), 7.61(1 \mathrm{H}, \mathrm{dd}, J=1.4,7.0 \mathrm{~Hz}), 7.58-7.47(4 \mathrm{H}, \mathrm{m}), 7.44-7.37(2 \mathrm{H}, \mathrm{m})$, 7.31 - $7.26(1 \mathrm{H}, \mathrm{m}), 7.17-7.10(1 \mathrm{H}, \mathrm{m})$.

${ }^{13} \mathrm{C}\left\{{ }^{1} \mathrm{H}\right\}$ NMR $\left(100.61 \mathrm{MHz}, \mathrm{CDCl}_{3}\right) \delta: 136.2,134.1,133.1,132.7,128.4,128.0,127.9,127.3$, $126.7,125.8,125.8,125.7,123.6,122.5,120.5,120.2,116.8,111.4$. 
HRMS (ESI): calcd. for $\mathrm{C}_{18} \mathrm{H}_{14} \mathrm{~N}^{+}: 244.1121\left(\mathrm{~m} / \mathrm{z}[\mathrm{M}+\mathrm{H}]^{+}\right)$, found $244.1119\left(\mathrm{~m} / \mathrm{z}[\mathrm{M}+\mathrm{H}]^{+}\right)$.

3-(benzo[b]thiophen-6-yl)-1H-indole is obtained as white solid in $26 \%$ isolated yield (32 mg) from 6-bromobenzo[b]thiophene using $0.05 \mathrm{~mol}-\%$ of $\mathrm{Pd}(\mathrm{OAc})_{2}$. With $0.5 \mathrm{~mol}-\%$ of $\mathrm{Pd}(\mathrm{OAc})_{2}$, the title compound is obtained in $55 \%$ isolated yield $(68 \mathrm{mg})$.

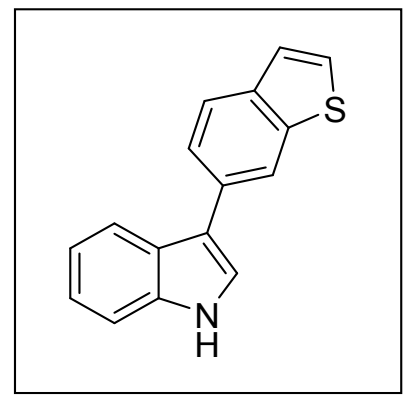

${ }^{1} \mathrm{H}$ NMR $\left(400.13 \mathrm{MHz}, \mathrm{CDCl}_{3}\right)$ ס: $8.26(1 \mathrm{H}, \mathrm{br} \mathrm{s}), 8.20$ - $8.16(1 \mathrm{H}, \mathrm{m}), 8.02-7.98(1 \mathrm{H}, \mathrm{m}), 7.89$ $(1 \mathrm{H}, \mathrm{d}, J=8.2 \mathrm{~Hz}), 7.69(1 \mathrm{H}, \mathrm{dd}, J=1.5,8.2 \mathrm{~Hz}), 7.48-7.40(3 \mathrm{H}, \mathrm{m}), 7.38-7.34(1 \mathrm{H}, \mathrm{m}), 7.31-$ $7.20(2 \mathrm{H}, \mathrm{m})$.

${ }^{13} \mathrm{C}\left\{{ }^{1} \mathrm{H}\right\}$ NMR $\left(100.61 \mathrm{MHz}, \mathrm{CDCl}_{3}\right) \delta: 140.7,138.0,136.8,132.0,126.0,125.8,124.7,123.9$, $123.9,122.7,122.1,120.9,120.6,120.0,118.5,111.6$.

HRMS (ESI): calcd. for $\mathrm{C}_{16} \mathrm{H}_{10} \mathrm{NS}^{-:} 248.0539\left(\mathrm{~m} / \mathrm{z}[\mathrm{M}-\mathrm{H}]^{+}\right)$, found $248.0548\left(\mathrm{~m} / \mathrm{z}[\mathrm{M}-\mathrm{H}]^{+}\right)$.

3-(ferrocenyl)-1H-indole is obtained as orange solid in $8 \% \mathrm{GC}$ yield from bromoferrocene using $0.05 \mathrm{~mol}-\%$ of $\mathrm{Pd}(\mathrm{OAc})_{2}$. With $0.5 \mathrm{~mol}-\%$ of $\mathrm{Pd}(\mathrm{OAc})_{2}$, the title compound is obtained in $26 \%$ isolated yield $(39 \mathrm{mg})$.

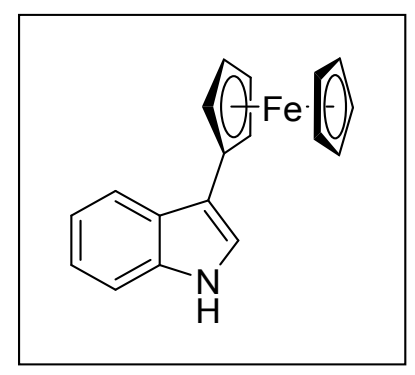

${ }^{1} \mathrm{H}$ NMR $\left(400.13 \mathrm{MHz}, \mathrm{CDCl}_{3}\right)$ ס: $8.00-7.93(1 \mathrm{H}, \mathrm{m}), 7.87(1 \mathrm{H}, \mathrm{br} \mathrm{s}), 7.42-7.33(1 \mathrm{H}, \mathrm{m}), 7.25-$ $7.15(2 \mathrm{H}, \mathrm{m}), 7.03(1 \mathrm{H}, \mathrm{s}), 4.78(2 \mathrm{H}, \mathrm{s}), 4.42(2 \mathrm{H}, \mathrm{s}), 4.16(5 \mathrm{H}, \mathrm{s})$.

${ }^{13} \mathrm{C}\left\{{ }^{1} \mathrm{H}\right\}$ NMR $\left(100.61 \mathrm{MHz}, \mathrm{CDCl}_{3}\right) \delta: 136.8,126.3,122.4,121.4,120.7,119.8,114.8,111.4,70.7$, $69.6,68.1,66.4$. 
HRMS (ESI): calcd. for $\mathrm{C}_{18} \mathrm{H}_{15} \mathrm{FeN}^{+:} 301.0548\left(\mathrm{~m} / \mathrm{z}[\mathrm{M}]^{+}\right)$, found $301.0546\left(\mathrm{~m} / \mathrm{z}[\mathrm{M}]^{+}\right)$.

2-methyl-3-phenyl-1H-indole: is obtained in 4\% GC yield from iodobenzene and 2\% GC yield from bromobenzene using $0.05 \mathrm{~mol}-\%$ of $\mathrm{Pd}(\mathrm{OAc})_{2}$. With $0.5 \mathrm{~mol}-\%$ of $\mathrm{Pd}(\mathrm{OAc})_{2}$, the title compound is obtained in $19 \%$ and $9 \%$ GC yield, respectively. When the reaction is conducted using 2 mol- $\%$ of Pd-catalyst, 2-methyl-3-phenyl-1H-indole can be obtained as a white powder in $80 \%$ isolated yield (83 $\mathrm{mg}$ ) from iodobenzene.

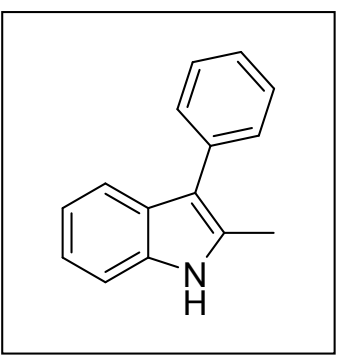

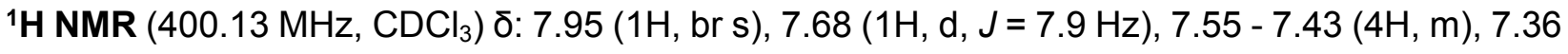
- $7.28(2 \mathrm{H}, \mathrm{m}), 7.21-7.09(2 \mathrm{H}, \mathrm{m}), 2.52(3 \mathrm{H}, \mathrm{s})$.

${ }^{13} \mathrm{C}\left\{{ }^{1} \mathrm{H}\right\}$ NMR $\left(100.61 \mathrm{MHz}, \mathrm{CDCl}_{3}\right) \delta: 135.6,135.4,131.5,129.6,128.6,128.0,125.9,121.7$, 120.1, 118.9, 114.7, 110.4, 12.7.

HRMS (ESI): calcd. for $\mathrm{C}_{15} \mathrm{H}_{13} \mathrm{~N}: 206.0970\left(\mathrm{~m} / \mathrm{z}[\mathrm{M}+\mathrm{H}]^{+}\right)$, found $206.0977\left(\mathrm{~m} / \mathrm{z}[\mathrm{M}+\mathrm{H}]^{+}\right)$.

5-methyl-3-phenyl-1H-indole: the GC-MS results show a second but minor peak corresponding to the same $\mathrm{m} / \mathrm{z}$ value as the product peak (see Figure S3). As this by-product could not be isolated the selectivity towards the C-3 product was determined by GC-FID analysis. 5-methyl-3-phenyl$1 \mathrm{H}$-indole is obtained as an orange solid in $90 \%$ isolated yield $(93 \mathrm{mg})$ from iodobenzene and $88 \%$ isolated yield $(91 \mathrm{mg})$ from bromobenzene with a selectivity of $99 \%$ in both cases using $0.05 \mathrm{~mol}-\%$ of $\mathrm{Pd}(\mathrm{OAc})_{2}$..

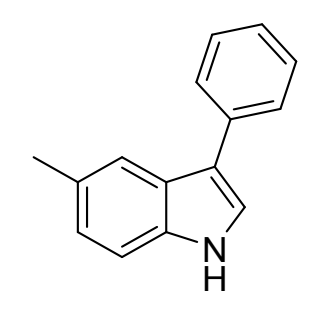




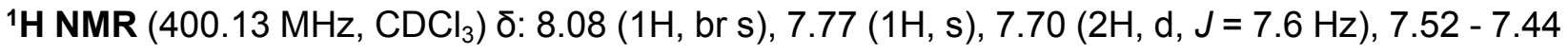
$(2 \mathrm{H}, \mathrm{m}), 7.36-7.28(3 \mathrm{H}, \mathrm{m}), 7.11(1 \mathrm{H}, \mathrm{d}, J=8.2 \mathrm{~Hz}), 2.52(3 \mathrm{H}, \mathrm{s})$.

${ }^{13} \mathrm{C}\left\{{ }^{1} \mathrm{H}\right\}$ NMR $\left(100.61 \mathrm{MHz}, \mathrm{CDCl}_{3}\right) \delta: 135.9,135.1,129.7,128.9,127.6,126.1,126.01,124.1$, 122.1, 119.5, 118.0, 111.2, 21.8.

HRMS (ESI): calcd. for $\mathrm{C}_{15} \mathrm{H}_{13} \mathrm{~N}: 208.1126\left(\mathrm{~m} / \mathrm{z}[\mathrm{M}+\mathrm{H}]^{+}\right)$, found $208.1111\left(\mathrm{~m} / \mathrm{z}[\mathrm{M}+\mathrm{H}]^{+}\right)$.

5-fluoro-3-phenyl-1H-indole is obtained as an orange oil in $56 \%$ isolated yield $(59 \mathrm{mg})$ from iodobenzene and $25 \%$ isolated yield $(26 \mathrm{mg})$ from bromobenzene using $0.05 \mathrm{~mol}-\%$ of $\mathrm{Pd}(\mathrm{OAc})_{2}$. With $0.5 \mathrm{~mol}-\%$ of $\mathrm{Pd}(\mathrm{OAc})_{2}$, the title compound is obtained in $50 \%$ isolated yield $(53 \mathrm{mg})$ from bromobenzene.

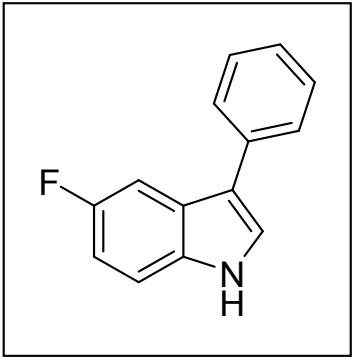

${ }^{1} \mathrm{H}$ NMR (400.13 MHz, $\left.\mathrm{CDCl}_{3}\right)$ ס: $8.31(1 \mathrm{H}, \mathrm{br} \mathrm{s}), 7.67$ - $7.57(3 \mathrm{H}, \mathrm{m}), 7.50-7.43(2 \mathrm{H}, \mathrm{m}), 7.40$ $(1 \mathrm{H}, \mathrm{d}, J=2.5 \mathrm{~Hz}), 7.37-7.28(2 \mathrm{H}, \mathrm{m}), 7.01(1 \mathrm{H}, \mathrm{dt}, J=2.5,8.9 \mathrm{~Hz})$.

${ }^{13} \mathrm{C}\left\{{ }^{1} \mathrm{H}\right\}$ NMR $\left(100.61 \mathrm{MHz}, \mathrm{CDCl}_{3}\right) \delta: 158.5(\mathrm{~d}, J=235 \mathrm{~Hz}), 135.2,133.3,129.0,127.4,126.3$, 126.2, 123.6, 118.6 (d, $J=4.7 \mathrm{~Hz}), 112.2$ (d, $J=9.8 \mathrm{~Hz}), 111.0(\mathrm{~d}, J=26.4 \mathrm{~Hz}), 104.9(\mathrm{~d}, J=$ $24.2 \mathrm{~Hz})$.

HRMS (ESI): calcd. for $\mathrm{C}_{14} \mathrm{H}_{10} \mathrm{FN}: 210.0719\left(\mathrm{~m} / \mathrm{z}[\mathrm{M}-\mathrm{H}]^{-}\right)$, found $210.0732\left(\mathrm{~m} / \mathrm{z}[\mathrm{M}-\mathrm{H}]^{-}\right)$.

5-chloro-3-phenyl-1H-indole is obtained as an orange powder in $78 \%$ isolated yield $(89 \mathrm{mg})$ from iodobenzene and $43 \%$ isolated yield $\left(49 \mathrm{mg}\right.$ ) from bromobenzene using $0.05 \mathrm{~mol}-\%$ of $\mathrm{Pd}(\mathrm{OAc})_{2}$. With $0.5 \mathrm{~mol}-\%$ of $\mathrm{Pd}(\mathrm{OAc})_{2}$, the title compound is obtained in $62 \%$ isolated yield $(70 \mathrm{mg})$ from bromobenzene.

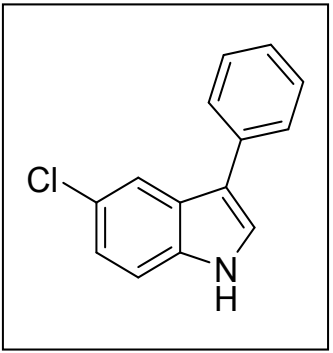




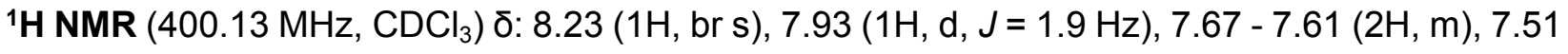
- $7.45(2 \mathrm{H}, \mathrm{m}), 7.38$ - $7.30(3 \mathrm{H}, \mathrm{m}), 7.22(1 \mathrm{H}, \mathrm{dd}, J=1.9,8.6 \mathrm{~Hz})$.

${ }^{13} \mathrm{C}\left\{{ }^{1} \mathrm{H}\right\}$ NMR $\left(100.61 \mathrm{MHz}, \mathrm{CDCl}_{3}\right) \delta: 135.1,134.9,129.0,127.6,127.0,126.4,126.2,123.1$, $122.9,119.4,118.3,112.5$.

HRMS (ESI): calcd. for $\mathrm{C}_{14} \mathrm{H}_{10} \mathrm{CIN}: 226.0424\left(\mathrm{~m} / \mathrm{z}[\mathrm{M}-\mathrm{H}]^{-}\right)$, found $226.0425\left(\mathrm{~m} / \mathrm{z}[\mathrm{M}-\mathrm{H}]^{-}\right)$.

5-bromo-3-phenyl-1H-indole is obtained as a colorless oil in $44 \%$ isolated yield $(60 \mathrm{mg})$ from iodobenzene using 0.05 mol- $\%$ of $\mathrm{Pd}(\mathrm{OAc})_{2}$. With $0.5 \mathrm{~mol}-\%$ of $\mathrm{Pd}(\mathrm{OAc})_{2}$, the title compound is obtained in $55 \%$ isolated yield $(75 \mathrm{mg})$ from iodobenzene.

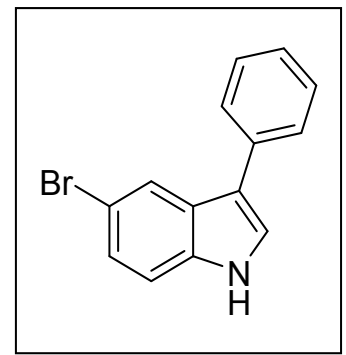

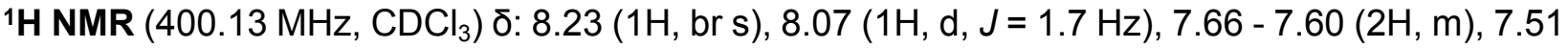
- $7.44(2 \mathrm{H}, \mathrm{m}), 7.37$ - $7.31(3 \mathrm{H}, \mathrm{m}), 7.29(1 \mathrm{H}, \mathrm{d}, J=8.6 \mathrm{~Hz})$.

${ }^{13} \mathrm{C}\left\{{ }^{1} \mathrm{H}\right\}$ NMR $\left(100.61 \mathrm{MHz}, \mathrm{CDCl}_{3}\right) \delta: 135.3,134.9,129.0,127.6,127.6,126.5,125.4,123.0$, $122.5,118.2,113.8,112.9$.

HRMS (ESI): calcd. for $\mathrm{C}_{14} \mathrm{H}_{10} \mathrm{BrN}: 269.9918$ (m/z [M-H]-), found $269.9920\left(\mathrm{~m} / \mathrm{z}[\mathrm{M}-\mathrm{H}]^{-}\right)$.

5-methoxy-3-phenyl-1H-indole is obtained as a red oil in $38 \%$ isolated yield $(42 \mathrm{mg})$ from iodobenzene and $14 \%$ GC yield from bromobenzene using 0.05 mol- $\%$ of $\mathrm{Pd}(\mathrm{OAc})_{2}$. With $0.5 \mathrm{~mol}-$ $\%$ of $\mathrm{Pd}(\mathrm{OAc})_{2}$, the title compound is obtained in $51 \%(57 \mathrm{mg})$ and $46 \%(51 \mathrm{mg})$ isolated yield from iodobenzene or bromobenzene, respectively.

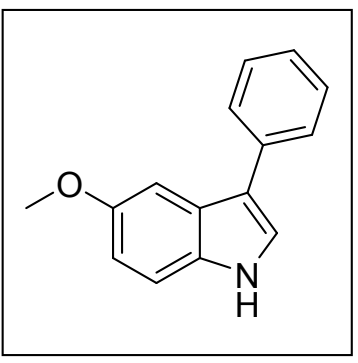


${ }^{1} \mathrm{H}$ NMR (400.13 MHz, $\left.\mathrm{CDCl}_{3}\right)$ ס: $8.13(1 \mathrm{H}, \mathrm{br} \mathrm{s}), 7.71-7.65(2 \mathrm{H}, \mathrm{m}), 7.52-7.45(2 \mathrm{H}, \mathrm{m}), 7.43$ $(1 \mathrm{H}, \mathrm{d}, J=2.3 \mathrm{~Hz}), 7.36-7.28(3 \mathrm{H}, \mathrm{m}), 6.95(1 \mathrm{H}, \mathrm{dd}, J=2.4,8.8 \mathrm{~Hz}), 3.9(3 \mathrm{H}, \mathrm{s})$.

${ }^{13} \mathrm{C}\left\{{ }^{1} \mathrm{H}\right\}$ NMR $\left(100.61 \mathrm{MHz}, \mathrm{CDCl}_{3}\right) \delta: 154.8,135.8,131.9,128.9,127.5,126.3,126.0,122.8$, 118.2, 112.8, 112.2, 101.7, 56.1.

HRMS (ESI): calcd. for $\mathrm{C}_{15} \mathrm{H}_{13} \mathrm{NO}: 224.1075\left(\mathrm{~m} / \mathrm{z}[\mathrm{M}+\mathrm{H}]^{+}\right)$, found $224.01069\left(\mathrm{~m} / \mathrm{z}[\mathrm{M}+\mathrm{H}]^{+}\right)$.

3-phenyl-5-(4,4,5,5-tetramethyl-1,3,2-dioxaborolan-2-yl)-1H-indole is obtained as a white solid in $31 \%$ isolated yield $(49 \mathrm{mg}$ ) from iodobenzene and 6\% GC yield from bromobenzene using 0.05 mol- $\%$ of $\mathrm{Pd}(\mathrm{OAc})_{2}$. With $0.5 \mathrm{~mol}-\%$ of $\mathrm{Pd}(\mathrm{OAc})_{2}$, the title compound is obtained in $47 \%$ isolated yield from iodobenzene ( $75 \mathrm{mg}$ ) and $13 \% \mathrm{GC}$ yield from bromobenzene.

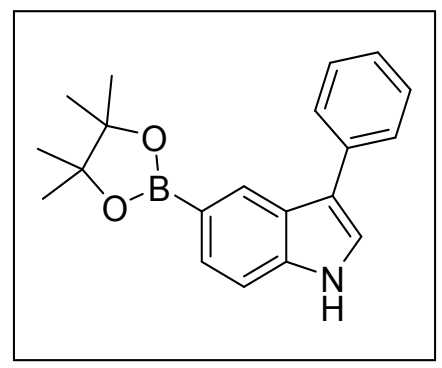

${ }^{1} \mathrm{H}$ NMR $\left(400.13 \mathrm{MHz}, \mathrm{CDCl}_{3}\right)$ ס: $8.47(1 \mathrm{H}, \mathrm{s}), 8.35(1 \mathrm{H}, \mathrm{br} \mathrm{s}), 7.76$ - $7.66(3 \mathrm{H}, \mathrm{m}), 7.48$ - $7.39(3 \mathrm{H}$, m), 7.34 - $7.27(2 \mathrm{H}, \mathrm{m}), 1.39(12 \mathrm{H}, \mathrm{s})$.

${ }^{13} \mathrm{C}\left\{{ }^{1} \mathrm{H}\right\}$ NMR $\left(100.61 \mathrm{MHz}, \mathrm{CDCl}_{3}\right) \delta: 138.8,135.5,128.9,128.7,127.9,127.7,126.2,125.7$, $121.9,119.1,111.0,83.7,25.0$ (one signal missing, but in coherence with literature ${ }^{7}$ ).

HRMS (ESI): calcd. for $\mathrm{C}_{20} \mathrm{H}_{22} \mathrm{BNO}_{2}: 318.1665$ (m/z [M-H]-), found $318.1672\left(\mathrm{~m} / \mathrm{z}[\mathrm{M}-\mathrm{H}]^{-}\right)$.

7-methyl-3-phenyl-1H-indole is obtained as a red oil in $60 \%$ isolated yield $(62 \mathrm{mg})$ from iodobenzene and $23 \%$ isolated yield $\left(24 \mathrm{mg}\right.$ ) from bromobenzene using $0.05 \mathrm{~mol}-\%$ of $\mathrm{Pd}(\mathrm{OAc})_{2}$. With $0.5 \mathrm{~mol}-\%$ of $\mathrm{Pd}(\mathrm{OAc})_{2}$, the title compound is obtained in $71 \%$ isolated yield $(73 \mathrm{mg})$ from bromobenzene.

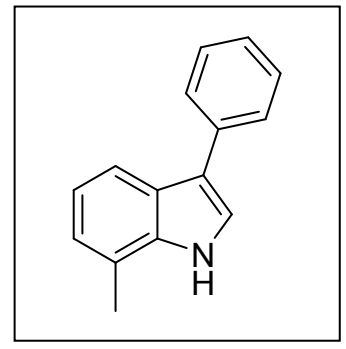


${ }^{1} \mathrm{H}$ NMR $\left(400.13 \mathrm{MHz}, \mathrm{CDCl}_{3}\right) \delta: 8.17(1 \mathrm{H}, \mathrm{br} \mathrm{s}), 7.80(1 \mathrm{H}, \mathrm{d}, J=7.9 \mathrm{~Hz}), 7.70-7.66(2 \mathrm{H}, \mathrm{m}), 7.48$ - $7.42(2 \mathrm{H}, \mathrm{m}), 7.39(1 \mathrm{H}, \mathrm{d}, \mathrm{J}=2.5 \mathrm{~Hz}), 7.32$ - $7.27(1 \mathrm{H}, \mathrm{m}), 7.16-7.04(2 \mathrm{H}, \mathrm{m}), 2.54(3 \mathrm{H}, \mathrm{s})$.

${ }^{13} \mathrm{C}\left\{{ }^{1} \mathrm{H}\right\}$ NMR $\left(100.61 \mathrm{MHz}, \mathrm{CDCl}_{3}\right) \delta: 136.4,135.9,128.9,127.7,126.1,125.5,123.1,121.6$, 120.7, 120.6, 119.1, 117.7, 16.8 .

HRMS (ESI): calcd. for $\mathrm{C}_{15} \mathrm{H}_{13} \mathrm{~N}: 208.1126\left(\mathrm{~m} / \mathrm{z}[\mathrm{M}+\mathrm{H}]^{+}\right)$, found $208.1123\left(\mathrm{~m} / \mathrm{z}[\mathrm{M}+\mathrm{H}]^{+}\right)$.

3,7-diphenyl-1H-indole is obtained as red oil in $72 \%$ isolated yield $(97 \mathrm{mg})$ from iodobenzene and $66 \%$ isolated yield $\left(89 \mathrm{mg}\right.$ ) from bromobenzene using $0.05 \mathrm{~mol}-\%$ of $\mathrm{Pd}(\mathrm{OAc})_{2}$.

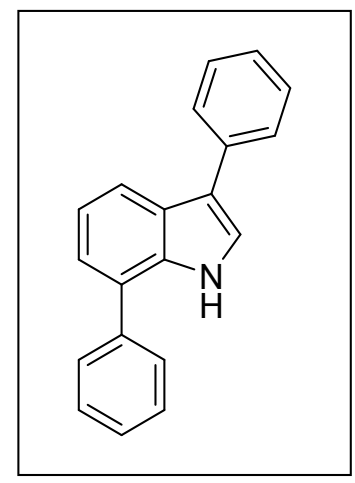

${ }^{1} \mathrm{H}$ NMR $\left(400.13 \mathrm{MHz}, \mathrm{CDCl}_{3}\right)$ ठ: $8.47(1 \mathrm{H}, \mathrm{br} \mathrm{s}), 8.02$ - $7.94(1 \mathrm{H}, \mathrm{m}), 7.76-7.64(4 \mathrm{H}, \mathrm{m}), 7.60$ $7.42(5 \mathrm{H}, \mathrm{m}), 7.40-7.28(4 \mathrm{H}, \mathrm{m})$.

${ }^{13} \mathrm{C}\left\{{ }^{1} \mathrm{H}\right\}$ NMR $\left(100.61 \mathrm{MHz}, \mathrm{CDCl}_{3}\right) \delta: 139.1,135.7,134.7,129.3,128.9,128.4,127.7,127.7$, $126.3,126.2,126.1,122.5,122.1,121.0,119.2,119.0$.

HRMS (ESI): calcd. for $\mathrm{C}_{20} \mathrm{H}_{14} \mathrm{~N}: 268.1126\left(\mathrm{~m} / \mathrm{z}[\mathrm{M}-\mathrm{H}]^{-}\right)$, found $268.1132\left(\mathrm{~m} / \mathrm{z}[\mathrm{M}-\mathrm{H}]^{-}\right)$. 


\section{NMR Spectra of C-3 arylated indoles}
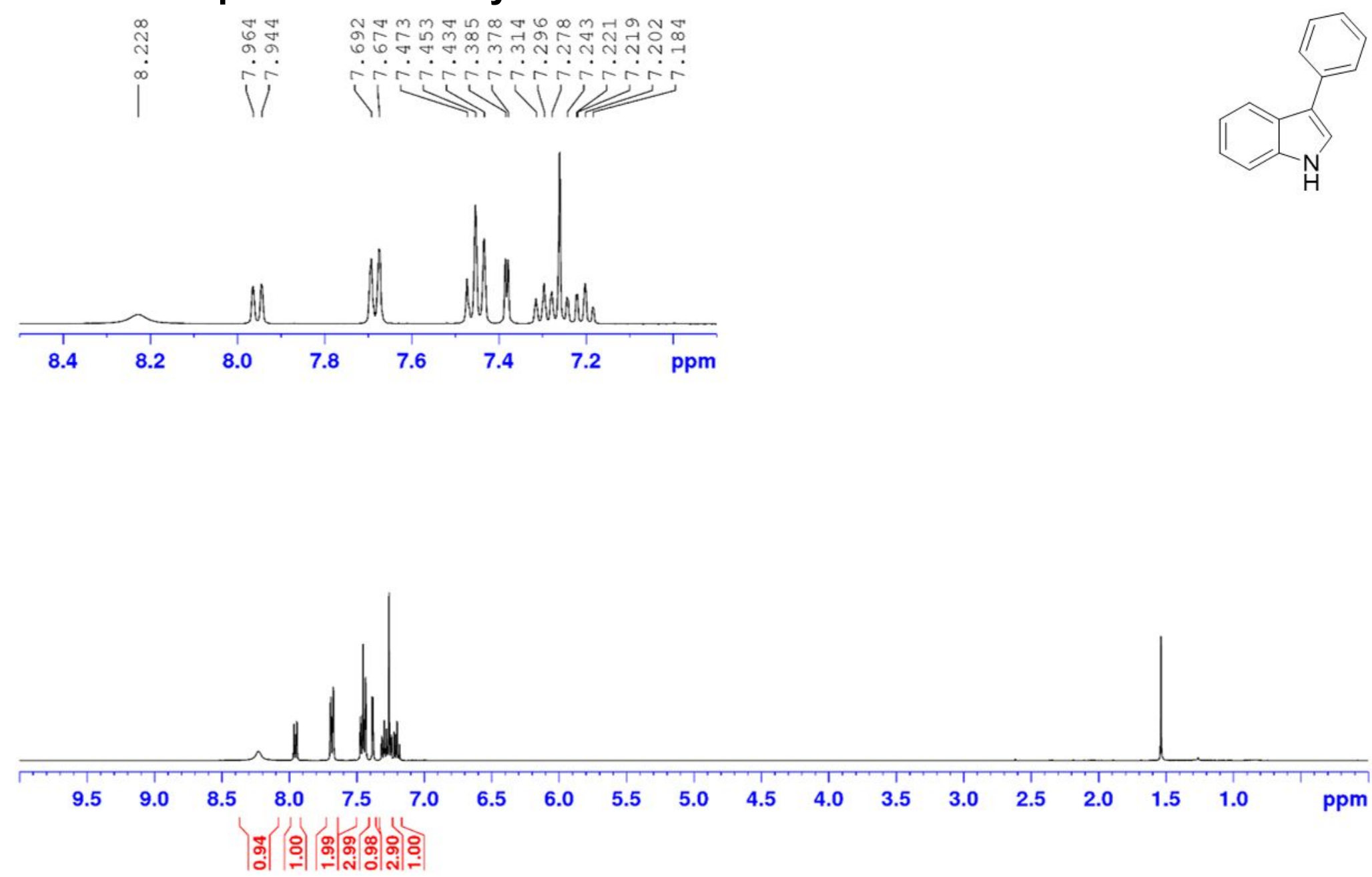

Figure S4: ${ }^{1} \mathrm{H}$ NMR spectrum of 3-phenyl-1H-indole $\left(400.13 \mathrm{MHz}, \mathrm{CDCl}_{3}\right)$. 


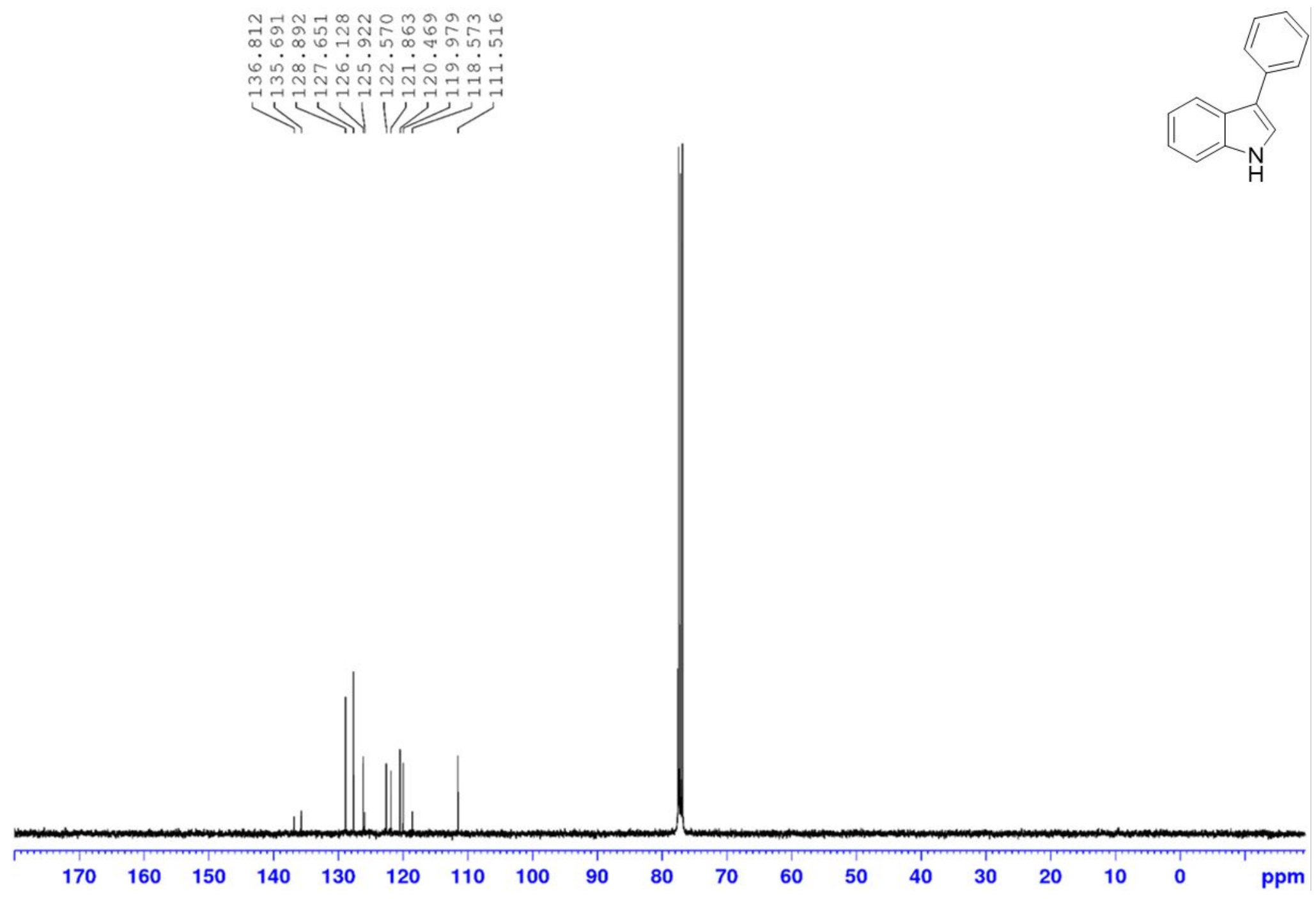

Figure S5: ${ }^{13} \mathrm{C}$ NMR spectrum of 3-phenyl-1 $H$-indole $\left(100.61 \mathrm{MHz}, \mathrm{CDCl}_{3}\right)$. 


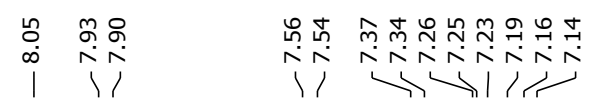

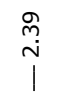
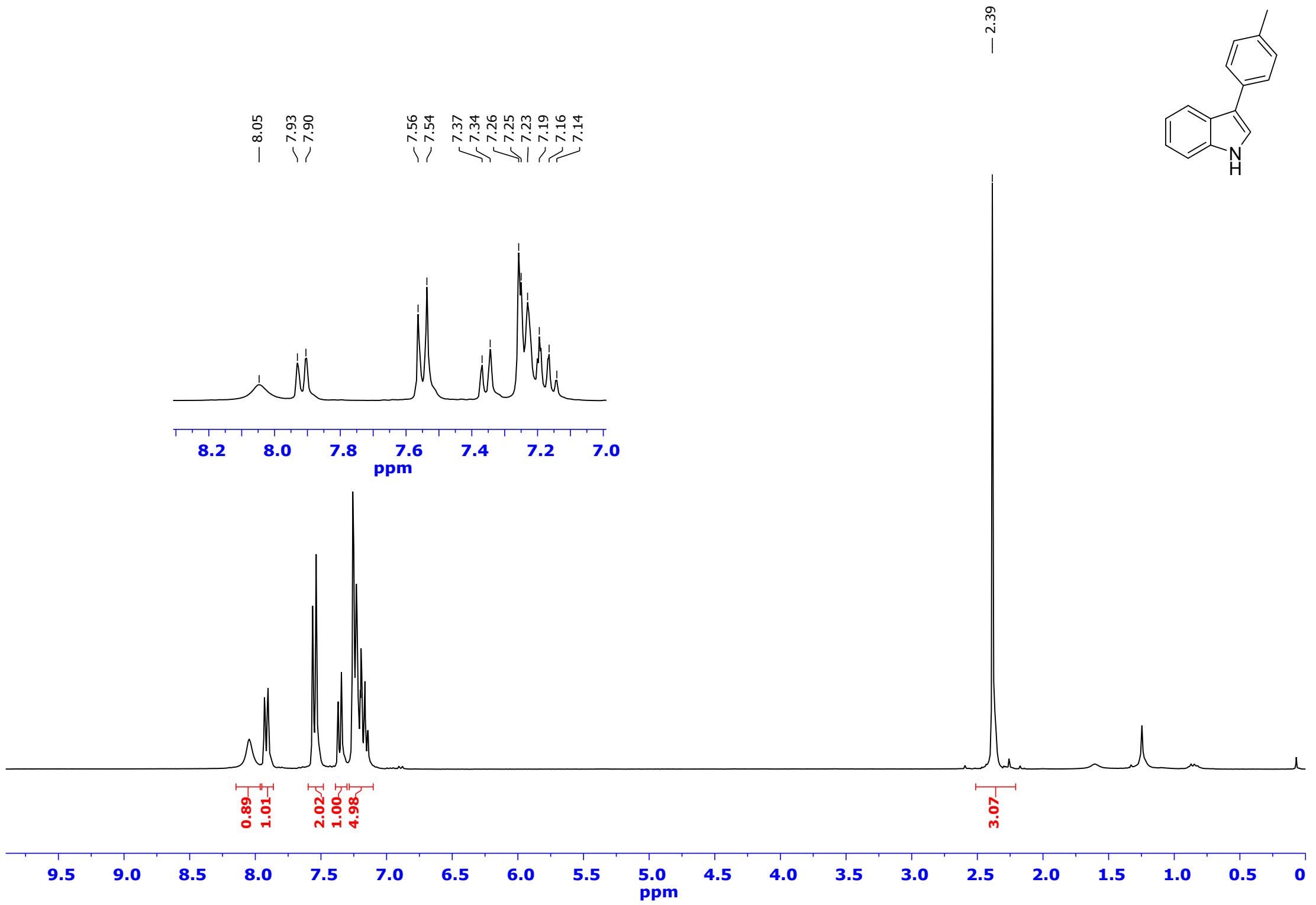

Figure S6: ${ }^{1} \mathrm{H}$ NMR spectrum of 3-(p-tolyl)-1 $\mathrm{H}$-indole $\left(300.13 \mathrm{MHz}, \mathrm{CDCl}_{3}\right)$.

S33 


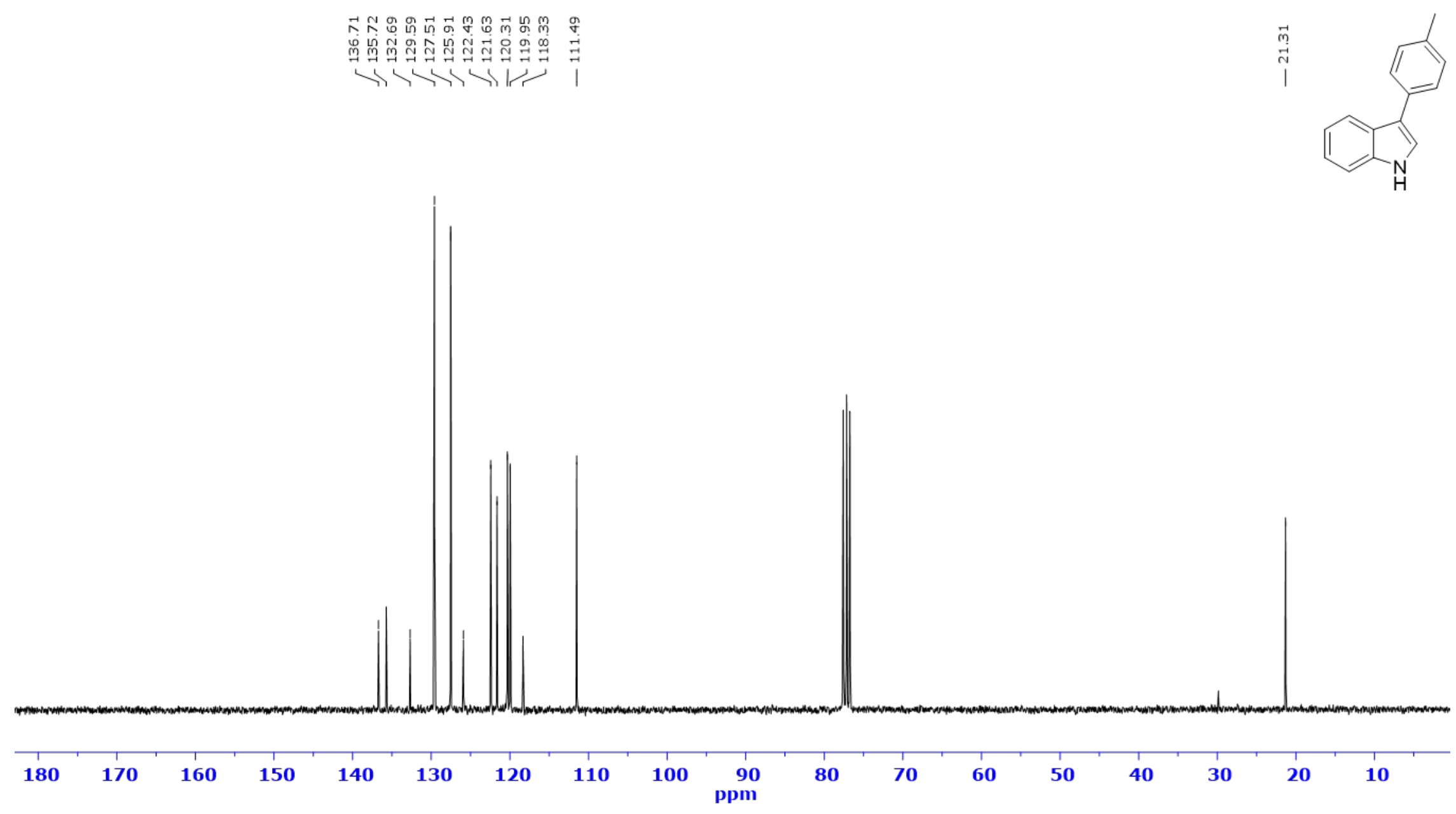

Figure S7: ${ }^{13} \mathrm{C}$ NMR spectrum of $3-\left(p\right.$-tolyl)-1H-indole $\left(75.47 \mathrm{MHz}, \mathrm{CDCl}_{3}\right)$. 

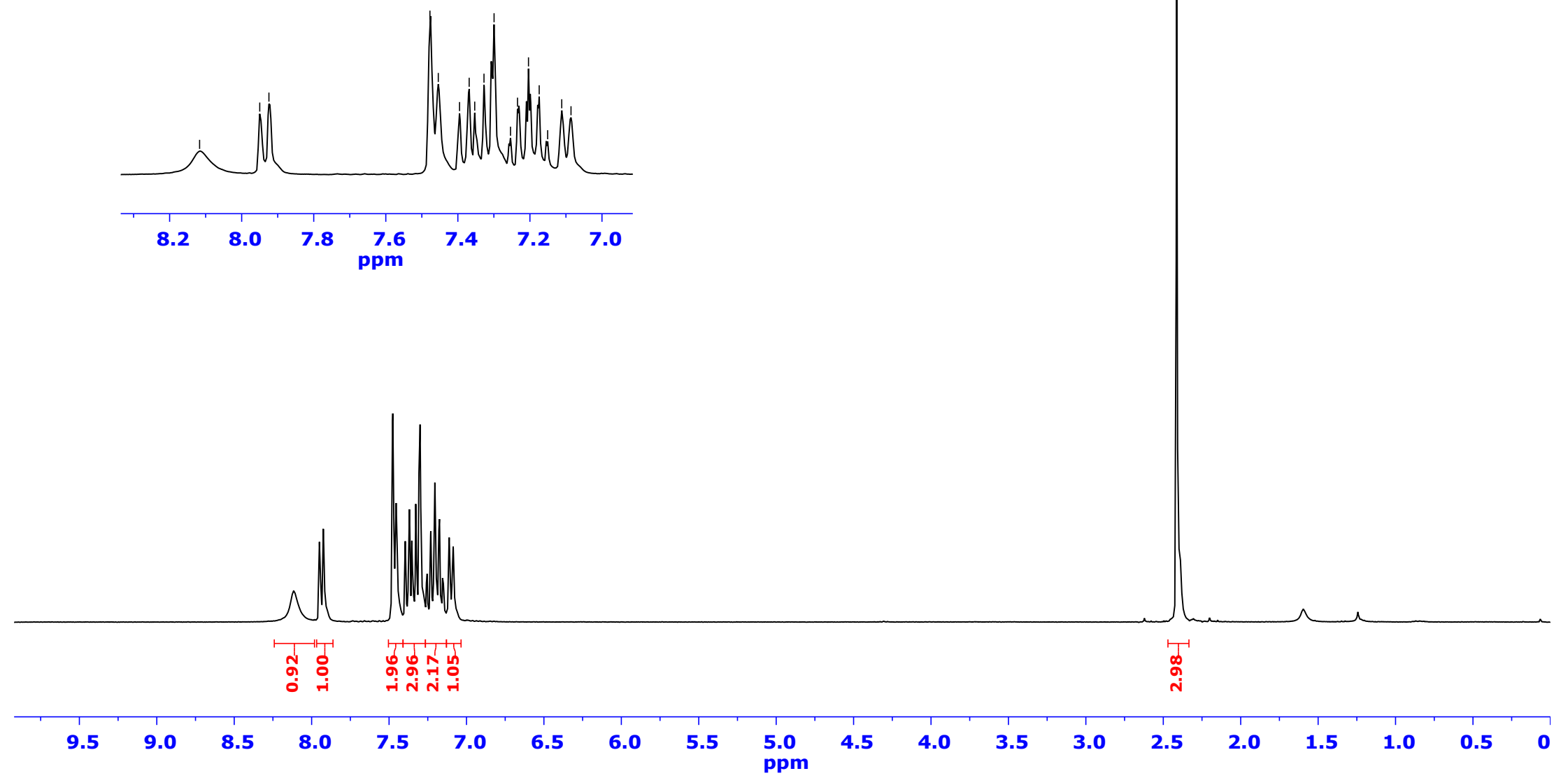

Figure S8: ${ }^{1} \mathrm{H}$ NMR spectrum of 3-( $m$-tolyl)-1 $1 \mathrm{H}$-indole $\left(300.13 \mathrm{MHz}, \mathrm{CDCl}_{3}\right)$. 

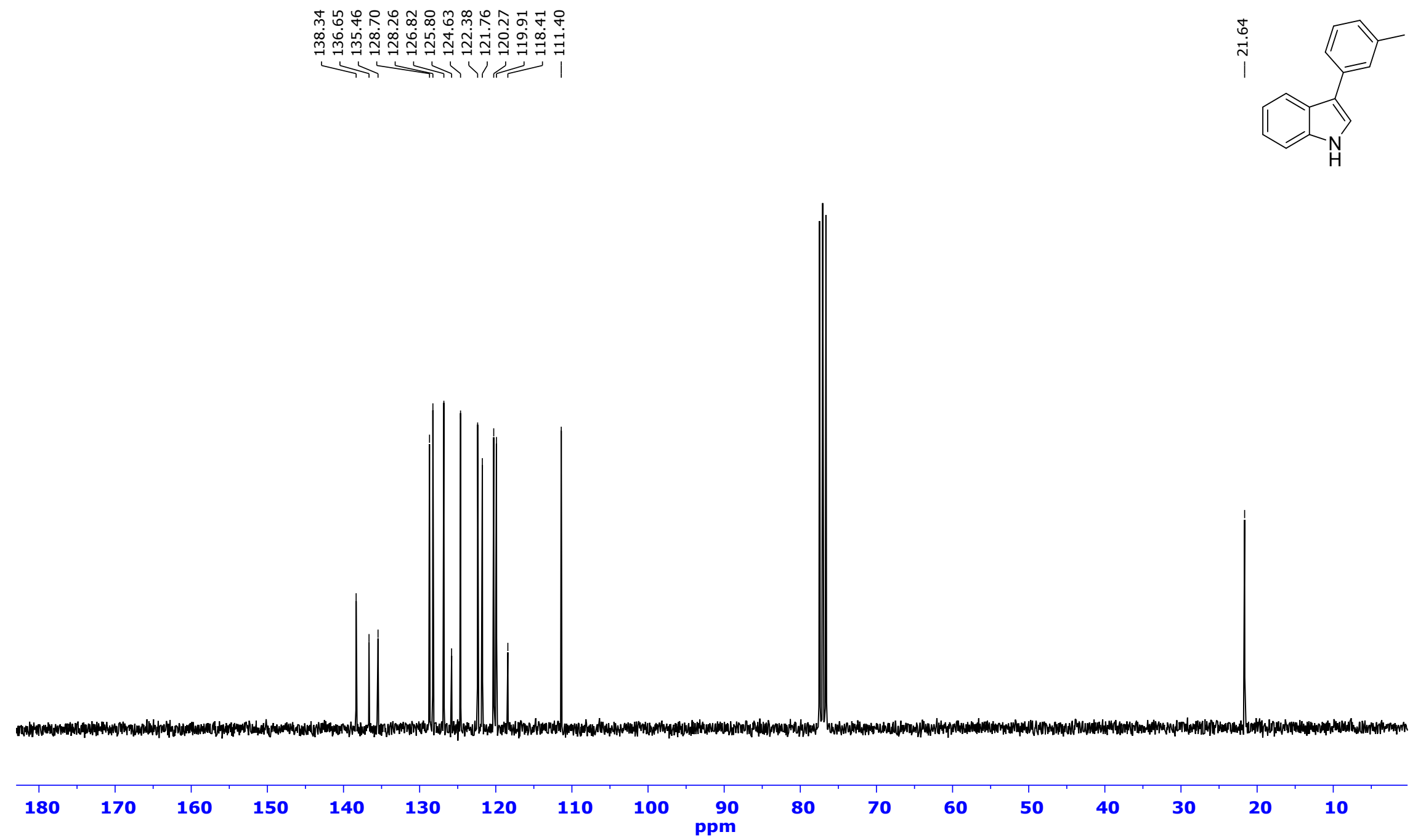

Figure S9: ${ }^{13} \mathrm{C}$ NMR spectrum of 3-(m-tolyl)-1H-indole $\left(75.47 \mathrm{MHz}, \mathrm{CDCl}_{3}\right)$. 


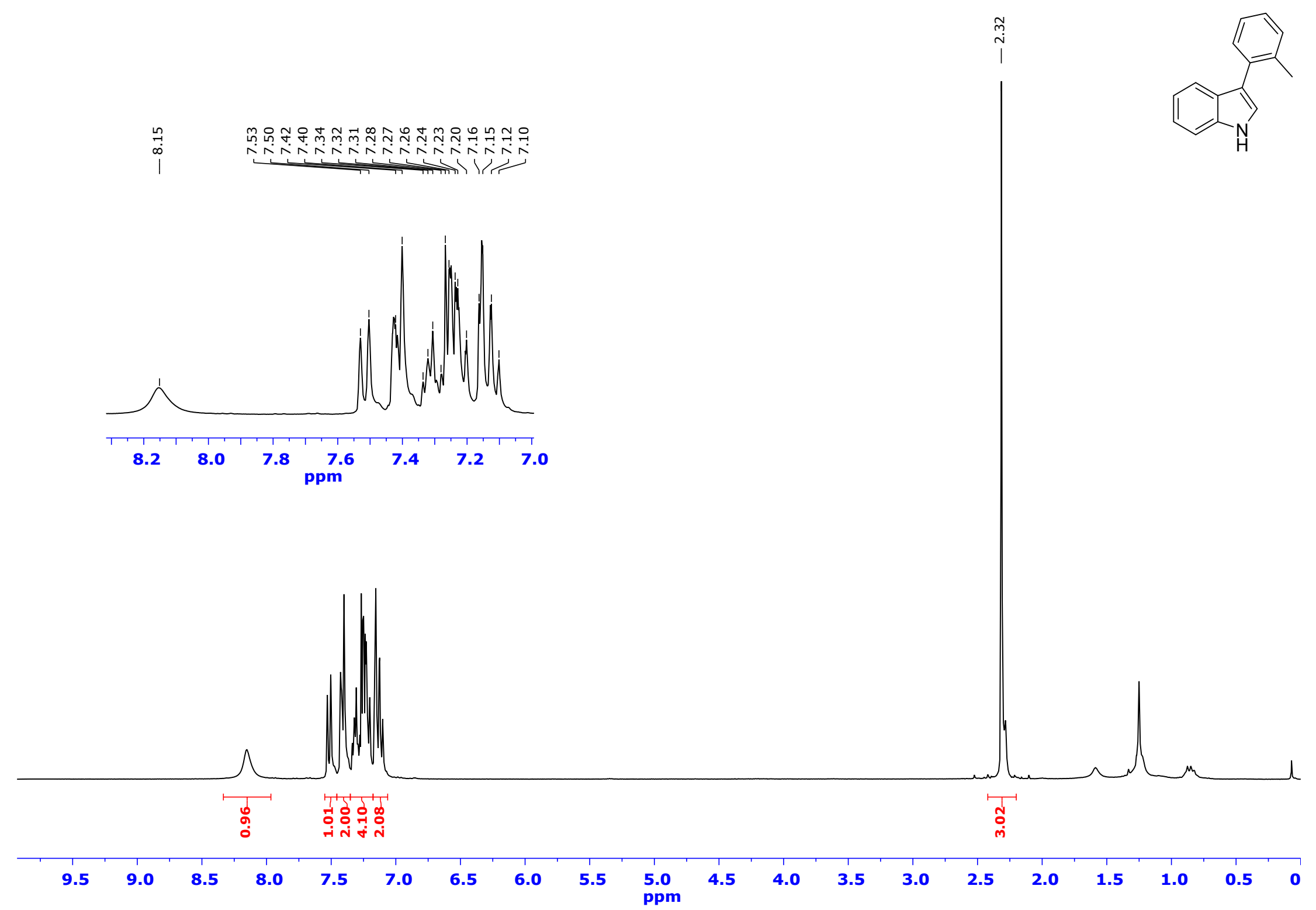

Figure S10: ${ }^{1} \mathrm{H}$ NMR spectrum of 3-(o-tolyl)-1H-indole $\left(300.13 \mathrm{MHz}, \mathrm{CDCl}_{3}\right)$. 


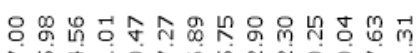

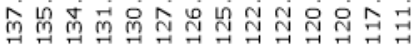

को
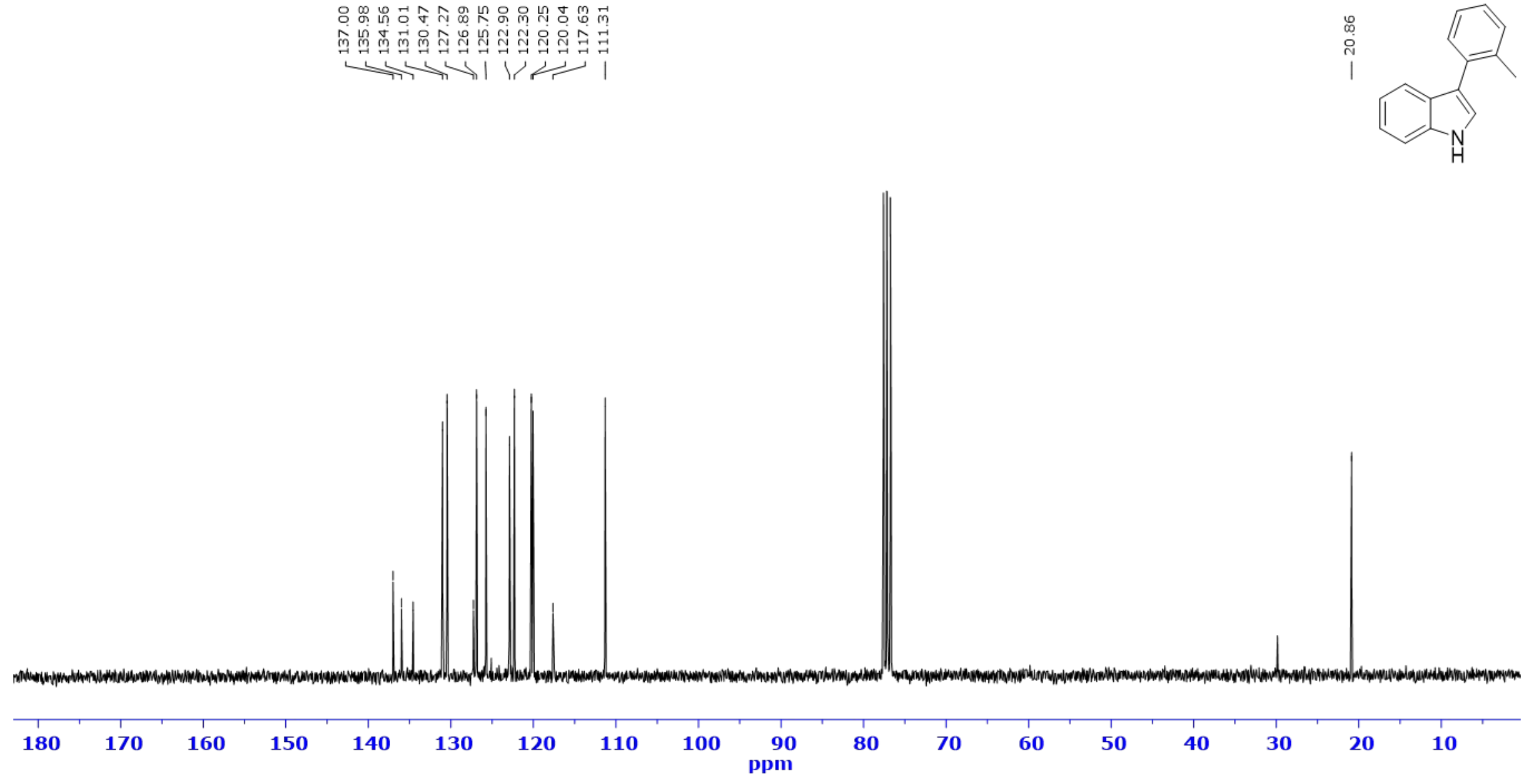

Figure S11: ${ }^{13} \mathrm{C}$ NMR spectrum of 3-(o-tolyl)-1H-indole $\left(75.47 \mathrm{MHz}, \mathrm{CDCl}_{3}\right.$ ). 


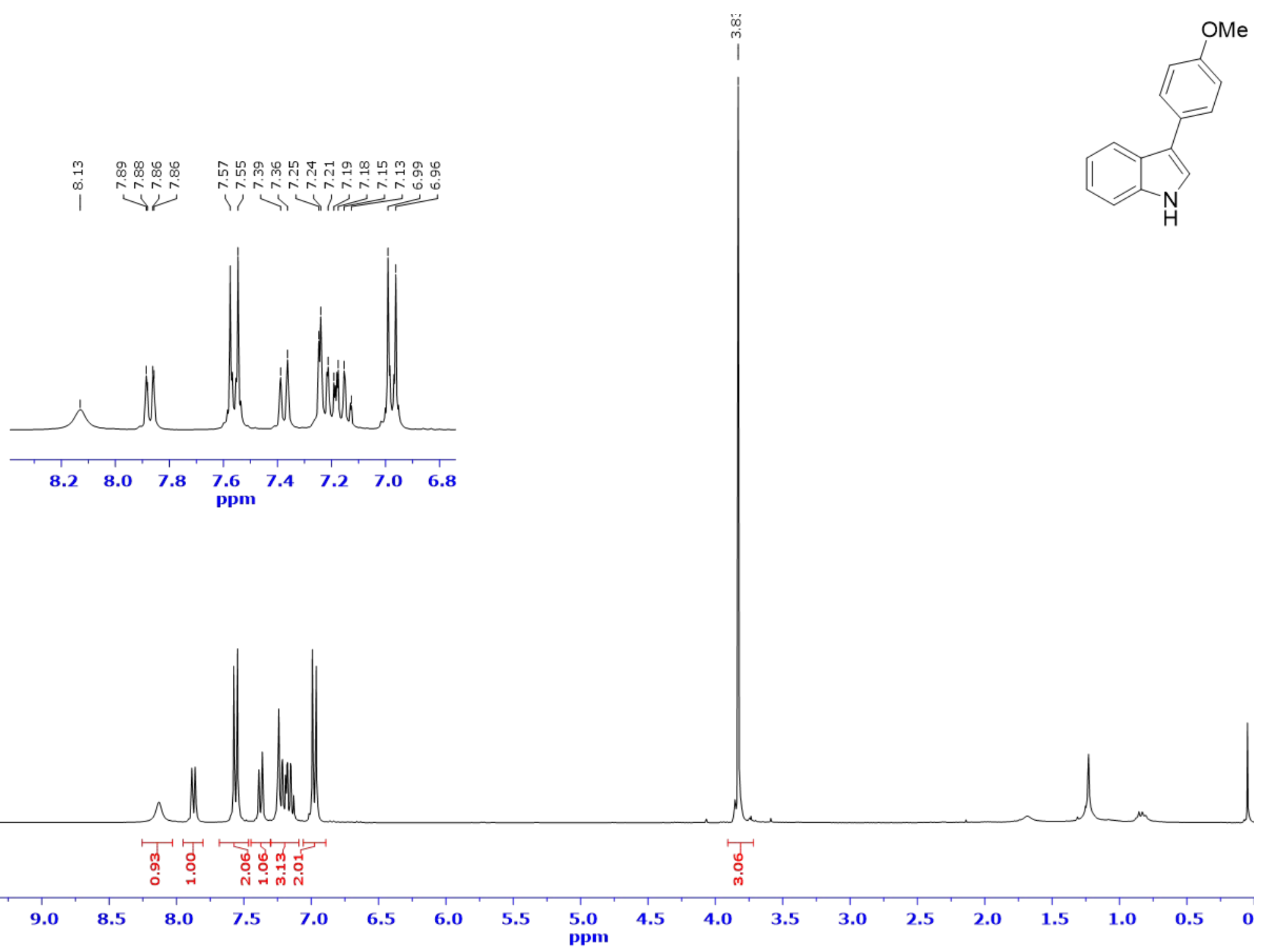

Figure S12: ${ }^{1} \mathrm{H}$ NMR spectrum of 3-(4-methoxyphenyl)-1 $\mathrm{H}$-indole $\left(300.13 \mathrm{MHz}, \mathrm{CDCl}_{3}\right)$. 


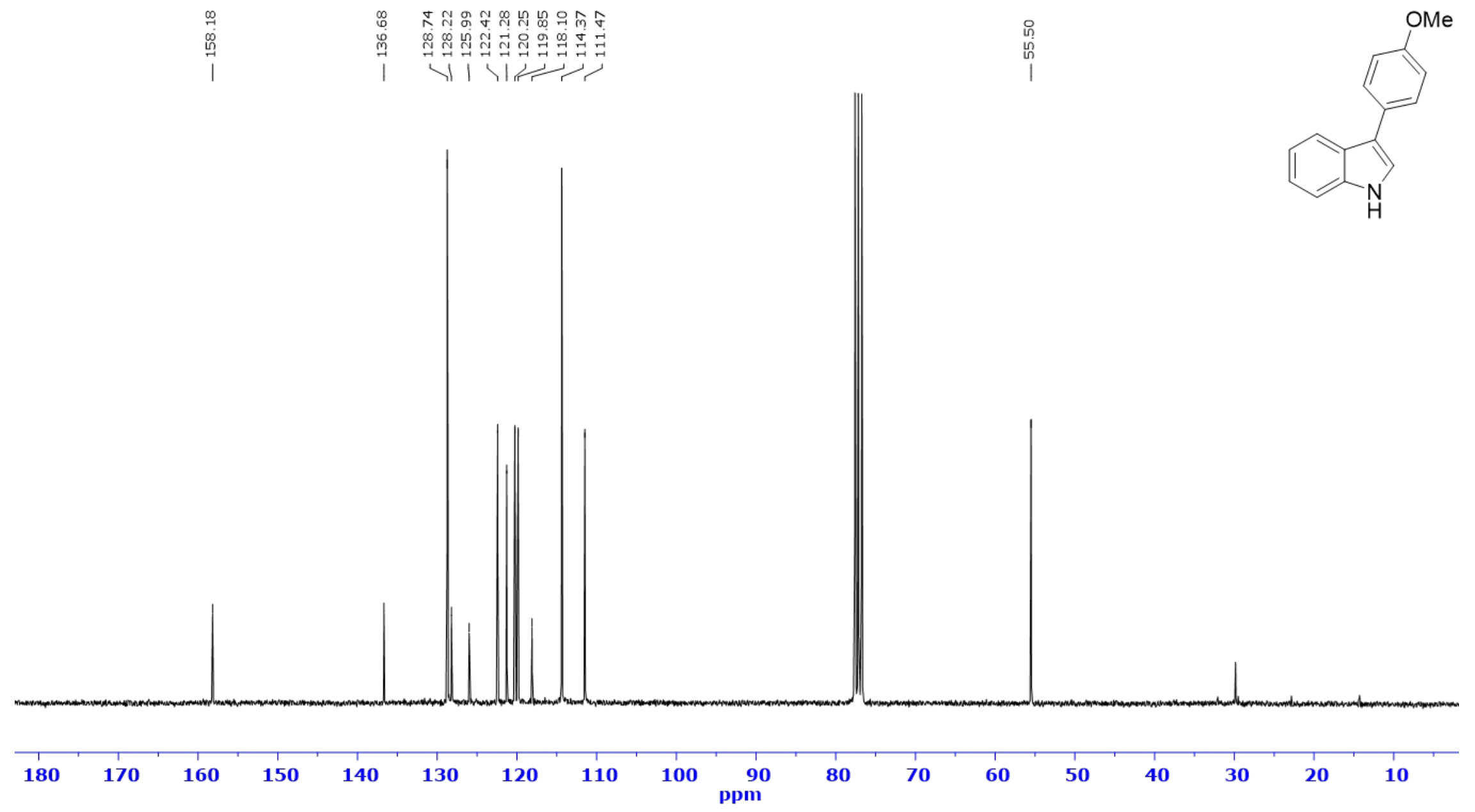

Figure S13: ${ }^{13} \mathrm{C}$ NMR spectrum of 3-(4-methoxyphenyl)-1H-indole $\left(75.47 \mathrm{MHz}, \mathrm{CDCl}_{3}\right)$. 


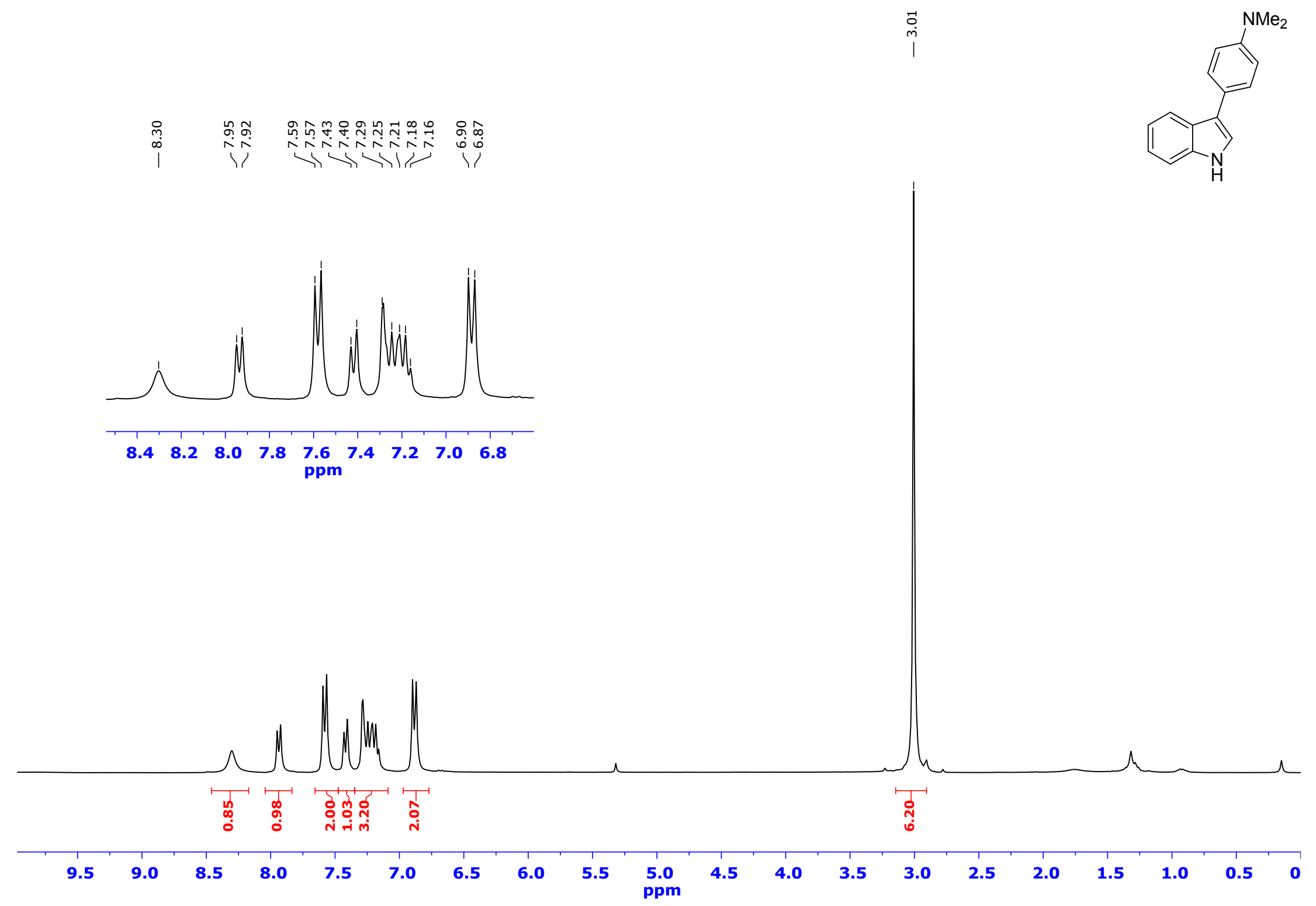

Figure S14: ${ }^{1} \mathrm{H}$ NMR spectrum of $4-\left(1 \mathrm{H}\right.$-indol-3-yl)- $N, N$-dimethylaniline $\left(300.13 \mathrm{MHz}, \mathrm{CD}_{2} \mathrm{Cl}_{2}\right)$. 


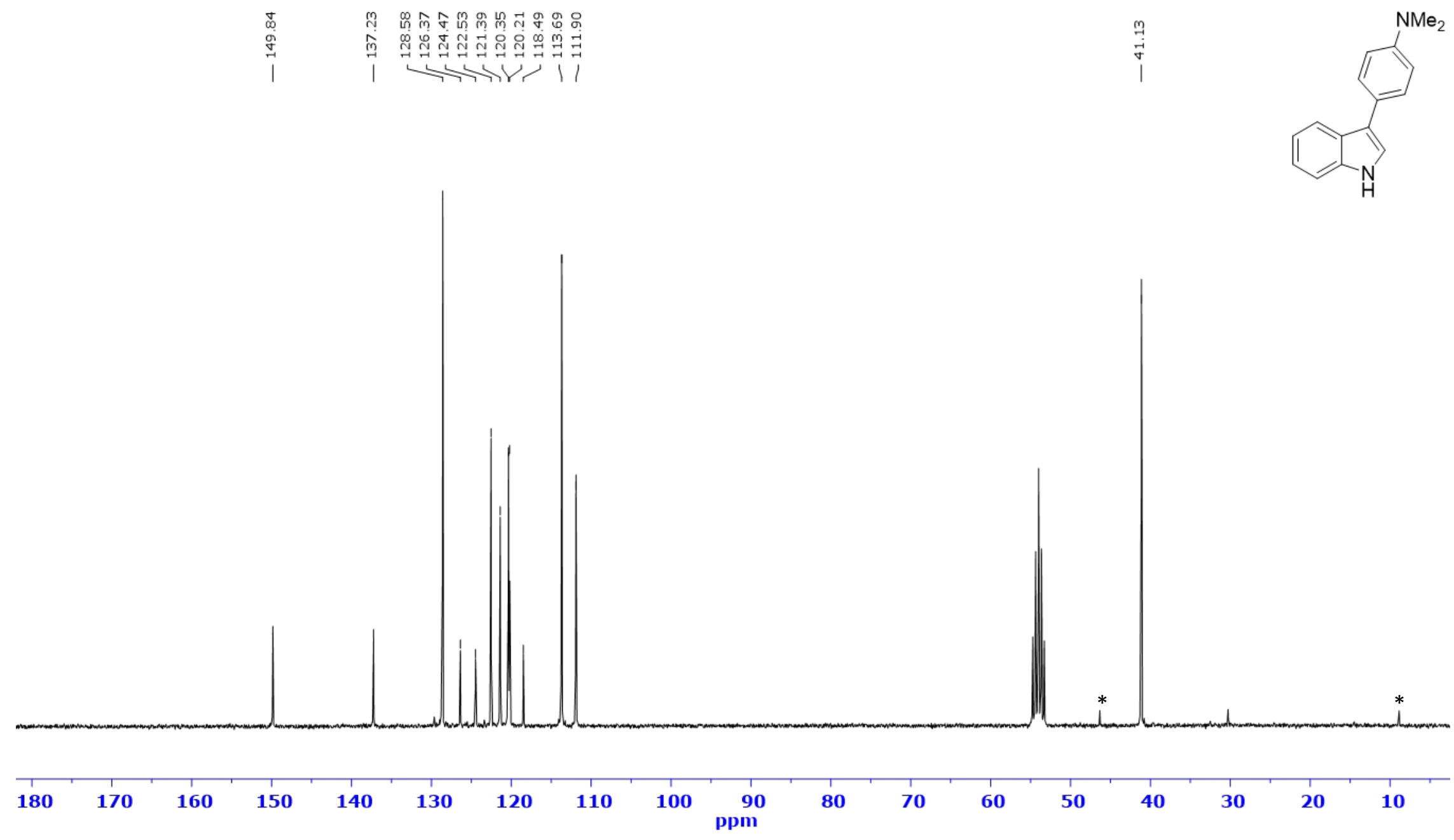

Figure S15: ${ }^{13} \mathrm{C}$ NMR spectrum of 4-(1H-indol-3-yl)- $\mathrm{N}, \mathrm{N}$-dimethylaniline $\left(75.47 \mathrm{MHz}, \mathrm{CD}_{2} \mathrm{Cl}_{2}\right)$. * Traces of triethylammonium salts from column chromatography. 


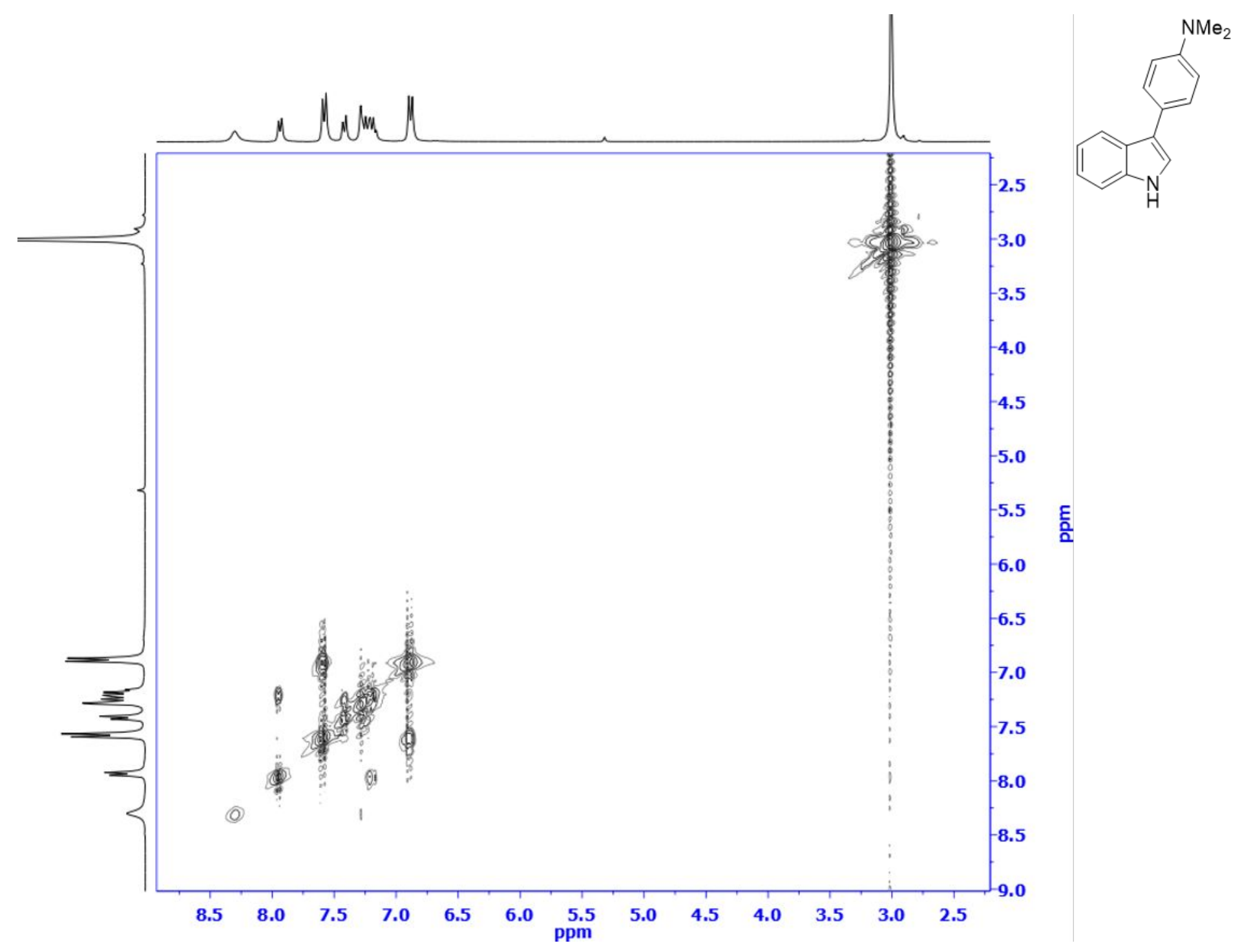

Figure S16: ${ }^{1} \mathrm{H}-{ }^{1} \mathrm{H}-\mathrm{COSY} N M R$ spectrum of $4-\left(1 \mathrm{H}\right.$-indol-3-yl)- $\mathrm{N}, \mathrm{N}$-dimethylaniline $\left(300.13 \mathrm{MHz}, \mathrm{CD}_{2} \mathrm{Cl}_{2}\right)$. 


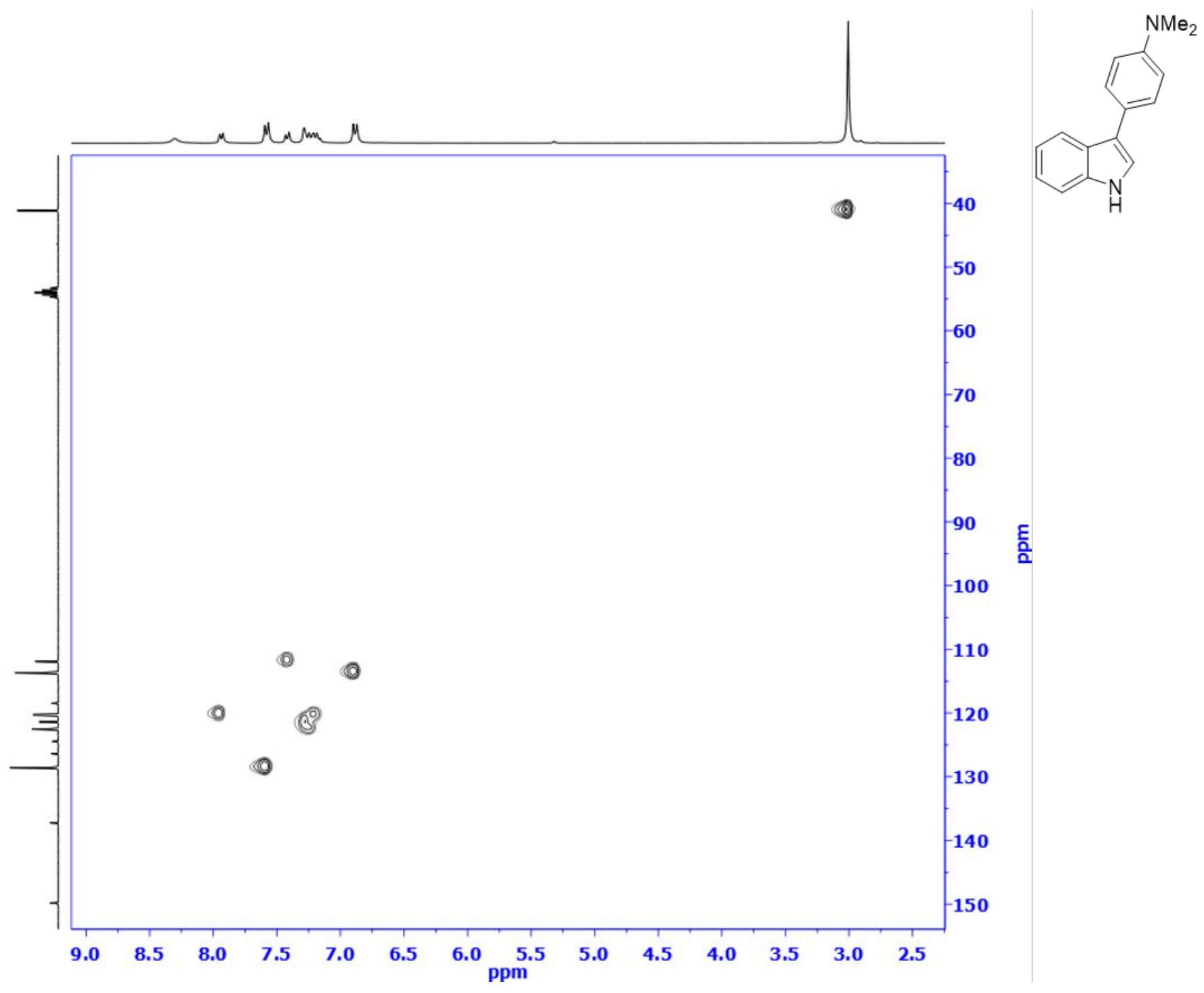

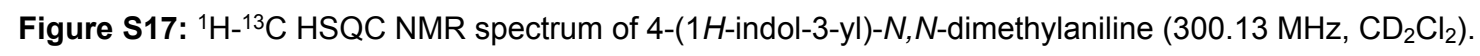




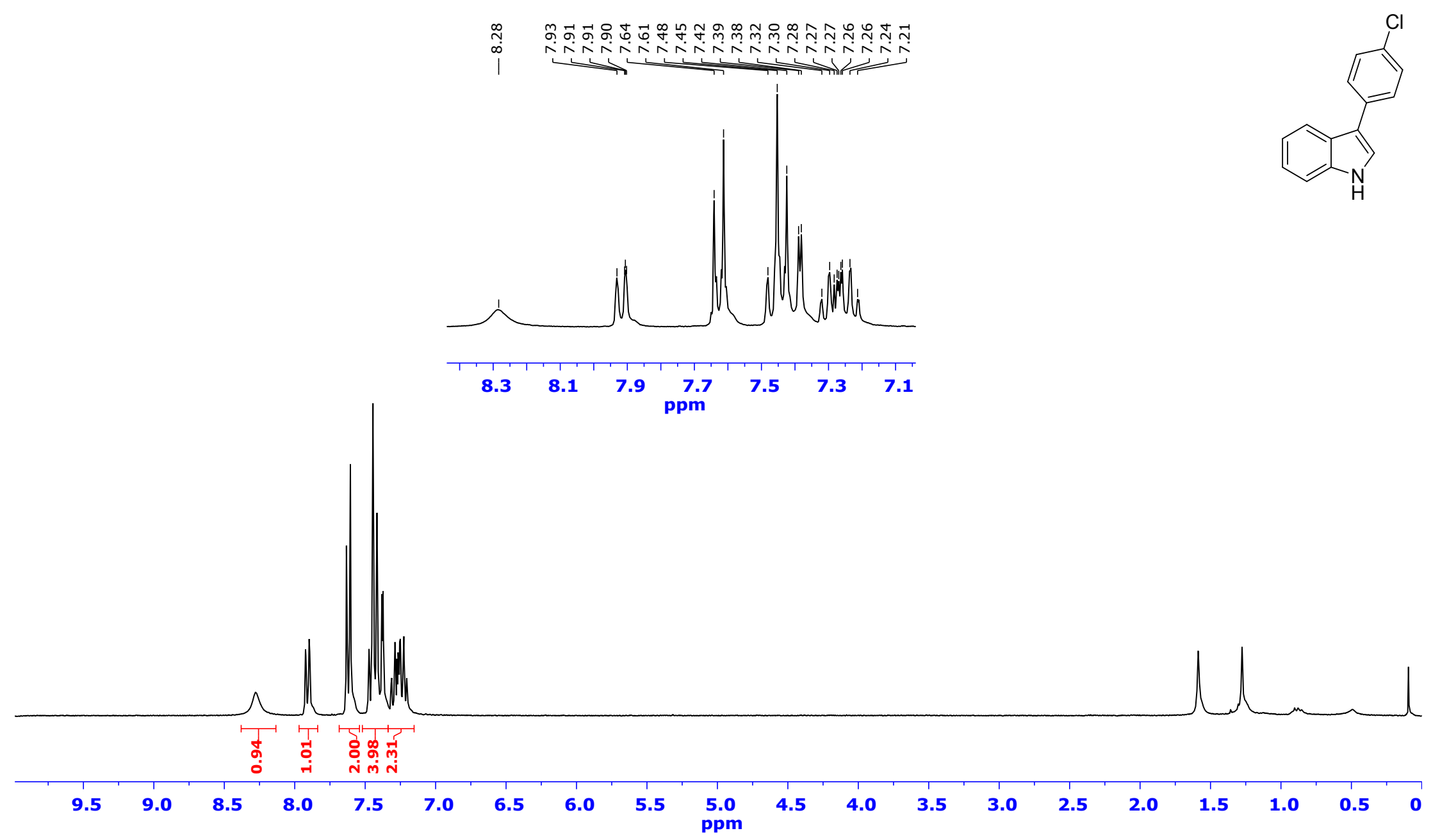

Figure S18: ${ }^{1} \mathrm{H}$ NMR spectrum of 3-(4-chlorophenyl)-1 $\mathrm{H}$-indole $\left(300.13 \mathrm{MHz}, \mathrm{CDCl}_{3}\right)$. 


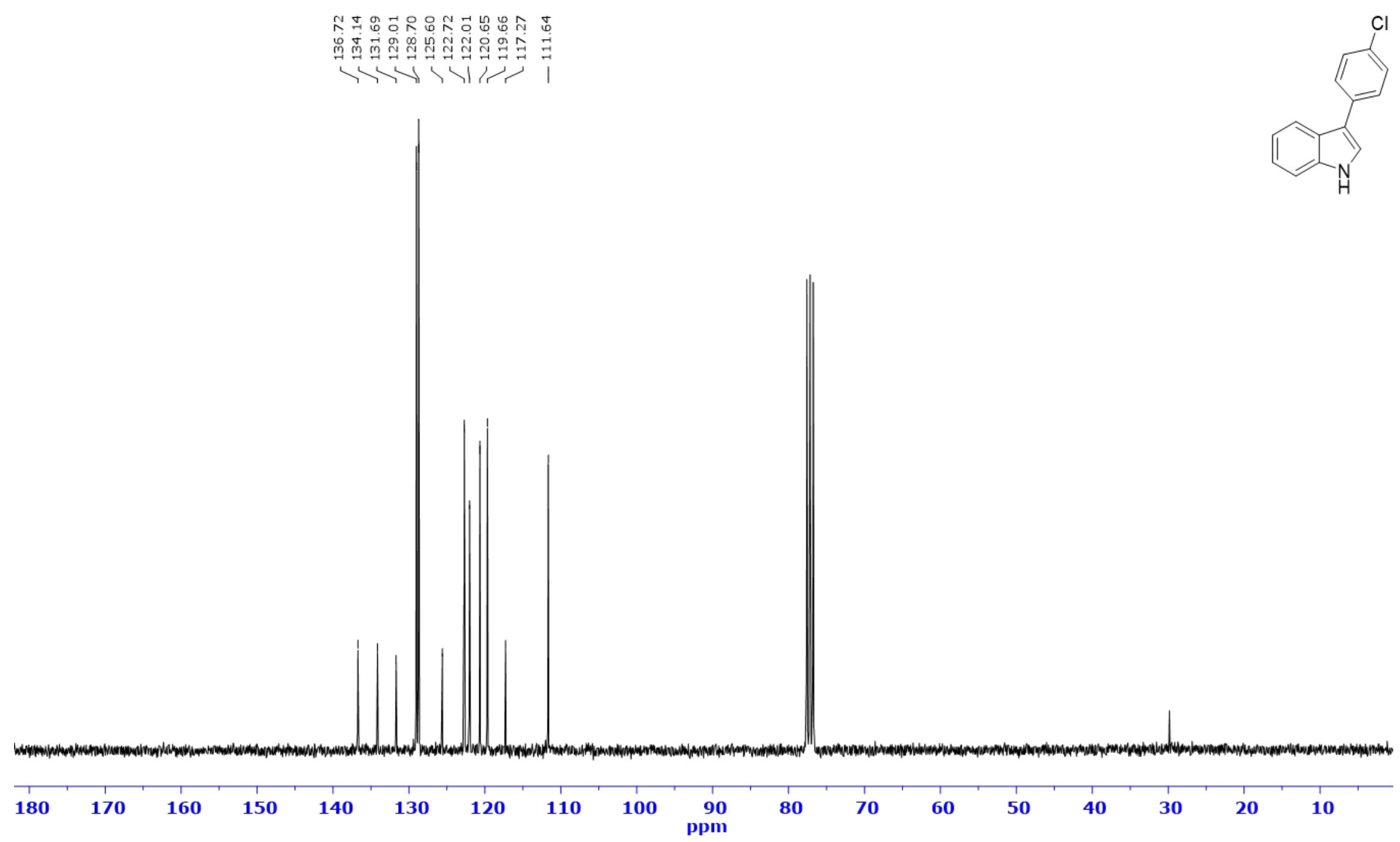

Figure S19: ${ }^{13} \mathrm{C}$ NMR spectrum of 3-(4-chlorophenyl)-1 H-indole $\left(75.47 \mathrm{MHz}, \mathrm{CDCl}_{3}\right)$. 

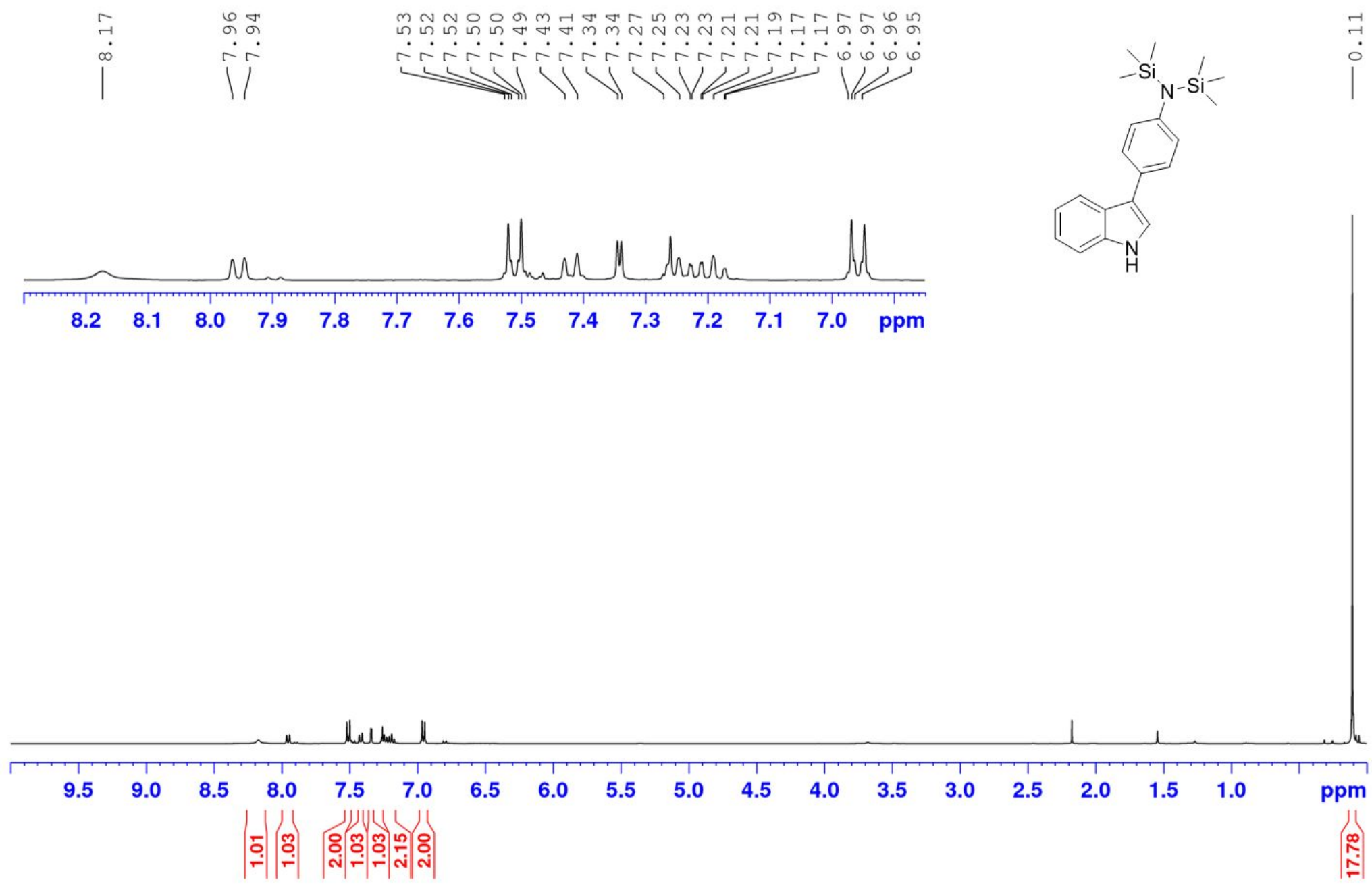

Figure S20: ${ }^{1} \mathrm{H}$ NMR spectrum of $\mathrm{N}$-(4-(1H-indol-3-yl)phenyl)-1,1,1-trimethyl- $\mathrm{N}$-(trimethylsilyl)silanamine $\left(400.13 \mathrm{MHz}, \mathrm{CDCl}_{3}\right)$. Note: after the purification a hydrolyzation of the TMS groups of a minor fraction of the product took place (final purity > 95\%). 


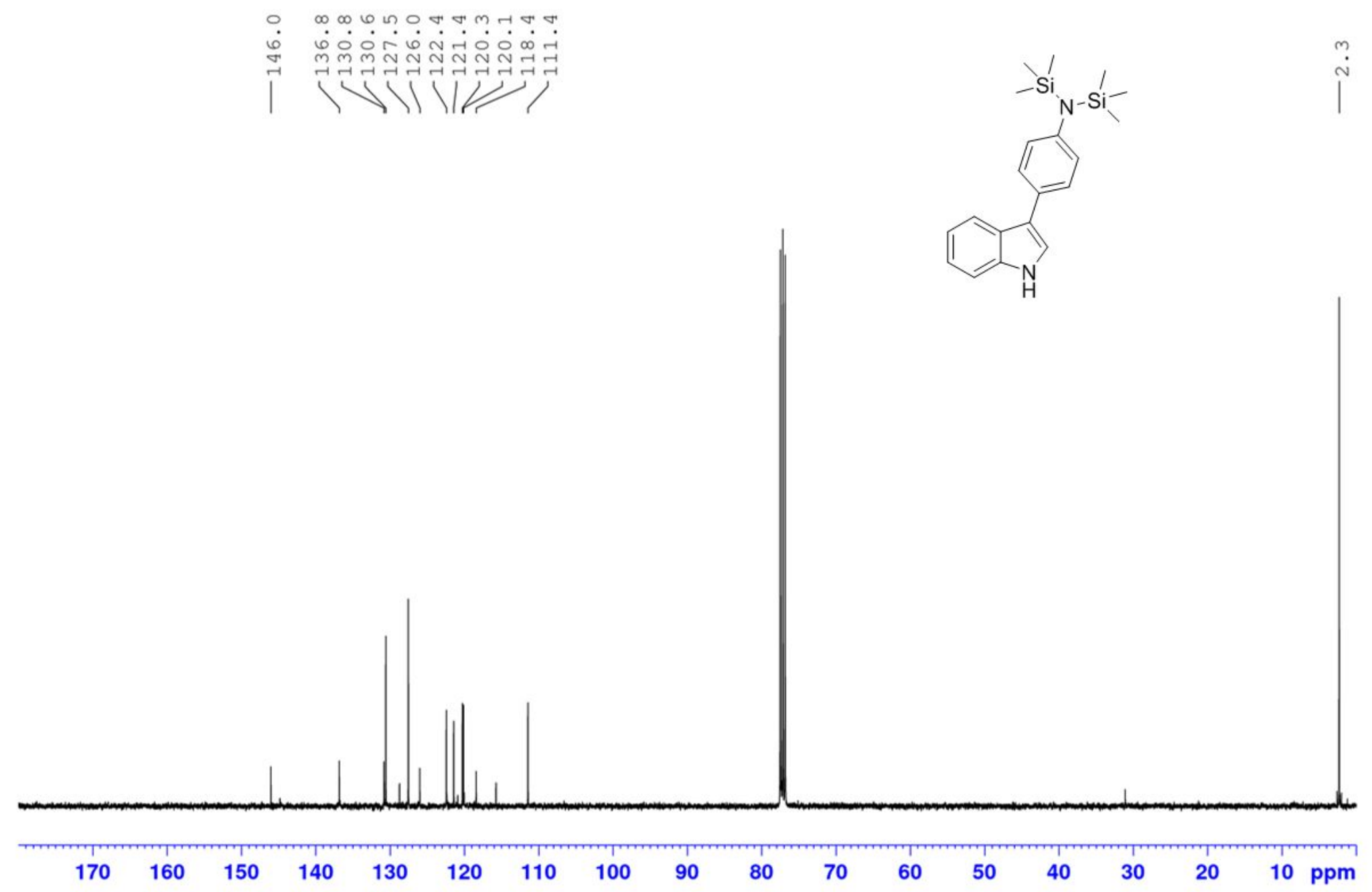

Figure S21: ${ }^{13} \mathrm{C}$ NMR spectrum of $\mathrm{N}$-(4-(1H-indol-3-yl)phenyl)-1,1,1-trimethyl- $\mathrm{N}$-(trimethylsilyl)silanamine $(100.61 \mathrm{MHz}, \mathrm{CDCl} 3) . \mathrm{Note}$ : after the purification a hydrolyzation of the TMS groups of a minor fraction of the product took place (final purity $>95 \%$ ). 


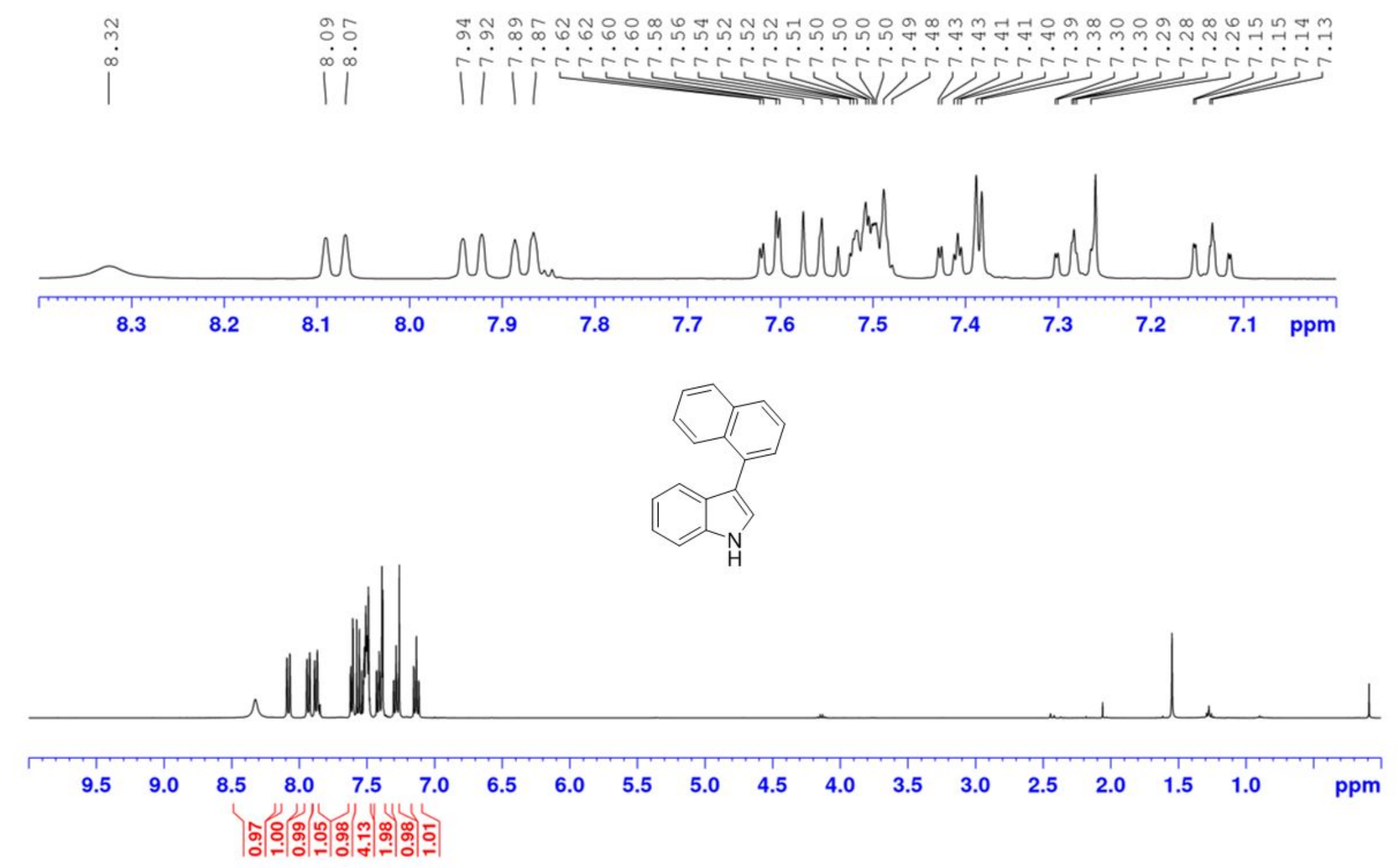

Figure S22: ${ }^{1} \mathrm{H}$ NMR spectrum of 3-(naphthalen-1-yl)-1 1 -indole $\left(400.13 \mathrm{MHz}, \mathrm{CDCl}_{3}\right)$. 


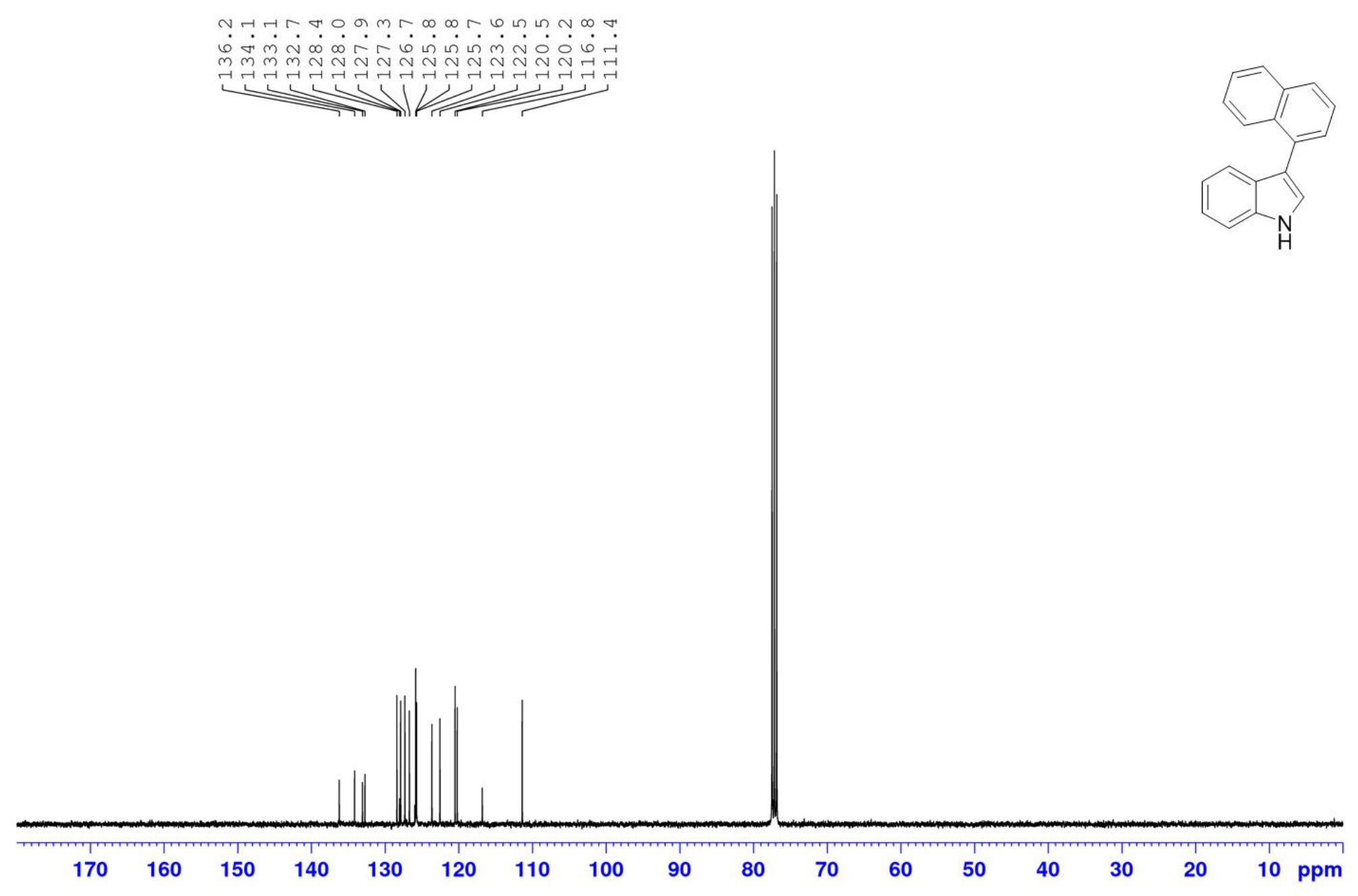

Figure S23: ${ }^{13} \mathrm{C}$ NMR spectrum of 3-(naphthalen-1-yl)-1H-indole $\left(100.61 \mathrm{MHz}, \mathrm{CDCl}_{3}\right)$. 

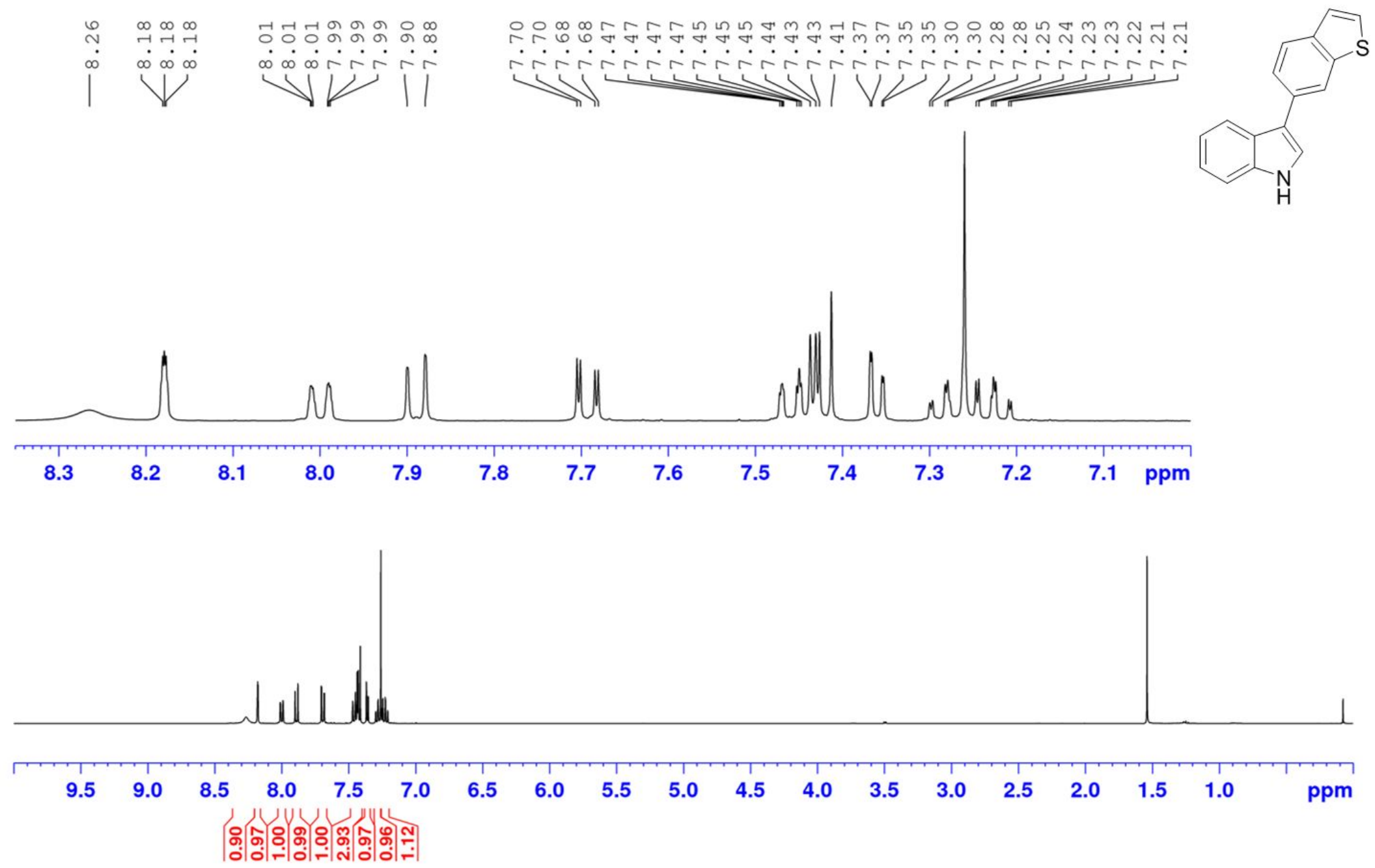

Figure S24: ${ }^{1} \mathrm{H}$ NMR spectrum of 3-(benzo[b]thiophen-6-yl)-1 $\mathrm{H}$-indole $\left(400.13 \mathrm{MHz}, \mathrm{CDCl}_{3}\right)$. 


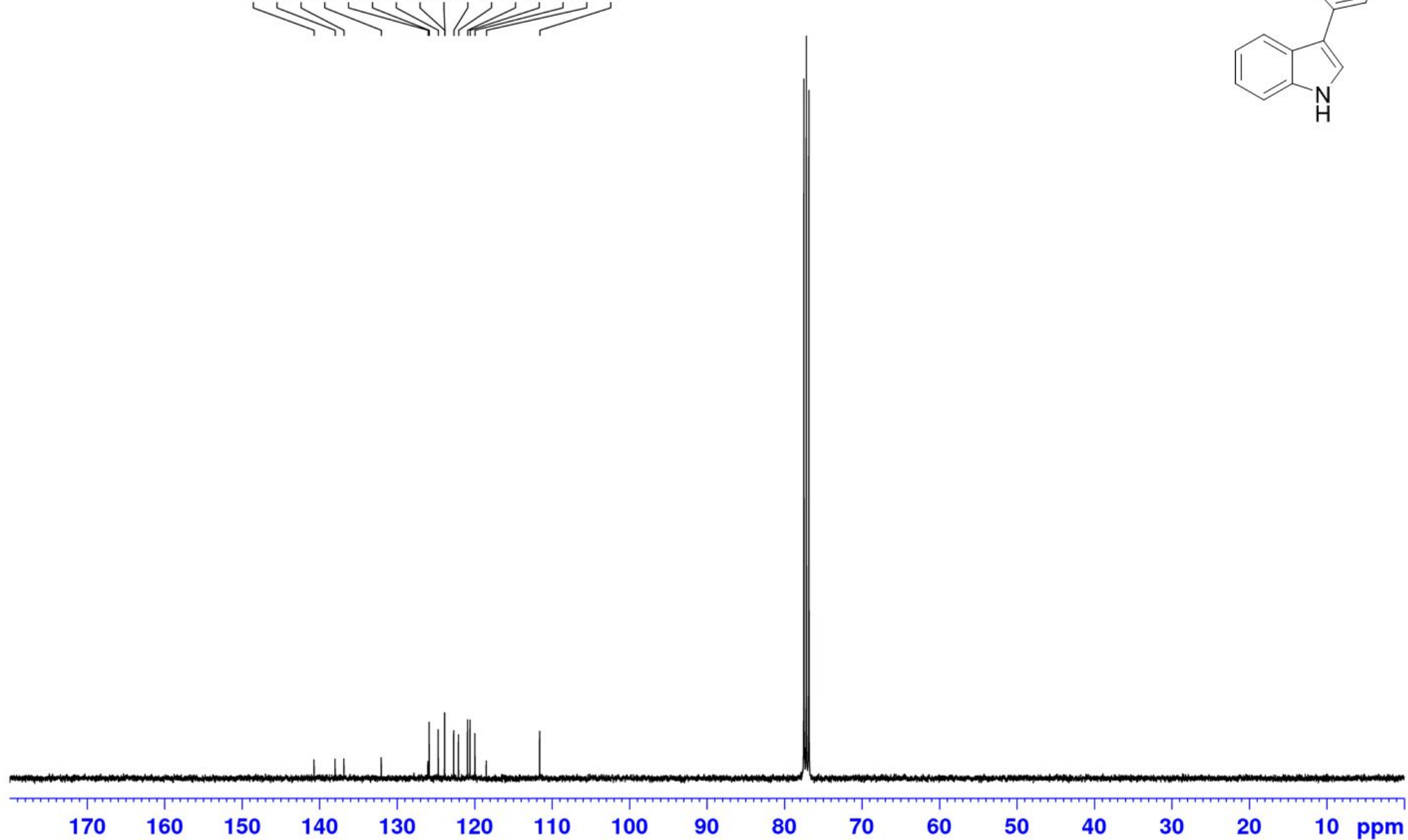

Figure S25: ${ }^{13} \mathrm{C}$ NMR spectrum of 3-(benzo[b]thiophen-6-yl)-1H-indole $\left(100.61 \mathrm{MHz}, \mathrm{CDCl}_{3}\right)$. 


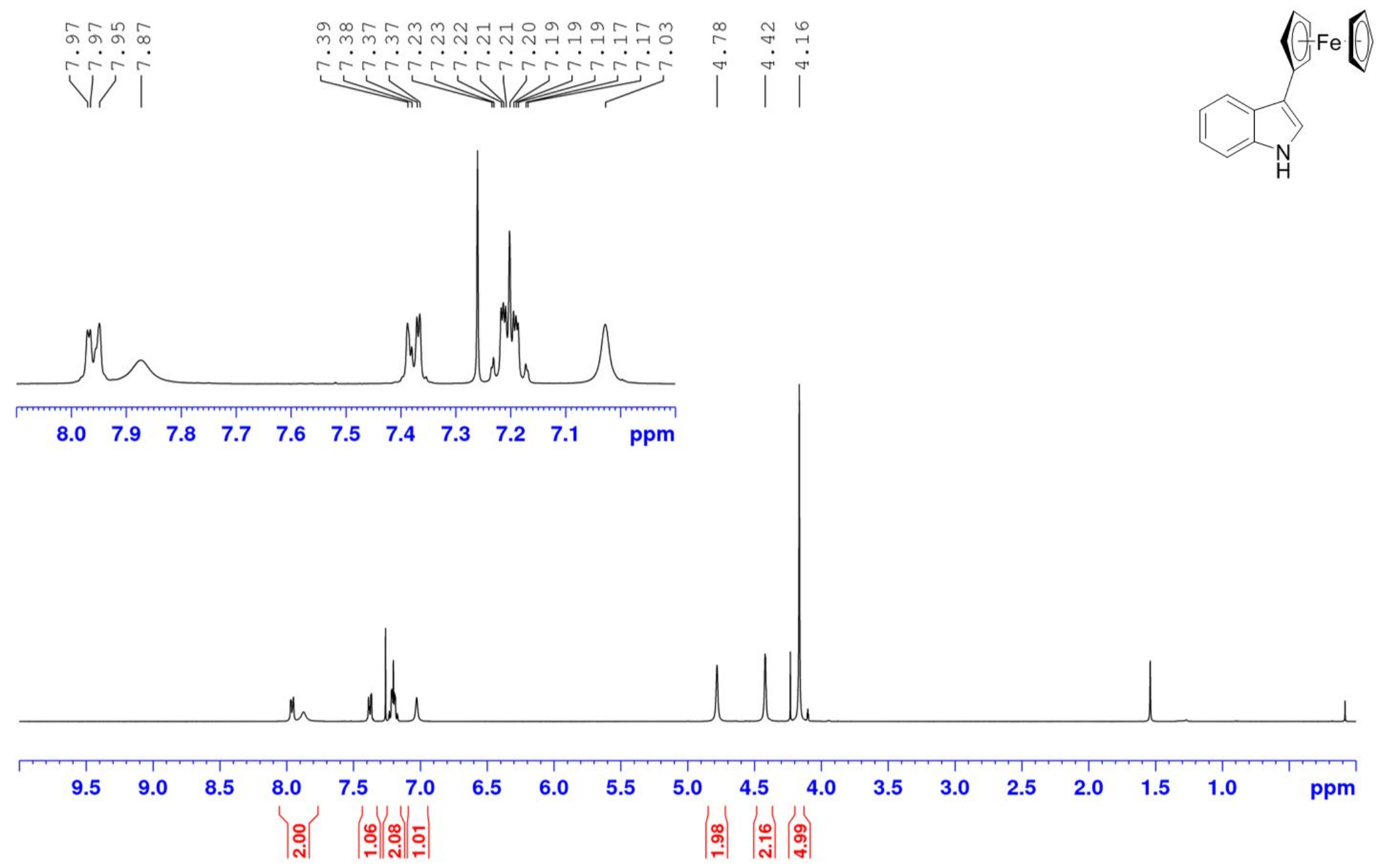

Figure S26: ${ }^{1} \mathrm{H}$ NMR spectrum of 3-ferrocenyl-1H-indole $\left(400.13 \mathrm{MHz}, \mathrm{CDCl}_{3}\right)$. Note: residual signals arising from the starting material (bromoferrocene) appear at $\delta: 4.23,4.10$ (product purity $>95 \%$ ). 


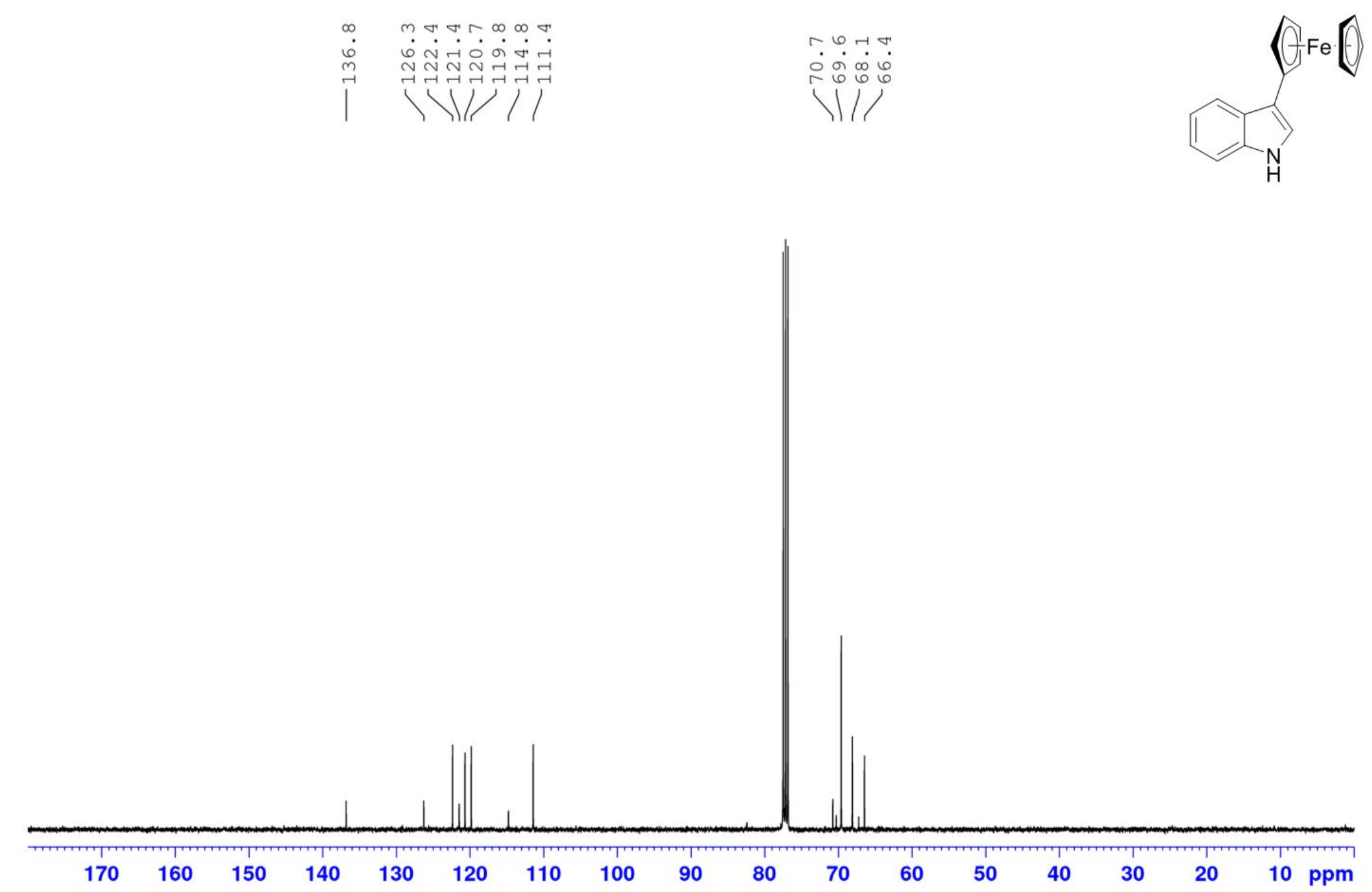

Figure S27: ${ }^{13} \mathrm{C}$ NMR spectrum of 3-ferrocenyl-1H-indole $\left(100.61 \mathrm{MHz}, \mathrm{CDCl}_{3}\right)$. Note: residual signals arising from the starting material (bromoferrocene) appear at $\delta: 70.3,67.2$ (product purity $>95 \%$ ). 

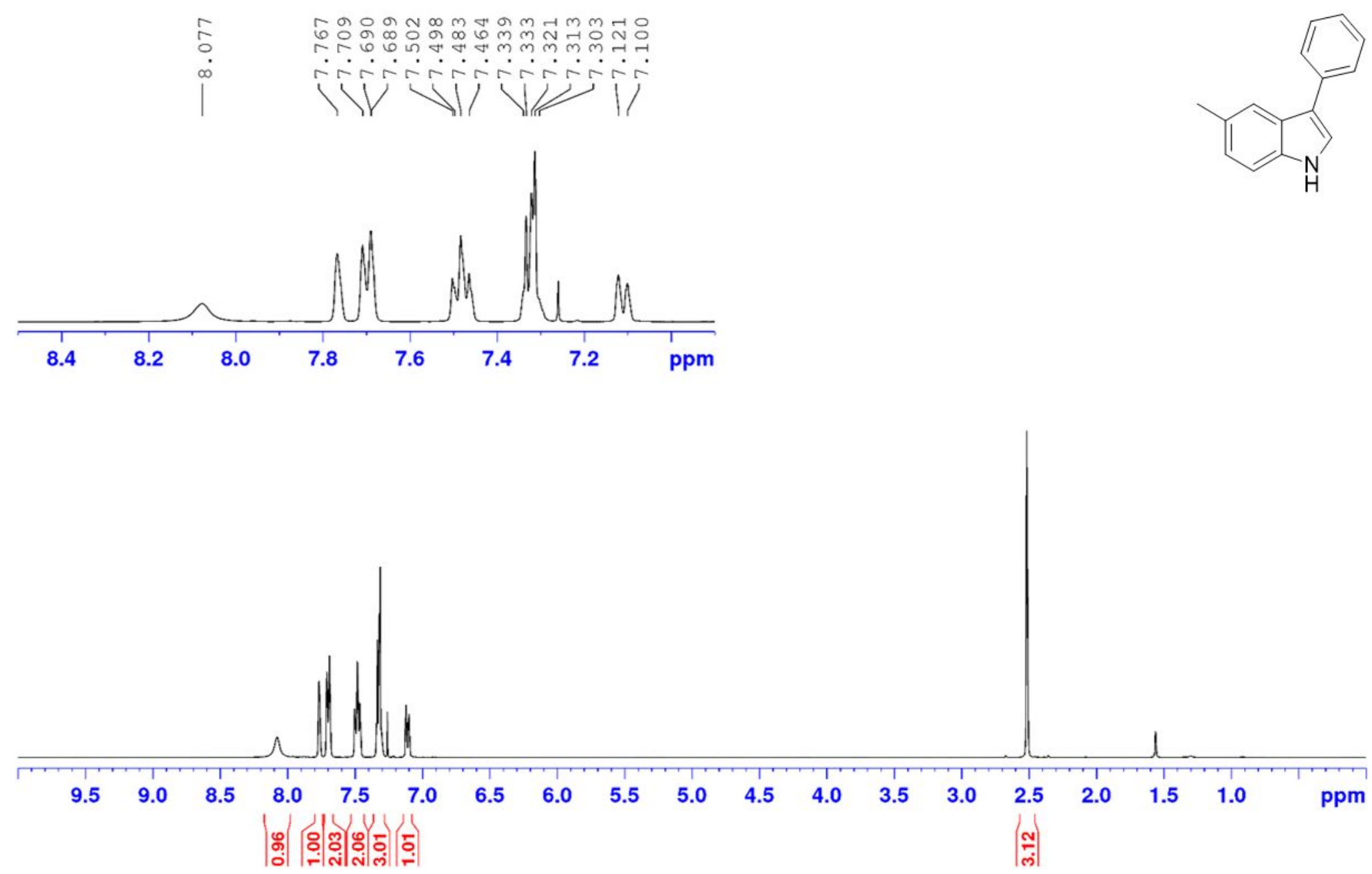

Figure S28: ${ }^{1} \mathrm{H}$ NMR spectrum of 5-methyl-3-phenyl-1H-indole $\left(400.13 \mathrm{MHz}, \mathrm{CDCl}_{3}\right)$. 

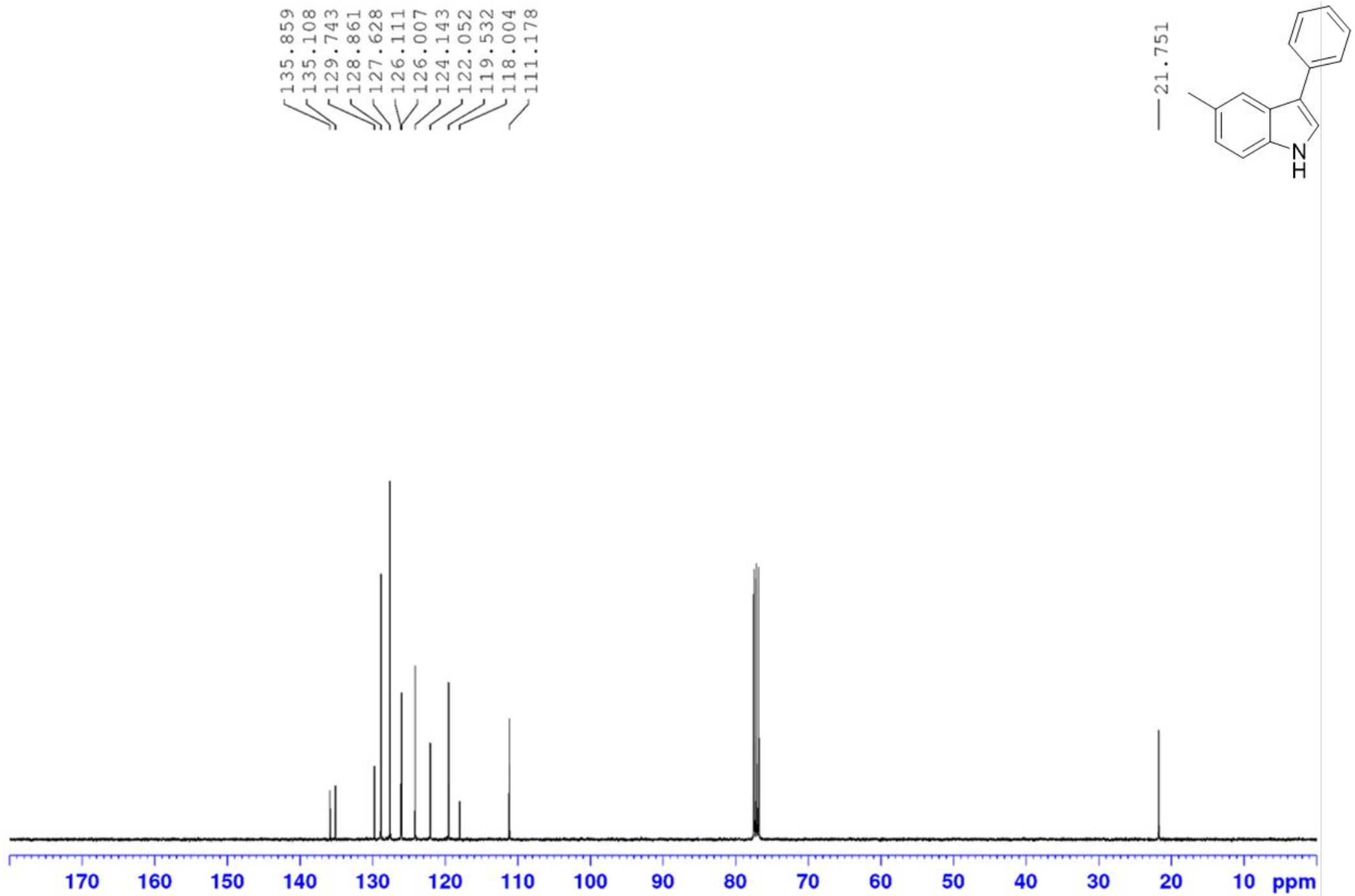

Figure S29: ${ }^{13} \mathrm{C}$ NMR spectrum of 5-methyl-3-phenyl- $1 \mathrm{H}$-indole $\left(100.61 \mathrm{MHz}, \mathrm{CDCl}_{3}\right)$. 


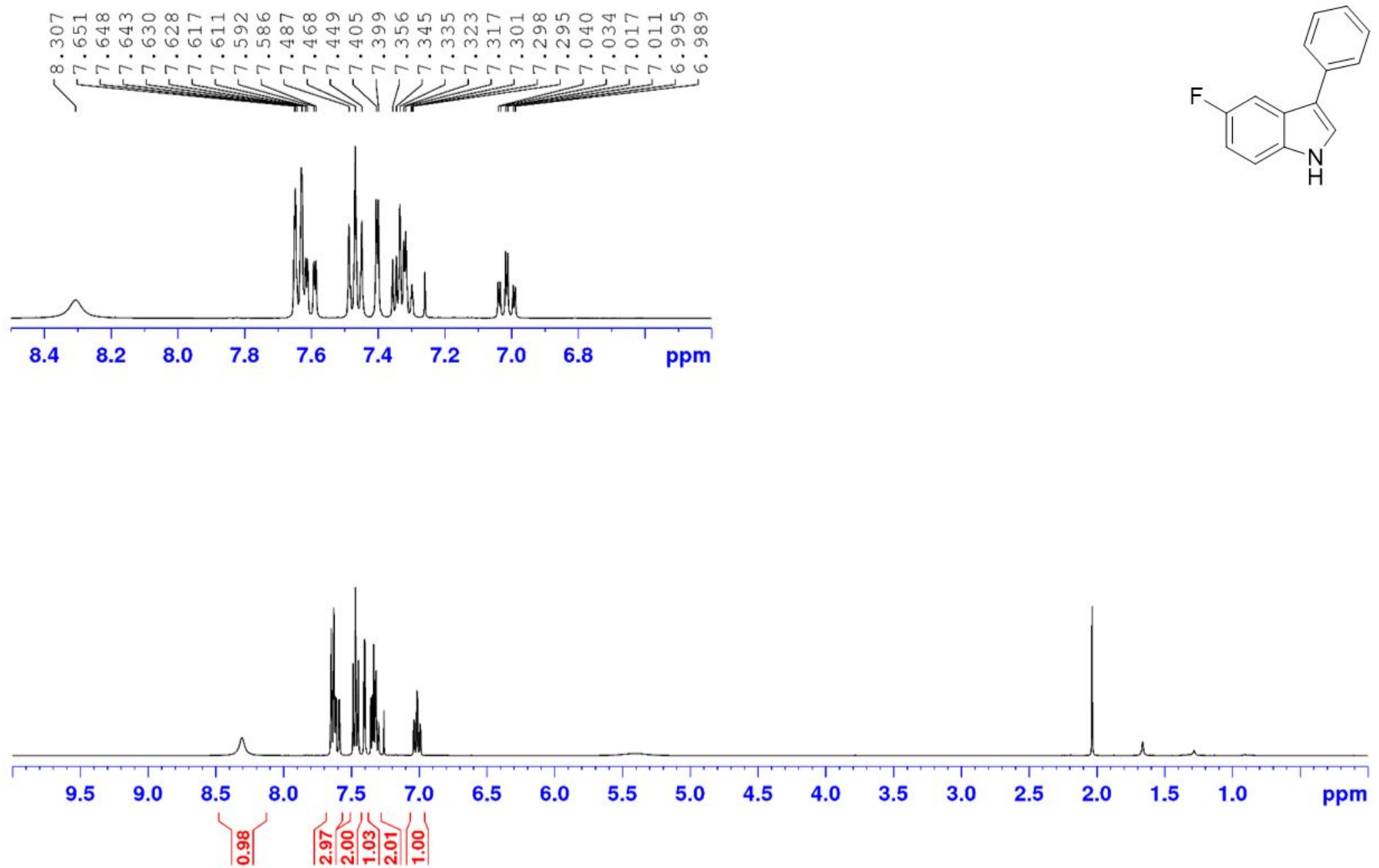

Figure S30: ${ }^{1} \mathrm{H}$ NMR spectrum of 5-fluoro-3-phenyl-1H-indole $\left(400.13 \mathrm{MHz}, \mathrm{CDCl}_{3}\right)$. 

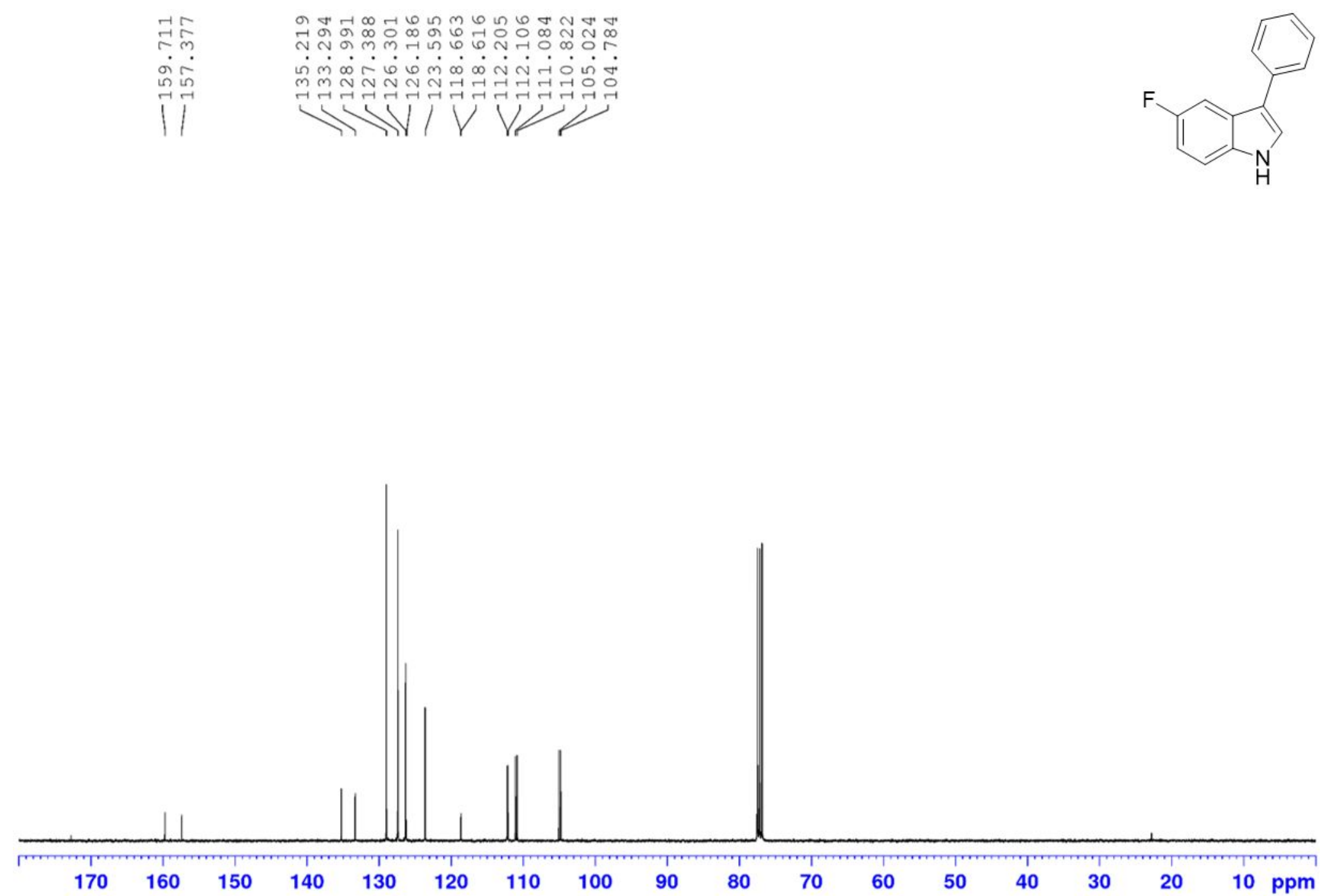

Figure S31: ${ }^{13} \mathrm{C}$ NMR spectrum of 5-fluoro-3-phenyl-1 $\mathrm{H}$-indole $\left(100.61 \mathrm{MHz}, \mathrm{CDCl}_{3}\right)$. 

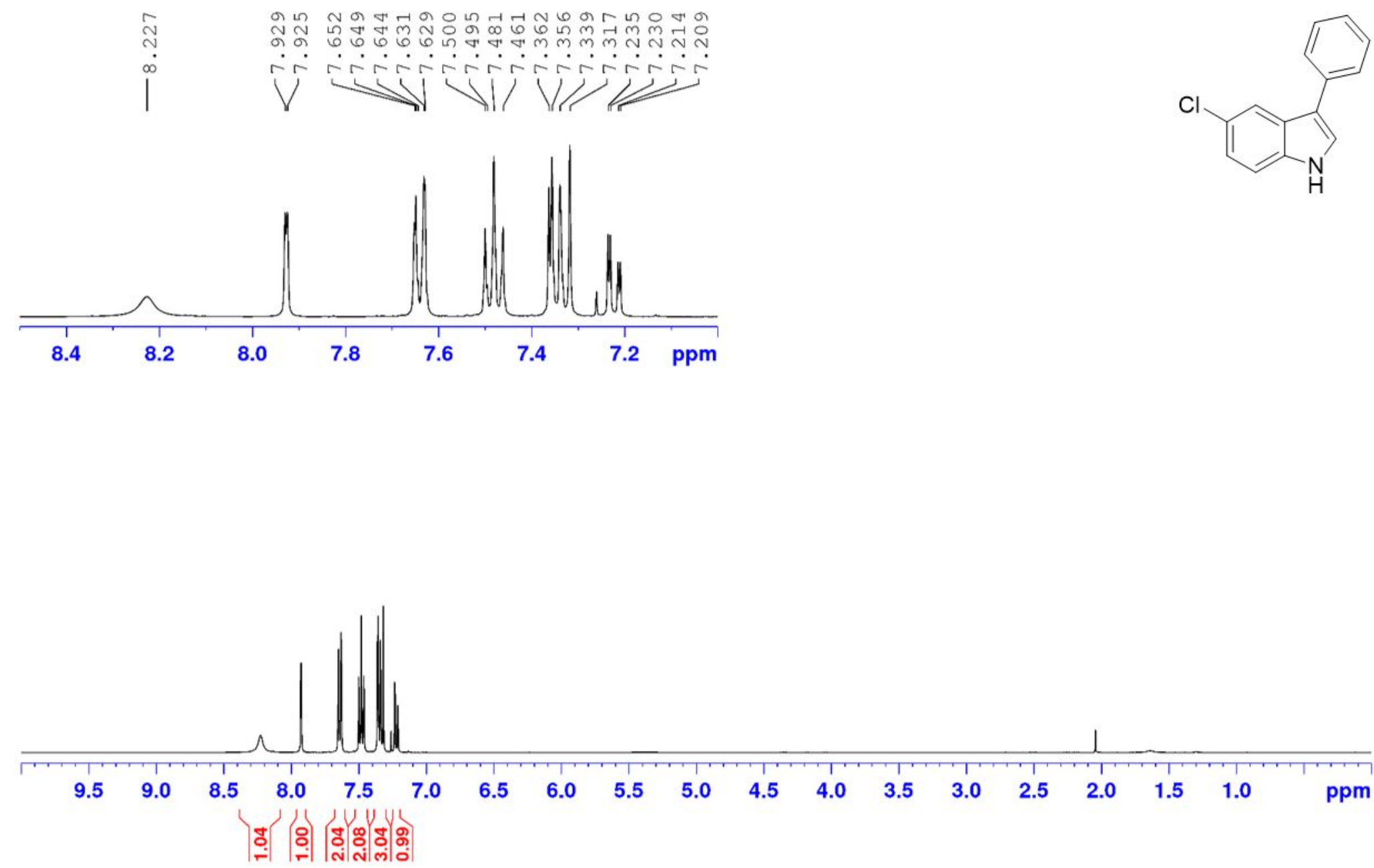

Figure S32: ${ }^{1} \mathrm{H}$ NMR spectrum of 5-chloro-3-phenyl-1 $\mathrm{H}$-indole $\left(400.13 \mathrm{MHz}, \mathrm{CDCl}_{3}\right)$. 

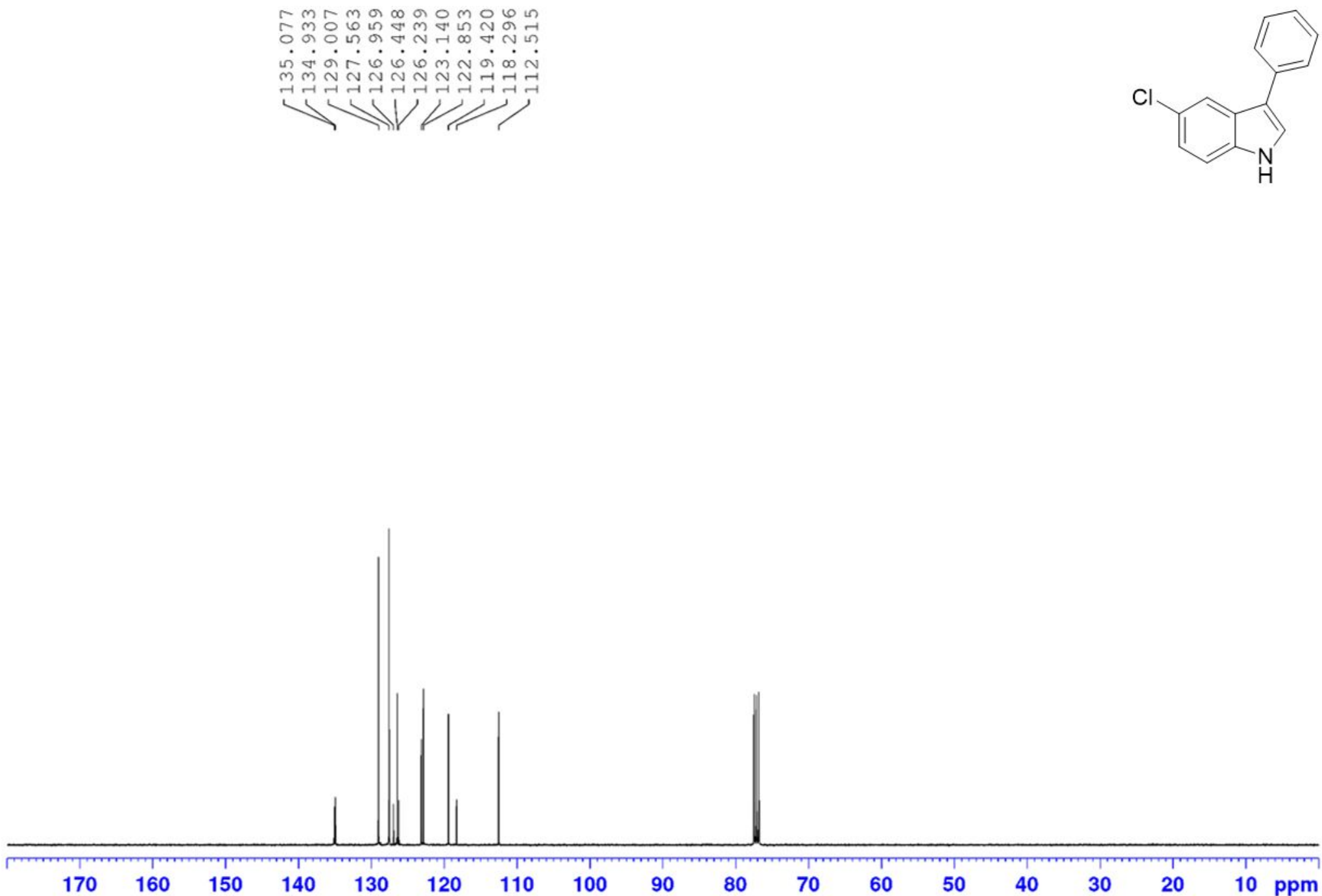

Figure S33: ${ }^{13} \mathrm{C}$ NMR spectrum of 5-chloro-3-phenyl-1H-indole $\left(100.61 \mathrm{MHz}, \mathrm{CDCl}_{3}\right)$. 

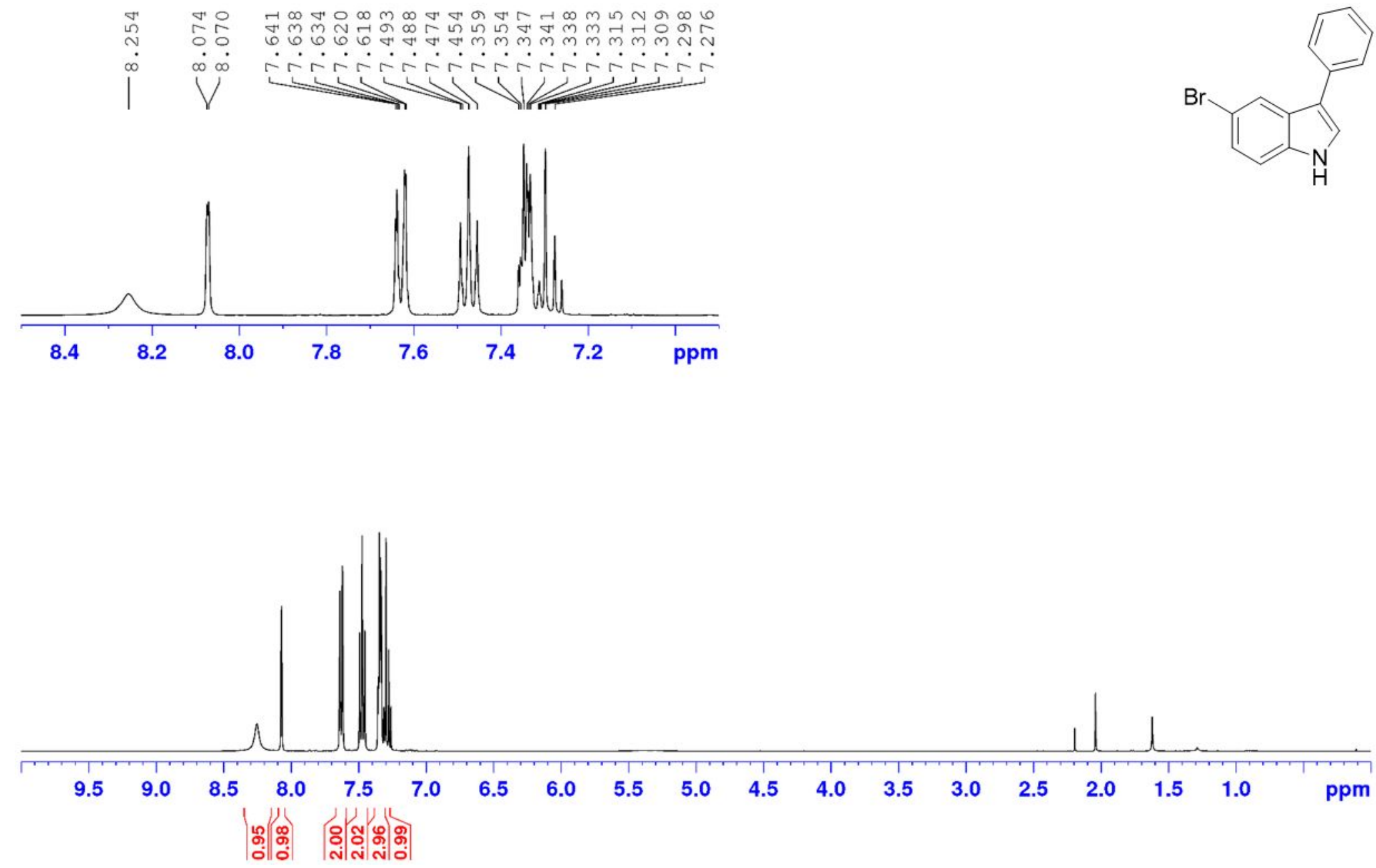

Figure S34: ${ }^{1} \mathrm{H}$ NMR spectrum of 5-bromo-3-phenyl-1H-indole $\left(400.13 \mathrm{MHz}, \mathrm{CDCl}_{3}\right)$. 

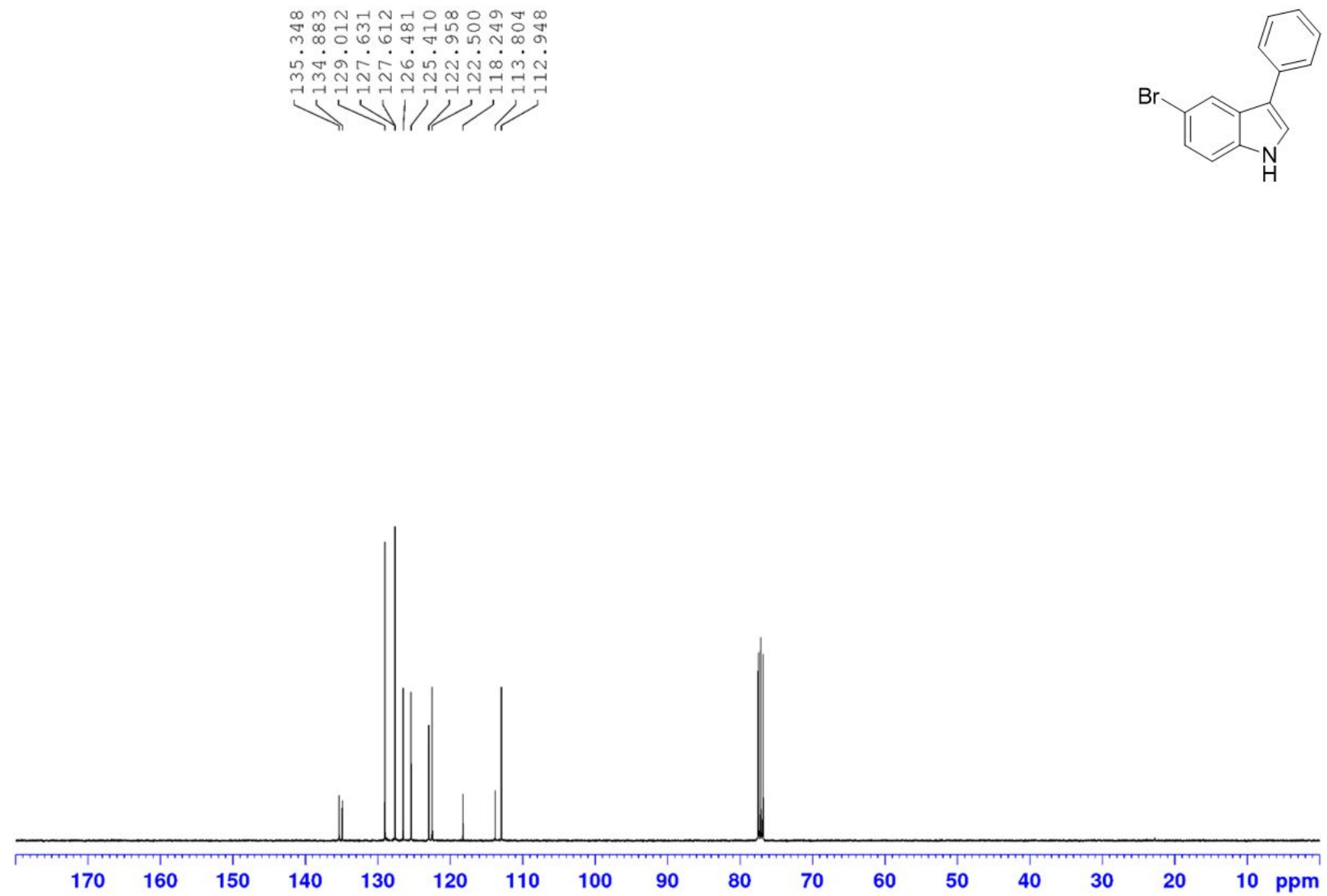

Figure S35: ${ }^{13} \mathrm{C}$ NMR spectrum of 5-bromo-3-phenyl-1H-indole (100.61 MHz, $\left.\mathrm{CDCl}_{3}\right)$. 

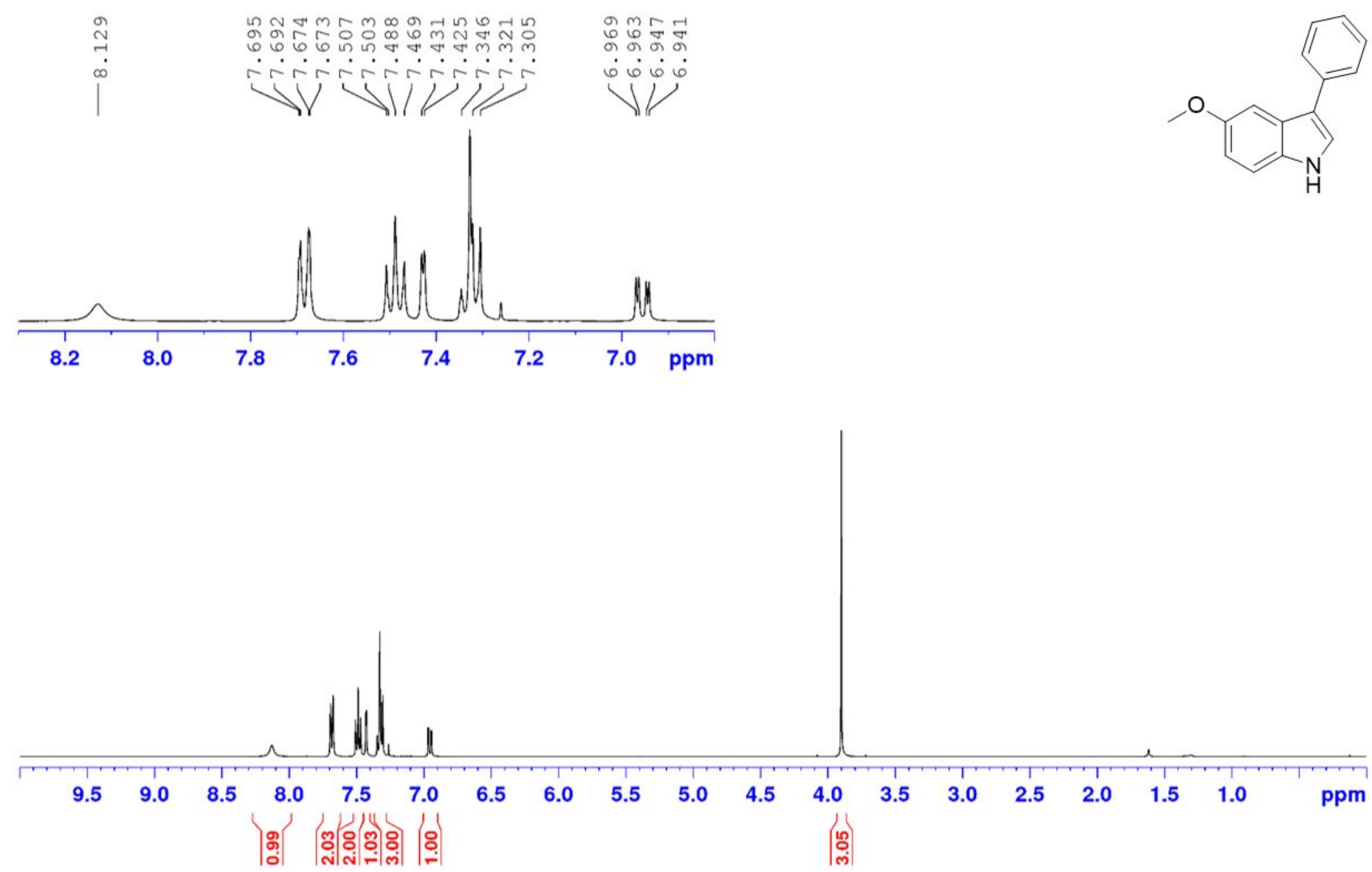

Figure S36: ${ }^{1} \mathrm{H}$ NMR spectrum of 5-methoxy-3-phenyl-1 $\mathrm{H}$-indole $\left(400.13 \mathrm{MHz}, \mathrm{CDCl}_{3}\right)$. 

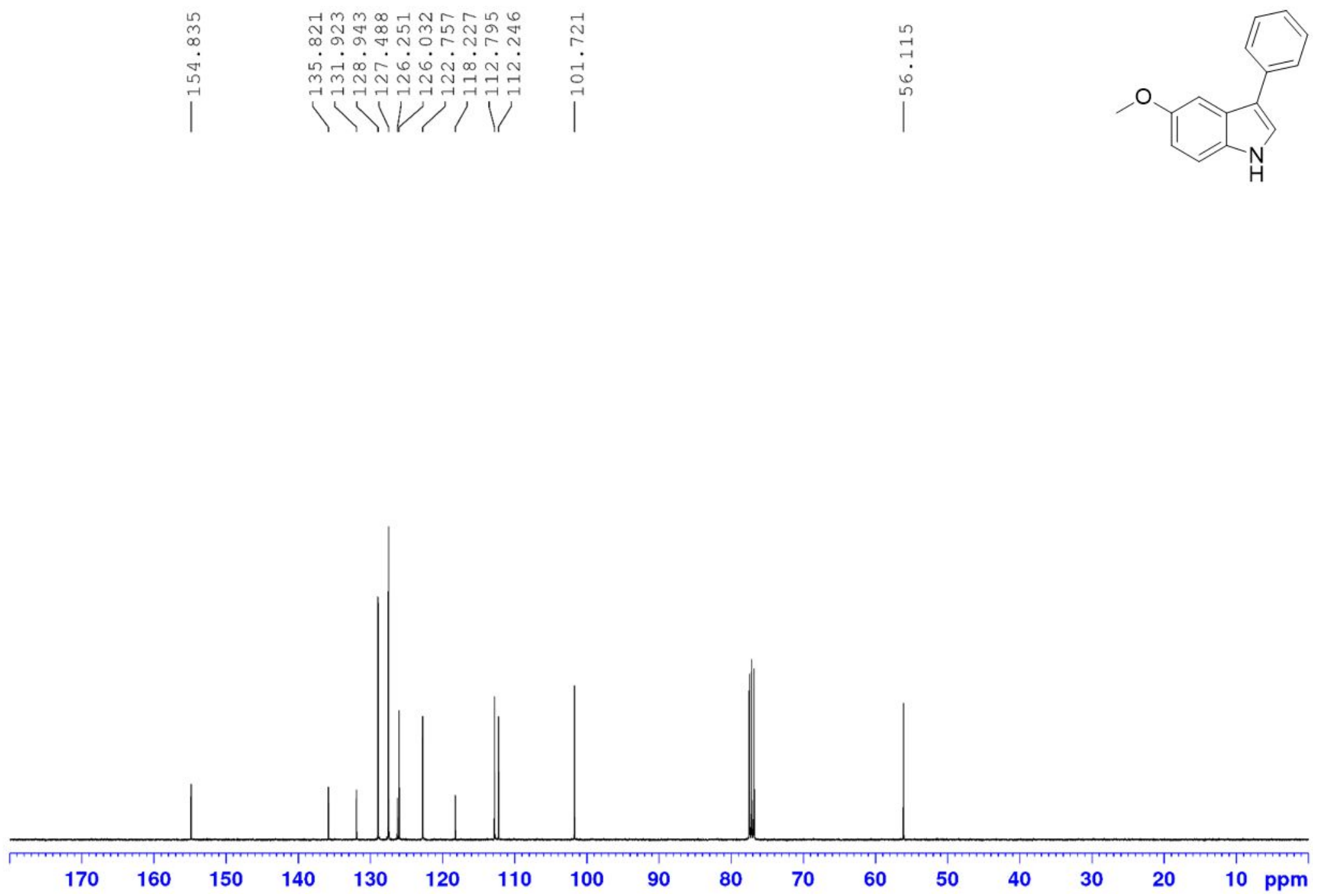

Figure S37: ${ }^{13} \mathrm{C}$ NMR spectrum of 5-methoxy-3-phenyl-1H-indole $\left(100.61 \mathrm{MHz}, \mathrm{CDCl}_{3}\right)$. 

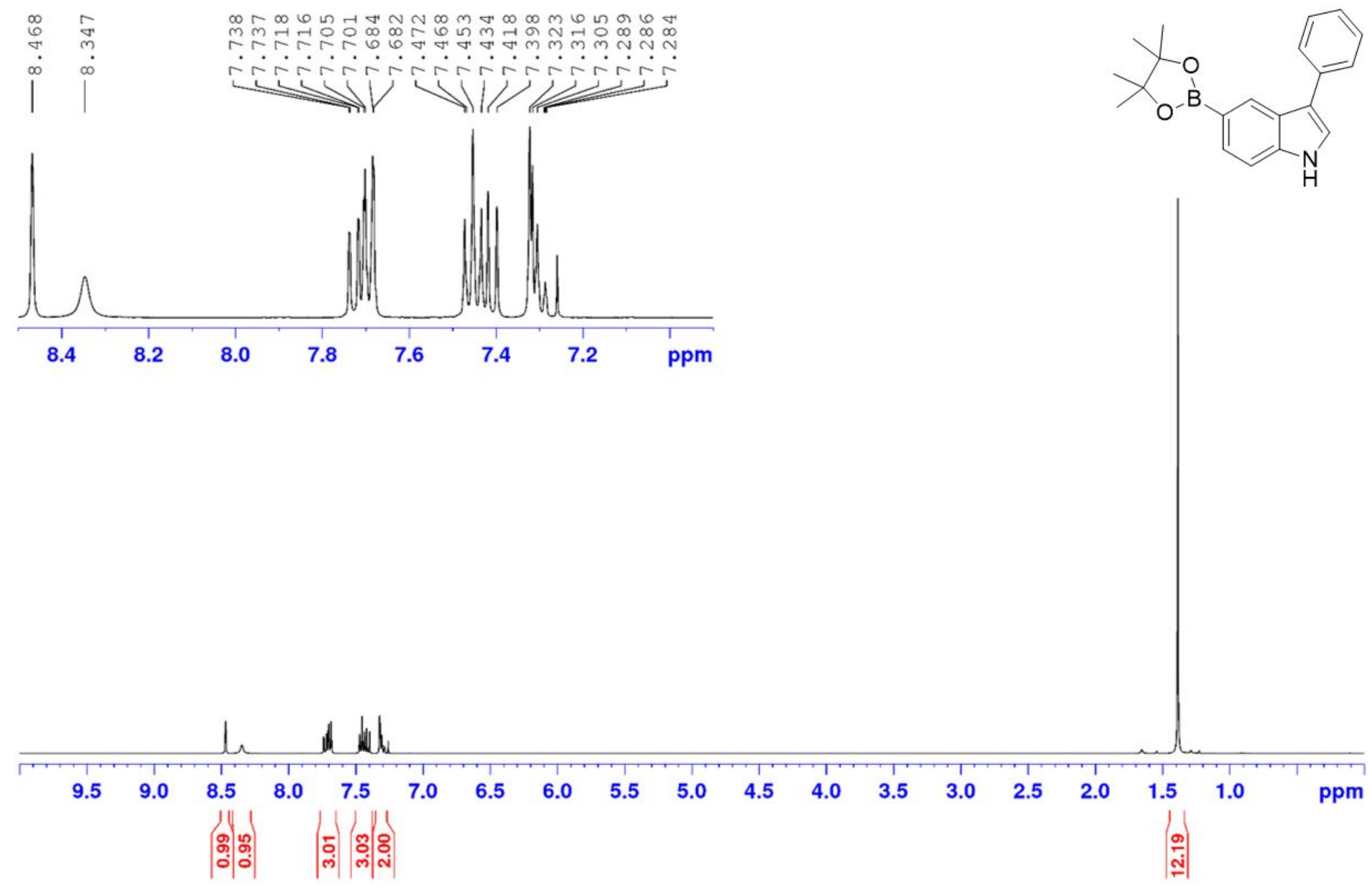

Figure S38: ${ }^{1} \mathrm{H}$ NMR spectrum of 3-phenyl-5-(4,4,5,5-tetramethyl-1,3,2-dioxaborolan-2-yl)-1H-indole $\left(400.13 \mathrm{MHz} \mathrm{CDCl}_{3}\right)$. 

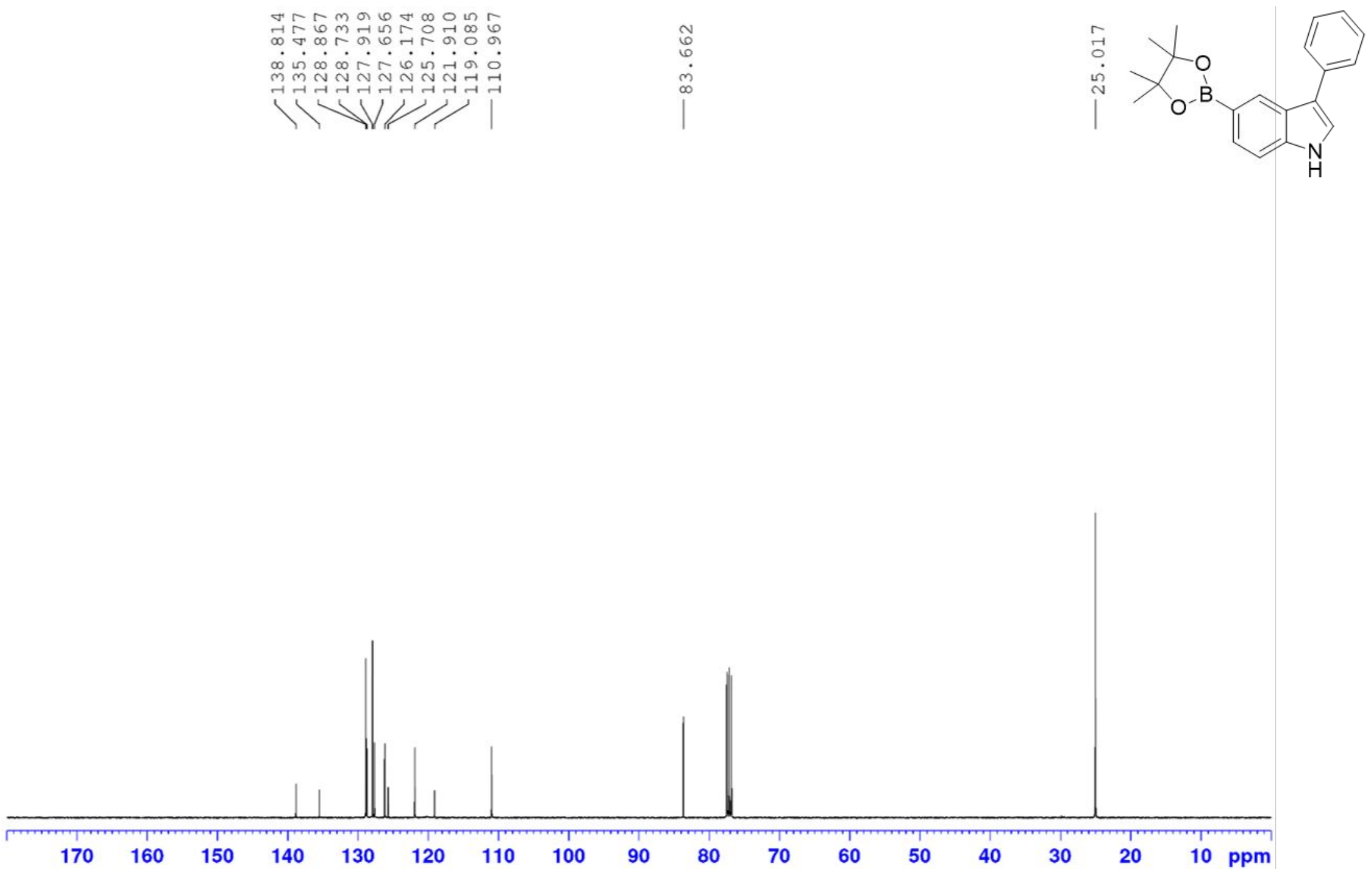

Figure S39: ${ }^{13} \mathrm{C}$ NMR spectrum of 3-phenyl-5-(4,4,5,5-tetramethyl-1,3,2-dioxaborolan-2-yl)-1H-indole (100.61 MHz, $\left.\mathrm{CDCl}_{3}\right)$. 


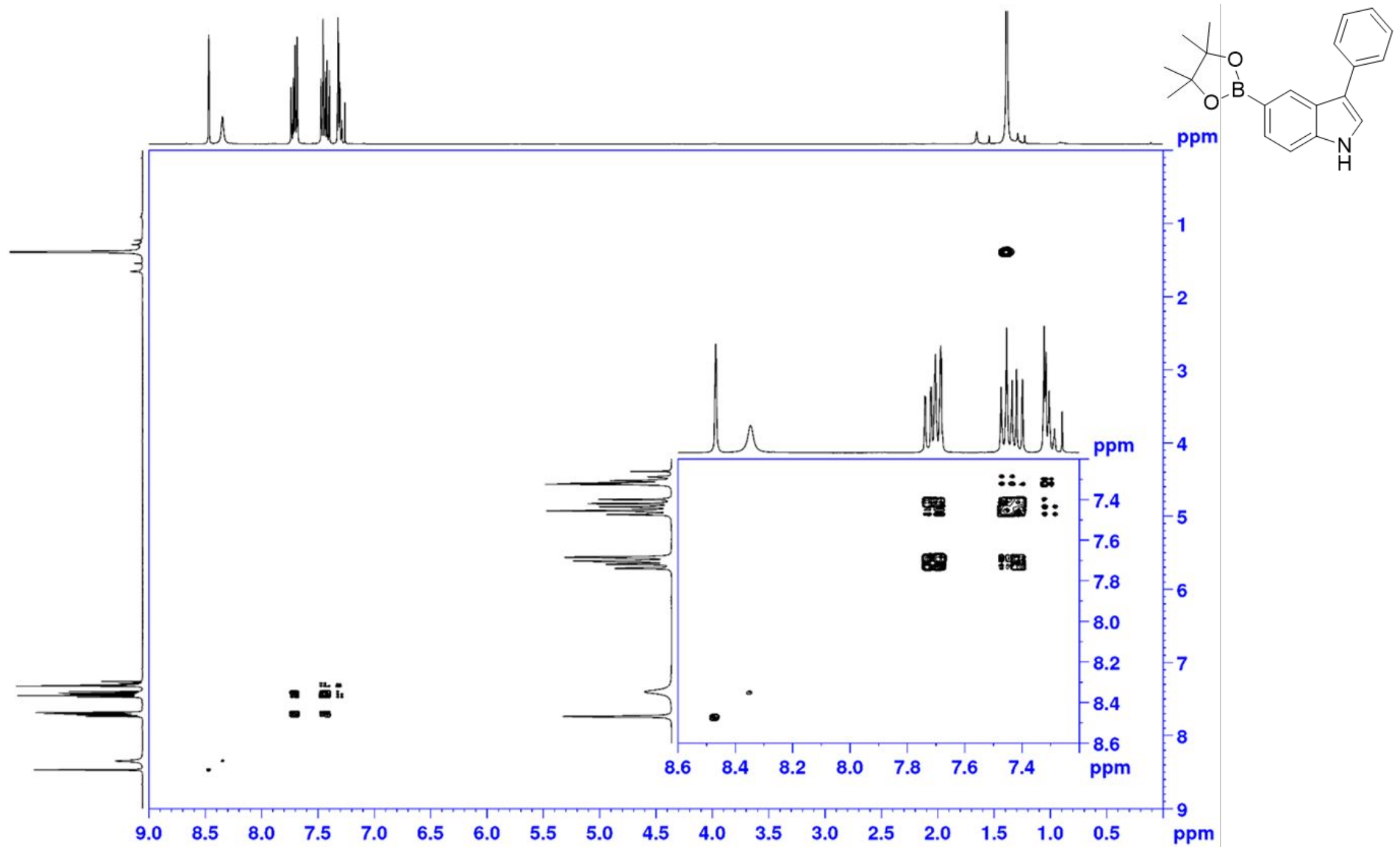

Figure S40: ${ }^{1} \mathrm{H}-{ }^{1} \mathrm{H}$ COSY NMR spectrum of 3-phenyl-5-(4,4,5,5-tetramethyl-1,3,2-dioxaborolan-2-yl)-1 $\mathrm{H}$-indole $\left(400.13 \mathrm{MHz}, \mathrm{CDCl}_{3}\right)$ 


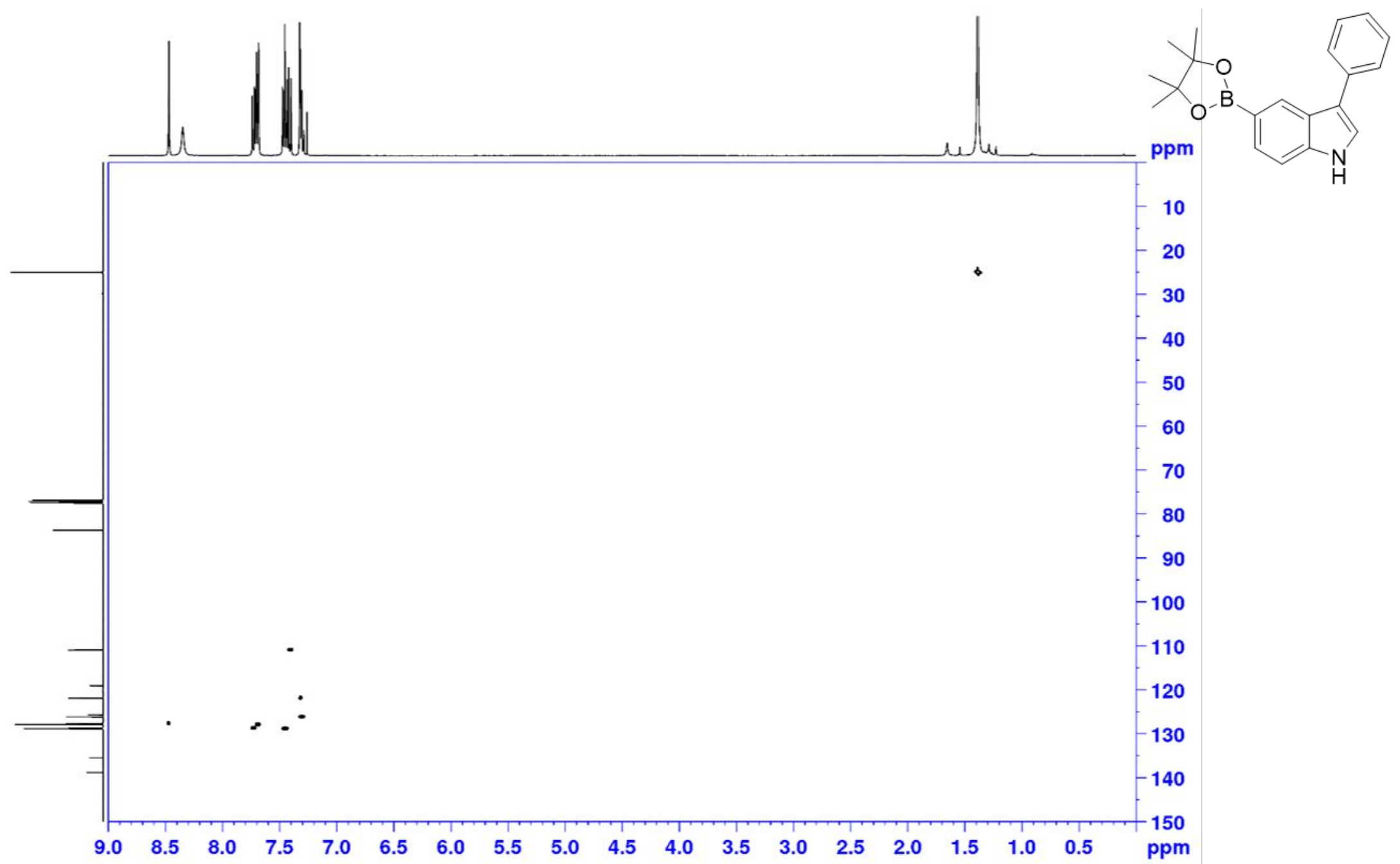

Figure S41: ${ }^{1} \mathrm{H}-{ }^{13} \mathrm{C}$ HSQC NMR spectrum of 3-phenyl-5-(4,4,5,5-tetramethyl-1,3,2-dioxaborolan-2-yl)-1 H-indole $\left(400.13 \mathrm{MHz}, \mathrm{CDCl}_{3}\right)$. 


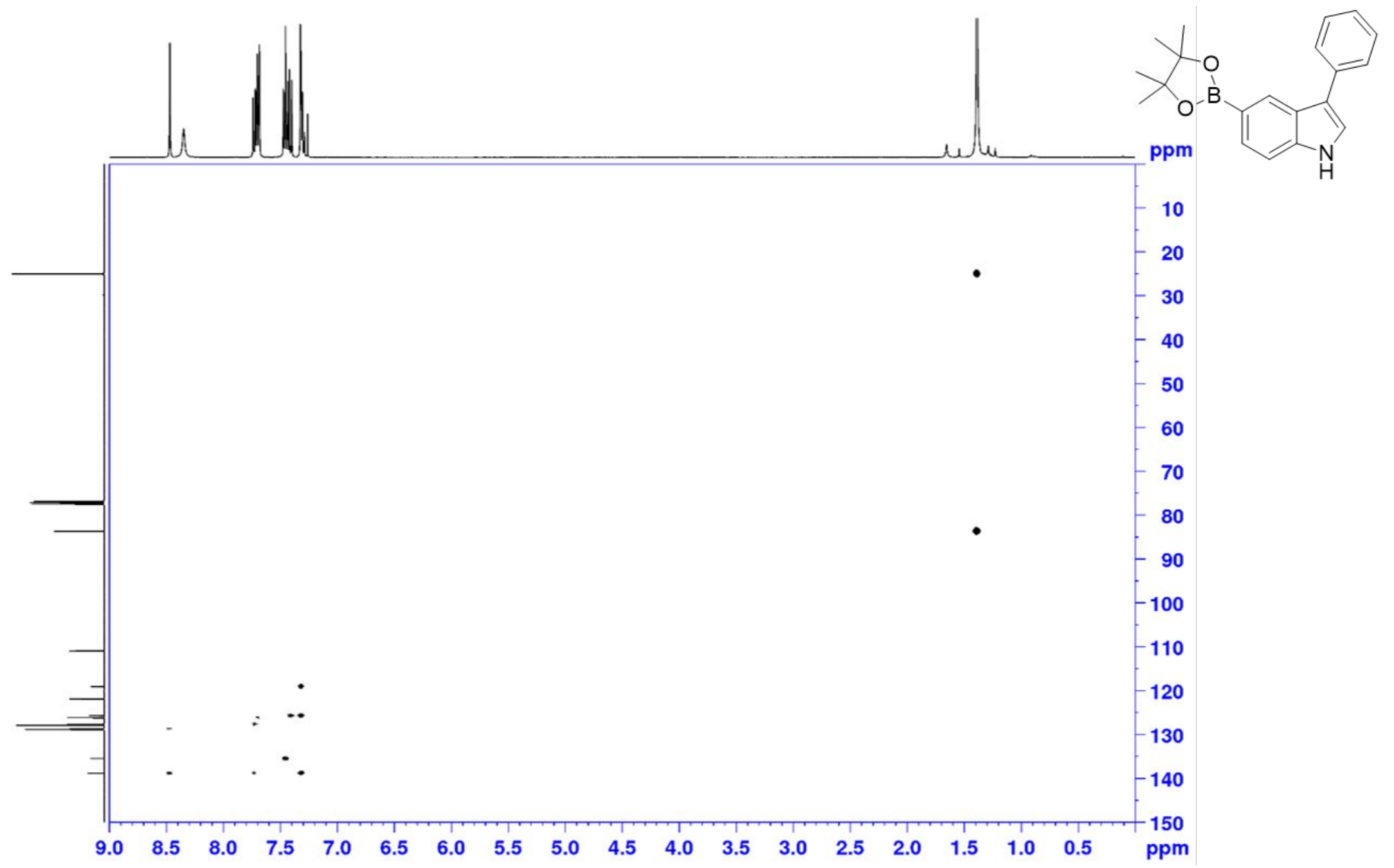

Figure S42: ${ }^{1} \mathrm{H}-{ }^{13} \mathrm{C}$ HMBC NMR spectrum of 3-phenyl-5-(4,4,5,5-tetramethyl-1,3,2-dioxaborolan-2-yl)-1 H-indole (400.13 $\left.\mathrm{MHz}, \mathrm{CDCl}_{3}\right)$. 

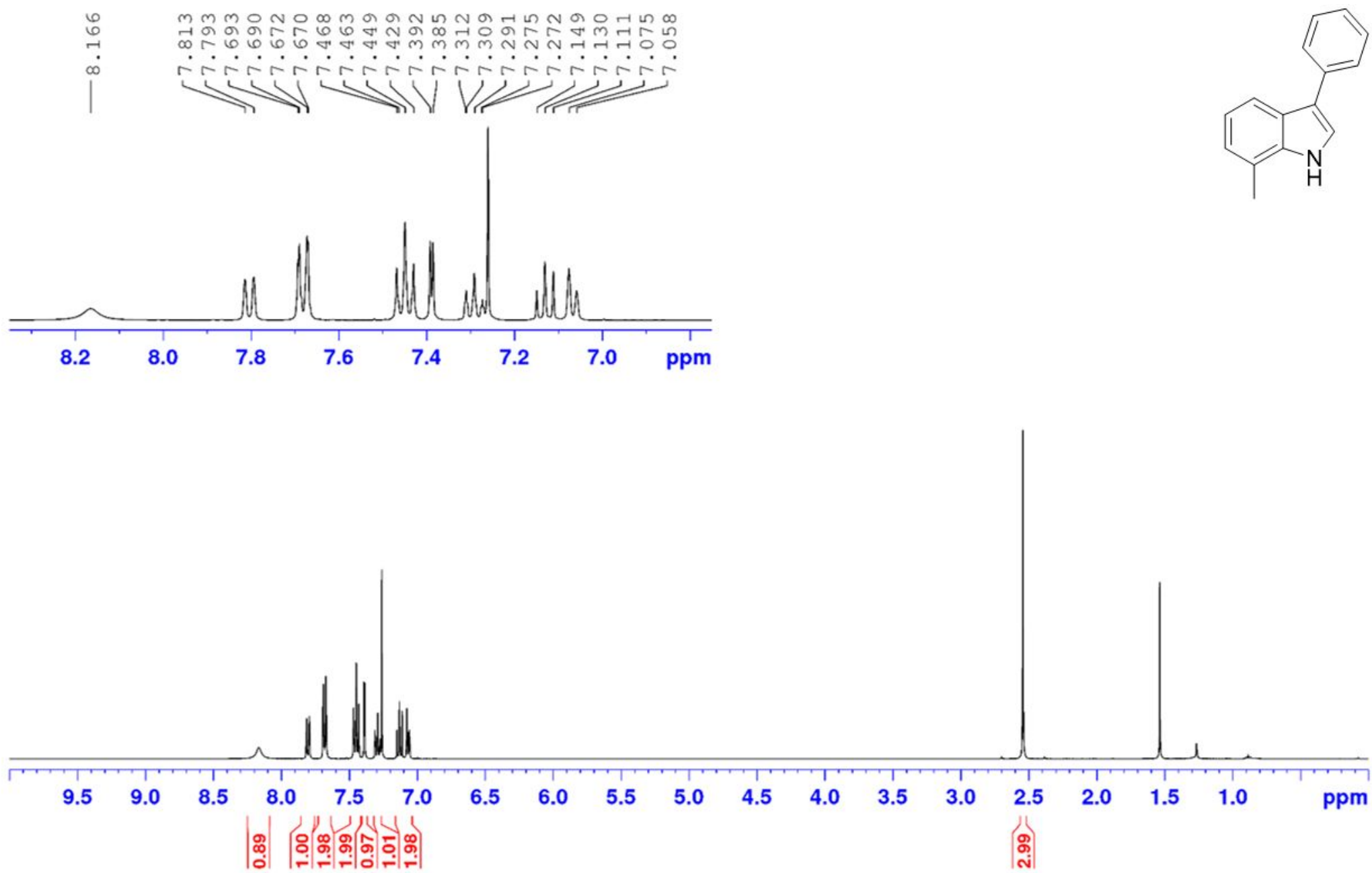

Figure S43: ${ }^{1} \mathrm{H}$ NMR spectrum of 7 -methyl-3-phenyl-1 1 -indole $\left(400.13 \mathrm{MHz}, \mathrm{CDCl}_{3}\right)$. 


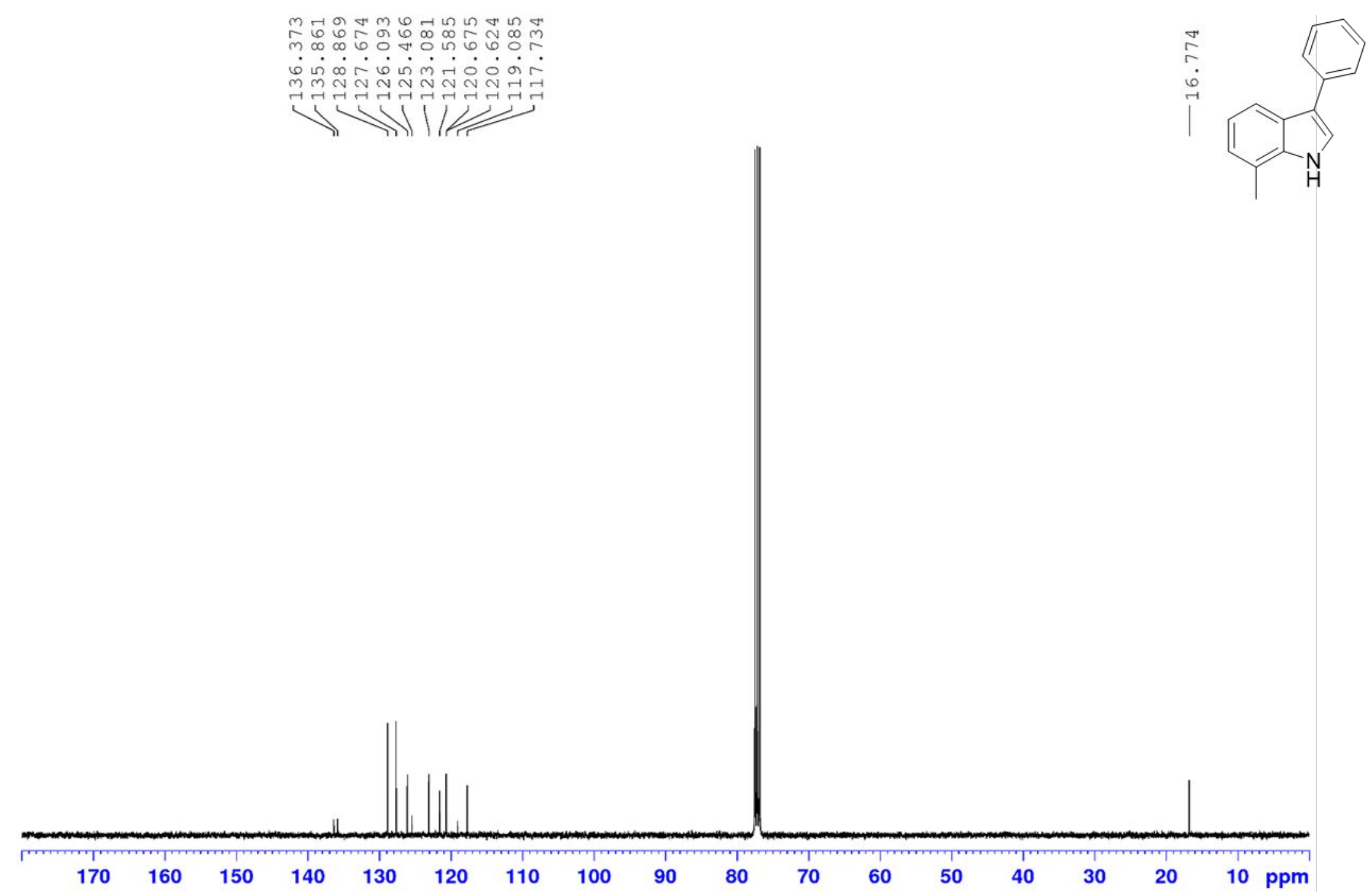

Figure S44: ${ }^{13} \mathrm{C}$ NMR spectrum of 7 -methyl-3-phenyl-1 $\mathrm{H}$-indole $\left(100.61 \mathrm{MHz}, \mathrm{CDCl}_{3}\right)$. 

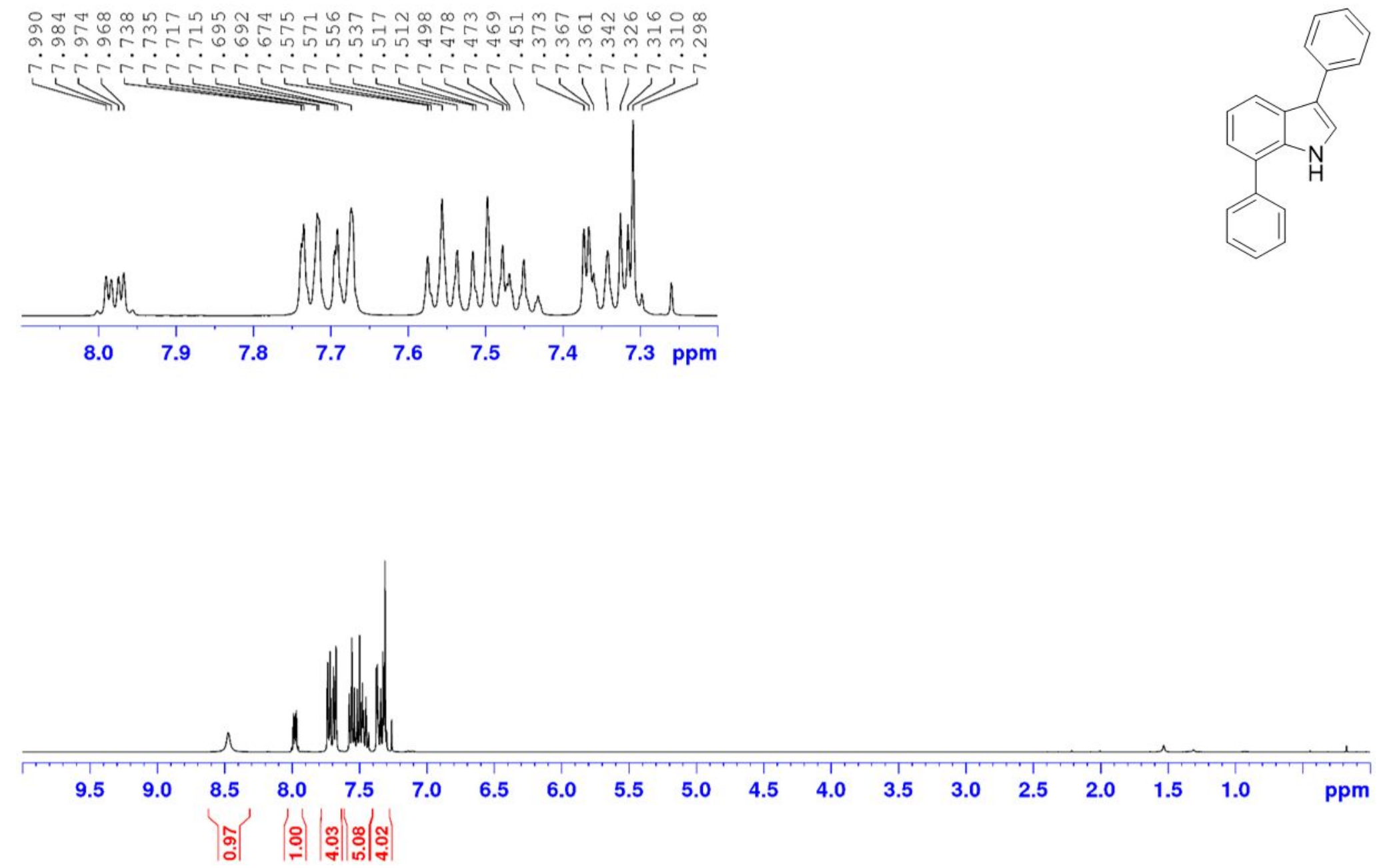

Figure S45: ${ }^{1} \mathrm{H}$ NMR spectrum of 3,7-diphenyl-1H-indole $\left(400.13 \mathrm{MHz}, \mathrm{CDCl}_{3}\right)$. 


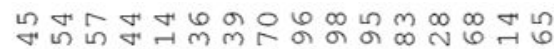

न 66 m ब

m

$\longrightarrow+1$
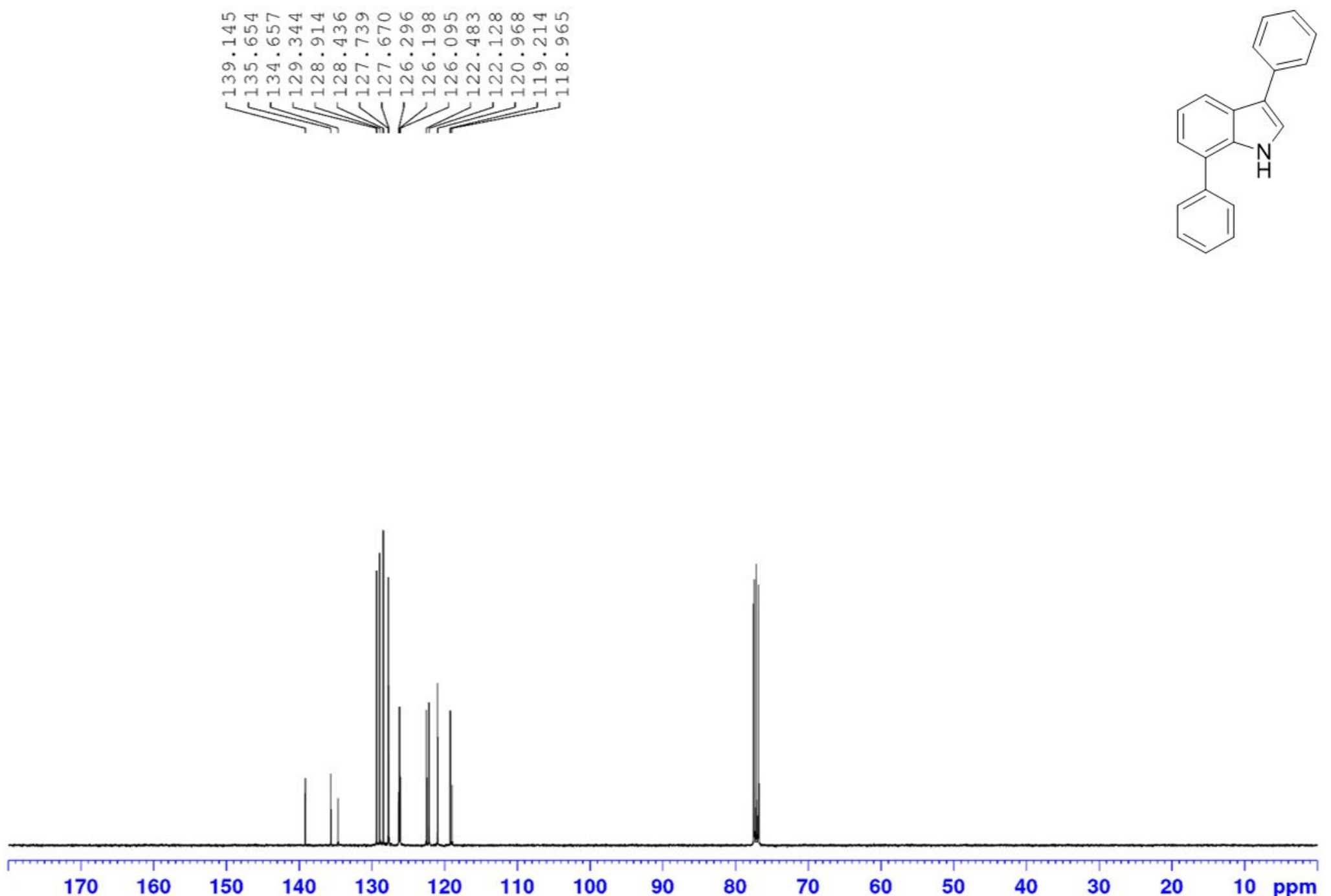

Figure S46: ${ }^{13} \mathrm{C}$ NMR spectrum of 3,7-diphenyl-1H-indole $\left(100.61 \mathrm{MHz}, \mathrm{CDCl}_{3}\right)$ 


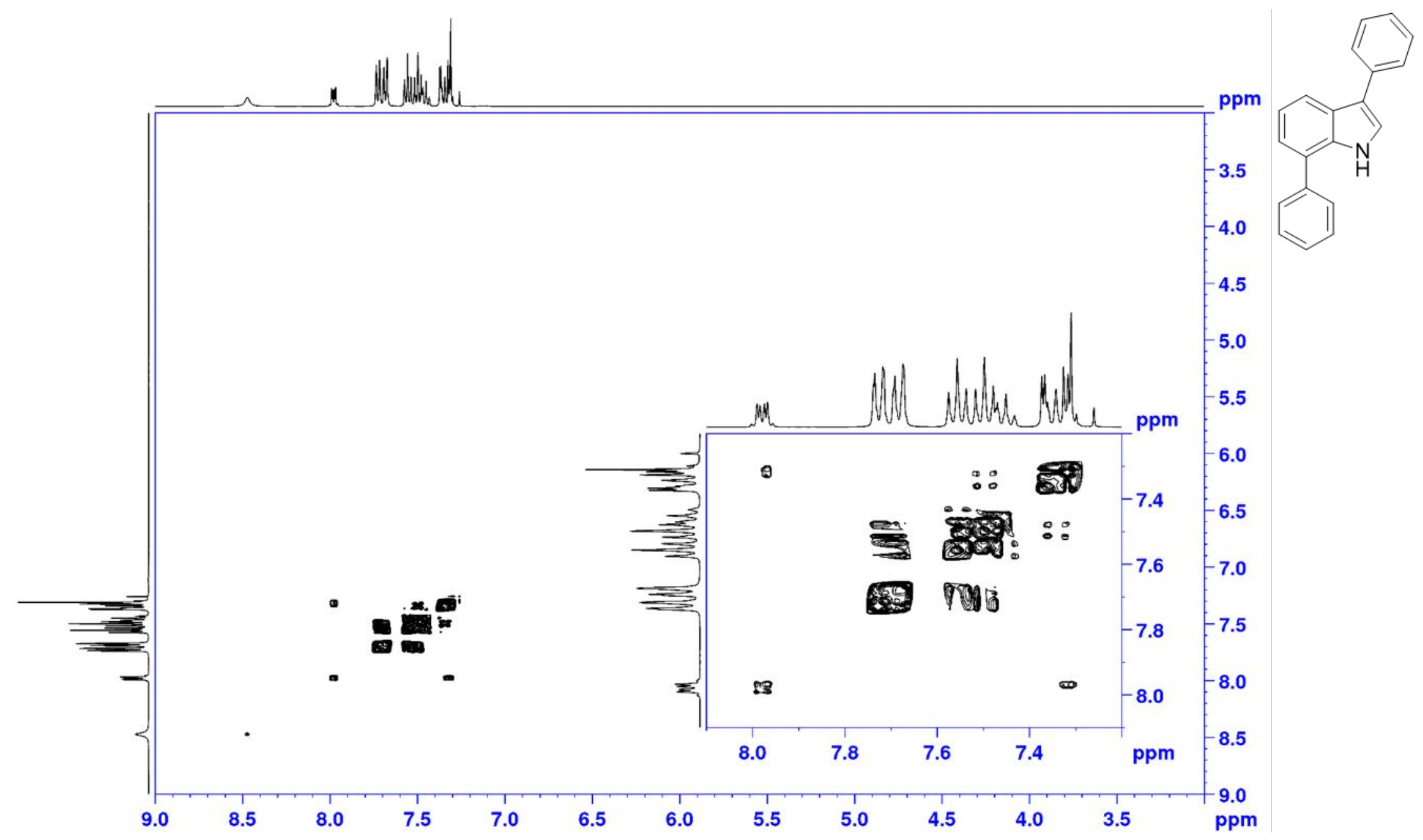

Figure S47: ${ }^{1} \mathrm{H}-{ }^{1} \mathrm{H}$ COSY NMR spectrum of 3,7-diphenyl-1 $\mathrm{H}$-indole $\left(400.13 \mathrm{MHz}, \mathrm{CDCl}_{3}\right.$ ). 


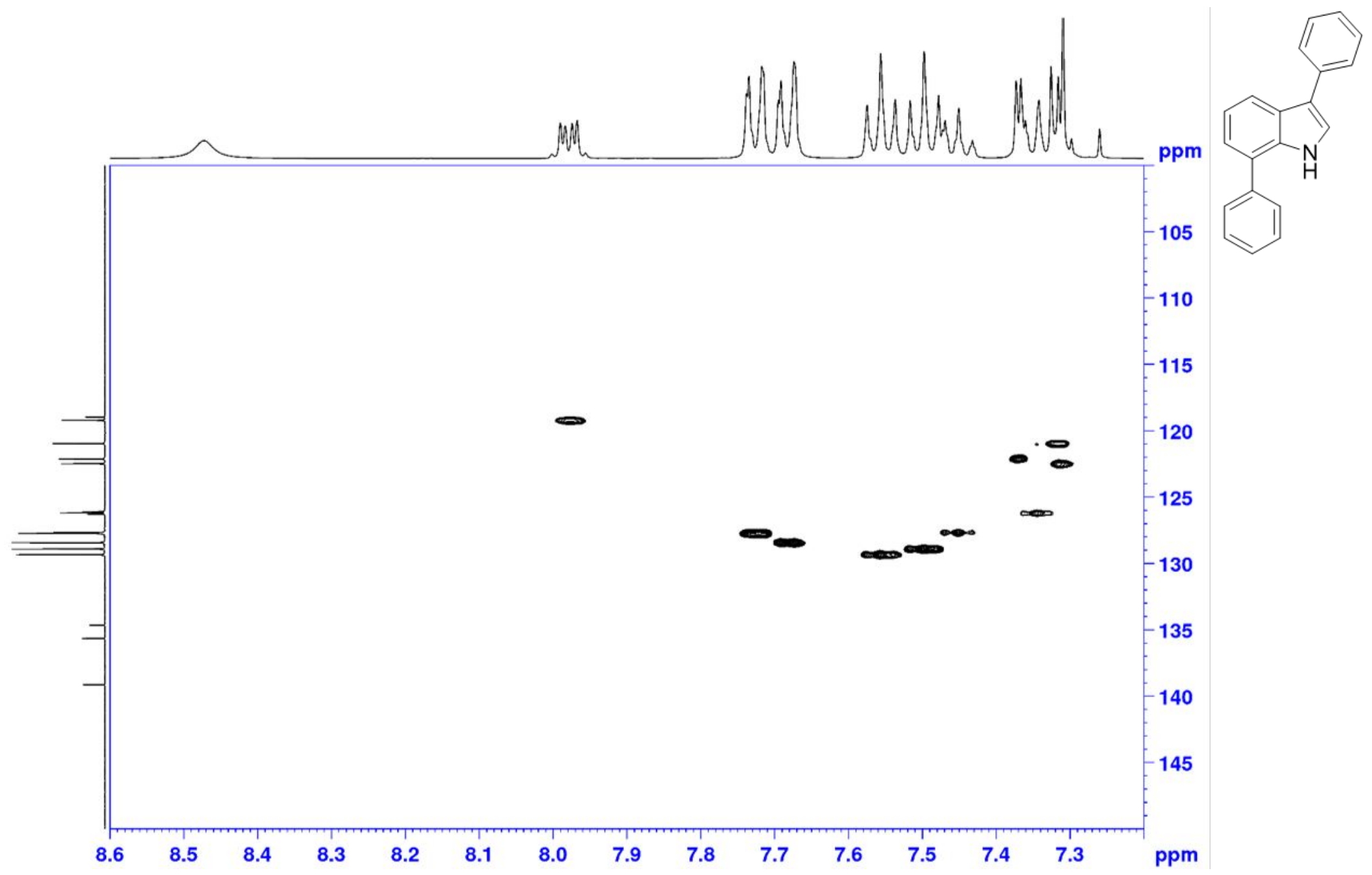

Figure S48: ${ }^{1} \mathrm{H}-{ }^{13} \mathrm{C}$ HSQC NMR spectrum of 3,7-diphenyl- $1 \mathrm{H}$-indole $\left(400.13 \mathrm{MHz}, \mathrm{CDCl}_{3}\right)$. 


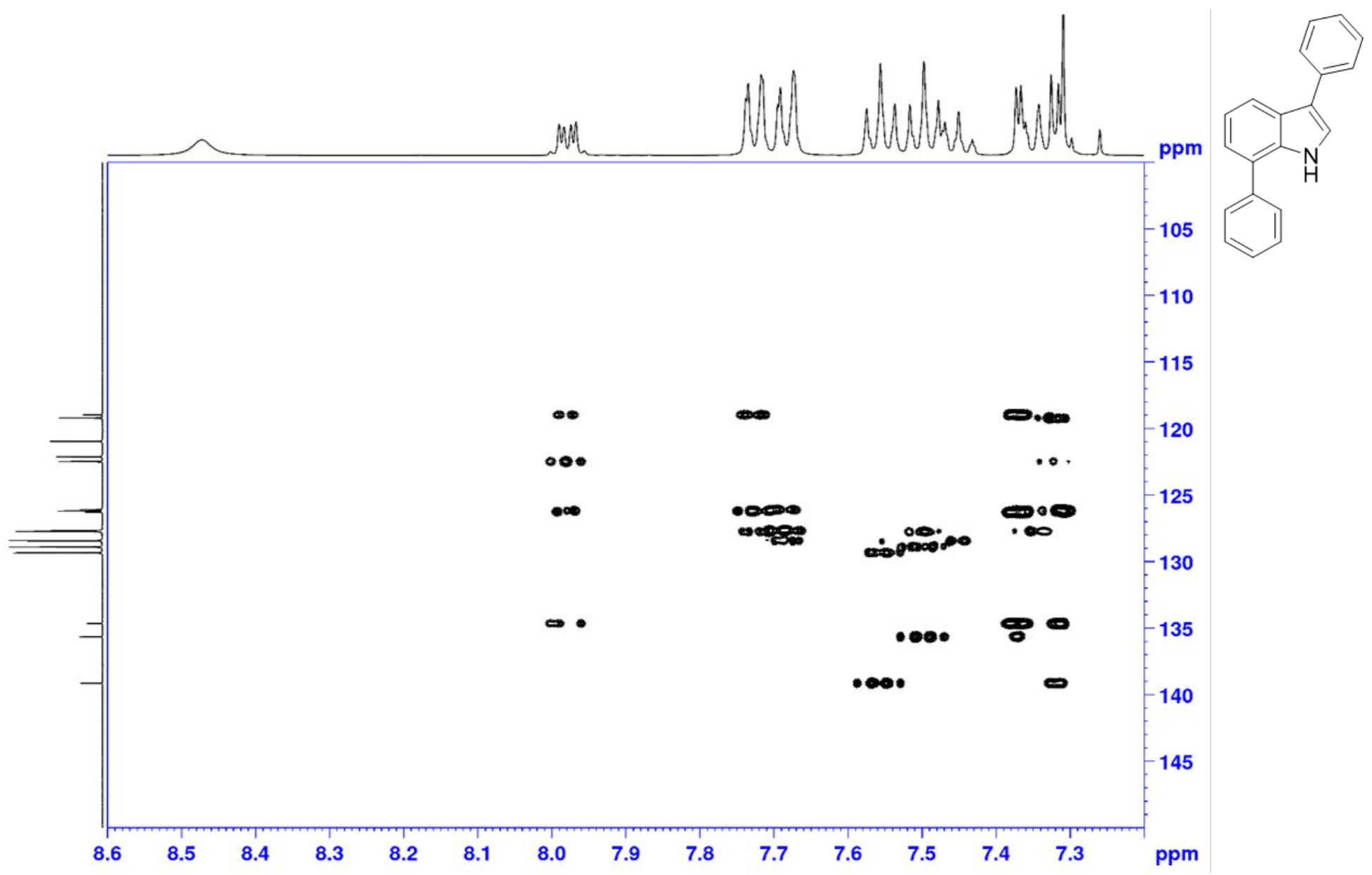

Figure S49: ${ }^{1} \mathrm{H}-{ }^{13} \mathrm{C}$ HMBC NMR spectrum of 3,7-diphenyl-1 $\mathrm{H}$-indole $\left(400.13 \mathrm{MHz}, \mathrm{CDCl}_{3}\right.$ ). 

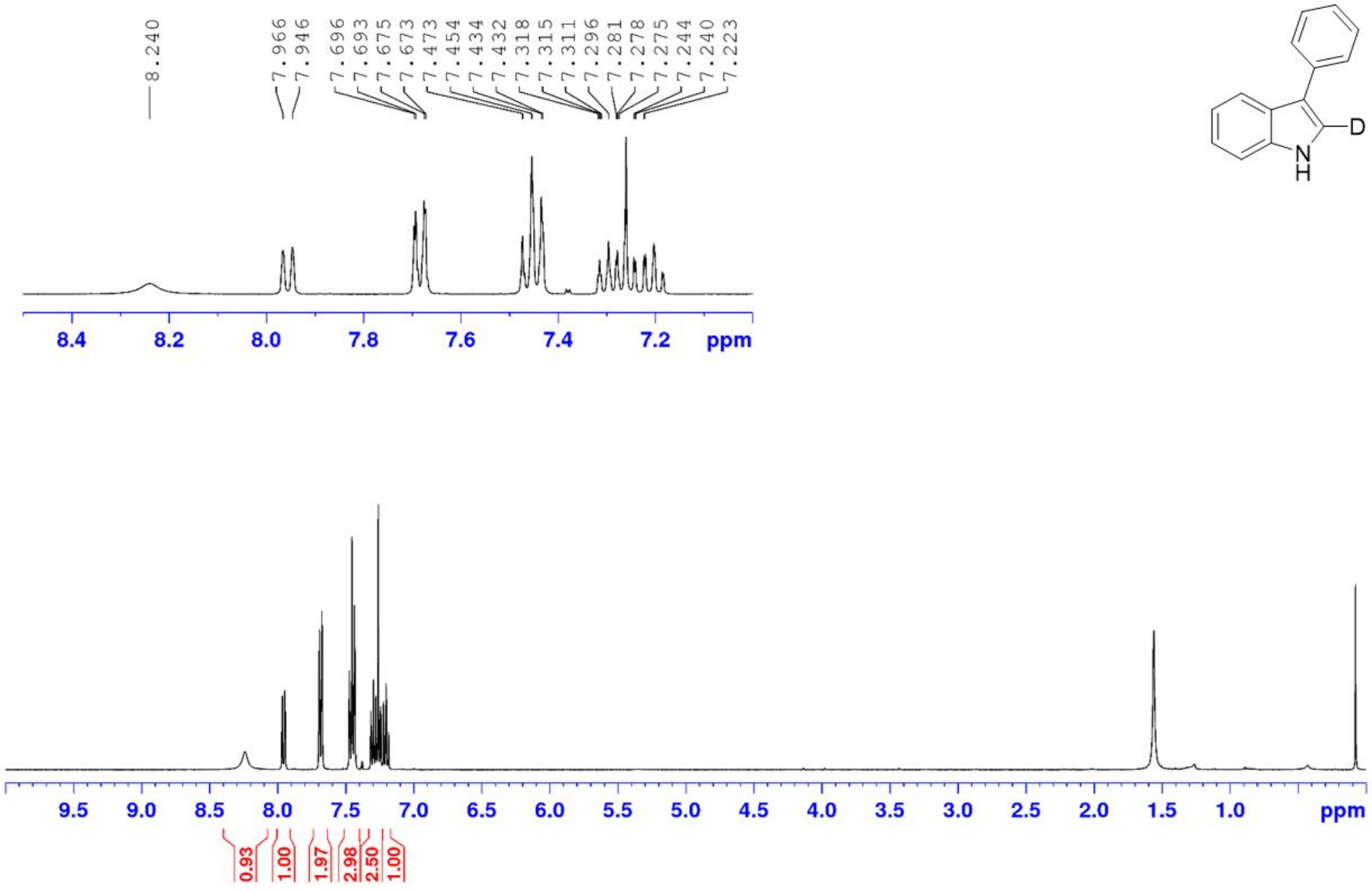

Figure S50: ${ }^{1} \mathrm{H}$ NMR spectrum of 3-phenyl-(2-2 $\left.\mathrm{H}\right)-1 \mathrm{H}$-indole $\left(400.13 \mathrm{MHz}, \mathrm{CDCl}_{3}\right)$. 


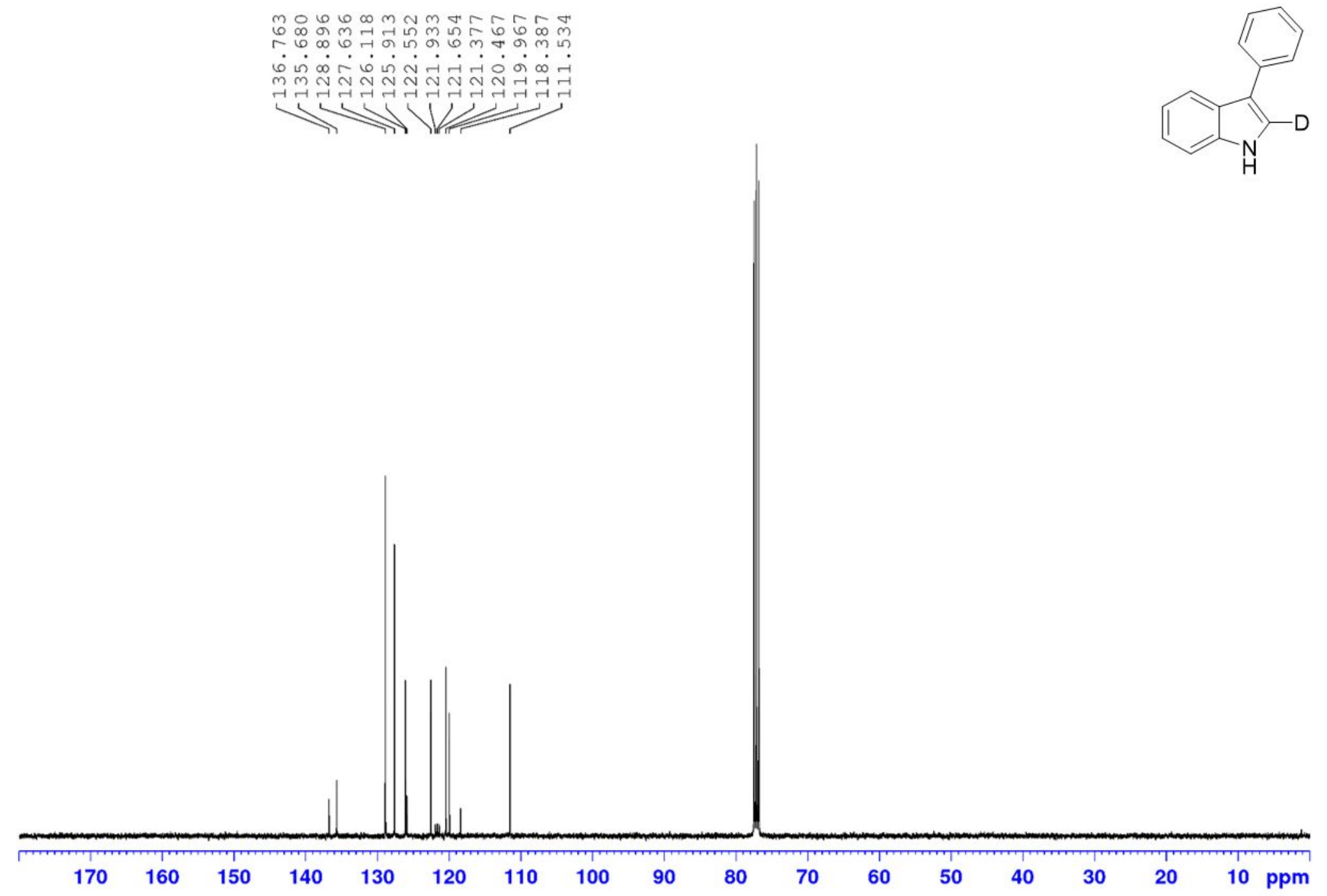

Figure S51: ${ }^{13} \mathrm{C}$ NMR spectrum of 3-phenyl-(2-2 $\left.\mathrm{H}\right)-1 \mathrm{H}$-indole $\left(400.13 \mathrm{MHz}, \mathrm{CDCl}_{3}\right)$. 


\section{Lithium based intermediate}

\subsection{Solubility of various Li-based species}

To a flame-dried Schlenk tube equipped with a magnetic stir bar was added indole $(0.5 \mathrm{mmol}$, $59 \mathrm{mg}$ ) and a lithium base in a glove box. The flask was sealed with a silicon septum and transferred out of the glove box. If $n$-BuLi was used a solution of that base in hexanes (c $=$ $2.5 \mathrm{~mol} / \mathrm{L})$ was injected outside of the glove box through the septum. Dry toluene $(4 \mathrm{~mL})$ was injected through the septum and the mixture was stirred for $10 \mathrm{~min}$ at room temperature (rt). In all cases a precipitate formed immediately. The tube was then heated to $120^{\circ} \mathrm{C}$ for $1 \mathrm{~h}$ and a possible change in solubility and color was noted down.
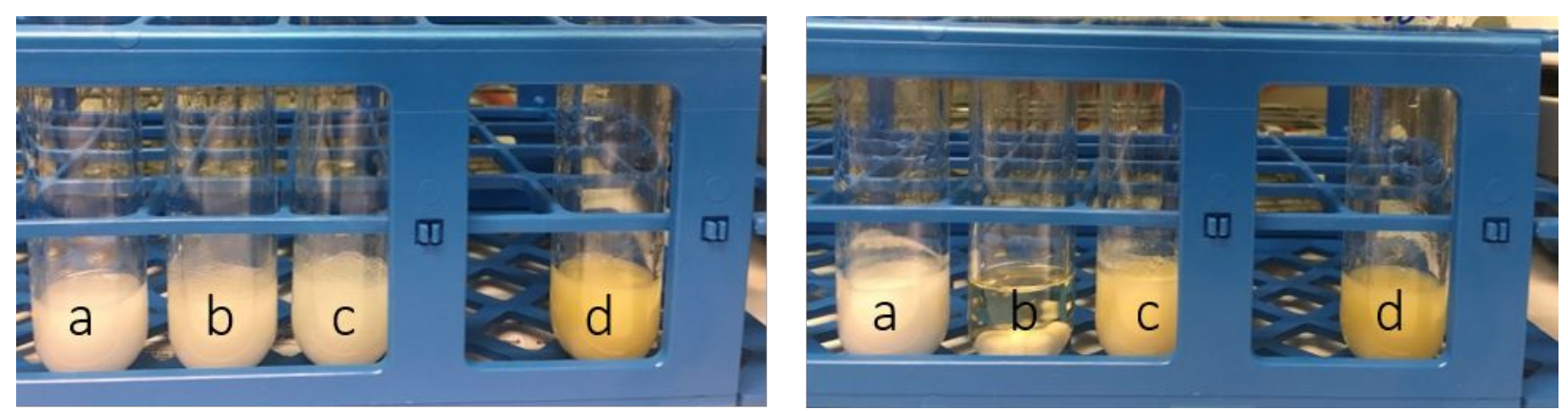

Figure S52: Different combinations of lithium bases and indole in toluene at room temperature (left) and after heating to $120^{\circ} \mathrm{C}$ for $1 \mathrm{~h}$ (right); a 1.0 equiv. of LiHMDS; $\mathbf{b} 2.2$ equiv. of LiHMDS; $\mathbf{c} 2.2$ equiv. of $n$-BuLi; $\mathbf{d} 2.2$ equiv. of LDA. 


\subsection{Synthesis of [indolide $\left.\cdot \mathrm{Li}_{2} \cdot \mathrm{HMDS}\right]$}<smiles>c1ccc2[nH]ccc2c1</smiles>
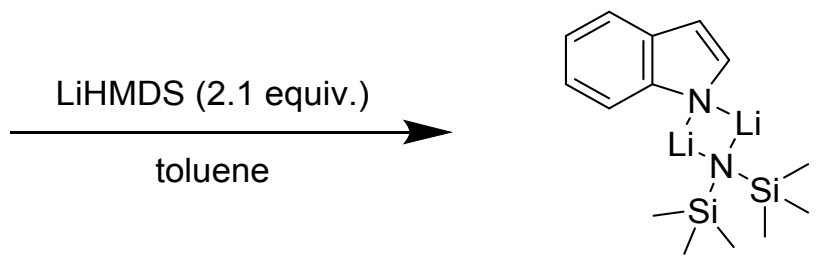

Indole (1 mmol, $118 \mathrm{mg}$ ), LiHMDS (350 mg, $2.1 \mathrm{mmol}, 2.1$ equiv.) and $8 \mathrm{~mL}$ of dry toluene were mixed in a vial inside the glovebox. A white precipitate formed immediately. The capped vial was warmed up to $120^{\circ} \mathrm{C}$ until complete solubilization of the precipitate and was then let to cool down to room temperature. The white precipitate was filtered and washed with dry toluene $(3 \times 10 \mathrm{~mL})$ and dry pentane $(2 \times 10 \mathrm{~mL})$ to remove excess of LiHMDS and the generated hexamethyldisilazane. The product was dried under vacuum. [Indolide $\cdot \mathrm{Li}_{2} \cdot \mathrm{HMDS}$ ] was obtained as a white solid in $94 \%$ isolated yield.

ICP-OES: calcd. for $\mathrm{C}_{14} \mathrm{H}_{24} \mathrm{Li}_{2} \mathrm{~N}_{2} \mathrm{Si}_{2}: 4.8$ wt.\% Li, found 4.9 wt. \% Li ( \pm 0.1 wt. \%).

Elemental Analysis: calcd. for $\mathrm{C}_{14} \mathrm{H}_{24} \mathrm{Li}_{2} \mathrm{~N}_{2} \mathrm{Si}_{2}$ (wt. \%): C 57.90, H 8.33, Li 4.78, N 9.65, Si 19.34; found: C 56.66, H 7.96, Li 4.62, N 9.38, Si 19.50.

NMR in toluene- $d_{8}$ at $105^{\circ} \mathrm{C}$

${ }^{1} \mathrm{H}$ NMR $\left(300.13 \mathrm{MHz}\right.$, toluene- $\left.d_{8}\right) \delta: 7.55(1 \mathrm{H}, \mathrm{d}, J=6.7 \mathrm{~Hz}), 7.07$ - $6.95(4 \mathrm{H}, \mathrm{m}), 6.47(1 \mathrm{H}, \mathrm{m}),-$ $0.03(18 \mathrm{H}, \mathrm{s})$.

${ }^{13} \mathrm{C}\left\{{ }^{1} \mathrm{H}\right\}$ NMR $\left(75.47 \mathrm{MHz}\right.$, toluene- $\left.d_{8}\right) \delta: 146.6,129.7,121.1,121.0,119.9,117.3,101.1,5.8$. (one missing aromatic peak presumably overlapping with residual solvent signals)

${ }^{7}$ Li NMR (116.64 MHz, toluene- $\left.d_{8}\right) \delta:-1.47$.

\section{Solid state NMR}

${ }^{13} \mathrm{C}\left\{{ }^{1} \mathrm{H}\right\}$ CPMAS NMR $(125.73 \mathrm{MHz}) \delta: 148.9,138.2,124.4,122.6,121.7,121.0,118.0,90.8,7.8$.

7Li MAS NMR (194.33 MHz) ס: -2.5. 


\subsection{In-situ formation and solubilization of [indolide $\cdot \mathrm{Li}_{2} \cdot \mathrm{HMDS}$ ]}

To an NMR tube was added indole ( $3.5 \mathrm{mg}, 0.03 \mathrm{mmol}$ ) and LiHMDS (10 mg, $0.06 \mathrm{mmol})$ in a glove box. The tube was sealed with a Young cap and transferred out of the glove box. Dry deuterated toluene- $d_{8}(0.4 \mathrm{~mL})$ was injected. A white precipitate formed immediately. ${ }^{1} \mathrm{H}$ and ${ }^{7} \mathrm{Li}$ NMR spectra were recorded at room temperature, at $60^{\circ} \mathrm{C}$ and at $105^{\circ} \mathrm{C}$.
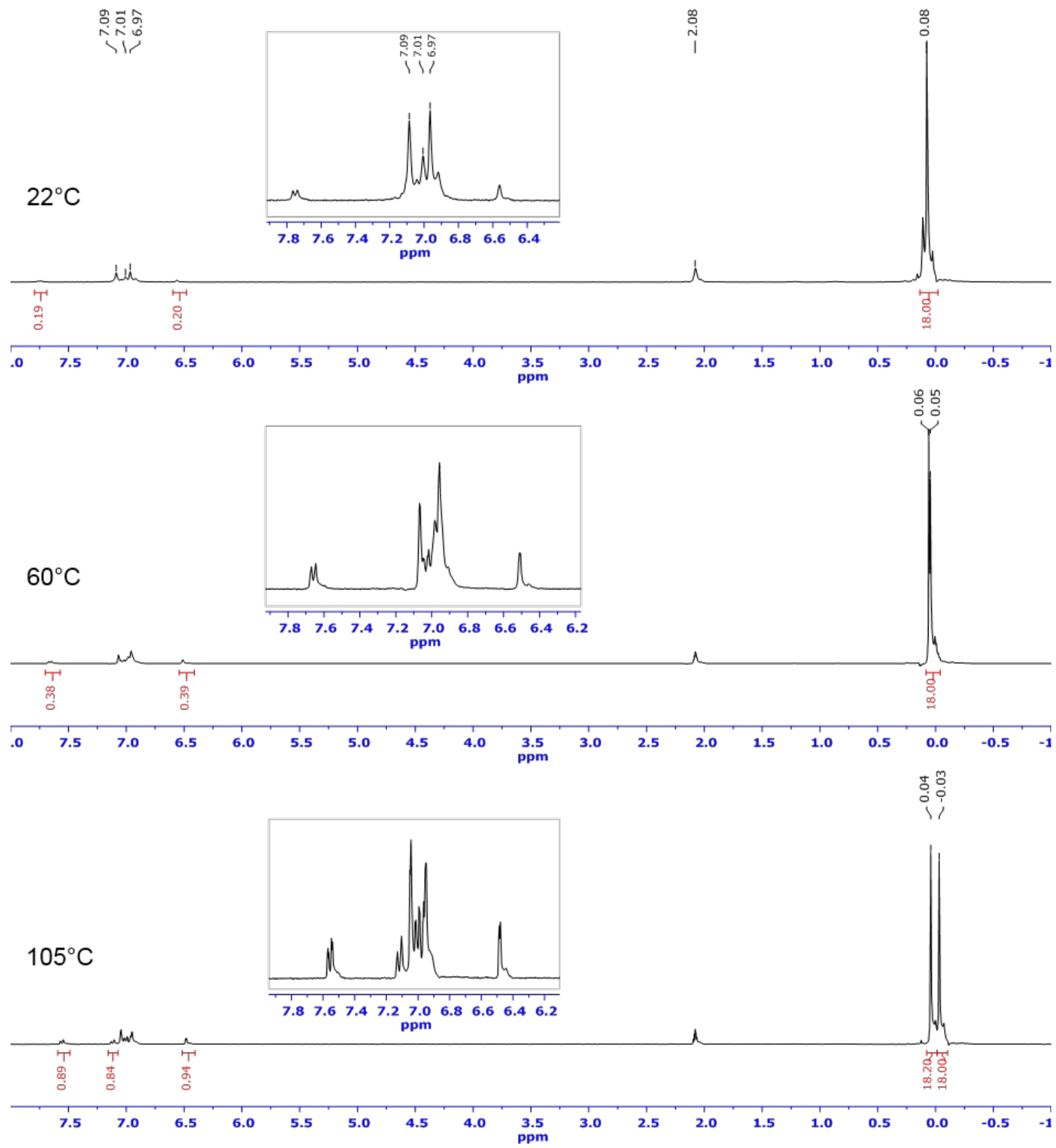

Figure S53: ${ }^{1} \mathrm{H}$ NMR spectra of in-situ formed [indolide $\cdot \mathrm{Li}_{2} \cdot \mathrm{HMDS}$ ] at different temperatures $(300.13 \mathrm{MHz}$, toluene$d_{8}$ ). Residual solvent signals appear at $\delta: 7.09,7.01,6.97,2.08$. 


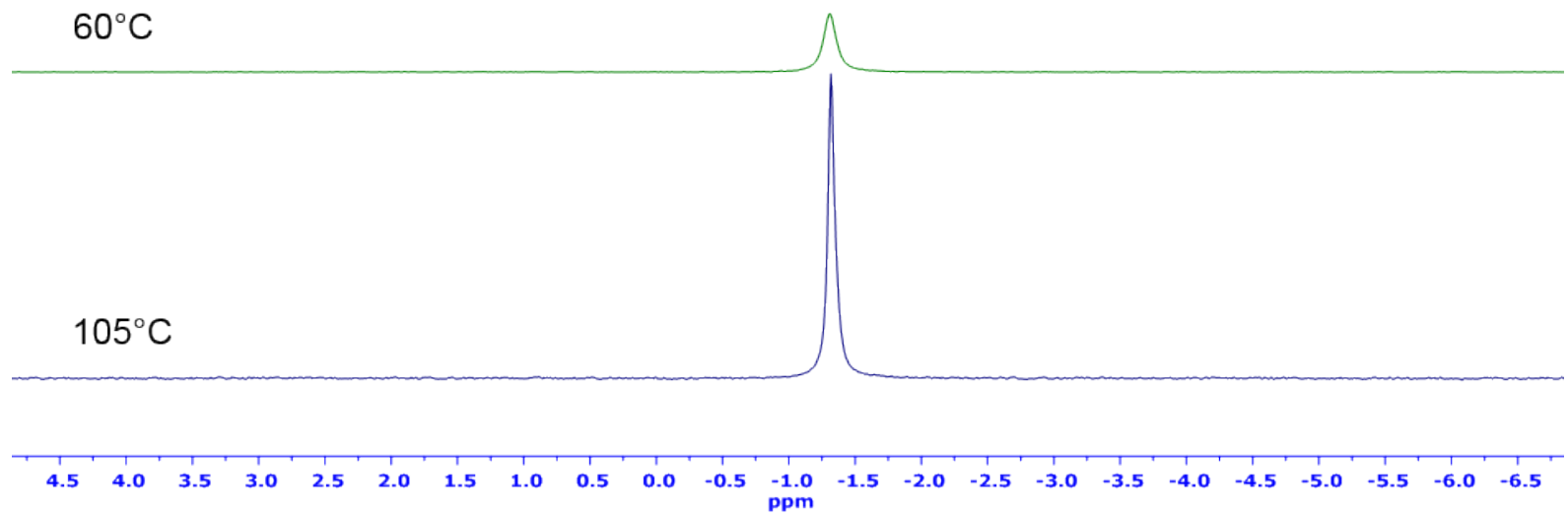

Figure S54: ${ }^{7} \mathrm{Li} \mathrm{NMR}$ spectra of in-situ formed [indolide $\cdot \mathrm{Li}_{2} \cdot \mathrm{HMDS}$ ] at different temperatures $(116.64 \mathrm{MHz}$, toluene$d_{8}$ ). In all cases the same number of scans was used. 


\subsection{NMR spectra of [indolide $\cdot \mathrm{Li}_{2} \cdot \mathrm{HMDS}$ ]}

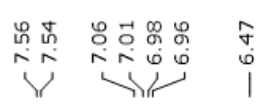

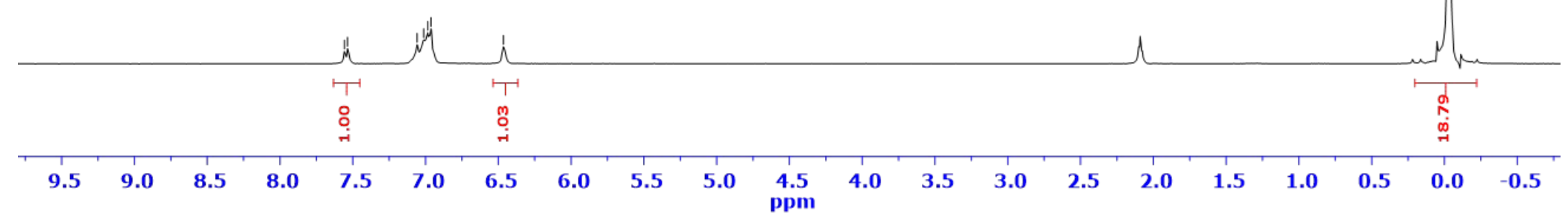

Figure S55: ${ }^{1} \mathrm{H}$ NMR spectrum of [indolide $\left.\cdot \mathrm{Li}_{2} \cdot \mathrm{HMDS}\right]\left(300.13 \mathrm{MHz}\right.$, toluene- $\left.d_{8}, 105^{\circ} \mathrm{C}\right)$. 


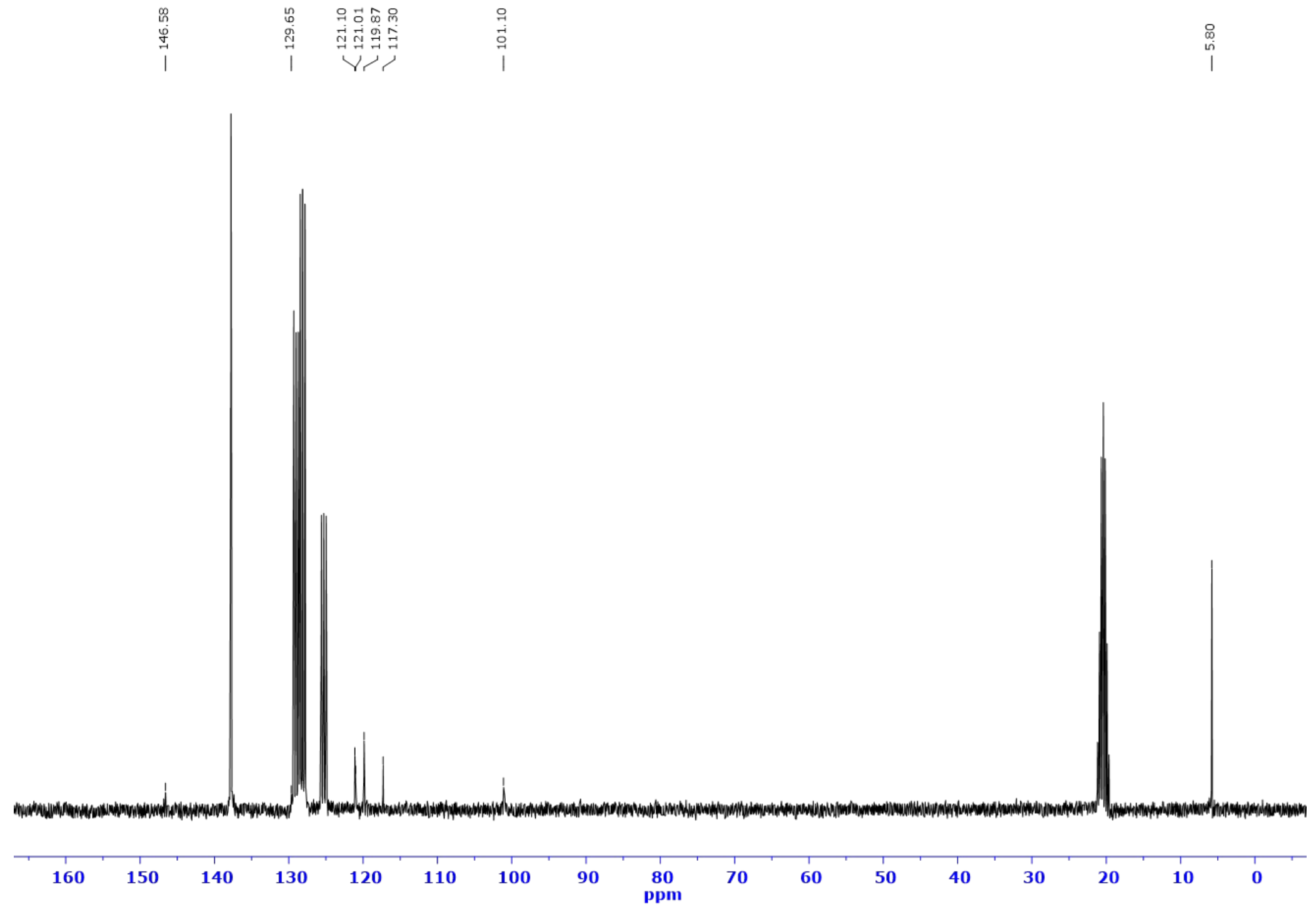

Figure S56: ${ }^{13} \mathrm{C}$ NMR spectrum of [indolide $\left.\cdot \mathrm{Li}_{2} \cdot \mathrm{HMDS}\right]\left(75.47 \mathrm{MHz}\right.$, toluene- $\left.d_{8}, 105^{\circ} \mathrm{C}\right)$ 


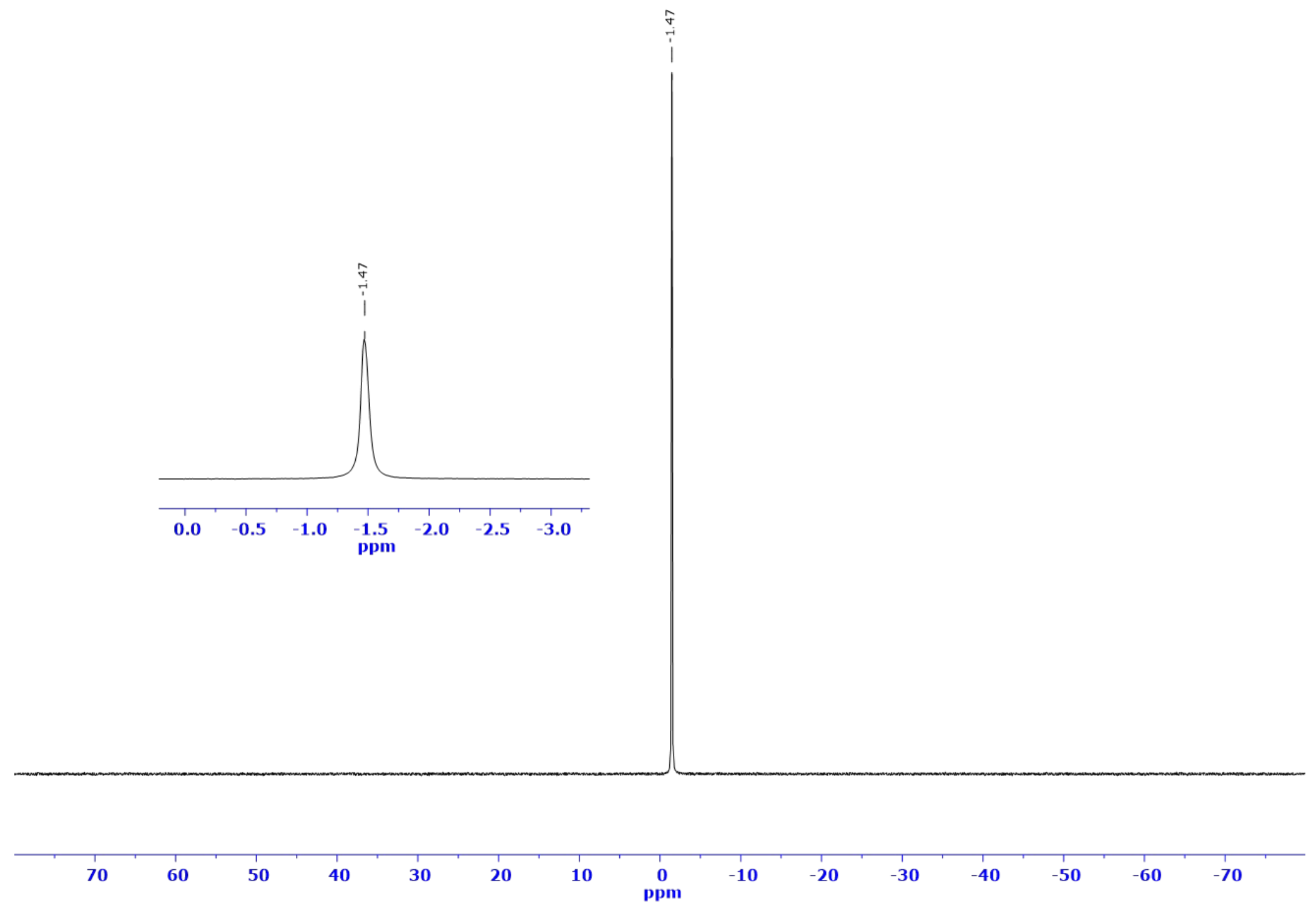

Figure S57: ${ }^{7} \mathrm{Li}$ NMR spectrum of [indolide $\left.\cdot \mathrm{Li}_{2} \cdot \mathrm{HMDS}\right]\left(116.64 \mathrm{MHz}\right.$, toluene- $\left.d_{8}, 105^{\circ} \mathrm{C}\right)$ 


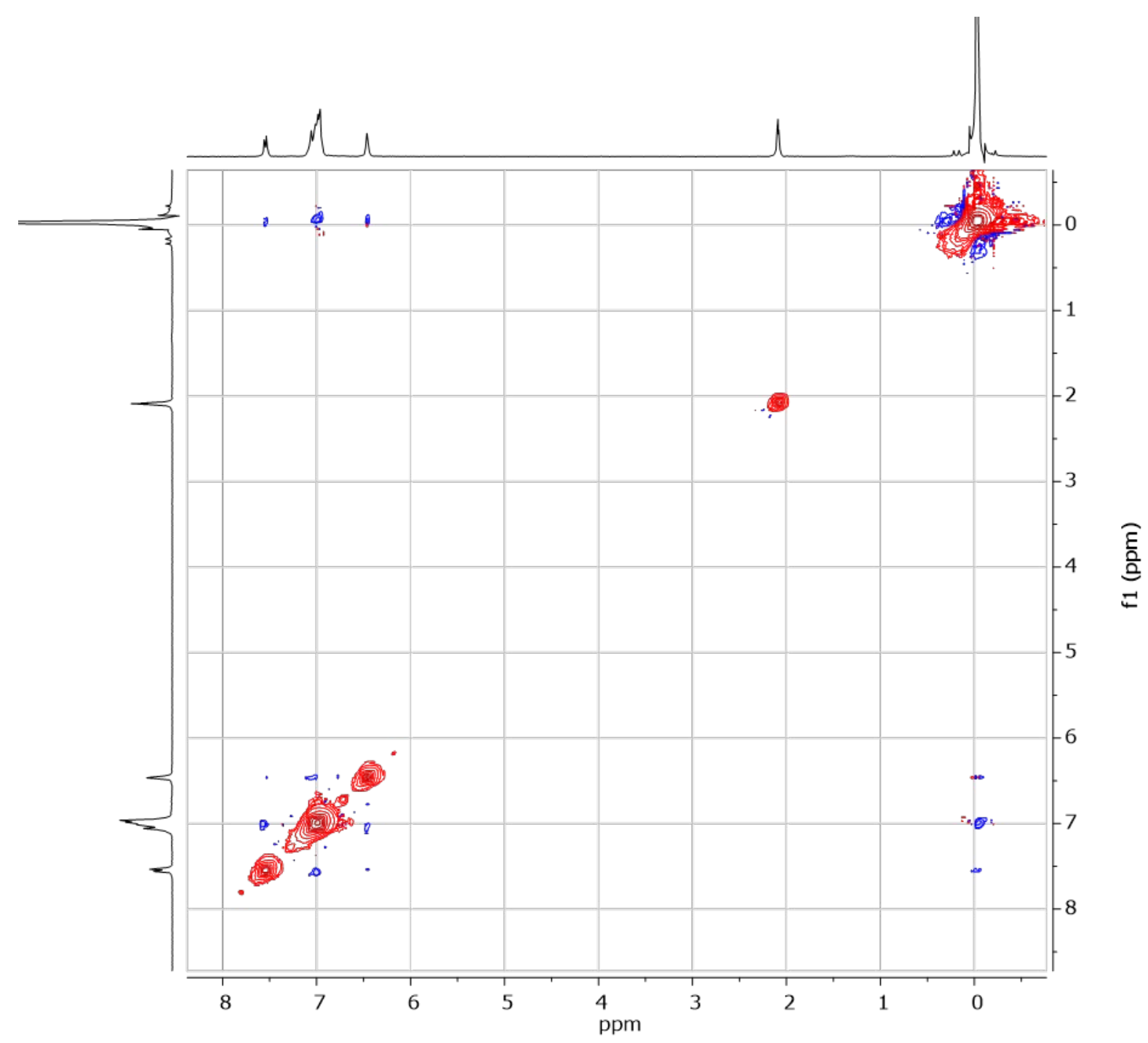

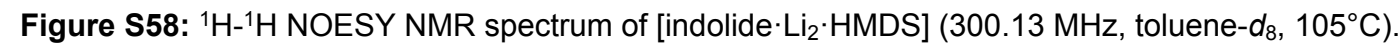




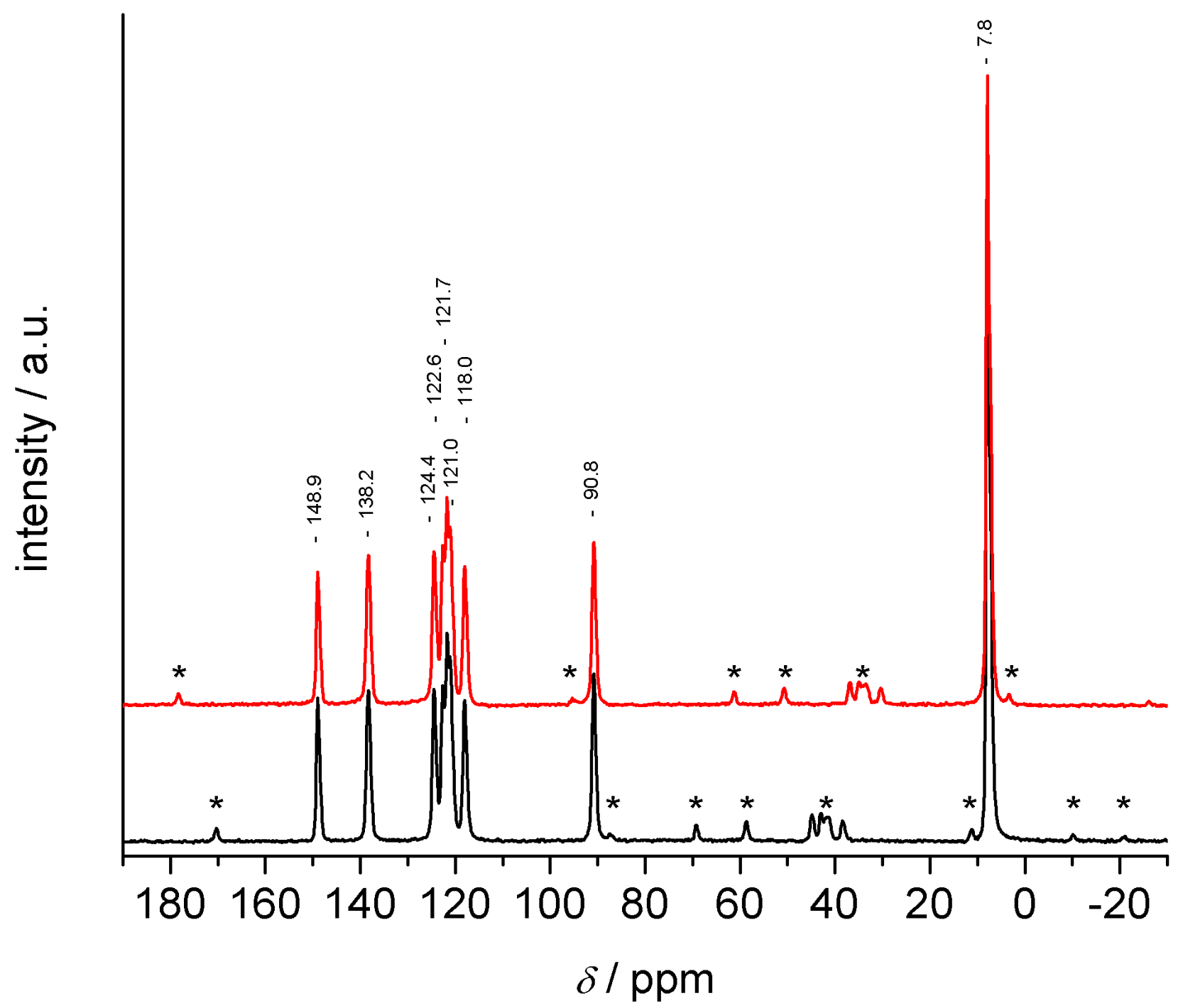

Figure S59: Superimposition of the ${ }^{13} \mathrm{C}$ CP MAS NMR spectra of [indolide $\cdot \mathrm{Li}_{2} \cdot \mathrm{HMDS}$ ] at $10 \mathrm{kHz}$ (black) and $11 \mathrm{kHz}$ (red) rotation speed (each $2048 \mathrm{scans}$ ). Signals marked with an asterisk $\left({ }^{*}\right)$ correspond to spinning side bands. 


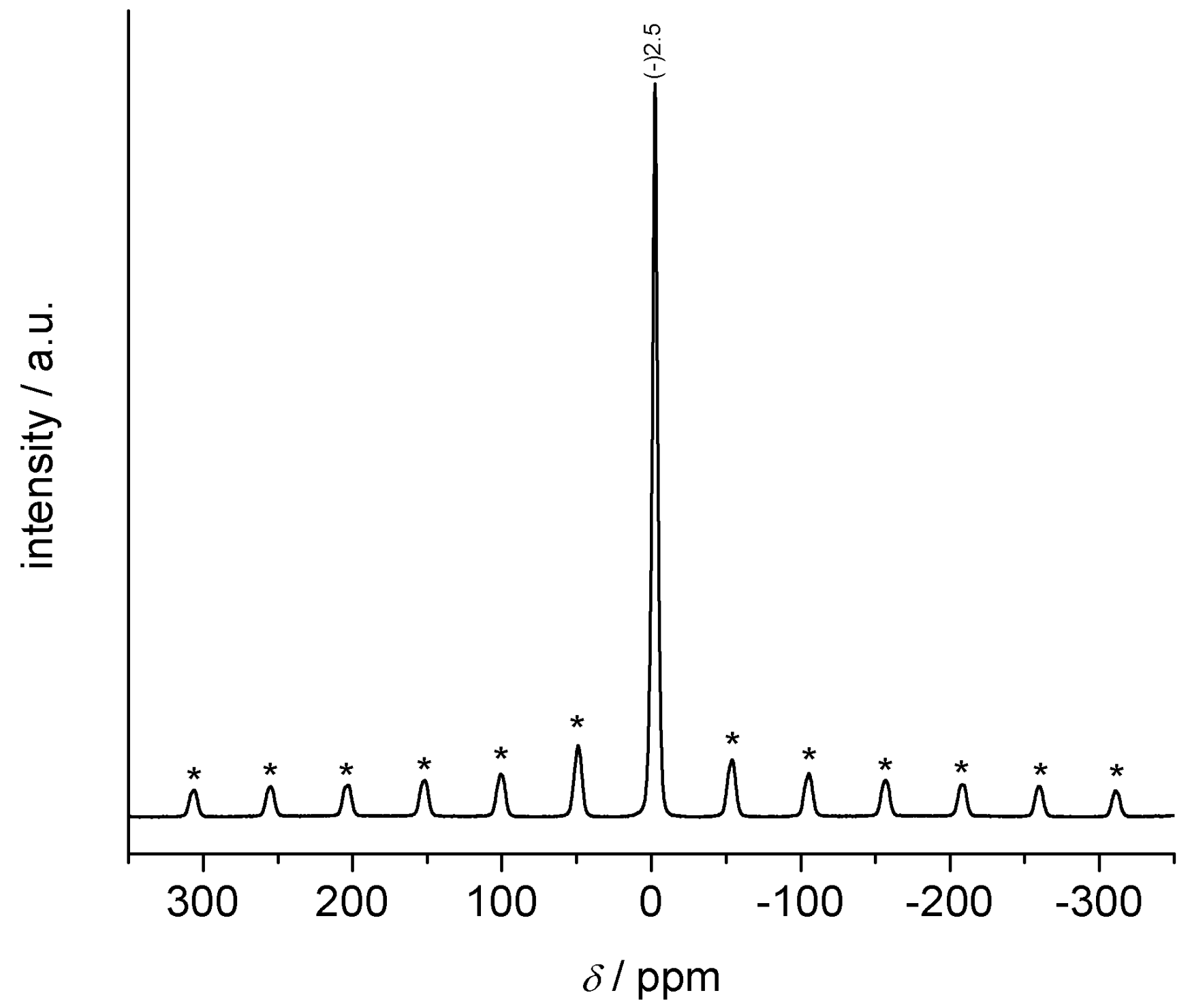

Figure S60: ${ }^{7} \mathrm{Li}$ MAS NMR spectrum of [indolide $\cdot \mathrm{Li}_{2} \cdot \mathrm{HMDS}$ (MAS $10 \mathrm{kHz}, 16384$ scans). Signals marked with an asterisk (*) correspond to spinning side bands. 


\subsection{NMR spectra of LiHMDS}

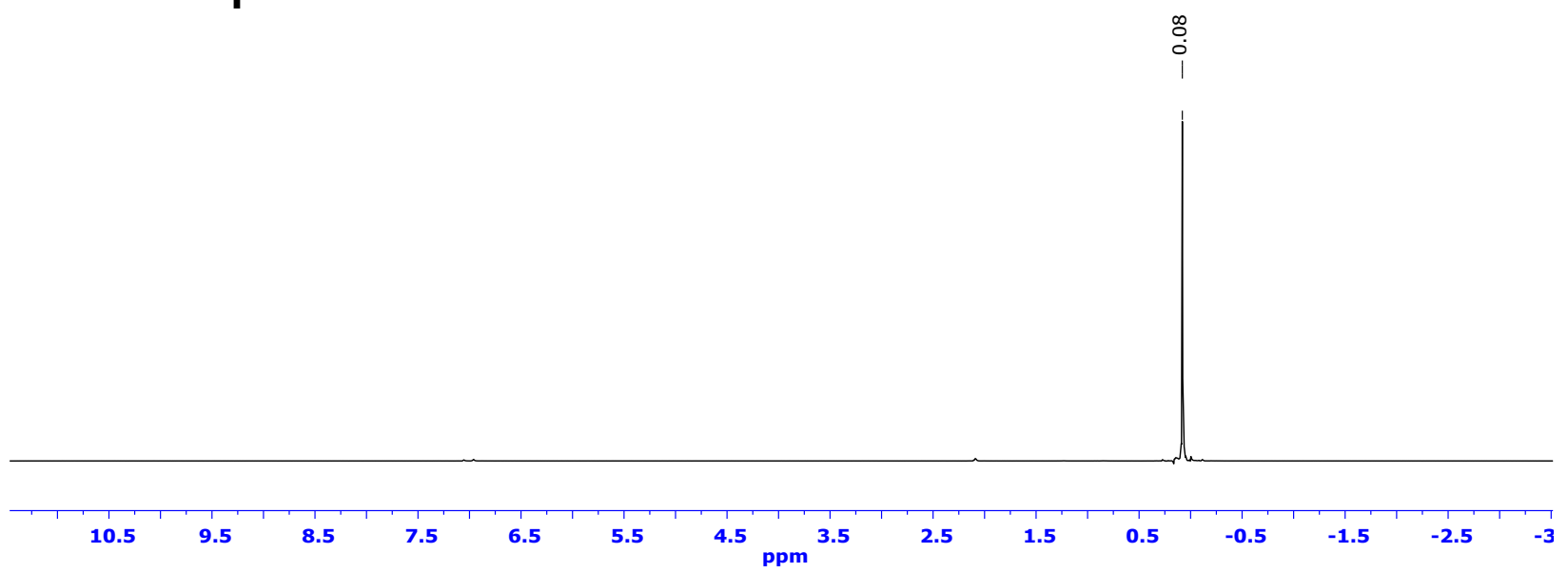

Figure S61: ${ }^{1} \mathrm{H}$ NMR spectrum of LiHMDS $\left(300.13 \mathrm{MHz}\right.$, toluene- $\left.d_{8}, 105^{\circ} \mathrm{C}\right)$.

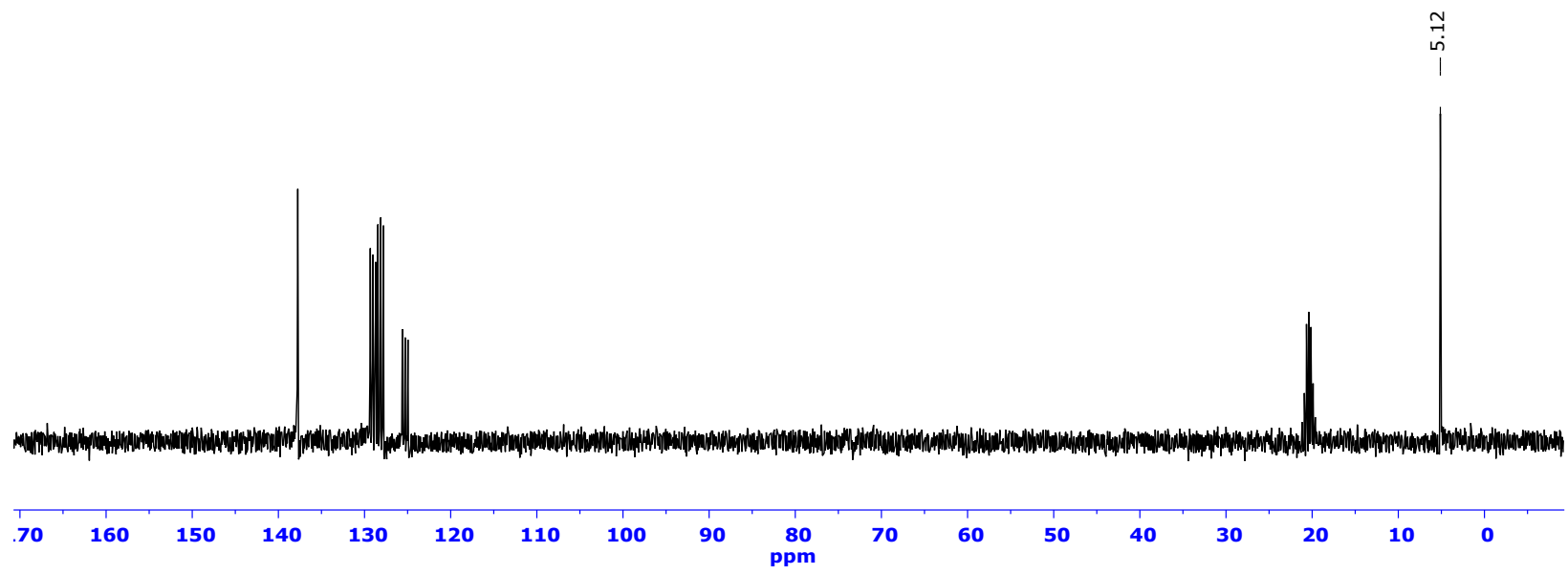

Figure S62: ${ }^{13} \mathrm{C}$ NMR spectrum of LiHMDS $\left(75.47 \mathrm{MHz}\right.$, toluene- $\left.\mathrm{d}_{8}, 105^{\circ} \mathrm{C}\right)$.

Figure S63: ${ }^{7} \mathrm{Li}$ NMR spectrum of LiHMDS $\left(116.64 \mathrm{MHz}\right.$, toluene- $\left.d_{8}, 105^{\circ} \mathrm{C}\right)$. 


\subsection{Catalytic test with [indolide $\cdot \mathrm{Li}_{2} \cdot \mathrm{HMDS}$ ]}<smiles>C[Si](C)(C)N([AlH])[Si](C)(C)n1ccc2ccccc21</smiles>

$0.5 \mathrm{mmol}$<smiles>Brc1ccccc1</smiles>

1.2 equiv.

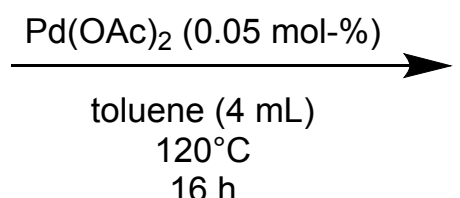

$16 \mathrm{~h}$

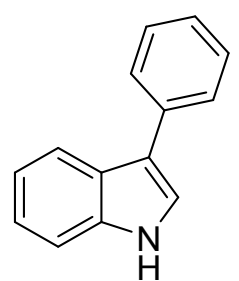

To a flame-dried Schlenk tube equipped with a magnetic stir bar was added in a glove box the [indolide $\cdot \mathrm{Li}_{2} \cdot \mathrm{HMDS}$ ] precipitate $(0.5 \mathrm{mmol}, 145 \mathrm{mg})$ obtained in section 5.2 . The flask was sealed with a silicon septum and transferred out of the glove box. Dry toluene $(4 \mathrm{~mL})$ was injected through the septum and the mixture was stirred for two minutes. Then a solution of $\operatorname{Pd}(O A c)_{2}$ in toluene (500 ppm, $50 \mu \mathrm{L}$ of stock solution 1) was injected through the septum along with bromobenzene ( $0.6 \mathrm{mmol}, 64 \mu \mathrm{L}, 1.2$ equiv.). The reaction was stirred at $120^{\circ} \mathrm{C}$. After $16 \mathrm{~h}$ the reaction mixture was quenched with $1 \mathrm{~mL}$ of deionized water. Then dodecane $(40 \mu \mathrm{L}, 0.174 \mathrm{mmol})$ as an internal standard for GC-FID analysis was added. The aqueous phase was extracted twice with $4 \mathrm{~mL}$ of ethyl acetate. The combined organic phases were washed with $2 \mathrm{~mL}$ of brine and dried over $\mathrm{MgSO}_{4}$. After removal of the solvent by rotary evaporation, the crude product was purified by liquid size exclusion chromatography to afford 3-phenyl- $1 H$-indole as a white solid in $85 \%$ isolated yield. The ${ }^{1} \mathrm{H}$ NMR spectrum is identical to the 3-phenyl-1H-indole obtained from General Procedure $\mathrm{C}$ (section 2.3). 


\section{Kinetic isotopic effect}

\subsection{Parallel reactions}

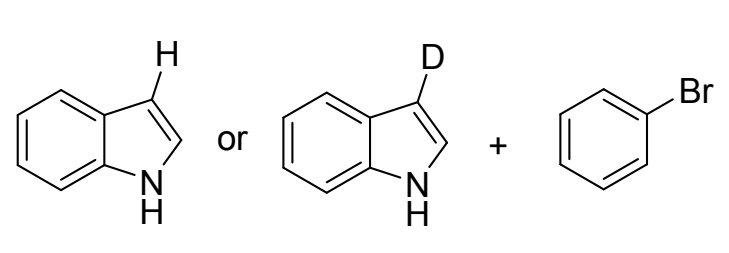

$0.5 \mathrm{mmol}$

1.2 equiv.
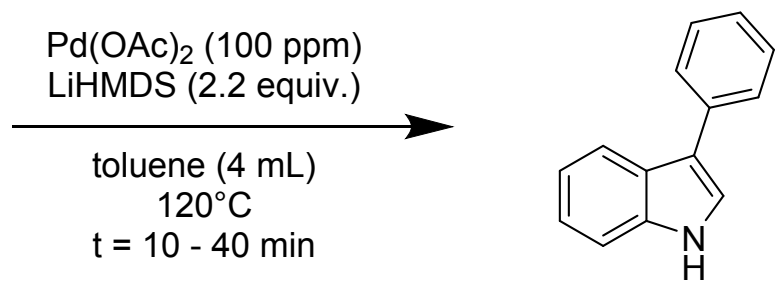

To a flame-dried Schlenk tube equipped with a magnetic stir bar was added indole or ( $3-2 \mathrm{H})$-indole $(0.5 \mathrm{mmol})$ in a glove box. The flask was sealed with a silicon septum and transferred out of the glove box. Dry toluene $(2.9 \mathrm{~mL})$ and a solution of LiHMDS in toluene (1 M, $1.1 \mathrm{~mL}, 2.2$ equiv.) were injected through the septum and the mixture was stirred for two minutes. Then a solution of $\mathrm{Pd}(\mathrm{OAc})_{2}$ in toluene (100 ppm, $10 \mu \mathrm{L}$ of stock solution 1) was injected through the septum along with bromobenzene $(0.6 \mathrm{mmol}, 64 \mu \mathrm{L}, 1.2$ equiv.) and dodecane $(40 \mu \mathrm{L}, 0.174 \mathrm{mmol})$. The reaction was stirred at $120^{\circ} \mathrm{C}$. After a given time the reaction mixture was quenched with $2 \mathrm{~mL}$ of methanol. The conversion was determined using GC-FID analysis against dodecane as internal standard.

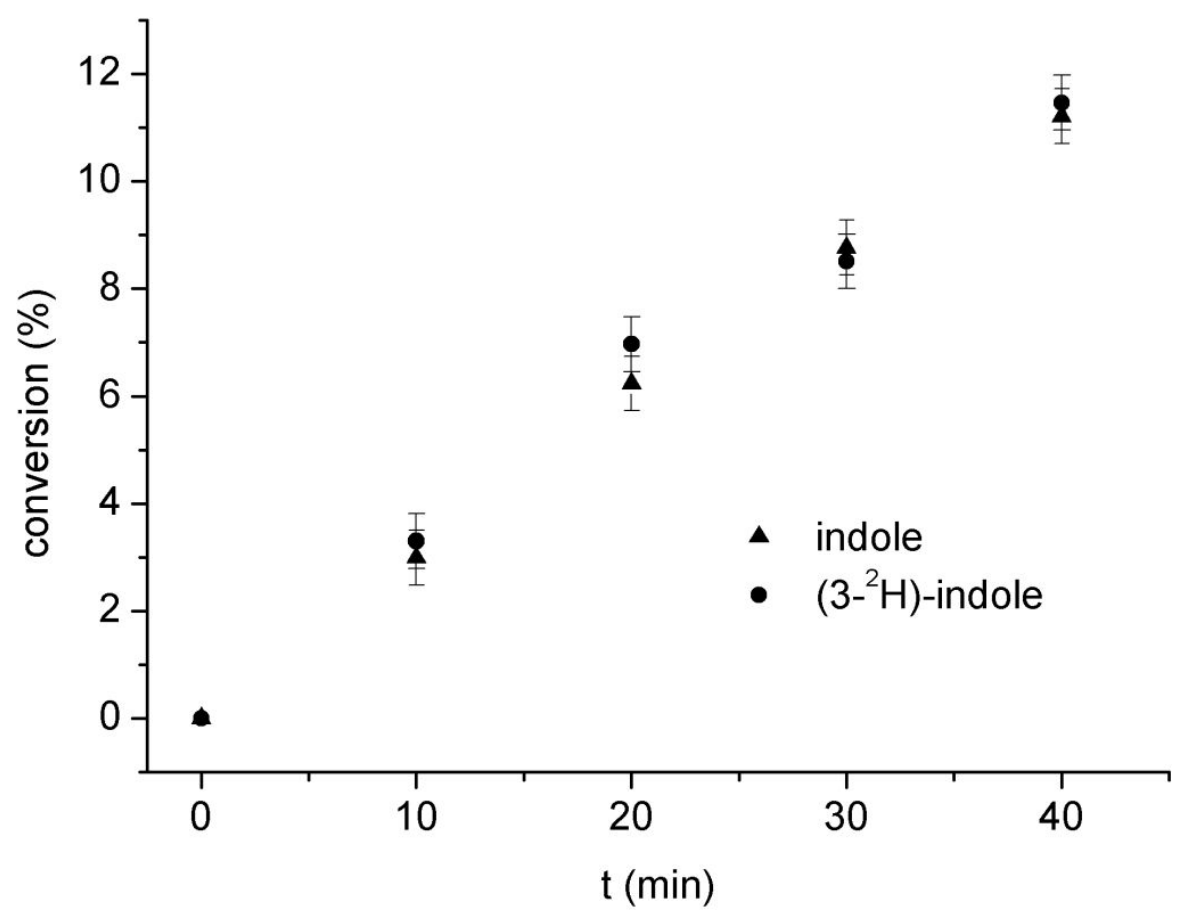

Figure S64: Kinetic profiles for the $C$-3-arylation of indole and $(3-2 \mathrm{H})$-indole at low conversions. No kinetic isotopic effect is observed. 


\subsection{Intermolecular competition experiment}

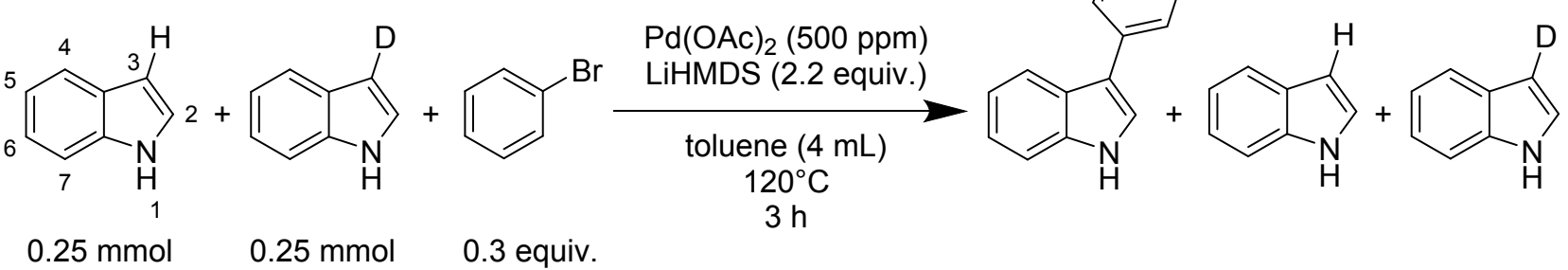

A 1:1 mixture of indole and $(3-2 \mathrm{H})$-indole ( $96 \%$ deuterium incorporation) was prepared. The mixture $(0.5 \mathrm{mmol})$ was then added to a flame-dried Schlenk tube equipped with a magnetic stir bar in a glove box. The flask was sealed with a silicon septum and transferred out of the glove box. Dry toluene $(2.9 \mathrm{~mL})$ and a solution of LiHMDS in toluene (1 M, 1.1 mL, 2.2 equiv.) were injected through the septum and the mixture was stirred for two minutes. Then a solution of $\mathrm{Pd}(\mathrm{OAc})_{2}$ in toluene $(500 \mathrm{ppm}, 50 \mu \mathrm{L}$ of stock solution 1$)$ was injected through the septum along with bromobenzene $(0.15 \mathrm{mmol}, 16 \mu \mathrm{L}, 0.3$ equiv. $)$. The reaction was stirred at $120^{\circ} \mathrm{C}$. After $3 \mathrm{~h}$ the reaction mixture was quenched with $2 \mathrm{~mL}$ of methanol. After removal of the solvent by rotary evaporation the crude mixture was dissolved in $\mathrm{CDCl}_{3}$ and analyzed by NMR.

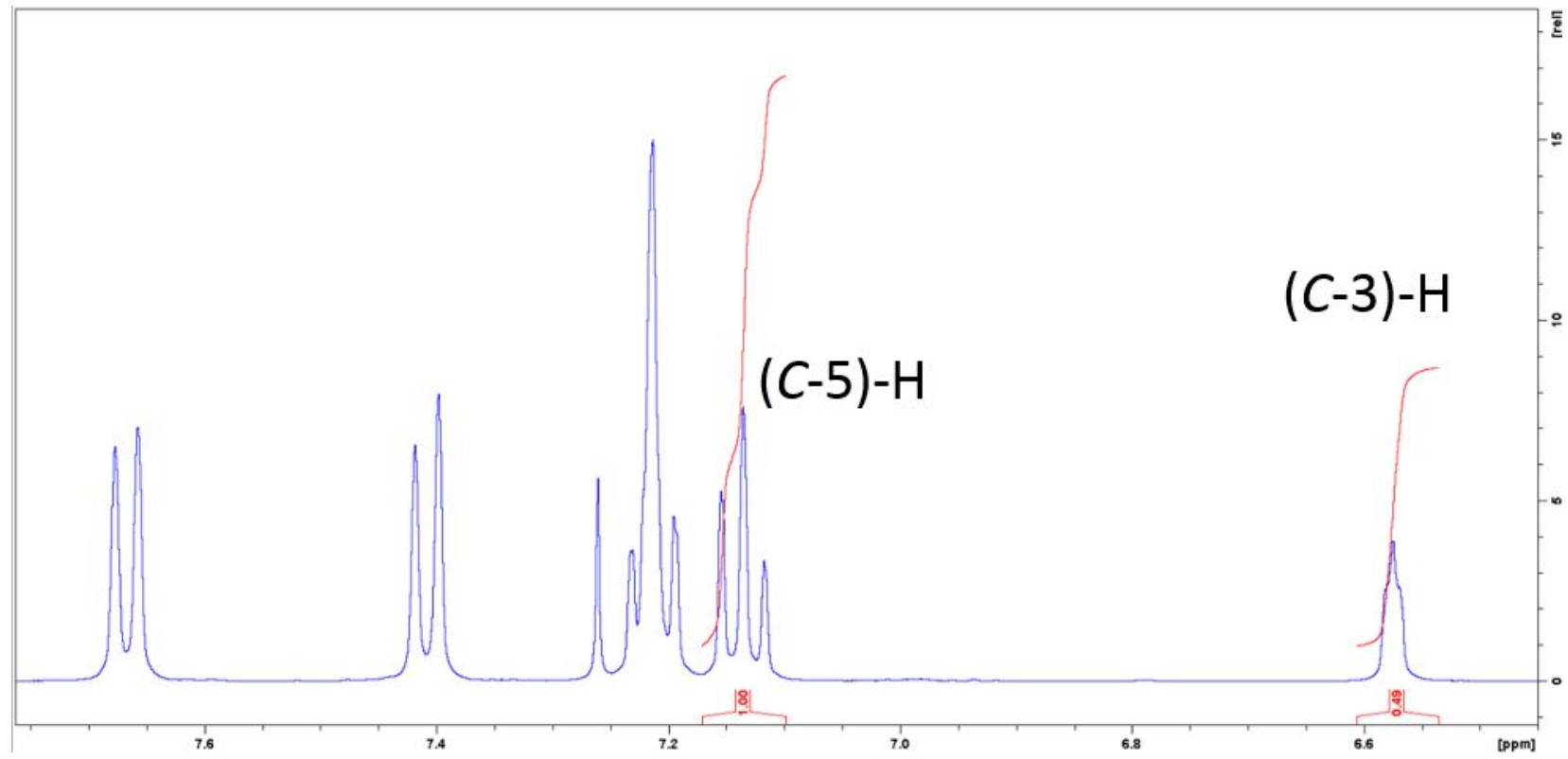

Figure S65: Aromatic section of the ${ }^{1} \mathrm{H}$ NMR spectrum of a mixture of indole and $\left(3-{ }^{2} \mathrm{H}\right)$-indole $\left(400.13 \mathrm{MHz} \mathrm{CDCl}_{3}\right)$. 


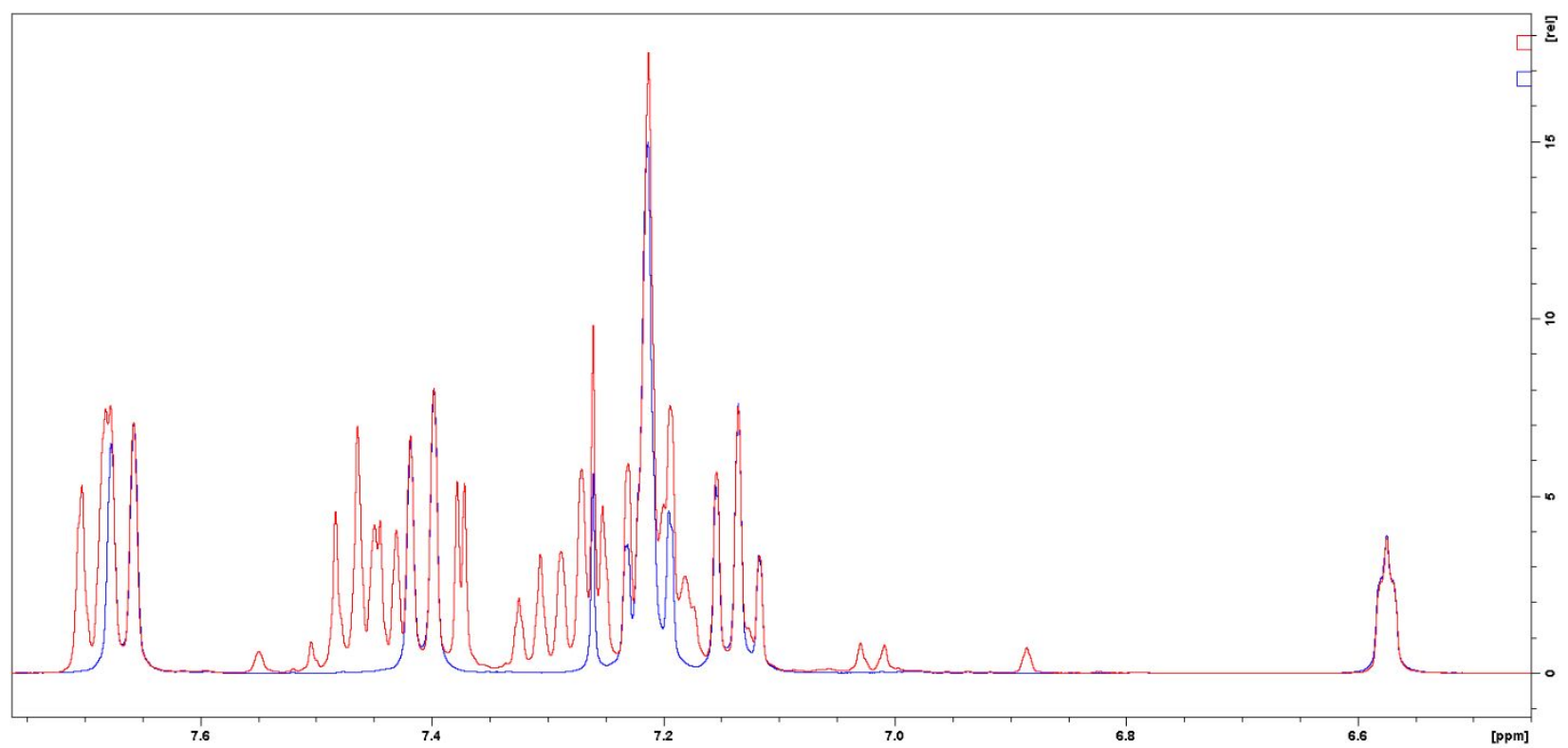

Figure S66: ${ }^{1} \mathrm{H}$ NMR spectra of a (C-3)-H/D-indole mixture before (blue) and after catalysis (red) $(400.13 \mathrm{MHz}, \mathrm{CDCl} 3)$. No change in the (C-5)-H : (C-3)-H signal ratio is observed. Therefore, no kinetic isotopic effect is present. 


\section{Computational details}

Geometry optimizations were performed using Gaussian 16 (Revision A03) ${ }^{8}$ at the PBE0 level of hybrid density functional theory, ${ }^{9}$ with inclusion of D3(bj) corrections in the optimization process. ${ }^{10,11}$ The geometry of all optimized structures is given below. The atoms $\mathrm{C}, \mathrm{H}, \mathrm{N}, \mathrm{Li}$ and $\mathrm{Br}$ were represented by an svp basis set. ${ }^{12}$ The $\mathrm{Pd}$ atom was represented by Dolg's pseudo potential and the associated basis set. ${ }^{13,14}$ The influence of the solvent (toluene) was taken into consideration through single-point calculations on the gas-phase optimized geometries with SCRF calculations within the SMD model. ${ }^{15}$ For the SCRF calculations, the atoms were treated with a def2-qzvp basis set. ${ }^{16}$ All energies reported are Gibbs free energies obtained by summing the SMD energy (including D3 corrections) and the gas phase Gibbs contribution at $393 \mathrm{~K}$ and $1 \mathrm{~atm}$. Starting, LiHMDS is considered as an isolated monomer for simplicity. ${ }^{17}$

The indole deprotonation by LiHMDS here is computed to be easy ( $\Delta \mathrm{G}^{\#}=8.7 \mathrm{kcal} / \mathrm{mol}$ ) and can be described as a metathesis event exchanging $\mathrm{H}$ and $\mathrm{Li}$ between the two nitrogen atoms (see TS-A in Figure S59). The resulting adduct A (Figure S67) is computed to be more stable than separated indole and LiHMDS by $\Delta G=5.3 \mathrm{kcal} / \mathrm{mol}$. Exchange of HHMDS by a further equivalent of LiHMDS is strongly exoergic $(\Delta \mathrm{G}=-25.7 \mathrm{kcal} / \mathrm{mol})$ and leads to the [indolide $\cdot \mathrm{Li}_{2} \cdot \mathrm{HMDS}$ ] intermediate B (Figure S67). Such an intermediate presents a HMDS moiety interacting with indolide through two bridging Li cations.

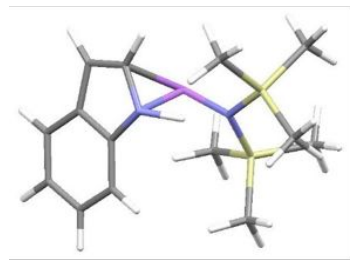

TS-A

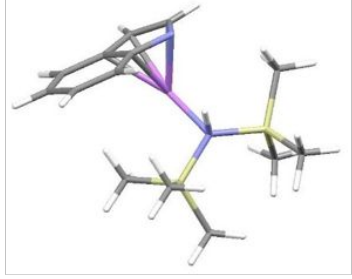

A

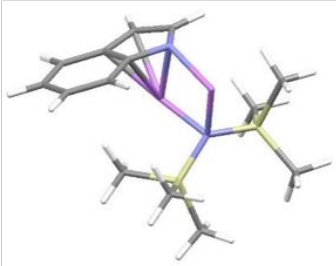

B

Figure S67: Species generated by interaction between indole and one equivalent (TS-A and A) or two equivalent LiHMDS (B). Color code: C: grey, $\mathrm{H}$ : white, N: light blue, Li: purple, Si: yellow.

Coordination of $\mathrm{Pd}(\mathrm{Ph})(\mathrm{Br})$, as a prototype of the product of $\mathrm{Ar}-\mathrm{Br}$ oxidative addition to a $\mathrm{Pd}(0)$ center, leads to two different adducts $\mathbf{C 2}$ and $\mathbf{C} 3$ (Figure S68). $\mathbf{C 2}$ is computed to be $\Delta \mathbf{G}=-11.1$ $\mathrm{kcal} / \mathrm{mol}$ more stable than $\mathbf{B}$. The main driving force is the coordination of the $C-2=C-3$ bond of the 5-membered ring to Pd. The other adduct, C3 (Figure S68), is computed to be even more stable with $\Delta \mathrm{G}=-19.5 \mathrm{kcal} / \mathrm{mol}$ with respect to $\mathrm{B}$. In addition to the coordination of $C-2=C-3$ to $P d$, there is a strong interaction developing between one $\mathrm{Li}$ cation and the bromide on $\mathrm{Pd}(\mathrm{Li} \cdots \mathrm{Br}=$ $2.395 \AA$ ), explaining the increased stability of $\mathbf{C} 3$ with respect to $\mathbf{C 2}$. 


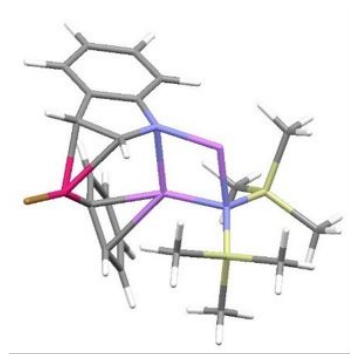

C2

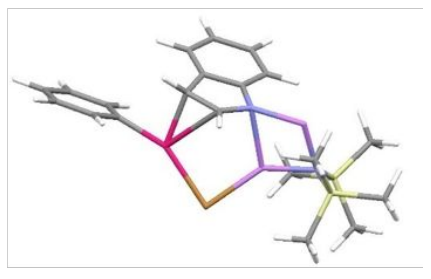

C3

Figure S68: Two possible insertion pathways of $\mathrm{Ar}-\mathrm{Pd}-\mathrm{Br}$ into indolide species (C2 and C3). Color code: C: grey, H: white, N: light blue, Li: purple, Si: yellow, Pd: red, Br: brown.

The transition states for insertion of $C-2=C-3$ into the Pd-Ph bond from $\mathbf{C 2}$ and $\mathbf{C} 3$ were located. TS-C2 (Figure S69) is associated to the formation of the new C-C bond at the C-2 position and lies at $\Delta \mathrm{G}^{\#}=11.1 \mathrm{kcal} / \mathrm{mol}$ above $\mathbf{C 2}$. TS-C3 (Figure S69) is associated to the formation of the new $\mathrm{C}-\mathrm{C}$ bond at the $C-3$ position and lies at $\Delta \mathrm{G}^{\#}=10.2 \mathrm{kcal} / \mathrm{mol}$ above $\mathbf{C} 3$. There is thus a clear kinetic preference for the functionalization at the $C-3$ position. There is also a clear thermodynamic preference for the functionalization at $C-3$ as the product of the $C-2=C-3$ insertion into Pd-Ph, D3 (Figure S69), lies at $\Delta G=-11.4 \mathrm{kcal} / \mathrm{mol}$ below $\mathbf{C 3}$, whereas the corresponding product for the functionalization at C-2, D2 (Figure S69), lies only at $\Delta \mathrm{G}=-0.5 \mathrm{kcal} / \mathrm{mol}$ below $\mathbf{C 2}$.

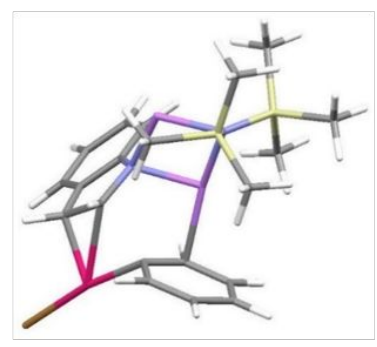

TS-C2

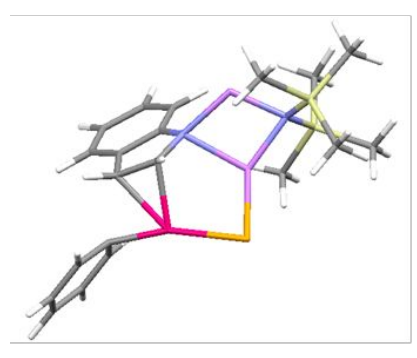

TS-C3

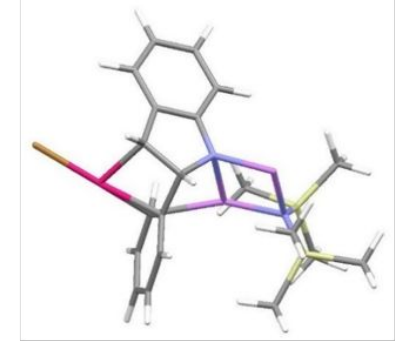

D2

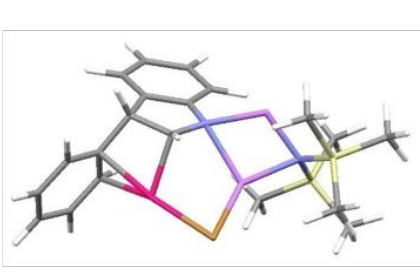

D3

Figure S69: Transition states (TS-C2 and TS-C3) and reaction intermediates (D2 and D3) for the insertion step. Color code: C: grey, H: white, N: light blue, Li: purple, Si: yellow, Pd: red, Br: brown. 


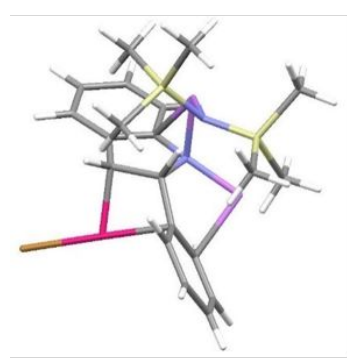

TS-D2

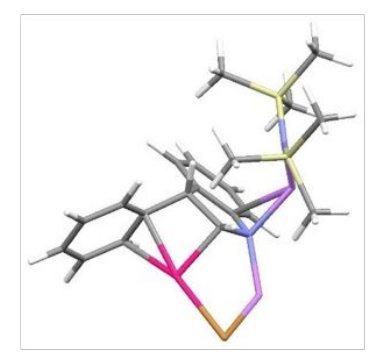

D3'

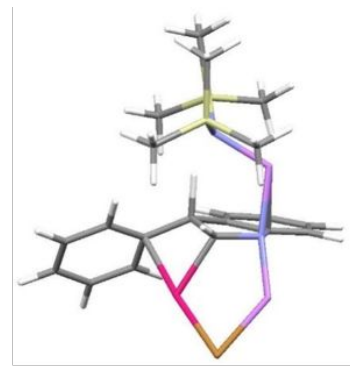

TS-D3

Figure S70: Transition states (TS-D2 and TS-D3) and additional intermediate (D3') for the base-assisted elimination step. Color code: C: grey, $\mathrm{H}$ : white, N: light blue, Li: purple, Si: yellow, Pd: red, Br: brown.

The transition state for deprotonation at the C-2 position (resp. C-3 position) in D2 (resp. D3) have been located (TS-D2 and TS-D3, respectively; Figure S70). Base-assisted elimination in the C-2 position is effective through TS-D2 with an energy barrier of $\Delta \mathrm{G}^{\#}=33.9 \mathrm{kcal} / \mathrm{mol}$ with respect to D2. In the case of base-assisted elimination in the $C$-3 position, LiHMDS has to dissociate in D3 to form D3' (Figure S70) lying at $\Delta \mathrm{G}=-0.5 \mathrm{kcal} / \mathrm{mol}$ below D2. From D3', the actual process of base-assisted elimination at $C$ - 3 is effective through TS-D3 with an activation barrier of $\Delta \mathrm{G}^{\#}=15.5$ $\mathrm{kcal} / \mathrm{mol}$. The base-assisted elimination is thus much easier in the $C-3$ position than in the $C-2$ one. However, isomerization from $\mathbf{D} 3$ to $\mathbf{D} 3$ ' requires an energy of approximately $\Delta \mathrm{G}=18.8$ $\mathrm{kcal} / \mathrm{mol}$.

Alternatively, the possibility to create first a second $\mathrm{Pd}-\mathrm{C}$ bonds $(\mathrm{Pd}-(\mathrm{C}-2)$ or $\mathrm{Pd}-(\mathrm{C}-3))$ in addition to the $\mathrm{Pd}-\mathrm{Ph}$ bond via an electrophilic aromatic substitution $\left(\mathrm{S}_{\mathrm{E}} \mathrm{Ar}\right)$ mechanism and then stepwise deprotonation of the $C-2$ or $C-3$ position followed by reductive elimination was also considered. A concerted metalation-deprotonation (CMD) mechanism was ruled out as no kinetic isotopic effect was observed (see also Section 7). The TS for the formation of the Pd- $(C-2)$ bond, TS-E2 (Figure S71), was located at $\Delta G^{\#}=37.2 \mathrm{kcal} / \mathrm{mol}$ above $\mathbf{C 2}$. This corresponds to a value of $\Delta \Delta \mathrm{G}^{\#}=28.9$ $\mathrm{kcal} / \mathrm{mol}$ with respect to TS-C2, the insertion TS, in favor of the latter. For the formation of the Pd$(C-3)$ bond, decoordination of LiHMDS from C3 to afford the geometry C3' (Figure S71) requires $\Delta G=27.5 \mathrm{kcal} / \mathrm{mol}$. The actual formation of the $\mathrm{Pd}-(C-3)$ bond is effective through TS-E3 (Figure S71) with $\Delta \mathrm{G}^{\#}=18.5 \mathrm{kcal} / \mathrm{mol}$, leading to an overall activation barrier of $\Delta \mathrm{G}^{\#}=46.0$ $\mathrm{kcal} / \mathrm{mol}$, much higher than the insertion barrier of $10.2 \mathrm{kcal} / \mathrm{mol}$.

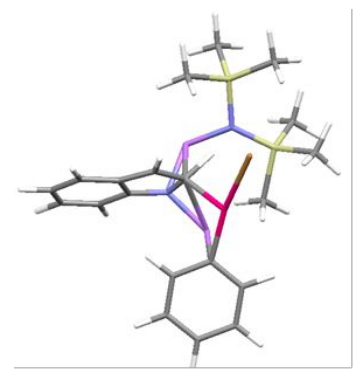

TS-E2

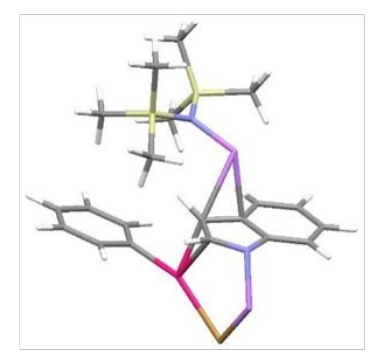

C3'

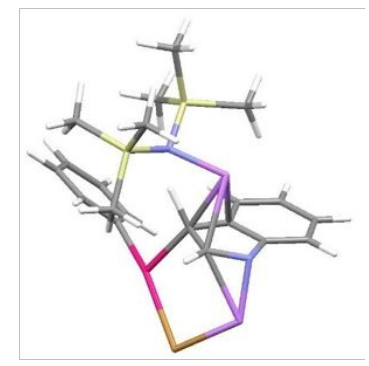

TS-E3

Figure S71: Transition states (TS-E2 and TS-E3) and additional intermediate (C3') for the beginning of a $S_{E} A r$ mechanism. Color code: C: grey, $\mathrm{H}$ : white, N: light blue, Li: purple, Si: yellow, Pd: red, Br: brown. 
The free energy profiles of the two mechanisms are summarized in the Figure S64 showing that a Heck-type mechanism through TS-C species (intermediates in red and blue) is much more favored than a $S_{E} A r$ mechanism through TS-E species (intermediates in green and orange).

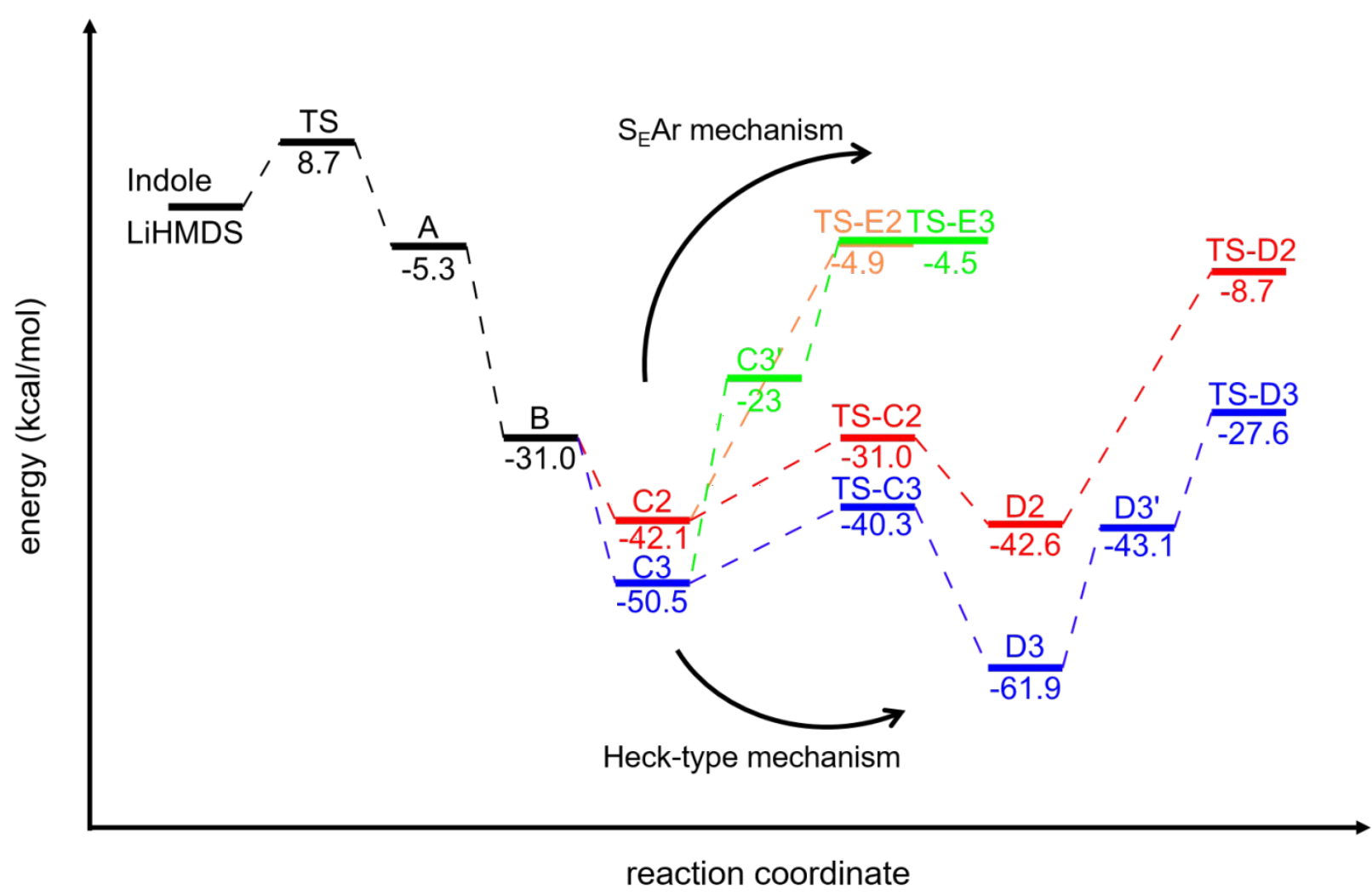

Figure S72: Comparison of free energy profiles for the reported reaction. Heck-type mechanism is shown in red and blue. The beginning of a $S_{E} A r$ mechanism is shown in green and orange. 
Geometry of all optimized structures:

16

Indole

$\begin{array}{llll}\text { C } & 0.25143 & -0.67049 & -0.00001\end{array}$

$\begin{array}{llll}\text { C } & 0.24998 & 0.75158 & 0.00020\end{array}$

$\begin{array}{llll}\text { C } & -0.98183 & 1.42747 & 0.00000\end{array}$

$\begin{array}{llll}\text { C } & -2.15568 & 0.68952 & -0.00012\end{array}$

$\begin{array}{llll}\text { C } & -2.13038 & -0.71898 & -0.00004\end{array}$

$\begin{array}{llll}\text { C } & -0.93109 & -1.41702 & 0.00007\end{array}$

$\begin{array}{llll}\text { C } & 2.38249 & 0.02570 & -0.00007\end{array}$

$\begin{array}{llll}\text { C } & 1.62261 & 1.16671 & 0.00012\end{array}$

$\begin{array}{llll}\text { H } & -1.01121 & 2.51980 & -0.00004\end{array}$

$\begin{array}{llll}\text { H } & -3.11886 & 1.20506 & -0.00024\end{array}$

$\begin{array}{llll}H & -3.07247 & -1.27221 & 0.00010\end{array}$

$\begin{array}{llll}\text { H } & -0.91341 & -2.50947 & 0.00043\end{array}$

$\begin{array}{llll}H & 1.99987 & 2.18679 & 0.00007\end{array}$

$\begin{array}{llll}N & 1.56171 & -1.07593 & -0.00020\end{array}$

$\begin{array}{llll}\text { H } & 3.46482 & -0.09006 & -0.00031\end{array}$

$\begin{array}{llll}H & 1.87413 & -2.03534 & 0.00048\end{array}$

28

LiHMDS

Li $\quad 0.61943 \quad-2.38589 \quad-0.00602$

$\begin{array}{llll}N & -0.01271 & -0.71583 & -0.02535\end{array}$

$\begin{array}{llll}\text { Si } & -1.58799 & -0.04612 & -0.00685\end{array}$

$\begin{array}{llll}\text { Si } & 1.51442 & 0.03337 & -0.00188\end{array}$

$\begin{array}{llll}\text { C } & -2.04958 & 0.67218 & 1.68290\end{array}$

$\begin{array}{llll}H & -1.37430 & 1.50018 & 1.95329\end{array}$

$\begin{array}{llll}H & -3.08138 & 1.06009 & 1.69899\end{array}$

$\begin{array}{llll}H & -1.96117 & -0.09431 & 2.46990\end{array}$ 


\begin{tabular}{llll}
$\mathrm{C}$ & -2.84065 & -1.41221 & -0.39892 \\
$\mathrm{H}$ & -3.87612 & -1.03532 & -0.38709 \\
$\mathrm{H}$ & -2.65394 & -1.84305 & -1.39674 \\
$\mathrm{H}$ & -2.78114 & -2.22815 & 0.34178 \\
$\mathrm{C}$ & -1.82506 & 1.33370 & -1.28064 \\
$\mathrm{H}$ & -1.55273 & 0.98796 & -2.29079 \\
$\mathrm{H}$ & -2.86875 & 1.68711 & -1.30922 \\
$\mathrm{H}$ & -1.18761 & 2.20150 & -1.04371 \\
$\mathrm{C}$ & 1.97362 & 1.01640 & -1.54632 \\
$\mathrm{H}$ & 1.83978 & 0.40657 & -2.45387 \\
$\mathrm{H}$ & 1.31985 & 1.89754 & -1.64553 \\
$\mathrm{H}$ & 3.01635 & 1.37237 & -1.51662 \\
$\mathrm{C}$ & 1.88355 & 1.11026 & 1.50354 \\
$\mathrm{H}$ & 1.67252 & 0.56689 & 2.43807 \\
$\mathrm{H}$ & 2.93132 & 1.45148 & 1.52602 \\
$\mathrm{H}$ & 1.24128 & 2.00603 & 1.49286 \\
$\mathrm{C}$ & 2.75876 & -1.44553 & 0.07032 \\
$\mathrm{H}$ & 2.71298 & -2.10990 & -0.81689 \\
$\mathrm{H}$ & 3.79353 & -1.06997 & 0.08280 \\
$\mathrm{H}$ & 2.66636 & -2.05869 & 0.98928 \\
\hline & & & \\
\hline & & & \\
\hline
\end{tabular}

\section{TS-A}

$\begin{array}{llll}\text { C } & 2.19258 & -0.50187 & 0.51202 \\ \text { C } & 3.14506 & -1.03949 & -0.40419 \\ \text { C } & 4.39901 & -0.41571 & -0.54542 \\ \text { C } & 4.66980 & 0.71643 & 0.20433 \\ \text { C } & 3.71730 & 1.24103 & 1.10513 \\ \text { C } & 2.47934 & 0.64293 & 1.27286 \\ \text { C } & 1.25166 & -2.25841 & -0.43806 \\ \text { C } & 2.50930 & -2.17207 & -1.00914\end{array}$




\begin{tabular}{|c|c|c|c|}
\hline $\mathrm{H}$ & 5.14544 & -0.81973 & -1.23386 \\
\hline $\mathrm{H}$ & 5.63870 & 1.21157 & 0.10614 \\
\hline $\mathrm{H}$ & 3.96819 & 2.12937 & 1.68970 \\
\hline$\vdash$ & 1.75021 & 1.03365 & 1.98472 \\
\hline $\mathrm{H}$ & 2.93978 & -2.86173 & -1.73324 \\
\hline$N$ & 1.03798 & -1.25065 & 0.47822 \\
\hline $\mathrm{H}$ & 0.47648 & -3.00482 & -0.61504 \\
\hline $\mathrm{Li}$ & 0.35015 & -0.36447 & -1.34497 \\
\hline$N$ & -1.15463 & -0.07770 & -0.13964 \\
\hline $\mathrm{Si}$ & -1.13245 & 1.64250 & -0.35433 \\
\hline $\mathrm{Si}$ & -2.58960 & -0.97581 & 0.26652 \\
\hline C & 0.37920 & 1.99992 & -1.47426 \\
\hline $\mathrm{H}$ & 0.27978 & 1.56397 & -2.48891 \\
\hline $\mathrm{H}$ & 0.46625 & 3.08445 & -1.64264 \\
\hline $\mathrm{H}$ & 1.34622 & 1.69142 & -1.03551 \\
\hline C & -0.92487 & 2.62871 & 1.23435 \\
\hline $\mathrm{H}$ & -0.86644 & 3.71015 & 1.03147 \\
\hline $\mathrm{H}$ & -1.78285 & 2.45447 & 1.90243 \\
\hline $\mathrm{H}$ & -0.01425 & 2.33529 & 1.77683 \\
\hline C & -2.63560 & 2.31600 & -1.27000 \\
\hline $\mathrm{H}$ & -3.55882 & 2.17900 & -0.68538 \\
\hline $\mathrm{H}$ & -2.52018 & 3.39570 & -1.45812 \\
\hline $\mathrm{H}$ & -2.77510 & 1.81313 & -2.23959 \\
\hline C & -2.09932 & -2.71874 & 0.79803 \\
\hline $\mathrm{H}$ & -1.73068 & -3.31340 & -0.05333 \\
\hline $\mathrm{H}$ & -1.31475 & -2.70564 & 1.57079 \\
\hline $\mathrm{H}$ & -2.97211 & -3.24995 & 1.21017 \\
\hline C & -3.53382 & -0.17497 & 1.69287 \\
\hline H & -3.88884 & 0.83469 & 1.43033 \\
\hline $\mathrm{H}$ & -4.41570 & -0.77253 & 1.97469 \\
\hline
\end{tabular}




$\begin{array}{llll}\mathrm{H} & -2.88876 & -0.08217 & 2.58121 \\ \mathrm{C} & -3.76809 & -1.14676 & -1.19911 \\ \mathrm{H} & -3.25509 & -1.60439 & -2.06078 \\ \mathrm{H} & -4.62569 & -1.78904 & -0.94020 \\ \mathrm{H} & -4.16497 & -0.17288 & -1.52346 \\ \mathrm{H} & -0.05123 & -0.70437 & 0.46930 \\ \mathrm{H} & & & \end{array}$

A

$\begin{array}{llll}\text { C } & 2.20066 & -0.86906 & 0.70560\end{array}$

$\begin{array}{llll}\text { C } & 2.82612 & -0.93094 & -0.59075\end{array}$

$\begin{array}{llll}\text { C } & 3.70654 & 0.09973 & -0.99251\end{array}$

C $\quad 3.93504 \quad 1.15676 \quad-0.13206$

$\begin{array}{llll}\text { C } & 3.30374 & 1.22464 & 1.13541\end{array}$

C $\quad 2.44254 \quad 0.23065 \quad 1.55965$

$\begin{array}{llll}\text { C } & 1.50584 & -2.70762 & -0.19655\end{array}$

$\begin{array}{llll}\text { C } & 2.33921 & -2.13963 & -1.17507\end{array}$

H $4.20334 \quad 0.05826 \quad-1.96550$

$\begin{array}{llll}\mathrm{H} & 4.61732 & 1.95721 & -0.42873\end{array}$

$\begin{array}{llll}\mathrm{H} & 3.51408 & 2.07468 & 1.78941\end{array}$

$\begin{array}{llll}H & 1.97325 & 0.26304 & 2.54651\end{array}$

$\begin{array}{llll}\mathrm{H} & 2.61499 & -2.57048 & -2.13656\end{array}$

$\begin{array}{llll}N & 1.37630 & -1.93962 & 0.91564\end{array}$

$\begin{array}{llll}H & 0.96737 & -3.65544 & -0.27486\end{array}$

Li $\quad 0.55514 \quad-0.86399 \quad-0.69385$

$\begin{array}{llll}N & -1.06959 & 0.04252 & 0.12342\end{array}$

Si $\quad-0.92218 \quad 1.79111 \quad-0.21734$

Si $\quad-2.55500 \quad-0.94485 \quad 0.12039$

$\begin{array}{llll}\text { C } & 0.51628 & 2.02740 & -1.39791\end{array}$

$\begin{array}{llll}\mathrm{H} & 0.34881 & 1.49116 & -2.34755\end{array}$

$\begin{array}{llll}\mathrm{H} & 0.63822 & 3.09352 & -1.64804\end{array}$ 


\begin{tabular}{|c|c|c|c|}
\hline $\mathrm{H}$ & 1.47276 & 1.68807 & -0.96655 \\
\hline C & -0.59895 & 2.67493 & 1.40223 \\
\hline $\mathrm{H}$ & -0.47435 & 3.75741 & 1.24125 \\
\hline $\mathrm{H}$ & -1.42963 & 2.52834 & 2.11039 \\
\hline $\mathrm{H}$ & 0.32602 & 2.29746 & 1.86556 \\
\hline C & -2.47832 & 2.45358 & -1.03166 \\
\hline $\mathrm{H}$ & -3.38193 & 2.28954 & -0.42567 \\
\hline $\mathrm{H}$ & -2.36396 & 3.54092 & -1.16922 \\
\hline $\mathrm{H}$ & -2.64155 & 2.01212 & -2.02610 \\
\hline C & -2.04290 & -2.69183 & 0.56323 \\
\hline $\mathrm{H}$ & -1.54875 & -3.18727 & -0.28714 \\
\hline $\mathrm{H}$ & -1.32896 & -2.69950 & 1.40197 \\
\hline $\mathrm{H}$ & -2.92199 & -3.29543 & 0.83892 \\
\hline C & -3.77431 & -0.29857 & 1.39622 \\
\hline $\mathrm{H}$ & -4.08147 & 0.73751 & 1.18641 \\
\hline $\mathrm{H}$ & -4.68380 & -0.91999 & 1.42024 \\
\hline $\mathrm{H}$ & -3.32818 & -0.32115 & 2.40342 \\
\hline C & -3.31597 & -0.92055 & -1.59478 \\
\hline $\mathrm{H}$ & -2.56831 & -1.16400 & -2.36645 \\
\hline $\mathrm{H}$ & -4.10814 & -1.68445 & -1.65011 \\
\hline$\vdash$ & -3.77001 & 0.04774 & -1.84807 \\
\hline $\mathrm{H}$ & -0.57002 & -0.17206 & 0.99565 \\
\hline 44 & & & \\
\hline B & & & \\
\hline C & -2.22624 & -0.79782 & -0.70842 \\
\hline C & -2.75146 & -1.07355 & 0.59755 \\
\hline C & -3.75897 & -0.24261 & 1.13405 \\
\hline C & -4.20965 & 0.82872 & 0.38571 \\
\hline$C$ & -3.68359 & 1.09963 & -0.89971 \\
\hline$C$ & -2.70078 & 0.30098 & -1.45409 \\
\hline
\end{tabular}




\begin{tabular}{|c|c|c|c|}
\hline C & -1.15539 & -2.54763 & 0.02167 \\
\hline$C$ & -2.02688 & -2.21648 & 1.06003 \\
\hline $\mathrm{H}$ & -4.17540 & -0.44455 & 2.12412 \\
\hline $\mathrm{H}$ & -4.98857 & 1.48014 & 0.78880 \\
\hline $\mathrm{H}$ & -4.06718 & 1.95309 & -1.46366 \\
\hline $\mathrm{H}$ & -2.31121 & 0.50092 & -2.45611 \\
\hline $\mathrm{H}$ & -2.16567 & -2.75771 & 1.99446 \\
\hline$N$ & -1.23512 & -1.68930 & -1.03604 \\
\hline $\mathrm{H}$ & -0.44410 & -3.37651 & 0.00843 \\
\hline $\mathrm{Li}$ & 0.40278 & -0.83932 & -1.65004 \\
\hline $\mathrm{Li}$ & -0.47398 & -0.51718 & 0.73900 \\
\hline$N$ & 1.15583 & 0.13370 & -0.15276 \\
\hline Si & 0.93006 & 1.81726 & 0.14758 \\
\hline Si & 2.55987 & -0.84805 & -0.01667 \\
\hline C & -0.56293 & 2.00090 & 1.31259 \\
\hline $\mathrm{H}$ & -0.39070 & 1.50479 & 2.28539 \\
\hline $\mathrm{H}$ & -0.74776 & 3.06341 & 1.53736 \\
\hline $\mathrm{H}$ & -1.50587 & 1.61864 & 0.88181 \\
\hline C & 0.50768 & 2.74574 & -1.44140 \\
\hline $\mathrm{H}$ & 0.32215 & 3.81599 & -1.25600 \\
\hline $\mathrm{H}$ & 1.33109 & 2.66822 & -2.17032 \\
\hline $\mathrm{H}$ & -0.40231 & 2.32890 & -1.90353 \\
\hline C & 2.37784 & 2.69625 & 0.97832 \\
\hline $\mathrm{H}$ & 3.25462 & 2.75351 & 0.31550 \\
\hline $\mathrm{H}$ & 2.09316 & 3.72658 & 1.24657 \\
\hline $\mathrm{H}$ & 2.68470 & 2.18119 & 1.90290 \\
\hline C & 2.44008 & -2.15397 & -1.41271 \\
\hline $\mathrm{H}$ & 1.52304 & -2.77031 & -1.37616 \\
\hline $\mathrm{H}$ & 2.52264 & -1.70118 & -2.41918 \\
\hline $\mathrm{H}$ & 3.27653 & -2.86624 & -1.33811 \\
\hline
\end{tabular}




\begin{tabular}{|c|c|c|c|}
\hline$C$ & 4.21931 & 0.01155 & -0.26031 \\
\hline $\mathrm{H}$ & 4.45936 & 0.67597 & 0.58324 \\
\hline $\mathrm{H}$ & 5.02837 & -0.73251 & -0.34093 \\
\hline $\mathrm{H}$ & 4.22344 & 0.61881 & -1.17954 \\
\hline C & 2.61719 & -1.79784 & 1.61468 \\
\hline $\mathrm{H}$ & 1.69822 & -2.38980 & 1.76135 \\
\hline $\mathrm{H}$ & 3.47354 & -2.48929 & 1.66730 \\
\hline $\mathrm{H}$ & 2.70022 & -1.09548 & 2.46043 \\
\hline 7 & & & \\
\hline 2 & & & \\
\hline$C$ & 0.44681 & 2.08882 & -1.39391 \\
\hline$C$ & 1.83831 & 2.14512 & -1.12450 \\
\hline$C$ & 2.44799 & 3.35815 & -0.79320 \\
\hline$C$ & 1.65761 & 4.50099 & -0.71424 \\
\hline$C$ & 0.27592 & 4.43698 & -0.95971 \\
\hline C & -0.34452 & 3.23919 & -1.29922 \\
\hline C & 1.16294 & 0.03906 & -1.66025 \\
\hline$C$ & 2.32664 & 0.78966 & -1.25421 \\
\hline $\mathrm{H}$ & 3.51974 & 3.40233 & -0.58623 \\
\hline $\mathrm{H}$ & 2.11321 & 5.45951 & -0.45647 \\
\hline $\mathrm{H}$ & -0.32317 & 5.34767 & -0.88669 \\
\hline $\mathrm{H}$ & -1.41915 & 3.20403 & -1.48955 \\
\hline $\mathrm{H}$ & 3.34804 & 0.52150 & -1.53525 \\
\hline $\mathrm{N}$ & 0.04769 & 0.79502 & -1.71853 \\
\hline $\mathrm{H}$ & 1.17506 & -0.98957 & -2.02890 \\
\hline Li & -0.83709 & 0.10867 & 0.14663 \\
\hline $\mathrm{Li}$ & -1.70389 & 0.05024 & -2.18836 \\
\hline $\mathrm{N}$ & -2.48213 & -0.63370 & -0.58081 \\
\hline $\mathrm{Si}$ & -3.77969 & 0.49223 & -0.44047 \\
\hline SI & -2.52325 & -2.33869 & -0.29382 \\
\hline
\end{tabular}




\begin{tabular}{|c|c|c|c|}
\hline C & -3.89622 & 1.40963 & -2.11547 \\
\hline $\mathrm{H}$ & -4.22427 & 0.72676 & -2.91914 \\
\hline $\mathrm{H}$ & -4.63642 & 2.22454 & -2.07300 \\
\hline $\mathrm{H}$ & -2.95515 & 1.89640 & -2.43728 \\
\hline C & -3.40090 & 1.79008 & 0.87854 \\
\hline $\mathrm{H}$ & -4.16812 & 2.58049 & 0.90919 \\
\hline $\mathrm{H}$ & -3.36019 & 1.31973 & 1.87427 \\
\hline $\mathrm{H}$ & -2.42857 & 2.28540 & 0.71384 \\
\hline C & -5.48797 & -0.21924 & -0.09715 \\
\hline $\mathrm{H}$ & -5.50440 & -0.77542 & 0.85313 \\
\hline $\mathrm{H}$ & -6.23421 & 0.58821 & -0.02439 \\
\hline $\mathrm{H}$ & -5.80902 & -0.90520 & -0.89589 \\
\hline C & -0.79586 & -3.02796 & -0.64772 \\
\hline $\mathrm{H}$ & -0.53150 & -2.92717 & -1.71423 \\
\hline $\mathrm{H}$ & 0.00640 & -2.53576 & -0.07097 \\
\hline $\mathrm{H}$ & -0.74175 & -4.10201 & -0.40875 \\
\hline C & -2.97690 & -2.77675 & 1.48635 \\
\hline $\mathrm{H}$ & -4.00030 & -2.44783 & 1.72694 \\
\hline $\mathrm{H}$ & -2.92564 & -3.86384 & 1.65879 \\
\hline $\mathrm{H}$ & -2.29539 & -2.28657 & 2.19881 \\
\hline C & -3.71791 & -3.25693 & -1.43424 \\
\hline $\mathrm{H}$ & -3.50143 & -3.04234 & -2.49383 \\
\hline $\mathrm{H}$ & -3.64148 & -4.34725 & -1.29302 \\
\hline $\mathrm{H}$ & -4.76284 & -2.96883 & -1.24071 \\
\hline $\mathrm{Pd}$ & 2.16116 & -0.50980 & 0.42763 \\
\hline $\mathrm{Br}$ & 3.73542 & -2.16722 & -0.48483 \\
\hline C & 0.88516 & 0.46469 & 1.59559 \\
\hline C & 0.71749 & 1.83416 & 1.84717 \\
\hline C & 0.15783 & -0.45103 & 2.38309 \\
\hline C & -0.14167 & 2.26861 & 2.85964 \\
\hline
\end{tabular}




\begin{tabular}{|c|c|c|c|}
\hline $\mathrm{H}$ & 1.27033 & 2.57542 & 1.26740 \\
\hline C & -0.70569 & -0.01191 & 3.39264 \\
\hline $\mathrm{H}$ & 0.26914 & -1.53222 & 2.22537 \\
\hline C & -0.85672 & 1.35210 & 3.63024 \\
\hline $\mathrm{H}$ & -0.24942 & 3.34019 & 3.04621 \\
\hline $\mathrm{H}$ & -1.25933 & -0.74228 & 3.98799 \\
\hline $\mathrm{H}$ & -1.53212 & 1.70137 & 4.41445 \\
\hline 7 & & & \\
\hline C3 & & & \\
\hline$C$ & 0.27311 & 1.93669 & -0.96683 \\
\hline C & 1.68003 & 1.78732 & -0.98717 \\
\hline C & 2.49944 & 2.84148 & -0.57871 \\
\hline C & 1.89889 & 4.01374 & -0.12411 \\
\hline C & 0.50313 & 4.14438 & -0.07894 \\
\hline C & -0.32660 & 3.10982 & -0.50348 \\
\hline C & 0.61194 & -0.04713 & -1.81971 \\
\hline C & 1.93406 & 0.43834 & -1.50290 \\
\hline $\mathrm{H}$ & 3.58656 & 2.74578 & -0.61168 \\
\hline $\mathrm{H}$ & 2.52455 & 4.84787 & 0.20174 \\
\hline $\mathrm{H}$ & 0.06043 & 5.07392 & 0.28580 \\
\hline $\mathrm{H}$ & -1.41399 & 3.21967 & -0.48174 \\
\hline $\mathrm{H}$ & 2.79758 & 0.20326 & -2.13001 \\
\hline$N$ & -0.35820 & 0.79466 & -1.47326 \\
\hline $\mathrm{H}$ & 0.38470 & -0.99736 & -2.30893 \\
\hline $\mathrm{Li}$ & -1.40472 & -0.15399 & 0.36324 \\
\hline $\mathrm{Li}$ & -2.28925 & 0.70073 & -1.81156 \\
\hline$N$ & -3.23819 & 0.14113 & -0.27124 \\
\hline $\mathrm{Si}$ & -3.80489 & 1.04385 & 1.08755 \\
\hline $\mathrm{Si}$ & -4.05998 & -1.14581 & -1.06982 \\
\hline$C$ & -4.55487 & 2.69898 & 0.57223 \\
\hline
\end{tabular}




\begin{tabular}{|c|c|c|c|}
\hline $\mathrm{H}$ & -5.43334 & 2.53385 & -0.07267 \\
\hline $\mathrm{H}$ & -4.88034 & 3.29411 & 1.44063 \\
\hline $\mathrm{H}$ & -3.83415 & 3.30803 & 0.00166 \\
\hline C & -2.30229 & 1.39980 & 2.19792 \\
\hline $\mathrm{H}$ & -2.60140 & 2.02772 & 3.05223 \\
\hline $\mathrm{H}$ & -1.87442 & 0.47652 & 2.62937 \\
\hline $\mathrm{H}$ & -1.49217 & 1.94779 & 1.68489 \\
\hline C & -5.06024 & 0.13358 & 2.16134 \\
\hline $\mathrm{H}$ & -4.65734 & -0.83581 & 2.49528 \\
\hline $\mathrm{H}$ & -5.30565 & 0.72319 & 3.05938 \\
\hline $\mathrm{H}$ & -6.00171 & -0.05762 & 1.62348 \\
\hline C & -3.20646 & -1.29332 & -2.78263 \\
\hline $\mathrm{H}$ & -3.33877 & -0.40658 & -3.43467 \\
\hline $\mathrm{H}$ & -2.12781 & -1.52115 & -2.70669 \\
\hline $\mathrm{H}$ & -3.65111 & -2.12655 & -3.34833 \\
\hline C & -3.88110 & -2.81426 & -0.22282 \\
\hline $\mathrm{H}$ & -4.38590 & -2.79917 & 0.75637 \\
\hline $\mathrm{H}$ & -4.32394 & -3.62911 & -0.81784 \\
\hline $\mathrm{H}$ & -2.81961 & -3.04918 & -0.04268 \\
\hline C & -5.88401 & -0.81952 & -1.42495 \\
\hline $\mathrm{H}$ & -6.02150 & 0.14668 & -1.93663 \\
\hline $\mathrm{H}$ & -6.31366 & -1.60821 & -2.06358 \\
\hline $\mathrm{H}$ & -6.47277 & -0.79056 & -0.49504 \\
\hline $\mathrm{Pd}$ & 1.99404 & -0.85005 & 0.18134 \\
\hline $\mathrm{Br}$ & -0.05672 & -1.92102 & 1.25642 \\
\hline C & 3.89591 & -0.53124 & -0.01392 \\
\hline C & 4.69630 & -1.36521 & -0.79924 \\
\hline C & 4.47935 & 0.40817 & 0.84087 \\
\hline C & 6.08626 & -1.29814 & -0.67854 \\
\hline $\mathrm{H}$ & 4.24394 & -2.07822 & -1.49387 \\
\hline
\end{tabular}




$\begin{array}{llll}\text { C } & 5.87016 & 0.46582 & 0.95463 \\ \text { H } & 3.85479 & 1.08940 & 1.42647 \\ \text { C } & 6.67384 & -0.38544 & 0.19663 \\ \text { H } & 6.71257 & -1.96287 & -1.27933 \\ \text { H } & 6.32613 & 1.18736 & 1.63776 \\ \text { H } & 7.76161 & -0.33155 & 0.28230 \\ 57 & & & \end{array}$

\section{TS-C2}

\begin{tabular}{llll} 
C & 0.47651 & 2.01388 & -0.53421 \\
C & 1.86471 & 1.85655 & -0.78732 \\
C & 2.76525 & 2.89243 & -0.52209 \\
C & 2.27329 & 4.08506 & -0.00655 \\
C & 0.89879 & 4.24280 & 0.24523 \\
C & -0.00950 & 3.22111 & -0.00607 \\
C & 0.68135 & -0.05770 & -1.26733 \\
C & 2.03701 & 0.51694 & -1.32969 \\
H & 3.83345 & 2.74198 & -0.69396 \\
H & 2.95823 & 4.90587 & 0.21577 \\
H & 0.53596 & 5.19001 & 0.65226 \\
H & -1.07549 & 3.36155 & 0.19321 \\
H & 2.71288 & 0.30817 & -2.16682 \\
$\mathrm{~N}$ & -0.25146 & 0.89102 & -0.89168 \\
$\mathrm{H}$ & 0.37858 & -0.83519 & -1.97337 \\
$\mathrm{Li}$ & -1.51139 & 0.03250 & 0.52211 \\
$\mathrm{Li}$ & -2.06547 & 0.93719 & -1.61049 \\
$\mathrm{~N}$ & -3.26011 & 0.20162 & -0.31239 \\
$\mathrm{Si}$ & -4.04763 & 1.12694 & 0.91347 \\
$\mathrm{Si}$ & -3.96165 & -1.05120 & -1.26571 \\
$\mathrm{C}$ & -4.64520 & 2.79359 & 0.26041 \\
\hline & -5.39081 & 2.64609 & -0.53777 \\
\hline
\end{tabular}




\begin{tabular}{|c|c|c|c|}
\hline $\mathrm{H}$ & -5.11180 & 3.40640 & 1.04827 \\
\hline $\mathrm{H}$ & -3.81244 & 3.37829 & -0.16560 \\
\hline$C$ & -2.75415 & 1.46963 & 2.27026 \\
\hline $\mathrm{H}$ & -3.16072 & 2.16448 & 3.02200 \\
\hline $\mathrm{H}$ & -2.48449 & 0.54725 & 2.81402 \\
\hline $\mathrm{H}$ & -1.82525 & 1.94044 & 1.89951 \\
\hline$C$ & -5.48902 & 0.25488 & 1.75896 \\
\hline $\mathrm{H}$ & -5.16596 & -0.70230 & 2.19809 \\
\hline $\mathrm{H}$ & -5.90322 & 0.87574 & 2.56956 \\
\hline $\mathrm{H}$ & -6.30760 & 0.04196 & 1.05386 \\
\hline$C$ & -2.75239 & -1.33768 & -2.71582 \\
\hline $\mathrm{H}$ & -2.59186 & -0.45094 & -3.36039 \\
\hline $\mathrm{H}$ & -1.76908 & -1.69062 & -2.36132 \\
\hline $\mathrm{H}$ & -3.14173 & -2.11406 & -3.39319 \\
\hline$C$ & -4.13369 & -2.69286 & -0.35945 \\
\hline $\mathrm{H}$ & -4.84855 & -2.59981 & 0.47341 \\
\hline $\mathrm{H}$ & -4.49426 & -3.49352 & -1.02504 \\
\hline $\mathrm{H}$ & -3.16564 & -3.00506 & 0.06316 \\
\hline C & -5.62865 & -0.59667 & -2.02403 \\
\hline $\mathrm{H}$ & -5.55977 & 0.33939 & -2.60169 \\
\hline $\mathrm{H}$ & -5.99183 & -1.38643 & -2.70127 \\
\hline $\mathrm{H}$ & -6.39493 & -0.44947 & -1.24674 \\
\hline $\mathrm{Pd}$ & 2.70793 & -0.80030 & 0.03593 \\
\hline $\mathrm{Br}$ & 5.11499 & -0.46861 & -0.01535 \\
\hline C & 0.74612 & -1.29699 & 0.29295 \\
\hline C & 0.39595 & -0.75910 & 1.55464 \\
\hline C & 0.30407 & -2.59157 & -0.02933 \\
\hline C & -0.32968 & -1.51981 & 2.47460 \\
\hline $\mathrm{H}$ & 0.71052 & 0.25997 & 1.81160 \\
\hline C & -0.43847 & -3.33821 & 0.886 \\
\hline
\end{tabular}




$\begin{array}{llll}\text { H } & 0.54957 & -3.02067 & -1.00544 \\ \mathrm{C} & -0.75655 & -2.80861 & 2.13755 \\ \mathrm{H} & -0.55618 & -1.10708 & 3.46118 \\ \mathrm{H} & -0.77191 & -4.34416 & 0.61987 \\ \mathrm{H} & -1.33797 & -3.39664 & 2.85068 \\ \mathrm{H} 7 & & & \end{array}$

\section{TS-C3}

\begin{tabular}{|c|c|c|c|}
\hline & 0.58030 & 2.01454 & -0.84954 \\
\hline & 1.97773 & 1.89014 & -1.03644 \\
\hline & 2.83154 & .95777 & 0.76008 \\
\hline & 2.28426 & 4.14601 & -0.28340 \\
\hline & 0.90122 & 4.25964 & -0.07290 \\
\hline & 0.03717 & 3.20463 & -0.34687 \\
\hline & 0.85429 & -0.01979 & -1.63001 \\
\hline & 2.21136 & 0.52635 & -1.51300 \\
\hline & 3.90985 & 2.85799 & -0.90460 \\
\hline & 2.93459 & 4.99571 & -0.06543 \\
\hline & 0.49363 & 5.19671 & 0.31413 \\
\hline & -1.03735 & 3.30421 & -0.17257 \\
\hline & 2.95081 & 0.30687 & -2.28429 \\
\hline & -0.10011 & 0.87053 & -1.24331 \\
\hline & 0.58139 & -0.84943 & -2.29078 \\
\hline & -1.20316 & -0.28308 & 0.31469 \\
\hline & -2.01775 & 0.98144 & -1.61376 \\
\hline & -3.01945 & 0.33441 & -0.14406 \\
\hline & -3.36034 & 1.05579 & 1.38339 \\
\hline r & -4.07805 & -0.64451 & -1.08341 \\
\hline C & -4.00463 & 2.82390 & 1.22216 \\
\hline & -4.95787 & 2.83066 & 0.66900 \\
\hline & -4.18015 & 3.29128 & 2.20456 \\
\hline
\end{tabular}




\begin{tabular}{|c|c|c|c|}
\hline $\mathrm{H}$ & -3.29634 & 3.46233 & 0.66891 \\
\hline C & -1.71690 & 1.11716 & 2.34463 \\
\hline $\mathrm{H}$ & -1.85676 & 1.62795 & 3.31038 \\
\hline $\mathrm{H}$ & -1.33417 & 0.10849 & 2.58871 \\
\hline $\mathrm{H}$ & -0.92394 & 1.67309 & 1.81333 \\
\hline C & -4.57560 & 0.07801 & 2.44407 \\
\hline $\mathrm{H}$ & -4.20914 & -0.94852 & 2.60379 \\
\hline $\mathrm{H}$ & -4.71024 & 0.54768 & 3.43177 \\
\hline $\mathrm{H}$ & -5.56772 & 0.01144 & 1.97009 \\
\hline C & -3.30999 & -0.64775 & -2.84150 \\
\hline $\mathrm{H}$ & -3.30760 & 0.34568 & -3.33347 \\
\hline $\mathrm{H}$ & -2.28182 & -1.05434 & -2.85323 \\
\hline $\mathrm{H}$ & -3.89722 & -1.29395 & -3.51202 \\
\hline C & -4.18311 & -2.43548 & -0.52261 \\
\hline $\mathrm{H}$ & -4.64652 & -2.49489 & 0.47510 \\
\hline $\mathrm{H}$ & -4.78510 & -3.04905 & -1.21209 \\
\hline $\mathrm{H}$ & -3.17644 & -2.87666 & -0.44633 \\
\hline C & -5.82409 & 0.04994 & -1.26430 \\
\hline $\mathrm{H}$ & -5.80172 & 1.08695 & -1.63634 \\
\hline $\mathrm{H}$ & -6.42878 & -0.55113 & -1.96253 \\
\hline $\mathrm{H}$ & -6.34776 & 0.05693 & -0.29516 \\
\hline $\mathrm{Pd}$ & 1.70197 & -1.13459 & -0.01596 \\
\hline $\mathrm{Br}$ & -0.25801 & -2.48081 & 0.75214 \\
\hline C & 3.52108 & -0.33976 & -0.16086 \\
\hline C & 4.48919 & -1.15608 & -0.76466 \\
\hline C & 3.86412 & 0.46786 & 0.93150 \\
\hline C & 5.77455 & -1.21374 & -0.22778 \\
\hline $\mathrm{H}$ & 4.23691 & -1.75166 & -1.64624 \\
\hline C & 5.15260 & 0.39739 & 1.46097 \\
\hline $\mathrm{H}$ & 3.12676 & 1.14829 & 1.36435 \\
\hline
\end{tabular}




$\begin{array}{llll}\mathrm{C} & 6.10781 & -0.43991 & 0.88446 \\ \mathrm{H} & 6.52212 & -1.86464 & -0.68793 \\ \mathrm{H} & 5.41112 & 1.00998 & 2.32826 \\ \mathrm{H} & 7.11877 & -0.48121 & 1.29601 \\ \mathrm{57} & & & \end{array}$

\section{TS-D2}

\begin{tabular}{|c|c|c|c|}
\hline r & 0.57803 & 1.77296 & 1.71656 \\
\hline & 1.20940 & 1.95284 & 0.44854 \\
\hline & 1.97700 & 3.09300 & 0.18538 \\
\hline & 2.10252 & 4.06211 & 1.17145 \\
\hline & 1.46678 & 3.89710 & 2.41639 \\
\hline & 0.70544 & 2.77212 & 2.70489 \\
\hline & 0.08166 & 0.01907 & 0.46266 \\
\hline & 0.88360 & 0.79322 & -0.35826 \\
\hline & 2.48622 & 3.19495 & -0.77542 \\
\hline & 2.70429 & 4.95433 & 0.98780 \\
\hline & 1.58425 & 4.67015 & 3.18038 \\
\hline & 0.23493 & 2.64668 & 3.68395 \\
\hline & 0.81484 & 0.87124 & -1.45064 \\
\hline & -0.17774 & 0.63378 & 1.76925 \\
\hline & -1.34224 & 0.13949 & 0.00089 \\
\hline & -1.68593 & 1.75600 & 1.04885 \\
\hline & -0.84866 & -0.85444 & 2.68450 \\
\hline & -2.66854 & 0.53995 & -0.10599 \\
\hline Si & -2.82472 & 1.41144 & -1.61332 \\
\hline & -3.79510 & -0.63345 & 0.49046 \\
\hline & -2.01717 & 0.48149 & -3.03515 \\
\hline & -2.50424 & -0.49611 & -3.18013 \\
\hline & -2.09695 & 1.04088 & -3.9807 \\
\hline & -0.94812 & 0.29938 & -2.8450 \\
\hline
\end{tabular}




\begin{tabular}{|c|c|c|c|}
\hline C & -1.93753 & 3.06603 & -1.35945 \\
\hline $\mathrm{H}$ & -1.93565 & 3.65879 & -2.28799 \\
\hline $\mathrm{H}$ & -2.44697 & 3.68676 & -0.59964 \\
\hline $\mathrm{H}$ & -0.87944 & 2.95047 & -1.06564 \\
\hline C & -4.61299 & 1.78082 & -2.07212 \\
\hline $\mathrm{H}$ & -5.11937 & 2.36619 & -1.28906 \\
\hline $\mathrm{H}$ & -4.64940 & 2.36433 & -3.00625 \\
\hline $\mathrm{H}$ & -5.19280 & 0.85890 & -2.23662 \\
\hline C & -3.04536 & -1.47260 & 2.03389 \\
\hline $\mathrm{H}$ & -2.15591 & -2.07107 & 1.76462 \\
\hline $\mathrm{H}$ & -2.84415 & -0.71840 & 2.81963 \\
\hline $\mathrm{H}$ & -3.76645 & -2.17841 & 2.47356 \\
\hline C & -5.40209 & 0.15326 & 1.08210 \\
\hline $\mathrm{H}$ & -5.95593 & 0.60826 & 0.24739 \\
\hline $\mathrm{H}$ & -6.06064 & -0.59552 & 1.55141 \\
\hline $\mathrm{H}$ & -5.20844 & 0.94551 & 1.82350 \\
\hline C & -4.18637 & -2.00217 & -0.73792 \\
\hline $\mathrm{H}$ & -3.26803 & -2.53505 & -1.02885 \\
\hline $\mathrm{H}$ & -4.88901 & -2.73739 & -0.31449 \\
\hline $\mathrm{H}$ & -4.64387 & -1.59194 & -1.65223 \\
\hline $\mathrm{Pd}$ & 2.12783 & -0.82374 & -0.22095 \\
\hline $\mathrm{Br}$ & 4.26240 & -0.17969 & -1.16403 \\
\hline C & 0.14860 & -1.48868 & 0.45985 \\
\hline C & 0.61900 & -2.16475 & 1.62451 \\
\hline C & -0.32945 & -2.29592 & -0.60784 \\
\hline C & 0.59020 & -3.56142 & 1.71505 \\
\hline $\mathrm{H}$ & 1.08397 & -1.56958 & 2.42416 \\
\hline C & -0.34359 & -3.67874 & -0.50475 \\
\hline $\mathrm{H}$ & -0.70359 & -1.80486 & -1.50858 \\
\hline C & 0.09423 & -4.32220 & 0.66184 \\
\hline
\end{tabular}




$\begin{array}{llll}\mathrm{H} & 0.98005 & -4.05053 & 2.61135 \\ \mathrm{H} & -0.71036 & -4.27286 & -1.34534 \\ \mathrm{H} & 0.06240 & -5.41092 & 0.73439 \\ \mathrm{H} & & & \end{array}$

TS-D3

\begin{tabular}{|c|c|c|c|}
\hline$r$ & 0.06084 & 1.78697 & -1.37784 \\
\hline & 0.22552 & 1.76625 & 0.02992 \\
\hline & 0.56476 & 2.94160 & 0.69850 \\
\hline & 0.75048 & 4.11456 & -0.03626 \\
\hline & 0.61752 & 4.11983 & -1.42873 \\
\hline & 0.27256 & 2.95624 & -2.11654 \\
\hline & -0.49107 & -0.27019 & -0.77137 \\
\hline & 0.05897 & 0.36256 & 0.45379 \\
\hline & 0.68943 & 2.95184 & 1.78315 \\
\hline & 1.01340 & 5.03862 & 0.48302 \\
\hline & 0.77738 & 5.04705 & -1.98360 \\
\hline & 0.15012 & 2.95772 & -3.20318 \\
\hline & 1.26488 & -0.13353 & 0.29133 \\
\hline & -0.28406 & 0.52520 & -1.87921 \\
\hline & -0.42715 & -1.35667 & -0.93246 \\
\hline i & -2.12533 & 0.36689 & -2.49129 \\
\hline$i$ & 1.76897 & 0.21902 & -1.97579 \\
\hline $\mathrm{N}$ & 2.55721 & -0.49813 & -0.34408 \\
\hline $\mathrm{i}$ & 2.68649 & -2.11901 & -0.93951 \\
\hline & 3.71032 & 0.48083 & 0.52295 \\
\hline C & 4.41951 & -2.84507 & -1.06837 \\
\hline r. & 4.87428 & -3.01205 & -0.08112 \\
\hline & 4.36037 & -3.82010 & -1.57936 \\
\hline & 5.09753 & -2.20048 & -1.64770 \\
\hline & 2.03978 & -2.09145 & -2.74317 \\
\hline
\end{tabular}




\begin{tabular}{|c|c|c|c|}
\hline $\mathrm{H}$ & 2.05504 & -3.11091 & -3.15943 \\
\hline $\mathrm{H}$ & 1.00026 & -1.73561 & -2.85711 \\
\hline $\mathrm{H}$ & 2.69247 & -1.49116 & -3.40629 \\
\hline C & 1.63942 & -3.37320 & 0.00272 \\
\hline $\mathrm{H}$ & 0.56058 & -3.16417 & -0.05791 \\
\hline $\mathrm{H}$ & 1.80048 & -4.38639 & -0.39904 \\
\hline $\mathrm{H}$ & 1.92289 & -3.38556 & 1.06733 \\
\hline C & 3.94141 & 2.10736 & -0.41340 \\
\hline $\mathrm{H}$ & 4.44305 & 1.92138 & -1.37892 \\
\hline $\mathrm{H}$ & 2.98462 & 2.61621 & -0.61045 \\
\hline $\mathrm{H}$ & 4.57472 & 2.81199 & 0.14942 \\
\hline C & 3.08392 & 0.80768 & 2.27159 \\
\hline $\mathrm{H}$ & 3.04464 & -0.13933 & 2.83402 \\
\hline $\mathrm{H}$ & 3.75169 & 1.49592 & 2.81410 \\
\hline $\mathrm{H}$ & 2.07180 & 1.23510 & 2.28489 \\
\hline C & 5.43505 & -0.25854 & 0.72501 \\
\hline $\mathrm{H}$ & 5.93098 & -0.45806 & -0.23652 \\
\hline $\mathrm{H}$ & 6.05864 & 0.46350 & 1.27793 \\
\hline $\mathrm{H}$ & 5.42195 & -1.19427 & 1.30448 \\
\hline $\mathrm{Pd}$ & -2.35537 & -0.30777 & 0.20675 \\
\hline $\mathrm{Br}$ & -4.07315 & -0.54840 & -1.58584 \\
\hline C & -0.67330 & -0.01056 & 1.71990 \\
\hline C & -0.57459 & -1.31764 & 2.25505 \\
\hline C & -1.44932 & 0.93543 & 2.43331 \\
\hline C & -1.20744 & -1.65288 & 3.44660 \\
\hline $\mathrm{H}$ & 0.03351 & -2.05779 & 1.73408 \\
\hline C & -2.07619 & 0.58673 & 3.63207 \\
\hline $\mathrm{H}$ & -1.52929 & 1.95647 & 2.05586 \\
\hline C & -1.95328 & -0.70108 & 4.14628 \\
\hline $\mathrm{H}$ & -1.11062 & -2.66841 & 3.83756 \\
\hline
\end{tabular}




\begin{tabular}{|c|c|c|c|}
\hline $\mathrm{H}$ & -2.66526 & 1.33701 & 4.16467 \\
\hline $\mathrm{H}$ & -2.44070 & -0.96725 & 5.08668 \\
\hline \multicolumn{4}{|c|}{57} \\
\hline \multicolumn{4}{|c|}{ D2 } \\
\hline C & 0.37351 & 2.08124 & -0.70340 \\
\hline C & 1.77725 & 1.86057 & -0.82226 \\
\hline C & 2.70032 & 2.87556 & -0.55176 \\
\hline C & 2.23246 & 4.12346 & -0.15999 \\
\hline C & 0.84913 & 4.34734 & -0.03633 \\
\hline C & -0.08434 & 3.35202 & -0.29731 \\
\hline C & 0.54834 & -0.08017 & -1.34555 \\
\hline C & 1.96324 & 0.48762 & -1.25327 \\
\hline $\mathrm{H}$ & 3.76972 & 2.66240 & -0.62353 \\
\hline $\mathrm{H}$ & 2.93433 & 4.92900 & 0.06347 \\
\hline$H$ & 0.49641 & 5.33361 & 0.27752 \\
\hline $\mathrm{H}$ & -1.15469 & 3.55393 & -0.19593 \\
\hline $\mathrm{H}$ & 2.70029 & 0.25440 & -2.03004 \\
\hline . & -0.38618 & 0.98664 & -1.01104 \\
\hline $\mathrm{H}$ & 0.34706 & -0.53560 & -2.33161 \\
\hline Li & -1.51977 & 0.18974 & 0.48495 \\
\hline $\mathrm{Li}$ & -2.22836 & 0.97435 & -1.62769 \\
\hline$N$ & -3.32083 & 0.23142 & -0.22692 \\
\hline Si & -3.98292 & 1.07122 & 1.12235 \\
\hline Si & -4.01486 & -1.05860 & -1.12956 \\
\hline C & -4.75876 & 2.71983 & 0.64065 \\
\hline $\mathrm{H}$ & -5.60535 & 2.55532 & -0.04553 \\
\hline $\mathrm{H}$ & -5.13522 & 3.27482 & 1.51480 \\
\hline $\mathrm{H}$ & -4.02965 & 3.36424 & 0.12215 \\
\hline C & -2.50838 & 1.45465 & 2.27995 \\
\hline$H$ & -2.84390 & 2.05973 & 3.13635 \\
\hline
\end{tabular}




\begin{tabular}{|c|c|c|c|}
\hline $\mathrm{H}$ & -2.06683 & 0.53950 & 2.71889 \\
\hline $\mathrm{H}$ & -1.70381 & 2.04374 & 1.80021 \\
\hline C & -5.22331 & 0.08428 & 2.14126 \\
\hline $\mathrm{H}$ & -4.79220 & -0.87653 & 2.46462 \\
\hline $\mathrm{H}$ & -5.52812 & 0.63988 & 3.04264 \\
\hline $\mathrm{H}$ & -6.13467 & -0.13404 & 1.56300 \\
\hline C & -3.05779 & -1.09906 & -2.78402 \\
\hline $\mathrm{H}$ & -3.19980 & -0.18730 & -3.39659 \\
\hline $\mathrm{H}$ & -1.97479 & -1.26350 & -2.64234 \\
\hline $\mathrm{H}$ & -3.41362 & -1.92836 & -3.41515 \\
\hline C & -3.78641 & -2.74229 & -0.31503 \\
\hline $\mathrm{H}$ & -4.33460 & -2.78179 & 0.64003 \\
\hline $\mathrm{H}$ & -4.15644 & -3.56411 & -0.94902 \\
\hline $\mathrm{H}$ & -2.72222 & -2.92944 & -0.09783 \\
\hline C & -5.83621 & -0.82617 & -1.55743 \\
\hline $\mathrm{H}$ & -6.00543 & 0.14661 & -2.04649 \\
\hline $\mathrm{H}$ & -6.19387 & -1.61592 & -2.23758 \\
\hline $\mathrm{H}$ & -6.46487 & -0.85852 & -0.65374 \\
\hline $\mathrm{Pd}$ & 2.59506 & -0.75031 & 0.23725 \\
\hline $\mathrm{Br}$ & 4.96684 & -0.38169 & 0.03594 \\
\hline C & 0.50622 & -1.19709 & -0.27996 \\
\hline C & 0.50997 & -0.82955 & 1.10138 \\
\hline C & 0.30105 & -2.55661 & -0.61254 \\
\hline C & 0.31344 & -1.81586 & 2.09385 \\
\hline $\mathrm{H}$ & 0.52239 & 0.23001 & 1.39217 \\
\hline C & 0.09874 & -3.50573 & 0.37572 \\
\hline $\mathrm{H}$ & 0.28570 & -2.84397 & -1.66699 \\
\hline C & 0.10917 & -3.13809 & 1.73174 \\
\hline $\mathrm{H}$ & 0.31574 & -1.52261 & 3.14611 \\
\hline $\mathrm{H}$ & -0.07539 & -4.54752 & 0.09808 \\
\hline
\end{tabular}




\begin{tabular}{|c|c|c|c|}
\hline $\mathrm{H}$ & -0.05182 & -3.89564 & 2.50150 \\
\hline 57 & & & \\
\hline D3 & & & \\
\hline $\mathrm{C}$ & -0.60244 & 2.17694 & 0.69836 \\
\hline C & -1.98856 & 2.27076 & 0.92832 \\
\hline $\mathrm{C}$ & -2.68223 & 3.43341 & 0.63130 \\
\hline $\mathrm{C}$ & -1.97999 & 4.52454 & 0.10681 \\
\hline C & -0.60441 & 4.43278 & -0.11978 \\
\hline $\mathrm{C}$ & 0.10030 & 3.26266 & 0.16655 \\
\hline C & -1.13640 & 0.15472 & 1.43419 \\
\hline C & -2.44717 & 0.94623 & 1.46585 \\
\hline $\mathrm{H}$ & -3.76071 & 3.49709 & 0.79817 \\
\hline $\mathrm{H}$ & -2.50889 & 5.45021 & -0.12915 \\
\hline $\mathrm{H}$ & -0.06885 & 5.28929 & -0.53641 \\
\hline $\mathrm{H}$ & 1.17229 & 3.19480 & -0.03575 \\
\hline $\mathrm{H}$ & -2.85696 & 1.01954 & 2.48825 \\
\hline $\mathrm{N}$ & -0.08331 & 0.93718 & 1.07532 \\
\hline $\mathrm{H}$ & -0.95391 & -0.65798 & 2.15019 \\
\hline $\mathrm{Li}$ & 1.05230 & -0.35721 & -0.31484 \\
\hline $\mathrm{Li}$ & 1.82552 & 0.97851 & 1.54453 \\
\hline $\mathrm{N}$ & 2.87149 & 0.27440 & 0.13617 \\
\hline Si & 3.24175 & 0.84910 & -1.44484 \\
\hline Si & 3.92210 & -0.61751 & 1.16721 \\
\hline$C$ & 3.95154 & 2.59935 & -1.44719 \\
\hline $\mathrm{H}$ & 4.90534 & 2.61949 & -0.89543 \\
\hline $\mathrm{H}$ & 4.14456 & 2.96269 & -2.46953 \\
\hline $\mathrm{H}$ & 3.27237 & 3.31677 & -0.95880 \\
\hline C & 1.60268 & 0.87686 & -2.41766 \\
\hline $\mathrm{H}$ & 1.76234 & 1.29241 & -3.42504 \\
\hline $\mathrm{H}$ & 1.18616 & -0.13624 & -2.57414 \\
\hline
\end{tabular}




\begin{tabular}{|c|c|c|c|}
\hline $\mathrm{H}$ & 0.82825 & 1.50395 & -1.94151 \\
\hline C & 4.42590 & -0.26094 & -2.40667 \\
\hline $\mathrm{H}$ & 4.02189 & -1.28299 & -2.48217 \\
\hline $\mathrm{H}$ & 4.58733 & 0.11745 & -3.42896 \\
\hline $\mathrm{H}$ & 5.41079 & -0.32440 & -1.91700 \\
\hline C & 3.11191 & -0.50300 & 2.90318 \\
\hline $\mathrm{H}$ & 3.08297 & 0.52300 & 3.32242 \\
\hline $\mathrm{H}$ & 2.09064 & -0.92736 & 2.91994 \\
\hline $\mathrm{H}$ & 3.69224 & -1.08927 & 3.63202 \\
\hline C & 4.07028 & -2.44393 & 0.75018 \\
\hline $\mathrm{H}$ & 4.55239 & -2.57416 & -0.23164 \\
\hline $\mathrm{H}$ & 4.67071 & -2.98815 & 1.49692 \\
\hline $\mathrm{H}$ & 3.07247 & -2.90724 & 0.69462 \\
\hline C & 5.65204 & 0.12092 & 1.33004 \\
\hline $\mathrm{H}$ & 5.60388 & 1.18079 & 1.62765 \\
\hline $\mathrm{H}$ & 6.25598 & -0.41816 & 2.07776 \\
\hline $\mathrm{H}$ & 6.19188 & 0.07088 & 0.37084 \\
\hline $\mathrm{Pd}$ & -1.78584 & -1.09594 & -0.13895 \\
\hline $\mathrm{Br}$ & 0.15608 & -2.61212 & -0.39987 \\
\hline C & -3.42605 & 0.18571 & 0.56589 \\
\hline C & -4.41008 & -0.67492 & 1.11063 \\
\hline C & -3.39689 & 0.37900 & -0.84088 \\
\hline C & -5.34035 & -1.29555 & 0.29162 \\
\hline $\mathrm{H}$ & -4.44294 & -0.82510 & 2.19292 \\
\hline C & -4.33809 & -0.27934 & -1.65852 \\
\hline $\mathrm{H}$ & -2.75968 & 1.15761 & -1.26924 \\
\hline C & -5.30435 & -1.10071 & -1.09738 \\
\hline $\mathrm{H}$ & -6.10208 & -1.94264 & 0.73202 \\
\hline $\mathrm{H}$ & -4.31427 & -0.11167 & -2.73747 \\
\hline $\mathrm{H}$ & -6.04091 & -1.59323 & -1.73588 \\
\hline
\end{tabular}


57

\begin{tabular}{|c|c|c|c|}
\hline \multicolumn{4}{|c|}{3 prime } \\
\hline C & 0.18593 & 1.57590 & -1.58745 \\
\hline & 0.23892 & 2.05611 & -0.26342 \\
\hline & 0.52996 & 3.38643 & -0.00632 \\
\hline 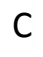 & 0.77518 & 4.24588 & -1.08331 \\
\hline & 0.72026 & 3.76905 & -2.39458 \\
\hline & 0.42354 & 2.43187 & -2.66515 \\
\hline C & -0.32916 & -0.20156 & -0.35306 \\
\hline & -0.05391 & 0.90569 & 0.65434 \\
\hline . & 0.57171 & 3.75824 & 1.02038 \\
\hline H & 1.01612 & 5.29439 & -0.89759 \\
\hline 1 & 0.91603 & 4.45224 & -3.22428 \\
\hline r & 0.38487 & 2.06316 & -3.69338 \\
\hline . & 0.81249 & 0.64258 & 1.28359 \\
\hline ] & -0.06391 & 0.19745 & -1.64253 \\
\hline 4 & -0.11257 & -1.24374 & -0.08701 \\
\hline i & -1.44307 & -0.75725 & -2.59232 \\
\hline Li & 1.92908 & -0.27971 & -1.67407 \\
\hline N & 2.90773 & -0.77249 & -0.21592 \\
\hline $\mathrm{Si}$ & 2.66063 & -2.32722 & 0.43776 \\
\hline & 4.03459 & 0.44887 & 0.17494 \\
\hline ᄃ & 4.23788 & -3.29656 & 0.82628 \\
\hline | & 4.79134 & -2.83875 & 1.66157 \\
\hline I & 4.00678 & -4.33658 & 1.10937 \\
\hline H & 4.91164 & -3.31890 & -0.04486 \\
\hline 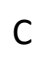 & 1.68558 & -3.38192 & -0.81316 \\
\hline 1 & 1.48658 & -4.39093 & -0.41718 \\
\hline H & 0.70358 & -2.95237 & -1.07916 \\
\hline & 2.26355 & -3.50385 & -1.74 \\
\hline
\end{tabular}




\begin{tabular}{|c|c|c|c|}
\hline C & 1.64645 & -2.32226 & 2.04808 \\
\hline $\mathrm{H}$ & 0.61546 & -1.96473 & 1.89237 \\
\hline $\mathrm{H}$ & 1.57761 & -3.33153 & 2.48607 \\
\hline $\mathrm{H}$ & 2.11949 & -1.66338 & 2.79506 \\
\hline C & 3.98664 & 1.75647 & -1.20758 \\
\hline $\mathrm{H}$ & 4.27683 & 1.32383 & -2.18172 \\
\hline $\mathrm{H}$ & 2.99256 & 2.22366 & -1.31370 \\
\hline $\mathrm{H}$ & 4.69562 & 2.57454 & -1.00111 \\
\hline C & 3.64488 & 1.36538 & 1.79111 \\
\hline $\mathrm{H}$ & 3.58665 & 0.66022 & 2.63650 \\
\hline $\mathrm{H}$ & 4.41723 & 2.11418 & 2.03213 \\
\hline $\mathrm{H}$ & 2.68004 & 1.89423 & 1.72613 \\
\hline C & 5.83178 & -0.12929 & 0.30155 \\
\hline $\mathrm{H}$ & 6.12745 & -0.68575 & -0.60227 \\
\hline $\mathrm{H}$ & 6.51784 & 0.72589 & 0.41749 \\
\hline $\mathrm{H}$ & 5.98051 & -0.79749 & 1.16408 \\
\hline $\mathrm{Pd}$ & -2.38387 & -0.29444 & 0.05105 \\
\hline $\mathrm{Br}$ & -3.13486 & -2.02463 & -1.59519 \\
\hline C & -1.32890 & 1.02105 & 1.49244 \\
\hline C & -1.43122 & 0.37879 & 2.75049 \\
\hline C & -2.41988 & 1.80600 & 1.03775 \\
\hline C & -2.56630 & 0.53409 & 3.53105 \\
\hline $\mathrm{H}$ & -0.59148 & -0.22305 & 3.10677 \\
\hline C & -3.57217 & 1.93673 & 1.83816 \\
\hline $\mathrm{H}$ & -2.30463 & 2.42960 & 0.14696 \\
\hline C & -3.64079 & 1.31229 & 3.07496 \\
\hline $\mathrm{H}$ & -2.62204 & 0.04487 & 4.50586 \\
\hline $\mathrm{H}$ & -4.39896 & 2.55722 & 1.48580 \\
\hline $\mathrm{H}$ & -4.53011 & 1.43092 & 3.69779 \\
\hline 5 & & & \\
\hline
\end{tabular}




\section{C3 prime}

\begin{tabular}{|c|c|c|c|}
\hline & -1.64731 & 2.26275 & -0.89860 \\
\hline & -0.78616 & 1.65662 & 0.05622 \\
\hline & -0.39883 & .35436 & 1.20972 \\
\hline & -0.87820 & 3.64890 & 1.39134 \\
\hline & -1.73972 & 4.23812 & 0.45137 \\
\hline & -2.13579 & 3.55769 & -0.69583 \\
\hline & -1.19617 & 0.29930 & -1.72654 \\
\hline & -0.49642 & 0.32008 & -0.46161 \\
\hline & 0.25162 & 1.88669 & 1.95294 \\
\hline & -0.58702 & 4.21148 & 2.28074 \\
\hline & -2.10167 & 5.25419 & 0.62398 \\
\hline & -2.79500 & 4.02779 & -1.42979 \\
\hline & 0.46223 & -0.18186 & -0.28776 \\
\hline & -1.90279 & 1.40841 & -1.96419 \\
\hline & -3.78468 & 0.98314 & -1.90906 \\
\hline & 1.44015 & 1.95187 & -0.73761 \\
\hline & 2.86180 & 0.84909 & -0.51669 \\
\hline & 3.35731 & -0.43654 & -1.53641 \\
\hline & 3.68469 & 1.33367 & 0.90444 \\
\hline & 4.83514 & 0.01892 & -2.62928 \\
\hline & 5.71666 & 0.26312 & -2.01589 \\
\hline & 5.11292 & -0.80421 & -3.30821 \\
\hline & 4.60450 & 0.90489 & -3.24325 \\
\hline & 1.95535 & -0.93246 & -2.72123 \\
\hline & 2.33773 & -1.60668 & -3.50476 \\
\hline$\Pi$ & 1.15830 & -1.47287 & -2.18561 \\
\hline $\mathrm{H}$ & 1.50790 & -0.06067 & -3.22888 \\
\hline & 3.81270 & -2.01280 & -0.5904 \\
\hline & 2.96434 & -2.34894 & 0.02 \\
\hline
\end{tabular}




\begin{tabular}{|c|c|c|c|}
\hline $\mathrm{H}$ & 4.08181 & -2.83451 & -1.27437 \\
\hline $\mathrm{H}$ & 4.67012 & -1.84537 & 0.08155 \\
\hline C & 3.27001 & 3.16274 & 1.24715 \\
\hline $\mathrm{H}$ & 3.69770 & 3.80648 & 0.46005 \\
\hline $\mathrm{H}$ & 2.18676 & 3.37241 & 1.30289 \\
\hline $\mathrm{H}$ & 3.69617 & 3.49298 & 2.20862 \\
\hline C & 3.14106 & 0.34884 & 2.43269 \\
\hline $\mathrm{H}$ & 3.35546 & -0.72208 & 2.28659 \\
\hline $\mathrm{H}$ & 3.65173 & 0.67983 & 3.35187 \\
\hline $\mathrm{H}$ & 2.05431 & 0.43834 & 2.59690 \\
\hline C & 5.57256 & 1.23901 & 0.83158 \\
\hline $\mathrm{H}$ & 5.96300 & 1.84678 & -0.00011 \\
\hline $\mathrm{H}$ & 6.02553 & 1.60766 & 1.76644 \\
\hline $\mathrm{H}$ & 5.91819 & 0.20408 & 0.67923 \\
\hline $\mathrm{Pd}$ & -2.07084 & -0.91941 & 0.23557 \\
\hline $\mathrm{Br}$ & -4.54098 & -0.12734 & 0.00859 \\
\hline C & -0.60997 & -2.14798 & 0.58308 \\
\hline C & -0.37120 & -3.20154 & -0.30232 \\
\hline C & 0.07226 & -2.07011 & 1.79930 \\
\hline C & 0.50452 & -4.22323 & 0.07163 \\
\hline $\mathrm{H}$ & -0.86944 & -3.24188 & -1.27477 \\
\hline C & 0.94417 & -3.09851 & 2.16225 \\
\hline $\mathrm{H}$ & -0.06799 & -1.21585 & 2.46679 \\
\hline C & 1.15894 & -4.17390 & 1.30158 \\
\hline $\mathrm{H}$ & 0.68399 & -5.05598 & -0.61283 \\
\hline $\mathrm{H}$ & 1.47364 & -3.04189 & 3.11645 \\
\hline $\mathrm{H}$ & 1.85673 & -4.96627 & 1.58081 \\
\hline $\mathrm{H}$ & -1.12631 & -0.49582 & -2.47196 \\
\hline & & & \\
\hline
\end{tabular}

TS-E2 


\begin{tabular}{|c|c|c|c|}
\hline C & 1.79433 & 2.27436 & 0.67771 \\
\hline C & 1.72314 & 2.51396 & -0.73690 \\
\hline C & 2.44762 & 3.58332 & -1.31413 \\
\hline C & 3.21432 & 4.38296 & -0.49444 \\
\hline C & 3.27405 & 4.14606 & 0.90368 \\
\hline C & 2.57469 & 3.11539 & 1.50060 \\
\hline C & 0.43169 & 0.72370 & -0.16011 \\
\hline C & 0.85206 & 1.53251 & -1.24900 \\
\hline $\mathrm{H}$ & 2.39717 & 3.76450 & -2.39031 \\
\hline $\mathrm{H}$ & 3.78585 & 5.21190 & -0.91747 \\
\hline $\mathrm{H}$ & 3.89754 & 4.79742 & 1.52167 \\
\hline $\mathrm{H}$ & 2.63607 & 2.93868 & 2.57736 \\
\hline $\mathrm{H}$ & 0.51497 & 1.41027 & -2.27771 \\
\hline$N$ & 1.02600 & 1.21039 & 1.03705 \\
\hline $\mathrm{H}$ & -0.79592 & 0.45770 & 0.12558 \\
\hline $\mathrm{Li}$ & 1.38118 & -0.52932 & 1.73397 \\
\hline $\mathrm{Li}$ & -0.75667 & 1.72156 & 1.71585 \\
\hline$N$ & -2.03758 & 0.54136 & 0.98802 \\
\hline $\mathrm{Si}$ & -2.29002 & -0.91994 & 1.87880 \\
\hline Si & -3.30154 & 1.36301 & 0.10194 \\
\hline C & -0.62446 & -1.41999 & 2.69210 \\
\hline $\mathrm{H}$ & -0.11259 & -0.57703 & 3.19413 \\
\hline $\mathrm{H}$ & -0.78663 & -2.18507 & 3.46662 \\
\hline $\mathrm{H}$ & 0.01162 & -1.90227 & 1.92653 \\
\hline C & -2.79920 & -2.41798 & 0.86884 \\
\hline $\mathrm{H}$ & -2.67508 & -3.34679 & 1.44930 \\
\hline $\mathrm{H}$ & -3.85292 & -2.35258 & 0.56009 \\
\hline $\mathrm{H}$ & -2.19576 & -2.48301 & -0.05098 \\
\hline C & -3.52008 & -0.68246 & 3.29090 \\
\hline $\mathrm{H}$ & -4.51821 & -0.43923 & 2.89243 \\
\hline
\end{tabular}




\begin{tabular}{|c|c|c|c|}
\hline $\mathrm{H}$ & -3.61918 & -1.59761 & 3.89714 \\
\hline $\mathrm{H}$ & -3.22224 & 0.14180 & 3.95906 \\
\hline C & -2.51783 & 2.38047 & -1.27494 \\
\hline $\mathrm{H}$ & -1.73042 & 3.06432 & -0.91622 \\
\hline $\mathrm{H}$ & -2.06986 & 1.70828 & -2.02377 \\
\hline $\mathrm{H}$ & -3.28377 & 2.99274 & -1.77733 \\
\hline C & -4.57463 & 0.21058 & -0.66248 \\
\hline $\mathrm{H}$ & -5.14592 & -0.34367 & 0.09905 \\
\hline $\mathrm{H}$ & -5.29521 & 0.78535 & -1.26637 \\
\hline $\mathrm{H}$ & -4.07287 & -0.51473 & -1.32210 \\
\hline c & -4.20145 & 2.55983 & 1.26398 \\
\hline $\mathrm{H}$ & -3.51232 & 3.31436 & 1.68192 \\
\hline r & -4.99952 & 3.10695 & 0.73582 \\
\hline $\mathrm{H}$ & -4.66220 & 2.02642 & 2.11061 \\
\hline $\mathrm{Pd}$ & 0.68360 & -1.18092 & -0.91816 \\
\hline $\mathrm{Br}$ & -1.18548 & -1.23698 & -2.52826 \\
\hline C & 2.34810 & -1.54851 & 0.09979 \\
\hline c & 3.46292 & -0.69198 & 0.15970 \\
\hline c & 2.47442 & -2.83888 & 0.65414 \\
\hline c & 4.66328 & -1.11829 & 0.73360 \\
\hline r & 3.39746 & 0.31775 & -0.25644 \\
\hline C & 3.67646 & -3.26180 & 1.22913 \\
\hline r & 1.62741 & -3.53458 & 0.62812 \\
\hline c & 4.77272 & -2.40113 & 1.27083 \\
\hline $\mathrm{H}$ & 5.52081 & -0.44049 & 0.75535 \\
\hline r & 3.75245 & -4.26914 & 1.64700 \\
\hline$\vdash$ & 5.71185 & -2.73030 & 1.72172 \\
\hline \multicolumn{4}{|l|}{57} \\
\hline \multicolumn{4}{|c|}{ TS-E3 } \\
\hline C & 1.45813 & -2.64099 & -0.09968 \\
\hline
\end{tabular}




\begin{tabular}{|c|c|c|c|}
\hline C & 0.84168 & -1.60115 & 0.65260 \\
\hline C & 0.59377 & -1.79878 & 2.02122 \\
\hline C & 0.97263 & -2.99970 & 2.60422 \\
\hline C & 1.58587 & -4.01953 & 1.85003 \\
\hline C & 1.83114 & -3.85618 & 0.49428 \\
\hline C & 1.06795 & -1.03392 & -1.51274 \\
\hline C & 0.54363 & -0.51164 & -0.28118 \\
\hline $\mathrm{H}$ & 0.11171 & -1.01698 & 2.61002 \\
\hline $\mathrm{H}$ & 0.78907 & -3.16222 & 3.66884 \\
\hline $\mathrm{H}$ & 1.86468 & -4.95485 & 2.34076 \\
\hline $\mathrm{H}$ & 2.28981 & -4.64825 & -0.10264 \\
\hline $\mathrm{H}$ & -0.76193 & -0.25422 & -0.41755 \\
\hline$N$ & 1.59804 & -2.26533 & -1.43492 \\
\hline $\mathrm{Li}$ & 3.42009 & -1.55198 & -1.59383 \\
\hline $\mathrm{Li}$ & -0.96311 & -2.08741 & -0.85898 \\
\hline$N$ & -2.11511 & -0.54605 & -0.80621 \\
\hline $\mathrm{Si}$ & -2.62736 & 0.51109 & -2.08854 \\
\hline Si & -3.15353 & -1.15689 & 0.44608 \\
\hline C & -3.59929 & -0.42581 & -3.41117 \\
\hline $\mathrm{H}$ & -4.53721 & -0.83813 & -3.00809 \\
\hline $\mathrm{H}$ & -3.85956 & 0.23524 & -4.25418 \\
\hline $\mathrm{H}$ & -3.00765 & -1.26440 & -3.81397 \\
\hline C & -1.11311 & 1.24420 & -2.95075 \\
\hline $\mathrm{H}$ & -1.41655 & 2.08394 & -3.59599 \\
\hline $\mathrm{H}$ & -0.35496 & 1.61703 & -2.24341 \\
\hline $\mathrm{H}$ & -0.62913 & 0.49792 & -3.60098 \\
\hline C & -3.72477 & 1.92813 & -1.49050 \\
\hline $\mathrm{H}$ & -3.27003 & 2.46176 & -0.64341 \\
\hline $\mathrm{H}$ & -3.90177 & 2.65430 & -2.30034 \\
\hline $\mathrm{H}$ & -4.70779 & 1.55877 & -1.15961 \\
\hline
\end{tabular}




\begin{tabular}{|c|c|c|c|}
\hline C & -2.57006 & -2.94817 & 0.78084 \\
\hline $\mathrm{H}$ & -2.70741 & -3.59188 & -0.10941 \\
\hline $\mathrm{H}$ & -1.53064 & -3.04288 & 1.14310 \\
\hline $\mathrm{H}$ & -3.19140 & -3.40010 & 1.56999 \\
\hline$C$ & -3.04184 & -0.16144 & 2.03269 \\
\hline $\mathrm{H}$ & -3.49308 & 0.83162 & 1.88176 \\
\hline $\mathrm{H}$ & -3.56519 & -0.65299 & 2.86823 \\
\hline $\mathrm{H}$ & -1.99501 & 0.01054 & 2.32471 \\
\hline$C$ & -4.96511 & -1.32700 & -0.04885 \\
\hline $\mathrm{H}$ & -5.08247 & -1.96940 & -0.93527 \\
\hline $\mathrm{H}$ & -5.53652 & -1.78201 & 0.77666 \\
\hline $\mathrm{H}$ & -5.42557 & -0.35297 & -0.27227 \\
\hline $\mathrm{Pd}$ & 1.68436 & 1.15542 & 0.07825 \\
\hline $\mathrm{Br}$ & 3.98220 & 0.66746 & -1.10636 \\
\hline$C$ & 0.25474 & 1.81678 & 1.20346 \\
\hline$C$ & -0.78565 & 2.60678 & 0.70539 \\
\hline C & 0.42058 & 1.68743 & 2.58917 \\
\hline C & -1.61892 & 3.29990 & 1.58510 \\
\hline $\mathrm{H}$ & -0.94334 & 2.69542 & -0.37087 \\
\hline C & -0.42121 & 2.37876 & 3.46364 \\
\hline $\mathrm{H}$ & 1.21905 & 1.05871 & 2.99474 \\
\hline C & -1.44272 & 3.18488 & 2.96343 \\
\hline $\mathrm{H}$ & -2.41923 & 3.92979 & 1.18678 \\
\hline $\mathrm{H}$ & -0.27765 & 2.28105 & 4.54307 \\
\hline $\mathrm{H}$ & -2.10532 & 3.71925 & 3.64822 \\
\hline $\mathrm{H}$ & 1.04375 & -0.51105 & -2.47221 \\
\hline \multicolumn{4}{|l|}{57} \\
\hline \multicolumn{4}{|l|}{ E2 } \\
\hline C & -0.19627 & 2.40055 & 0.39914 \\
\hline C & -0.37547 & 2.64389 & -1.00174 \\
\hline
\end{tabular}




\begin{tabular}{|c|c|c|c|}
\hline C & -1.29399 & 3.63009 & -1.41882 \\
\hline C & -1.99395 & 4.34306 & -0.45992 \\
\hline C & -1.80250 & 4.10239 & 0.91904 \\
\hline C & -0.91163 & 3.13667 & 1.35922 \\
\hline C & 1.11405 & 0.99267 & -0.65780 \\
\hline C & 0.48376 & 1.71457 & -1.67429 \\
\hline $\mathrm{H}$ & -1.44326 & 3.83337 & -2.48239 \\
\hline $\mathrm{H}$ & -2.70667 & 5.11043 & -0.77175 \\
\hline $\mathrm{H}$ & -2.36928 & 4.68740 & 1.64736 \\
\hline $\mathrm{H}$ & -0.75788 & 2.94964 & 2.42515 \\
\hline $\mathrm{H}$ & 0.60985 & 1.57848 & -2.74573 \\
\hline$N$ & 0.70197 & 1.37969 & 0.58767 \\
\hline $\mathrm{H}$ & -1.91353 & -1.60690 & -0.30574 \\
\hline $\mathrm{Li}$ & 1.82915 & 0.23362 & 1.53913 \\
\hline $\mathrm{Li}$ & -0.87149 & 0.27706 & -0.53241 \\
\hline$N$ & -2.29196 & -0.89581 & 0.33864 \\
\hline Si & -1.99521 & -1.47436 & 1.98795 \\
\hline $\mathrm{Si}$ & -3.81876 & -0.26951 & -0.34222 \\
\hline C & -0.16160 & -1.26113 & 2.40002 \\
\hline $\mathrm{H}$ & 0.01113 & -0.18901 & 2.58985 \\
\hline $\mathrm{H}$ & 0.10931 & -1.83831 & 3.29749 \\
\hline $\mathrm{H}$ & 0.44993 & -1.61369 & 1.54938 \\
\hline C & -2.42228 & -3.29791 & 2.08179 \\
\hline $\mathrm{H}$ & -2.22627 & -3.70845 & 3.08496 \\
\hline $\mathrm{H}$ & -3.48414 & -3.46758 & 1.84486 \\
\hline $\mathrm{H}$ & -1.82021 & -3.87114 & 1.35821 \\
\hline C & -2.99457 & -0.47108 & 3.21250 \\
\hline $\mathrm{H}$ & -4.07737 & -0.62413 & 3.08770 \\
\hline $\mathrm{H}$ & -2.73209 & -0.77342 & 4.23883 \\
\hline $\mathrm{H}$ & -2.78410 & 0.60459 & 3.10890 \\
\hline
\end{tabular}




\begin{tabular}{|c|c|c|c|}
\hline C & -3.43151 & 0.09999 & -2.14182 \\
\hline $\mathrm{H}$ & -2.72813 & 0.94524 & -2.23653 \\
\hline $\mathrm{H}$ & -2.99001 & -0.76721 & -2.65741 \\
\hline $\mathrm{H}$ & -4.34955 & 0.38683 & -2.67868 \\
\hline C & -5.17825 & -1.55799 & -0.20451 \\
\hline $\mathrm{H}$ & -5.40039 & -1.78976 & 0.84951 \\
\hline $\mathrm{H}$ & -6.11037 & -1.20101 & -0.67071 \\
\hline $\mathrm{H}$ & -4.88766 & -2.49580 & -0.70387 \\
\hline C & -4.30865 & 1.31314 & 0.52467 \\
\hline $\mathrm{H}$ & -3.46915 & 2.02562 & 0.56066 \\
\hline $\mathrm{H}$ & -5.12420 & 1.79059 & -0.04215 \\
\hline $\mathrm{H}$ & -4.66839 & 1.14805 & 1.55004 \\
\hline $\mathrm{Pd}$ & 2.04103 & -0.70605 & -0.87522 \\
\hline $\mathrm{Br}$ & 0.08473 & -1.91660 & -1.90733 \\
\hline C & 3.60923 & -0.04342 & 0.16844 \\
\hline C & 4.08041 & 1.27981 & 0.27782 \\
\hline C & 4.34475 & -1.05151 & 0.82842 \\
\hline C & 5.24212 & 1.57386 & 0.99525 \\
\hline $\mathrm{H}$ & 3.53357 & 2.08804 & -0.21373 \\
\hline C & 5.50575 & -0.75647 & 1.54748 \\
\hline $\mathrm{H}$ & 4.01551 & -2.09658 & 0.77421 \\
\hline C & 5.95691 & 0.55990 & 1.63237 \\
\hline $\mathrm{H}$ & 5.59361 & 2.60765 & 1.05284 \\
\hline $\mathrm{H}$ & 6.05761 & -1.55982 & 2.04296 \\
\hline $\mathrm{H}$ & 6.86399 & 0.79489 & 2.19447 \\
\hline \multicolumn{4}{|l|}{57} \\
\hline \multicolumn{4}{|l|}{ E3 } \\
\hline C & -0.28004 & 2.30221 & -1.23389 \\
\hline C & -1.04310 & 1.64645 & -0.19606 \\
\hline C & -1.30415 & 2.32067 & 1.02546 \\
\hline
\end{tabular}




\begin{tabular}{|c|c|c|c|}
\hline C & -0.82527 & 3.60683 & 1.18659 \\
\hline C & -0.08234 & 4.24945 & 0.16366 \\
\hline C & 0.19717 & 3.61677 & -1.03248 \\
\hline C & -0.82845 & 0.36445 & -2.03056 \\
\hline C & -1.40109 & 0.36027 & -0.73794 \\
\hline $\mathrm{H}$ & -1.87661 & 1.82347 & 1.81419 \\
\hline $\mathrm{H}$ & -1.01947 & 4.14205 & 2.11905 \\
\hline $\mathrm{H}$ & 0.27388 & 5.26902 & 0.32955 \\
\hline $\mathrm{H}$ & 0.76304 & 4.11352 & -1.82402 \\
\hline $\mathrm{H}$ & 2.21139 & -1.01721 & 0.50032 \\
\hline$N$ & -0.12935 & 1.49407 & -2.32282 \\
\hline $\mathrm{Li}$ & -3.20593 & 1.44680 & -0.96000 \\
\hline $\mathrm{Li}$ & 0.81750 & 0.44995 & -0.66074 \\
\hline$N$ & 2.58596 & -0.16305 & 0.06503 \\
\hline Si & 3.58937 & -0.65690 & -1.33611 \\
\hline Si & 3.16411 & 0.90832 & 1.37891 \\
\hline$C$ & 4.70264 & 0.76092 & -1.84963 \\
\hline $\mathrm{H}$ & 5.43282 & 1.03932 & -1.07518 \\
\hline $\mathrm{H}$ & 5.26834 & 0.45377 & -2.74389 \\
\hline $\mathrm{H}$ & 4.11765 & 1.65372 & -2.11787 \\
\hline$C$ & 2.44768 & -1.06425 & -2.76605 \\
\hline $\mathrm{H}$ & 3.03809 & -1.41292 & -3.62855 \\
\hline $\mathrm{H}$ & 1.73451 & -1.85991 & -2.50345 \\
\hline $\mathrm{H}$ & 1.87564 & -0.17773 & -3.08753 \\
\hline$C$ & 4.61531 & -2.15851 & -0.87208 \\
\hline $\mathrm{H}$ & 3.96841 & -2.99423 & -0.56449 \\
\hline $\mathrm{H}$ & 5.21823 & -2.49643 & -1.72992 \\
\hline $\mathrm{H}$ & 5.30142 & -1.93558 & -0.04075 \\
\hline$C$ & 3.36240 & 2.65361 & 0.73361 \\
\hline $\mathrm{H}$ & 4.17681 & 2.75741 & 0.00403 \\
\hline
\end{tabular}




\begin{tabular}{|c|c|c|c|}
\hline $\mathrm{H}$ & 2.42974 & 3.01236 & 0.27102 \\
\hline $\mathrm{H}$ & 3.58482 & 3.32052 & 1.58219 \\
\hline C & 1.85864 & 0.90681 & 2.72262 \\
\hline $\mathrm{H}$ & 1.72819 & -0.08875 & 3.17068 \\
\hline $\mathrm{H}$ & 2.15706 & 1.60283 & 3.52286 \\
\hline $\mathrm{H}$ & 0.88399 & 1.24426 & 2.33574 \\
\hline$C$ & 4.78874 & 0.26908 & 2.07650 \\
\hline $\mathrm{H}$ & 5.59262 & 0.28407 & 1.32419 \\
\hline $\mathrm{H}$ & 5.11638 & 0.88911 & 2.92617 \\
\hline $\mathrm{H}$ & 4.68151 & -0.76633 & 2.43715 \\
\hline $\mathrm{Pd}$ & -2.50461 & -1.00342 & 0.15054 \\
\hline $\mathrm{Br}$ & -4.84565 & 0.03997 & -0.05126 \\
\hline C & -0.78064 & -1.90005 & 0.45011 \\
\hline C & -0.17545 & -2.71643 & -0.52190 \\
\hline$c$ & -0.19233 & -1.85627 & 1.72640 \\
\hline 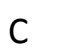 & 0.94249 & -3.49593 & -0.21712 \\
\hline $\mathrm{H}$ & -0.59287 & -2.75735 & -1.53164 \\
\hline C & 0.92560 & -2.63755 & 2.03369 \\
\hline$\pi$ & -0.61630 & -1.20961 & 2.49932 \\
\hline C & 1.49779 & -3.46083 & 1.06323 \\
\hline $\mathrm{H}$ & 1.38276 & -4.13746 & -0.98574 \\
\hline $\mathrm{H}$ & 1.35230 & -2.60352 & 3.04022 \\
\hline $\mathrm{H}$ & 2.37004 & -4.07328 & 1.30459 \\
\hline $\mathrm{H}$ & -0.87787 & -0.45012 & -2.75629 \\
\hline
\end{tabular}

\section{Post-TSD2}

$\begin{array}{llll}\text { C } & 0.39435 & 1.88082 & 0.32025 \\ \text { C } & 0.45783 & 1.78706 & -1.10891 \\ \text { C } & 0.17547 & 2.91436 & -1.89707 \\ \text { C } & -0.12839 & 4.11692 & -1.27242\end{array}$




\begin{tabular}{|c|c|c|c|}
\hline C & -0.15542 & 4.21473 & 0.13156 \\
\hline C & 0.10089 & 3.11139 & 0.93516 \\
\hline C & 0.86919 & -0.25543 & -0.11717 \\
\hline C & 0.79786 & 0.40823 & -1.41328 \\
\hline $\mathrm{H}$ & 0.21590 & 2.84794 & -2.98708 \\
\hline $\mathrm{H}$ & -0.33925 & 5.00246 & -1.87617 \\
\hline $\mathrm{H}$ & -0.38733 & 5.17566 & 0.59714 \\
\hline $\mathrm{H}$ & 0.06890 & 3.18584 & 2.02500 \\
\hline $\mathrm{H}$ & 0.59497 & -0.07795 & -2.37125 \\
\hline$N$ & 0.56965 & 0.65212 & 0.92701 \\
\hline $\mathrm{H}$ & -2.51521 & -1.29075 & 0.76443 \\
\hline $\mathrm{Li}$ & -1.17148 & 0.49304 & -0.14491 \\
\hline $\mathrm{Li}$ & 2.42840 & 0.46947 & 1.64175 \\
\hline$N$ & -2.88779 & -0.44526 & 0.32167 \\
\hline $\mathrm{Si}$ & -3.56271 & -0.85314 & -1.28279 \\
\hline $\mathrm{Si}$ & -3.58726 & 0.65310 & 1.54629 \\
\hline C & -2.92678 & -2.56426 & -1.68687 \\
\hline $\mathrm{H}$ & -3.34382 & -3.31646 & -0.99865 \\
\hline $\mathrm{H}$ & -3.20564 & -2.85533 & -2.71166 \\
\hline $\mathrm{H}$ & -1.82896 & -2.61106 & -1.60546 \\
\hline C & -2.90292 & 0.41647 & -2.50287 \\
\hline $\mathrm{H}$ & -3.33079 & 0.23869 & -3.50232 \\
\hline $\mathrm{H}$ & -3.16324 & 1.44646 & -2.21174 \\
\hline $\mathrm{H}$ & -1.80693 & 0.36357 & -2.61097 \\
\hline C & -5.43723 & -0.80156 & -1.30265 \\
\hline $\mathrm{H}$ & -5.82334 & 0.20505 & -1.07875 \\
\hline $\mathrm{H}$ & -5.80539 & -1.08161 & -2.30267 \\
\hline $\mathrm{H}$ & -5.86871 & -1.50538 & -0.57464 \\
\hline C & -2.37619 & 0.68282 & 2.97477 \\
\hline $\mathrm{H}$ & -2.35857 & -0.29113 & 3.49055 \\
\hline
\end{tabular}




\begin{tabular}{|c|c|c|c|}
\hline $\mathrm{H}$ & -1.35223 & 0.90111 & 2.63073 \\
\hline $\mathrm{H}$ & -2.66899 & 1.44442 & 3.71452 \\
\hline C & -3.72683 & 2.33342 & 0.72406 \\
\hline $\mathrm{H}$ & -4.40424 & 2.30832 & -0.14414 \\
\hline $\mathrm{H}$ & -4.13388 & 3.06829 & 1.43642 \\
\hline $\mathrm{H}$ & -2.74846 & 2.71767 & 0.38876 \\
\hline C & -5.26202 & 0.07175 & 2.16112 \\
\hline $\mathrm{H}$ & -5.20659 & -0.96489 & 2.52978 \\
\hline $\mathrm{H}$ & -5.59340 & 0.70538 & 2.99961 \\
\hline $\mathrm{H}$ & -6.03500 & 0.11510 & 1.38045 \\
\hline $\mathrm{Pd}$ & 2.82584 & 0.05009 & -0.79874 \\
\hline $\mathrm{Br}$ & 4.66582 & -0.04803 & 0.93393 \\
\hline C & 0.59816 & -1.69806 & 0.12542 \\
\hline C & 0.01406 & -2.11651 & 1.33131 \\
\hline C & 0.86826 & -2.66626 & -0.85529 \\
\hline C & -0.29905 & -3.45961 & 1.54493 \\
\hline $\mathrm{H}$ & -0.19266 & -1.36701 & 2.09833 \\
\hline C & 0.54689 & -4.00196 & -0.64331 \\
\hline $\mathrm{H}$ & 1.35917 & -2.36042 & -1.78239 \\
\hline C & -0.04145 & -4.40739 & 0.55749 \\
\hline $\mathrm{H}$ & -0.74544 & -3.76594 & 2.49466 \\
\hline $\mathrm{H}$ & 0.76763 & -4.73881 & -1.41924 \\
\hline $\mathrm{H}$ & -0.28523 & -5.45908 & 0.72382 \\
\hline 5 & & & \\
\hline
\end{tabular}

\section{Post-TSD3}

$\begin{array}{llll}\text { C } & -0.71205 & 1.74761 & 0.91883 \\ \text { C } & -0.52803 & 0.44108 & 1.48396 \\ \text { C } & -0.21290 & 0.32229 & 2.84941 \\ \text { C } & -0.11980 & 1.46591 & 3.63116 \\ \text { C } & -0.32838 & 2.74192 & 3.07635\end{array}$




\begin{tabular}{|c|c|c|c|}
\hline C & -0.61676 & 2.89443 & 1.72798 \\
\hline C & -0.98095 & 0.33079 & -0.76159 \\
\hline C & -0.68362 & -0.51260 & 0.38367 \\
\hline $\mathrm{H}$ & -0.05756 & -0.65747 & 3.30307 \\
\hline $\mathrm{H}$ & 0.11333 & 1.37330 & 4.69419 \\
\hline $\mathrm{H}$ & -0.25578 & 3.62488 & 3.71562 \\
\hline $\mathrm{H}$ & -0.76535 & 3.88343 & 1.28748 \\
\hline $\mathrm{H}$ & 2.38311 & -0.80744 & -0.71079 \\
\hline $\mathrm{N}$ & -0.90125 & 1.70222 & -0.45147 \\
\hline $\mathrm{H}$ & -0.83769 & 0.04283 & -1.80942 \\
\hline $\mathrm{Li}$ & -2.84794 & 1.89972 & -0.82976 \\
\hline $\mathrm{Li}$ & 1.00042 & 1.03967 & -0.14052 \\
\hline $\mathrm{N}$ & 2.74124 & 0.14720 & -0.58610 \\
\hline Si & 3.25237 & 0.82786 & -2.15343 \\
\hline Si & 3.70049 & 0.19988 & 0.92990 \\
\hline$C$ & 4.59963 & -0.20865 & -2.94369 \\
\hline $\mathrm{H}$ & 4.26963 & -1.25346 & -3.05934 \\
\hline $\mathrm{H}$ & 4.84686 & 0.17602 & -3.94595 \\
\hline $\mathrm{H}$ & 5.52113 & -0.20860 & -2.34323 \\
\hline$C$ & 3.82823 & 2.58576 & -1.84259 \\
\hline $\mathrm{H}$ & 4.10984 & 3.05790 & -2.79699 \\
\hline $\mathrm{H}$ & 3.02778 & 3.19780 & -1.39681 \\
\hline $\mathrm{H}$ & 4.70428 & 2.63371 & -1.17849 \\
\hline$C$ & 1.74512 & 0.85390 & -3.27242 \\
\hline $\mathrm{H}$ & 0.93520 & 1.47950 & -2.86247 \\
\hline $\mathrm{H}$ & 2.01489 & 1.26735 & -4.25730 \\
\hline $\mathrm{H}$ & 1.34387 & -0.15808 & -3.44175 \\
\hline C & 3.27097 & 1.80600 & 1.79931 \\
\hline $\mathrm{H}$ & 3.51621 & 2.68409 & 1.18182 \\
\hline $\mathrm{H}$ & 2.20392 & 1.85967 & 2.07573 \\
\hline
\end{tabular}




\begin{tabular}{llll} 
H & 3.84263 & 1.89336 & 2.73698 \\
$\mathrm{C}$ & 3.17723 & -1.28534 & 1.93436 \\
$\mathrm{H}$ & 3.35376 & -2.22559 & 1.38794 \\
$\mathrm{H}$ & 3.74119 & -1.32908 & 2.87928 \\
$\mathrm{H}$ & 2.10615 & -1.24109 & 2.17894 \\
$\mathrm{C}$ & 5.54642 & 0.12569 & 0.59017 \\
$\mathrm{H}$ & 5.90190 & 0.96598 & -0.02530 \\
$\mathrm{H}$ & 6.09784 & 0.15907 & 1.54360 \\
$\mathrm{H}$ & 5.81784 & -0.81214 & 0.08103 \\
$\mathrm{Pd}$ & -2.78168 & -0.53049 & -0.16626 \\
$\mathrm{Br}$ & -4.87819 & 0.62399 & -0.96859 \\
$\mathrm{C}$ & -0.15034 & -1.88440 & 0.33319 \\
$\mathrm{C}$ & 0.48186 & -2.37846 & -0.82490 \\
$\mathrm{C}$ & -0.21577 & -2.73852 & 1.44862 \\
$\mathrm{C}$ & 1.04739 & -3.65038 & -0.85515 \\
$\mathrm{H}$ & 0.51568 & -1.75556 & -1.72242 \\
$\mathrm{C}$ & 0.35732 & -4.00620 & 1.41923 \\
$\mathrm{H}$ & -0.75204 & -2.41078 & 2.34029 \\
$\mathrm{C}$ & 1.00277 & -4.46958 & 0.27276 \\
$\mathrm{H}$ & 1.52714 & -4.00511 & -1.77121 \\
$\mathrm{H}$ & 0.29009 & -4.64575 & 2.30291 \\
$\mathrm{H}$ & 1.45051 & -5.46545 & 0.25251 \\
& & & \\
\hline
\end{tabular}




\section{References}

(1) Newkome, G. R.; Gupta, V. K.; Taylor, M. C. R.; Fronczek, F. R. Carbon-Palladium(II) Complexes of Dialkyl Malonates: Synthesis, $1 \mathrm{H}$ and 13C NMR Spectroscopy, and Single-Crystal Structure Analyses. Organometallics 1984, 3, 1549-1554.

(2) Cao, Q.; Hughes, N. L.; Muldoon, M. J. Synthesis of 2-Alkynoates by Palladium(II)-Catalyzed Oxidative Carbonylation of Terminal Alkynes and Alcohols. Chem. Eur. J. 2016, 22, 11982-11985.

(3) Ibaceta-Lizana, J. S. L.; Jackson, A. H.; Prasitpan, N.; Shannon, P. V. R. Electrophilic Substitution in Indoles. Part 13. The Synthesis and Rearrangement of 2-Deuteriospiro[Cyclopentane-3'-Indolenine]. J. Chem. Soc., Perkin Trans. 2 1987, 1221-1226.

(4) Li, G.; Nakamura, H. Synthesis of 2-Indolyltetrahydroquinolines by Zinc(II)-Catalyzed Intramolecular Hydroarylation-Redox Cross-Dehydrogenative Coupling of N-Propargylanilines with Indoles. Angew. Chem. Int. Ed. 2016, 55, 6758-6761.

(5) Ma, D.; Cai, Q. L-Proline Promoted Ullmann-Type Coupling Reactions of Aryl lodides with Indoles, Pyrroles, Imidazoles or Pyrazoles. Synlett 2004, No. 1, 128-130.

(6) Molander, G. A.; Biolatto, B. Palladium-Catalyzed Suzuki-Miyaura Cross-Coupling Reactions of Potassium Aryl- and Heteroaryltrifluoroborates. J. Org. Chem. 2003, 68, 4302-4314.

(7) Chen, J.; Wu, J. Transition-Metal-Free C3 Arylation of Indoles with Aryl Halides. Angew. Chem. Int. Ed. 2017, 56, 3951-3955.

(8) Frisch, M. J.; Trucks, G. W.; Schlegel, H. E.; Scuseria, G. E.; Robb, M. A.; Cheeseman, J. R.; Scalmani, G.; Barone, V.; Petersson, G. A.; O., F.; Foresman, J. B.; Fox, J. D. Gaussian 16. Gaussian, Inc., Wallingford CT,. 2016.

(9) Adamo, C.; Barone, V. Toward Reliable Density Functional Methods without Adjustable Parameters: The PBE0 Model. J. Chem. Phys. 1999, 110, 6158-6170.

(10) Grimme, S.; Antony, J.; Ehrlich, S.; Krieg, H. A Consistent and Accurate Ab Initio Parametrization of Density Functional Dispersion Correction (DFT-D) for the 94 Elements H-Pu. J. Chem. Phys. 2010, 132, 154104.

(11) Grimme, S.; Ehrlich, S.; Goerigk, L. Effect of the Damping Function in Dispersion Corrected Density Functional Theory. J. Comput. Chem. 2011, 32, 1456-1465.

(12) Schäfer, A.; Horn, H.; Ahlrichs, R. Fully Optimized Contracted Gaussian Basis Sets for Atoms Li to Kr. J. Chem. Phys. 1992, 97, 2571-2577.

(13) Andrae, D.; Häußermann, U.; Dolg, M.; Stoll, H.; Preuß, H. Energy-Adjusted Ab Initio Pseudopotentials for the Second and Third Row Transition Elements. Theor. Chim. Acta 1990, 77, 123141.

(14) Peterson, K. A. Systematically Convergent Basis Sets with Relativistic Pseudopotentials. I. Correlation Consistent Basis Sets for the Post-d Group 13-15 Elements. J. Chem. Phys. 2003, 119, 1109911112.

(15) Marenich, A. V.; Cramer, C. J.; Truhlar, D. G. Universal Solvation Model Based on Solute Electron Density and on a Continuum Model of the Solvent Defined by the Bulk Dielectric Constant and Atomic Surface Tensions. J. Phys. Chem. B 2009, 113, 6378-6396.

(16) Weigend, F.; Ahlrichs, R. Balanced Basis Sets of Split Valence, Triple Zeta Valence and Quadruple Zeta Valence Quality for H to Rn: Design and Assessment of Accuracy. Phys. Chem. Chem. Phys. 2005, 7, 3297.

(17) Reich, H. J. Role of Organolithium Aggregates and Mixed Aggregates in Organolithium Mechanisms. Chem. Rev. 2013, 113, 7130-7178. 